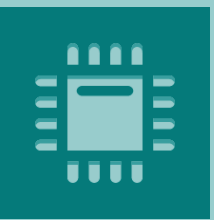

\title{
Sensors for Fluid Leak Detection
}

Edited by

Gonzalo Pajares Martinsanz

Printed Edition of the Special Issue Published in Sensors 
Gonzalo Pajares Martinsanz (Ed.)

\section{Sensors for Fluid Leak Detection}


This book is a reprint of the special issue that appeared in the online open access journal Sensors (ISSN 1424-8220) in 2014 (available at:

http://www.mdpi.com/journal/sensors/special_issues/leak-detection).

\section{Guest Editor}

Gonzalo Pajares Martinsanz

Department Software Engineering and Artificial Intelligence

Faculty of Informatics, University Complutense of Madrid

28040 Madrid

Spain

Editorial Office

MDPI AG

Klybeckstrasse 64

Basel, Switzerland

Publisher

Shu-Kun Lin

Production Editor

Lin $\mathrm{Li}$

\section{Edition 2015}

MDPI • Basel • Beijing • Wuhan

ISBN 978-3-03842-068-2 (Hbk)

ISBN 978-3-03842-069-9 (PDF)

(C) 2015 by the authors; licensee MDPI, Basel, Switzerland. All articles in this volume are Open Access distributed under the Creative Commons License (CC BY), which allows users to download, copy and build upon published articles even for commercial purposes, as long as the author and publisher are properly credited, which ensures maximum dissemination and a wider impact of our publications. However, the dissemination and distribution of physical copies of this book as a whole is restricted to MDPI, Basel, Switzerland. 


\section{Table of Contents}

List of Contributors VI

About the Guest Editor $\mathrm{X}$

\section{Gonzalo Pajares Martinsanz}

Editorial: Sensors for Fluid Leak Detection

Reprinted from: Sensors 2015, 15(2), 3830-3833

http://www.mdpi.com/1424-8220/15/2/3830

\section{Patricia Martínez-Garzón, Marco Bohnhoff, Grzegorz Kwiatek,}

\section{Gonzalo Zambrano-Narváez and Rick Chalaturnyk}

Microseismic Monitoring of $\mathrm{CO}_{2}$ Injection at the Penn West Enhanced Oil Recovery Pilot Project, Canada: Implications for Detection of Wellbore Leakage

Reprinted from: Sensors 2013, 13(9), 11522-11538

http://www.mdpi.com/1424-8220/13/9/11522

Aitor Ibarguren, Jorge Molina, Loreto Susperregi and Iñaki Maurtua

Thermal Tracking in Mobile Robots for Leak Inspection Activities

Reprinted from: Sensors 2013, 13(10), 13560-13574

http://www.mdpi.com/1424-8220/13/10/13560

\section{Myrna V. Casillas, Vicenç Puig, Luis E. Garza-Castañón and Albert Rosich}

Optimal Sensor Placement for Leak Location in Water Distribution Networks Using Genetic

Algorithms

Reprinted from: Sensors 2013, 13(11), 14984-15005

http://www.mdpi.com/1424-8220/13/11/14984

David Ayala-Cabrera, Manuel Herrera, Joaquín Izquierdo, Silvia J. Ocaña-Levario and Rafael Pérez-García

GPR-Based Water Leak Models in Water Distribution Systems

Reprinted from: Sensors 2013, 13(12), 15912-15936

http://www.mdpi.com/1424-8220/13/12/15912 
Abdulaziz S. Almazyad, Yasser M. Seddiq, Ahmed M. Alotaibi, Ahmed Y. Al-Nasheri, Mohammed S. BenSaleh, Abdulfattah M. Obeid and Syed Manzoor Qasim

A Proposed Scalable Design and Simulation of Wireless Sensor Network-Based LongDistance Water Pipeline Leakage Monitoring System

Reprinted from: Sensors 2014, 14(2), 3557-3577

http://www.mdpi.com/1424-8220/14/2/3557 80

Adnan Nasir, Syed Imtiaz Hussain, Boon-Hee Soong and Khalid Qaraqe

Energy Efficient Cooperation in Underlay RFID Cognitive Networks for a Water Smart Home

Reprinted from: Sensors 2014, 14(10), 18353-18369

http://www.mdpi.com/1424-8220/14/10/18353 101

Jeffrey Tuck and Pedro Lee

Inverse Transient Analysis for Classification of Wall Thickness Variations in Pipelines

Reprinted from: Sensors 2013, 13(12), 17057-17066

http://www.mdpi.com/1424-8220/13/12/17057

Fabrício Almeida, Michael Brennan, Phillip Joseph, Stuart Whitfield, Simon Dray and Amarildo Paschoalini

On the Acoustic Filtering of the Pipe and Sensor in a Buried Plastic Water Pipe and its Effect on Leak Detection: An Experimental Investigation

Reprinted from: Sensors 2014, 14(3), 5595-5610

http://www.mdpi.com/1424-8220/14/3/5595

Tung-Ching Su and Ming-Der Yang

Application of Morphological Segmentation to Leaking Defect Detection in Sewer Pipelines Reprinted from: Sensors 2014, 14(5), 8686-8704

http://www.mdpi.com/1424-8220/14/5/8686

Richard Bates, Michele Battistin, Stephane Berry, Alexander Bitadze, Pierre Bonneau, Nicolas Bousson, George Boyd, Gennaro Bozza, Olivier Crespo-Lopez, Enrico Da Riva, Cyril Degeorge, Cecile Deterre, Beniamino DiGirolamo, Martin Doubek, Gilles Favre, Jan Godlewski, Gregory Hallewell, Ahmed Hasib, Sergey Katunin, Nicolas Langevin, Didier Lombard, Michel Mathieu, Stephen McMahon, Koichi Nagai, Benjamin Pearson, David Robinson, Cecilia Rossi, Alexandre Rozanov, Michael Strauss, Michal Vitek, Vaclav Vacek and Lukasz Zwalinski

Implementation of Ultrasonic Sensing for High Resolution Measurement of Binary Gas Mixture Fractions

Reprinted from: Sensors 2014, 14(6), 11260-11276

http://www.mdpi.com/1424-8220/14/6/11260 
Pejman Razi and Farid Taheri

A Vibration-Based Strategy for Health Monitoring of Offshore Pipelines' Girth-Welds Reprinted from: Sensors 2014, 14(9), 17174-17191

http://www.mdpi.com/1424-8220/14/9/17174

Bing-Yu Wang, Fan-Chun Hsieh, Che-Yu Lin, Shao-En Chen, Fong-Zhi Chen and Chia-Che Wu

Development of a Piezoelectric Vacuum Sensing Component for a Wide Pressure Range

Reprinted from: Sensors 2014, 14(11), 22099-22112

http://www.mdpi.com/1424-8220/14/11/22099 


\section{List of Contributors}

Abdulaziz S. Almazyad: Department of Computer Engineering, King Saud University, Riyadh 11421, Saudi Arabia

Fabrício Almeida: Department of Mechanical Engineering, UNESP - FEIS, Av. Brasil, 56, 15385000, Ilha Solteira, Brazil

Ahmed Y. Al-Nasheri: Department of Computer Engineering, King Saud University, Riyadh 11421, Saudi Arabia

Ahmed M. Alotaibi: Department of Computer Engineering, King Saud University, Riyadh 11421, Saudi Arabia

David Ayala-Cabrera: FluIng-IMM, Universitat Politècnica de València, Camino de Vera s/n Edif. 5C 46022, Valencia, Spain

Richard Bates: SUPA School of Physics and Astronomy, University of Glasgow, G627QB, UK

Michele Battistin: CERN, 1211 Geneva 23, Switzerland

Mohammed S. BenSaleh: National Center for Electronics, Communications and Photonics Research, King Abdulaziz City for Science and Technology (KACST), Riyadh 11442, Saudi Arabia

Stephane Berry: CERN, 1211 Geneva 23, Switzerland

Alexander Bitadze: SUPA School of Physics and Astronomy, University of Glasgow, G627QB, UK

Marco Bohnhoff: Helmholtz Centre Potsdam GFZ German Research Centre for Geosciences, Section

3.2 Geomechanics and Rheology, Telegrafenberg, Potsdam 14473, Germany; Institute of Geological

Sciences, Free University of Berlin, Berlin 14195, Germany

Pierre Bonneau: CERN, 1211 Geneva 23, Switzerland

Nicolas Bousson: CERN, 1211 Geneva 23, Switzerland

George Boyd: Department of Physics \& Astronomy, University of Oklahoma, Norman, OK 73019, USA

Gennaro Bozza: CERN, 1211 Geneva 23, Switzerland

Michael Brennan: Department of Mechanical Engineering, UNESP - FEIS, Av. Brasil, 56, 15385000, Ilha Solteira, Brazil

Myrna V. Casillas: Supervision and Advanced Control Chair, Tecnológico de Monterrey, Campus Monterrey, Av. Eugenio Garza Sada 2501, Monterrey 64849, Mexico

Rick Chalaturnyk: Department of Civil and Environmental Engineering, University of Alberta, Edmonton, AB T6G 2W2, Canada

Fong-Zhi Chen: Instrument Technology Research Center, National Applied Research Laboratories, 20, R\&D Rd. VI, Hsinchu Science Park, Hsinchu 300, Taiwan

Shao-En Chen: Department of Mechanical Engineering, National Chung Hsing University, 250, Taichung 402, Taiwan

Olivier Crespo-Lopez: CERN, 1211 Geneva 23, Switzerland

Cyril Degeorge: Department of Physics, Indiana University, Bloomington, IN 47405 7015, USA

Cecile Deterre: Deutsches Elektronen-Synchrotron, Notkestraße 85, D-22607 Hamburg, Germany

Beniamino DiGirolamo: CERN, 1211 Geneva 23, Switzerland 
Martin Doubek: Czech Technical University, Technická 4, 16607, Praha Czech Republic

Simon Dray: Hydrosave Ltd, Swallow Court, Venture Park, Kettering NN15 6XX, UK

Gilles Favre: CERN, 1211 Geneva 23, Switzerland

Luis E. Garza-Castañón: Supervision and Advanced Control Chair, Tecnológico de Monterrey, Campus Monterrey, Av. Eugenio Garza Sada 2501, Monterrey 64849, Mexico

Jan Godlewski: CERN, 1211 Geneva 23, Switzerland

Gregory Hallewell: Centre de Physique des Particules de Marseille, 163 Avenue de Luminy, 13288 Marseille, France

Ahmed Hasib: Department of Physics \& Astronomy, University of Oklahoma, Norman, OK 73019, USA

Manuel Herrera: BaTir, Université libre de Bruxelles, Av. F. Roosvelt 50 B-1050, Brussels, Belgium

Fan-Chun Hsieh: Instrument Technology Research Center, National Applied Research Laboratories, 20, R\&D Rd. VI, Hsinchu Science Park, Hsinchu 300, Taiwan

Syed Imtiaz Hussain: Electrical and Computer Engineering Department, Texas A\&M University at Qatar, Doha 23874, Qatar

Aitor Ibarguren: IK4-Tekniker, Calle Iñaki Goenaga 5, Eibar 20600, Spain

Joaquín Izquierdo: FluIng-IMM, Universitat Politècnica de València, Camino de Vera s/n Edif. 5C 46022, Valencia, Spain

Phillip Joseph: Institute of Sound and Vibration Research-ISVR, University Road, SO17 1BJ, Southampton, UK

Sergey Katunin: Konstantinov Petersburg Nuclear Physics Institute (PNPI), Orlova Roscha, RU-188300, Russia

Grzegorz Kwiatek: Helmholtz Centre Potsdam GFZ German Research Centre for Geosciences, Section 3.2 Geomechanics and Rheology, Telegrafenberg, Potsdam 14473, Germany Nicolas Langevin: Centre de Physique des Particules de Marseille, 163 Avenue de Luminy, 13288 Marseille, France

Pedro Lee: Department of Civil and Natural Resources Engineering, University of Canterbury, Private Bag 4800, Christchurch 8041, New Zealand

Che-Yu Lin: Department of Mechanical Engineering, National Chung Hsing University, 250, Taichung 402, Taiwan

Didier Lombard: CERN, 1211 Geneva 23, Switzerland

Patricia Martínez-Garzón: Helmholtz Centre Potsdam GFZ German Research Centre for Geosciences, Section 3.2 Geomechanics and Rheology, Telegrafenberg, Potsdam 14473, Germany

Gonzalo Pajares Martinsanz: Department of Software Engineering and Artificial Intelligence, Faculty of Informatics, University Complutense of Madrid, 28040 Madrid, Spain

Michel Mathieu: Centre de Physique des Particules de Marseille, 163 Avenue de Luminy, 13288 Marseille, France

Iñaki Maurtua: IK4-Tekniker, Calle Iñaki Goenaga 5, Eibar 20600, Spain

Stephen McMahon: STFC Rutherford Appelton Laboratory-Harwell Oxford, Didcot, OX11 OQX, UK

Jorge Molina: IK4-Tekniker, Calle Iñaki Goenaga 5, Eibar 20600, Spain

Koichi Nagai: Department of Physics, Oxford University, Oxford OX1 3RH, UK 
Adnan Nasir: Electrical and Computer Engineering Department, Texas A\&M University at Qatar, Doha 23874, Qatar

Abdulfattah M. Obeid: National Center for Electronics, Communications and Photonics Research, King Abdulaziz City for Science and Technology (KACST), Riyadh 11442, Saudi Arabia

Silvia J. Ocaña-Levario: FluIng-IMM, Universitat Politècnica de València, Camino de Vera s/n Edif. 5C 46022, Valencia, Spain

Amarildo Paschoalini: Department of Mechanical Engineering, UNESP - FEIS, Av. Brasil, 56, 15385-000, Ilha Solteira, Brazil

Benjamin Pearson: Department of Physics \& Astronomy, University of Oklahoma, Norman, OK 73019, USA

Rafael Pérez-García: FluIng-IMM, Universitat Politècnica de València, Camino de Vera s/n Edif. 5C 46022, Valencia, Spain

Vicenç Puig: SAC Research Group, Institut de Robótica i Informàtica Industrial (IRI-CSIC), Universitat Politècnica de Catalunya (UPC), Llorens i Artigues, 4-6, Barcelona 08028, Spain

Khalid Qaraqe: Electrical and Computer Engineering Department, Texas A\&M University at Qatar, Doha 23874, Qatar

Syed Manzoor Qasim: National Center for Electronics, Communications and Photonics Research, King Abdulaziz City for Science and Technology (KACST), Riyadh 11442, Saudi Arabia

Pejman Razi: Advanced Composites and Mechanics Laboratory, Department of Civil and Resource Engineering, Dalhousie University, 1360 Barrington Street, Halifax, NS, B3H 4R2, Canada

Enrico Da Riva: CERN, 1211 Geneva 23, Switzerland

David Robinson: Department of Physics and Astronomy, University of Cambridge, Cambridge, CB3 0HE, UK

Albert Rosich: Interdisciplinary Centre for Security, Reliability and Trust, University of Luxembourg, 4, rue Alphonse Weicker L-2721, Luxembourg

Cecilia Rossi: Academy of Sciences of the Czech Republic, 11000 Praha, Czech Republic

Alexandre Rozanov: Centre de Physique des Particules de Marseille, 163 Avenue de Luminy, 13288 Marseille, France

Yasser M. Seddiq: Department of Computer Engineering, King Saud University, Riyadh 11421, Saudi Arabia; National Center for Electronics, Communications and Photonics Research, King Abdulaziz City for Science and Technology (KACST), Riyadh 11442, Saudi Arabia

Boon-Hee Soong: School of Electrical and Electronic Engineering, Nanyang Technological University, 639798, Singapore

Michael Strauss: Department of Physics \& Astronomy, University of Oklahoma, Norman, OK 73019, USA

Tung-Ching Su: Department of Civil Engineering and Engineering Management, National Quemoy University, Da Xue Rd. 1, Kinmen 892, Taiwan

Loreto Susperregi: IK4-Tekniker, Calle Iñaki Goenaga 5, Eibar 20600, Spain

Farid Taheri: Advanced Composites and Mechanics Laboratory, Department of Civil and Resource Engineering, Dalhousie University, 1360 Barrington Street, Halifax, NS, B3H 4R2, Canada

Jeffrey Tuck: Department of Civil and Natural Resources Engineering, University of Canterbury, Private Bag 4800, Christchurch 8041, New Zealand 
Vaclav Vacek: Czech Technical University, Technická 4, 16607, Praha Czech Republic

Michal Vitek: Czech Technical University, Technická 4, 16607, Praha Czech Republic

Bing-Yu Wang: Department of Mechanical Engineering, National Chung Hsing University, 250, Taichung 402, Taiwan

Stuart Whitfield: South Staffs Water plc, Green Lane, Walsall WS2 7PD, UK

Chia-Che Wu: Department of Mechanical Engineering, National Chung Hsing University, 250, Taichung 402, Taiwan

Ming-Der Yang: Department of Civil Engineering, National Chung Hsing University, Taichung 402, Taiwan

Gonzalo Zambrano-Narváez: Department of Civil and Environmental Engineering, University of Alberta, Edmonton, AB T6G 2W2, Canada

Lukasz Zwalinski: CERN, 1211 Geneva 23, Switzerland 


\section{About the Guest Editor}

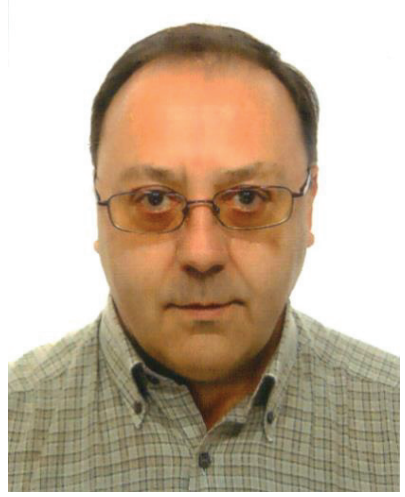

Gonzalo Pajares Martinsanz is a Professor at University Complutense of Madrid (UCM), Madrid, Spain. He received the Ph.D. degree in physics in 1995, from UNED, Madrid, Spain, discussing a thesis on the application of pattern recognition techniques to stereovision. Since 1990, he worked in Indra in critical real-time software development. He was also working in Indra Space and for the Spanish National Institute for Aerospace Technology developing remote sensing applications based on advanced image processing and computer vision techniques. He joined the UCM in 1995, as an Associate Professor and from 2004 as a FullTime Professor in computer science in the Department of Software Engineering and Artificial Intelligence. He is co-director of the ISCAR Research Group whose focus is on robotics, automatic control, and artificial intelligence. His research interests are computer vision, image understanding and processing, pattern recognition, and decisionmaking. He has authored and co-authored more than 150 publications, including 80 papers indexed on the journal citation report (JCR) papers, in such areas. He is the first Editor-in-Chief of the Journal of Imaging. He also serves as an Editorial Board member in the following SCI-JCR ranked journals: Sensors (Physical Sensors), Remote Sensing, EURASIP Journal on Image and Video Processing and Pattern Analysis and Applications. He has been the principal researcher with more than 20 international and national research projects with technological transference to the industry. He is an IEEE Senior Member and has participated as a Program Committee member in more than 35 international events and Guest Editor in Sensors and Remote Sensing journals with about 300 papers published. 


\section{Editorial}

\section{Sensors for Fluid Leak Detection}

\section{Gonzalo Pajares Martinsanz}

Department of Software Engineering and Artificial Intelligence, Faculty of Informatics, University Complutense of Madrid, 28040 Madrid, Spain; E-Mail: pajares@ucm.es; Tel.: +34-1-394-7546; Fax: +34-1-394-7547

Academic Editor: Vittorio M.N. Passaro

Received: 1 February 2015 / Accepted: 5 February 2015 / Published: 5 February 2015

Fluid leak detection represents a problem that has attracted the interest of researchers, but not exclusively because in industries and services leaks are frequently common. Indeed, in water or gas supplies, chemical or thermal plants, sea-lines or cooling/heating systems leakage rates can cause important economic losses and sometimes, what it is more relevant, environmental pollution with human, animal or plant lives at risk. This last issue has led to increased national and international regulations with different degrees of severity regarding environmental conservation.

Early fluid detection represents an important challenge to avoid the problems mentioned above. This special issue was proposed with the aim of attracting new technological developments and methods in sensors based on physical, chemical or biological principles. The following is a summary of works published in this special issue representing the achievements obtained. They are organized according to the main topics and applications addressed in the special issue, which can serve to the reader as a first introduction guide to each work:

1. $\mathrm{CO}_{2}$ and $\mathrm{CH}_{4}$ leakage in natural reservoir with micro-seismicity: In [1] an array of eight short-period borehole geophones, three pressure-temperature sensors and two fluid-sample sensors were deployed with the aim of detecting induced micro-seismicity associated with $\mathrm{CO}_{2}$ activity, including injection, within a natural reservoir in the Pembina oil field in Alberta/Canada. The primary objective was to investigate potentially occurring $\mathrm{CO}_{2}$-induced seismic signatures on a two-week period framing a substantial $\mathrm{CO}_{2}$ and $\mathrm{CH}_{4}$ leakage.

2. Thermal power plants: An autonomous robotic system is designed in [2] to perform pipeline inspection for early detection and prevention of leakages in solar thermal power plants. A thermographic camera provides the required information to detect leakages in collectors and pipelines and also for tracking purposes. Experiments were conducted in solar plants at the Torresol Energy Investments S.A. facilities in San José del Valle, Spain. The solar field is composed of nearly 7500 parabolic cylinder collectors that transport a Heat Transfer Fluid (HFT), which absorbs the solar energy. HFT circulates at high temperature $\left(\sim 390{ }^{\circ} \mathrm{C}\right)$ inside the absorber tubes. HTF leakages detection is the final goal of the proposed system. 
3. Water distribution systems: Sensors, technologies, methods or procedures can be developed to reduce the amount of water leaked in distribution systems. An optimum sensor deployment, based on genetic algorithms, is proposed in [3] for leak detection, isolation and location in water distribution networks. The proposed approach was tested on the water network of Hanoi, Vietnam with 31 demand nodes, one reservoir node and 34 pipes. In [4] a Ground Penetrating Radar is the technology used for leaks detection in water distribution systems, the instrument is equipped with a monostatic antenna operating at a central frequency of $1.5 \mathrm{GHz}$. The data collected as images are conveniently processed and analyzed based on the identification of vertical and horizontal profiles in the images. Wireless Sensor Networks and Radio Frequency IDentification (RFID) is the technology used in [5] for the design and simulation of a water pipeline leakage monitoring system. The design is based on deploying a group of mobile wireless sensor nodes and allowing them to work cooperatively according to a prescheduled order. Only a node is active at a time while the remainders are sleeping, which are activated based on three kinds of events: location-based, time-based and interrupt-based. Each node, equipped with a pressure sensor, a microcontroller and a RFID reader, records pressures and its location based on its exposure to signals of active RFID tags placed outside of the pipeline surface. The mobile sensor nodes move with the water current from the pipeline source down to the sink where the node is collected and its memory content transferred to a computer for numerical analysis. In the context of water distribution systems at home, numerical models are tested in [6] to determine when an event occurs (tap open, high/low water consumption, seepage). This includes leak detection. The hierarchical hidden Markov model (HHMM) is the method used for the recognition of such events. RFIDs integrated with pressure sensors are embedded in the pipe infrastructure. They collect pressure information and send it along with their IDs to the reader/writer destination node to determine the pattern of the event. The network infrastructure, together with the communication system, configures a water smart home system that allows monitoring the water distribution system and indirectly the leak detection.

4. Thinning of pipe walls: Leaks can be caused by degradation in the pipelines' walls, sometimes expressed as a thinning. In this regard, in [7] the analysis of transient fluid pressure signals has been studied and analyzed for detecting thinning in pipelines. This is carried out by placing high speed pressure sensors in contact with the fluid. Analysis based on numerical models is applied to detect the transient flow variations inside the pipeline where the geometry changes because of the thinning.

5. Buried plastic pipes: Experimental studies have been conducted in [8] in order to validate leak detection methods in buried plastic pipes using measurements of acoustic pressures (captured with hydrophones), velocities (using geophones), and accelerations (obtained with accelerometers). The leaks generate broadband noise, which propagates along the pipe, both in the fluid and along the pipe-wall either side of the leak, to sensors conveniently located at access points. Because in water plastic pipes the pipe-wall and water exhibit strong acoustic coupling measurements of leak noises can be made in the pipe for leak detection. The differences in the arrival times of the noise at the sensors' locations (time delays) determine the position of the leak. 
6. Sewer pipelines: Closed Circuit Television (CCTV) is the technology used in [9] for detecting leaks in vitrified clay sewer pipes, where the failure level and the position are to be determined. Computer vision techniques based on edge detection and morphology image segmentation operations are used for such purpose. The CCTV was mounted on a robot connected on the ground with a power cable and the Taichung City in Taiwan inspected with the robot traveling between manholes.

7. Ultrasonic in binary gas: In [10] an ultrasonic instrument is used to measurement of leaks of a high molecular weight gas (octafluoropropane coolant, $\mathrm{C}_{3} \mathrm{~F}_{8}$ ) into a system that is nominally composed of a single gas (nitrogen) during a long duration (18 month). The sensitivity of the instrument is due to the difference in molecular masses of the two gases in the mixture. The impact of variables such as temperature and pressure on the accuracy of the measurement is analyzed. Ultrasonic bursts are propagated in a sealed tube designed to provide a smooth flowing gas region between two transducers. Each transducer is comprised of a thin, Au plated foil stretched over a spirally grooved conductive disk. The foil is held at ground potential and the disk is biased between $100 \mathrm{Vdc}$ and $360 \mathrm{Vdc}$. A $50 \mathrm{kHz}$ pulse train modulates the transducer bias voltage, exciting the diaphragm to transmit an acoustic wave in response to the fluctuating electric field. A micro-controller creates gated pulses and measures the time of fight pulses between the transmitter and receiver transducers.

8. Offshore Pipelines: Girth-welds in offshore submerged pipelines has been numerically analyzed in [11] because their potential to cause leaks. Eight piezoelectric transducers (sensors/actuators), enclosed by a special waterproof coating, were bonded to an aluminum pipe in either sides of the girth-welds. The electrical connections were sealed manually with silicon and secured by heat shrink sleeves. Two excitation methods were applied for conducting the damage detection trials during the experiments: (a) impact, with a waterproofed pneumatic hammer; (b) chirp waves ranging in 10-5000 Hz, produced by using one of the piezoelectric transducers as actuator and conveniently amplified.

9. Pressure sensors: Pressure changes in fluid distribution networks may be evidences of malfunctioning and perhaps of leaks. A clamped-clamped beam-type piezoelectric vacuum pressure sensing device is designed in [12]. The piezoelectric element together with a self-actuating and a self-sensing microresonator detect the damping ratio of the gas, which allows enabling the calculation of the pressure of the vacuum system. The sensing element comprises a piezoelectric ceramic lead zirconate titanate (PZT) layer, a substrate and two pairs of electrodes. A pair is used to apply a sinusoidal voltage signal and the second pair receives the vibrations. The received vibrations are finally converted to electric energy using the positive piezoelectric effect. The output voltages, which varied under different gases viscosity and vacuum pressures, are measured by the device. 


\section{Acknowledgments}

Thanks are due to all the authors for their valuable collaboration and contributions to this special issue, as without them it could not exist. All papers presented to the call passed a rigorous refereeing process as full manuscripts. The accepted papers underwent final revision and approval for publication in a second/third round of reviewing. Gratitude is owed to the international team of reviewers for their diligence in assessing the papers and their thoughtful and constructive criticism with a great effort and dedication of their time. Extensive compilation of sensory technologies included in this special issue represents a significant contribution to research in the future.

\section{Conflicts of Interest}

The author declares no conflict of interest

Gonzalo Pajares Martinsanz

Guest Editor

\section{References}

1. Martínez-Garzón, P.; Bohnhoff, M.; Kwiatek, G.; Zambrano-Narváez, G.; Chalaturnyk, R. Microseismic Monitoring of CO2 Injection at the Penn West Enhanced Oil Recovery Pilot Project, Canada: Implications for Detection of Wellbore Leakage. Sensors 2013, 13, 11522-11538.

2. Ibarguren, A.; Molina, J.; Susperregi, L.; Maurtua, I. Thermal Tracking in Mobile Robots for Leak Inspection Activities. Sensors 2013, 13, 13560-13574.

3. Casillas, M.V.; Puig, V.; Garza-Castañón, L.E.; Rosich, A. Optimal Sensor Placement for Leak Location in Water Distribution Networks Using Genetic Algorithms. Sensors 2013, 13, 14984-15005.

4. Ayala-Cabrera, D.; Herrera, M.; Izquierdo, J.; Ocaña-Levario, S.J.; Pérez-García, R. GPR-Based Water Leak Models in Water Distribution Systems. Sensors 2013, 13, 15912-15936.

5. Almazyad, A.S.; Seddiq, Y.M.; Alotaibi, A.M.; Al-Nasheri, A.Y.; BenSaleh, M.S.; Obeid, A.M.; Qasim, S.M. A Proposed Scalable Design and Simulation of Wireless Sensor Network-Based Long-Distance Water Pipeline Leakage Monitoring System. Sensors 2014, 14, 3557-3577.

6. Nasir, A.; Hussain, S.I.; Soong, B.-H.; Qaraqe, K. Energy Efficient Cooperation in Underlay RFID Cognitive Networks for a Water Smart Home. Sensors 2014, 14, 18353-18369.

7. Tuck, J.; Lee, P. Inverse Transient Analysis for Classification of Wall Thickness Variations in Pipelines. Sensors 2013, 13, 17057-17066.

8. Almeida, F.; Brennan, M.; Joseph, P.; Whitfield, S.; Dray, S.; Paschoalini, A. On the Acoustic Filtering of the Pipe and Sensor in a Buried Plastic Water Pipe and Its Effect on Leak Detection: An Experimental Investigation. Sensors 2014, 14, 5595-5610.

9. Su, T.-C.; Yang, M.-D. Application of Morphological Segmentation to Leaking Defect Detection in Sewer Pipelines. Sensors 2014, 14, 8686-8704. 
10. Bates, R.; Battistin, M.; Berry, S.; Bitadze, A.; Bonneau, P.; Bousson, N.; Boyd, G.; Bozza, G.; Crespo-Lopez, O.; Riva, E.D.; et al. Implementation of Ultrasonic Sensing for High Resolution Measurement of Binary Gas Mixture Fractions. Sensors 2014, 14, 11260-11276.

11. Razi, P.; Taheri, F. A Vibration-Based Strategy for Health Monitoring of Offshore Pipelines' Girth-Welds. Sensors 2014, 14, 17174-17191.

12. Wang, B.-Y.; Hsieh, F.-C.; Lin, C.-Y.; Chen, S.-E.; Chen, F.-Z.; Wu, C.-C. Development of a Piezoelectric Vacuum Sensing Component for a Wide Pressure Range. Sensors 2014, 14, 22099-22112. 



\title{
Microseismic Monitoring of $\mathrm{CO}_{2}$ Injection at the Penn West Enhanced Oil Recovery Pilot Project, Canada: Implications for Detection of Wellbore Leakage
}

\author{
Patricia Martínez-Garzón, Marco Bohnhoff, Grzegorz Kwiatek, \\ Gonzalo Zambrano-Narváez and Rick Chalaturnyk
}

\begin{abstract}
A passive seismic monitoring campaign was carried out in the frame of a $\mathrm{CO}_{2}$-Enhanced Oil Recovery (EOR) pilot project in Alberta, Canada. Our analysis focuses on a two-week period during which prominent downhole pressure fluctuations in the reservoir were accompanied by a leakage of $\mathrm{CO}_{2}$ and $\mathrm{CH}_{4}$ along the monitoring well equipped with an array of short-period borehole geophones. We applied state of the art seismological processing schemes to the continuous seismic waveform recordings. During the analyzed time period we did not find evidence of induced micro-seismicity associated with $\mathrm{CO}_{2}$ injection. Instead, we identified signals related to the leakage of $\mathrm{CO}_{2}$ and $\mathrm{CH}_{4}$, in that seven out of the eight geophones show a clearly elevated noise level framing the onset time of leakage along the monitoring well. Our results confirm that micro-seismic monitoring of reservoir treatment can contribute towards improved reservoir monitoring and leakage detection.
\end{abstract}

Reprinted from Sensors. Cite as: Martínez-Garzón, P.; Bohnhoff, M.; Kwiatek, G.; Zambrano-Narváez, G.; Chalaturnyk, R. Microseismic Monitoring of $\mathrm{CO}_{2}$ Injection at the Penn West Enhanced Oil Recovery Pilot Project, Canada: Implications for Detection of Wellbore Leakage. Sensors 2013, 13, 11522-11538.

\section{Introduction}

One of the key-challenges in the frame of long-term sequestration of $\mathrm{CO}_{2}$ is to deliver appropriate monitoring techniques to document and quantify the safe storage of $\mathrm{CO}_{2}$ at selected sites $[1,2]$. Amongst the approaches to monitor $\mathrm{CO}_{2}$ storage, Passive Seismic Monitoring (PSM) can deliver critical information on the effects of pressure perturbation and fracture generation [3,4]. PSM also allows tracing fluid propagation within the reservoir, caprock or along wellbores using locations of small-scale induced earthquakes detected at surface and/or borehole geophones [5].

PSM is a well-established method in both hydrocarbon and geothermal industries, where it is used to monitor reservoir stimulation as well as in fundamental research covering various applications in earthquake seismology. Several studies have used this technique to characterize the treatment of different types of reservoirs [6-12]. Despite the great potential of the method, it is still not systematically applied to the field of $\mathrm{CO}_{2}$ storage. However, recent discussions on the feasibility of large-scale $\mathrm{CO}_{2}$ storage include the potential risk posed by induced seismicity [13].

There is extensive knowledge supporting the idea that regions with the highest potential for $\mathrm{CO}_{2}$ storage are basins with thick sequences of sedimentary rocks [14]. This is the case at the Pembina oil field in Alberta/Canada, where the Cardium Formation (capping siltstones, shales, and sandstones) is confined between Marine Shales and the Blackstone Formation [15]. Recent studies support the view that injection in sedimentary rocks generally tends to be less seismogenic than in crystalline rocks [16]. This observation is consistent with sparse amounts of induced seismic events all being of low magnitude 
during and after $\mathrm{CO}_{2}$ injection in sedimentary formations $[17,18]$. However, in a recent $\mathrm{CO}_{2}$ storage site (In-Salah) many seismic events were induced [19]. In this sense, [20] have shown that the deformation and the geomechanical response of great $\mathrm{CO}_{2}$ storage fields can be very different from one site to another. This supports the idea that the few existing case studies cannot be used to generalize the potential for $\mathrm{CO}_{2}$ storage sites to generate seismic events. More pilot field studies are needed to derive quantitative statements on the probability of inducing micro-seismicity.

In 2005, the multidisciplinary research pilot project Penn West established by the Alberta Government started injecting supercritical $\mathrm{CO}_{2}$ to Enhance the Oil Recovery (EOR) at the Pembina Field [21,22]. At this site, the $\mathrm{CO}_{2}$ was injected into the Cardium Formation (1650 $\mathrm{m}$ depth) and a percentage of it was systematically released again dissolved in the produced oil. To monitor the $\mathrm{CO}_{2}$ injection, a PSM campaign was carried out between 2005 and 2008 using an array of eight three-component borehole geophones. Since the geophones are placed below the uppermost weathering layer and closer to the target reservoir, some of the advantages of using borehole geophones are the substantial improvements of noise conditions with respect to the surface as well as the reduction in the attenuation of the signals.

In this study, we analyse continuous seismic recordings framing a two-week period to investigate whether induced micro-seismicity occurred in the frame of the $\mathrm{CO}_{2}$ injection into the reservoir. The selected time period includes a substantial outflow of $\mathrm{CO}_{2}$ and $\mathrm{CH}_{4}$ (occurring on 1 September 2005 at 09:41 AM) observed at the well-head of the monitoring well where the sensors were deployed. Therefore, we also aim to further investigate whether the outflow resulted in any sort of seismic signatures at the borehole geophones that might serve for an improved detection of along-well gas flow (leakage). Different state-of-the-art seismological analysis methods to detect potential induced seismicity and/or elevated noise levels were performed.

\section{Data Acquisition}

To achieve a comprehensive multi-parameter monitoring of the target reservoir, instrumentation was deployed in a pre-existing vertical production well refurbished as monitoring well. The monitoring well was located at approximately $300 \mathrm{~m}$ lateral distance to the nearest injector well I1 (Figure 1a). 
Figure 1. (a) Map of the location of the Pembina oil field and location of the monitoring well (triangle) with respect to the injector and the producer wells. P.1-6: Producer wells. I.1, I.2: Injector wells (directional wells); (b) Lithological column and instrumentation deployed in the monitoring well. Geophone 1 (g.1) is the deepest sensor, placed at $1640 \mathrm{~m}$. Geophone 8 (g.8) is the shallowest, placed at $1,500 \mathrm{~m}$.

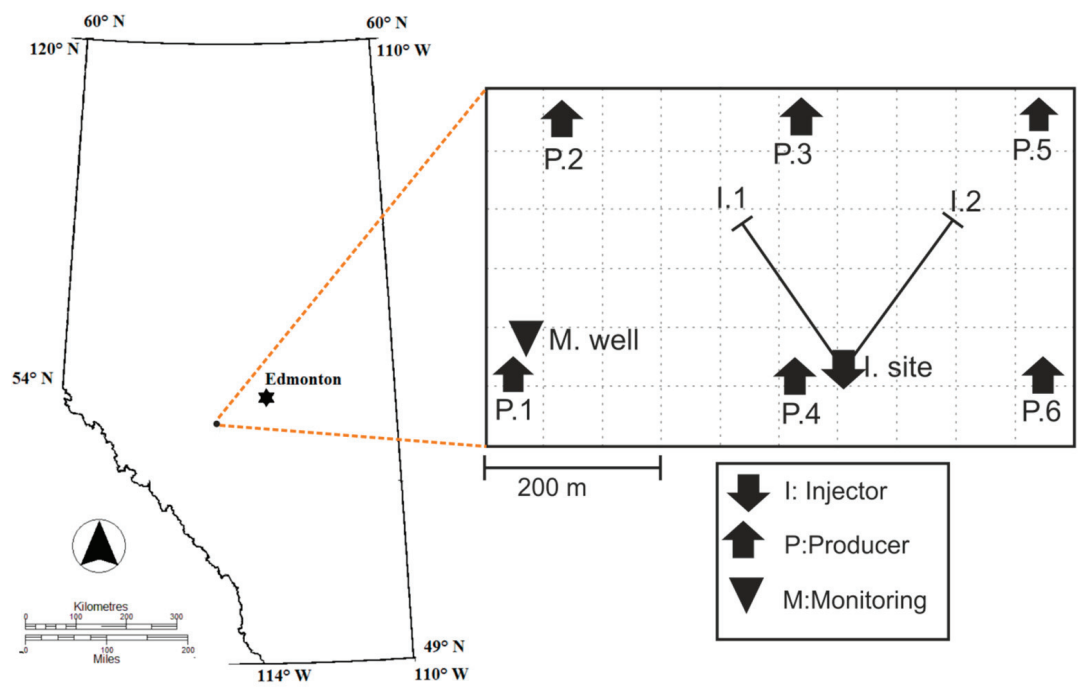

(a)

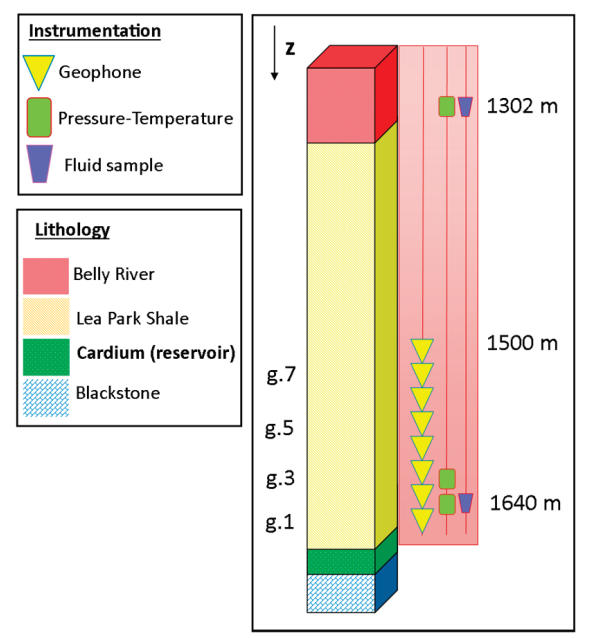

(b)

The deployed instrumentation at the monitoring well consisted of eight geophones, three pressure-temperature sensors and two fluid-sample sensors (Figure 1b). The instrumentation was attached to production tubing and placed inside the production casing. This procedure is common to reduce the installation damage. To improve the acoustic coupling of the sensors to the formation, cement was retained during the tubing string. However, cementing operations did not proceed as designed and a channel was created in the cement annulus [23]. This fact could affect the coupling of some of the sensors. 
The geophones, fabricated from 316 ELC stainless steel are three-component short-period sensors with a natural frequency of $24 \mathrm{~Hz}$ and nominal resistance of $12.8 \mathrm{k} \Omega$ per axis [24]. They were placed between 1,500 $\mathrm{m}$ and 1,640 $\mathrm{m}$ depth. Sampling frequency for continuous seismic recordings was set to $1 \mathrm{kHz}$. Theoretically, they allow to record signals up to $500 \mathrm{~Hz}$. Assuming a conservative average stress drop of $1 \mathrm{MPa}$, the sensor array should be able to detect nearby micro-seismicity with reasonable high signal-to-noise ratio for $\mathrm{M}_{\mathrm{w}}>-1.5$ [25]. This magnitude corresponds to seismic events with source radii of a few meters.

The data from the geophones was analogically acquired and transmitted to the surface. A maximum of four geophone housings could be linked together on a single, 24-conductor (12 pair) stranded copper electrical cable (one pair per each geophone component). Cables were jacketed for safety. This resulted in two electrical cables running to the surface. As the casing was lowered into the well, the geophones were still able to rotate around the vertical axis. For this reason, the horizontal orientation of each sensor is different [26].

\section{Methods}

We have applied different seismological techniques to the continuous seismic recordings to investigate the quality of the data, potential micro-seismic activity and $\mathrm{CO}_{2}$ leakage signatures. The applied methodologies are the following:

1. Spectrograms were generated to visually inspect the general frequency content of the waveform recordings. By using spectrograms, micro-seismic events can be identified by short-term amplitude increases in the higher frequency parts (usually $>100 \mathrm{~Hz}$, depending on magnitude and hypocentral distance). For this analysis, the waveforms were previously corrected for the baseline shift (detrended) and high-pass filtered $(0.8 \mathrm{~Hz})$ to remove potential long-period signals associated with seismic events not recordable by the used instrumentation. Additionally, the data displayed significant noise at $60 \mathrm{~Hz}$ and its multiples caused by electrical equipment located nearby. Signals at these frequencies were suppressed by applying two notch filters in the intervals 55-65 Hz and 115-125 Hz, respectively. We generated spectrograms for the entire analysed dataset by taking $1 \mathrm{~min}$ time-windows of vertical-component waveform data and calculating the short-time Fourier transform of the input signal.

2. We systematically analysed the average noise levels at each individual sensor to determine times of enhanced levels that might be associated with external processes such as e.g., nearby fluid flow. This analysis can also provide information as to the quality of the individual geophones (e.g., due to poor coupling or mechanical dysfunction). Here, we worked with only baseline-corrected data since we were interested in all the frequencies. The noise-level analysis for each individual geophone through the entire two-week data was based on one-minute long subsets.

3. We applied a signal-detection Short Time Average-Long Time Average (STA/LTA) [27] algorithm to identify micro-seismic signatures in the continuous waveform recordings. A STA/LTA trigger detects onset times of characteristic signals (e.g., seismic P and S waves) based on a pre-defined minimum ratio of average absolute amplitudes of two 
time windows with different length. The STA/LTA ratio will increase once an elastic wave reaches a geophone. When the threshold of the STA/LTA ratio is reached at a particular sensor, the time is saved. For this analysis, the data was processed as for the spectrogram calculation. First, the algorithm was appropriately tuned for this specific dataset. Then, we run the algorithm on the vertical components of the geophones over the entire analysed time period. Finally, a coincidence trigger was applied to the obtained geophone-specific detection lists to select only those seen at a minimum number of four geophones within a given time window $(40 \mathrm{~ms})$. To define the time window of the coincidence trigger, a homogeneous velocity model of $V_{P}=3.5 \mathrm{~km} / \mathrm{s}$ (slightly lower than the estimated $V_{P}$ for the formation in [26]) was used.

4. Lastly, we also looked for potential slow-slip processes included in the data. At reservoir scale, Long-Period and Long-Duration (LPLD) events were found in a multi-stage hydraulic fracturing experiment [28]. The authors described events observed during fracturing periods that have a typical duration of 10-100 s and most of their frequency content is in the $10-80 \mathrm{~Hz}$ interval. Recent studies [29] indicated that such events are not necessarily occurring in the frame of reservoir treatment involving hydraulic fracturing. We note that our project was designed to inject large amounts of fluids without causing hydraulic fractures in the target formation, and thus it was not very likely for such signals to occur. Nevertheless, such studies are still quite sparse and it is worth analysing the corresponding frequency band. Note that the frequencies lower than $24 \mathrm{~Hz}$ (the natural frequency of the sensors) will be diminished by the transfer function of the sensors. However, since LPLD are reported up to $80 \mathrm{~Hz}$, the available bandwidth to investigate is still sufficient to detect them if they occurred. For this analysis we applied a band-pass filter in the $5-100 \mathrm{~Hz}$ interval. We first stacked the amplitudes for all sensors (vertical components), and then calculated spectrograms of $50 \mathrm{~min}$ time-windows by stacking the spectral density of the vertical components.

\section{Results}

Before the described seismological analysis, manual review of the data revealed that for the last 1.5 days of the two-week period none of the channels of g.2, g.4 (partially) and g.6 were functioning. Additionally, it was also noticed that the horizontal components of these sensors display much lower amplitudes than the verticals during the entire analyzed period. Functionality of each geophone is summarized in Table 1. 
Table 1. Summary of geophone status obtained from spectrograms, noise level analysis and manual review of the data.

\begin{tabular}{ccc}
\hline Geophone ID & Depth $(\mathbf{m})$ & Geophone Functionality \\
\hline 8 & 1,500 & Correct \\
7 & 1,520 & Horizontal component 'x' not recording \\
6 & 1,540 & Horizontal components 'x' and 'y' not recording \\
5 & 1,560 & Correct \\
4 & 1,580 & Correct during certain periods \\
3 & 1,600 & Correct. High noise level (average amplitudes) \\
2 & 1,620 & Horizontal components 'x' and 'y' not recording \\
1 & 1,640 & Correct. High noise level (average amplitudes) \\
\hline
\end{tabular}

\subsection{Spectrograms}

The calculated spectrograms show that most of the energy in the recorded time series was transferred in the frequency interval up to $200 \mathrm{~Hz}$ (Figure 2). Interestingly, the spectrograms show several short time intervals of elevated energy up to $500 \mathrm{~Hz}$ (our Nyquist frequency). Such signals are part of the frequency characteristics of micro-seismic events and thus would need to be checked in detail. However, most of such signals generally do not show any temporal correlation between the individual geophones. This suggests that their origin cannot be external (e.g., related to the injected $\mathrm{CO}_{2}$ in the reservoir). For this reason, none of the clear high-amplitude signal seen at the sensors could be related to an induced micro-seismic event occurring off the array.

Figure 2. Vertical component waveform recordings and corresponding spectrograms calculated for each geophone framing twenty-minute time-windows around the onset time of the outflow (09:41). The amplitude of each frequency appears color-encoded. g.2 and g.7 have much lower energy recorded than the other geophones. g.1, g.3 and g.8 recorded many sharp spike-signals, although they do not occur at the same time. g.5 and g.6 show spikes with high amplitude, probably triggered internally. Additionally, they still display high electronic noise despite of the notch filter.
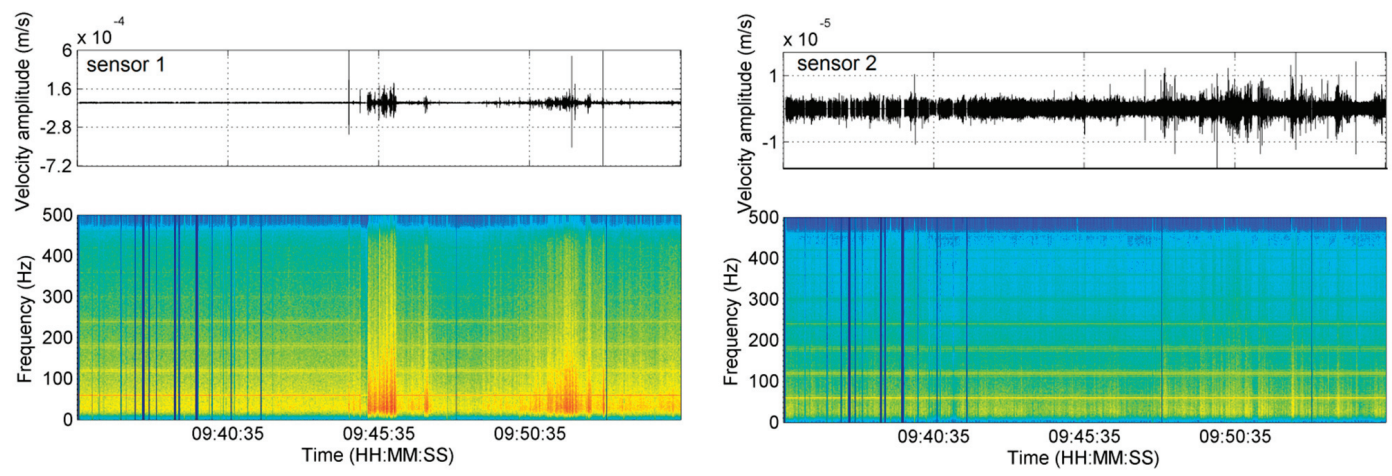
Figure 2. Cont.
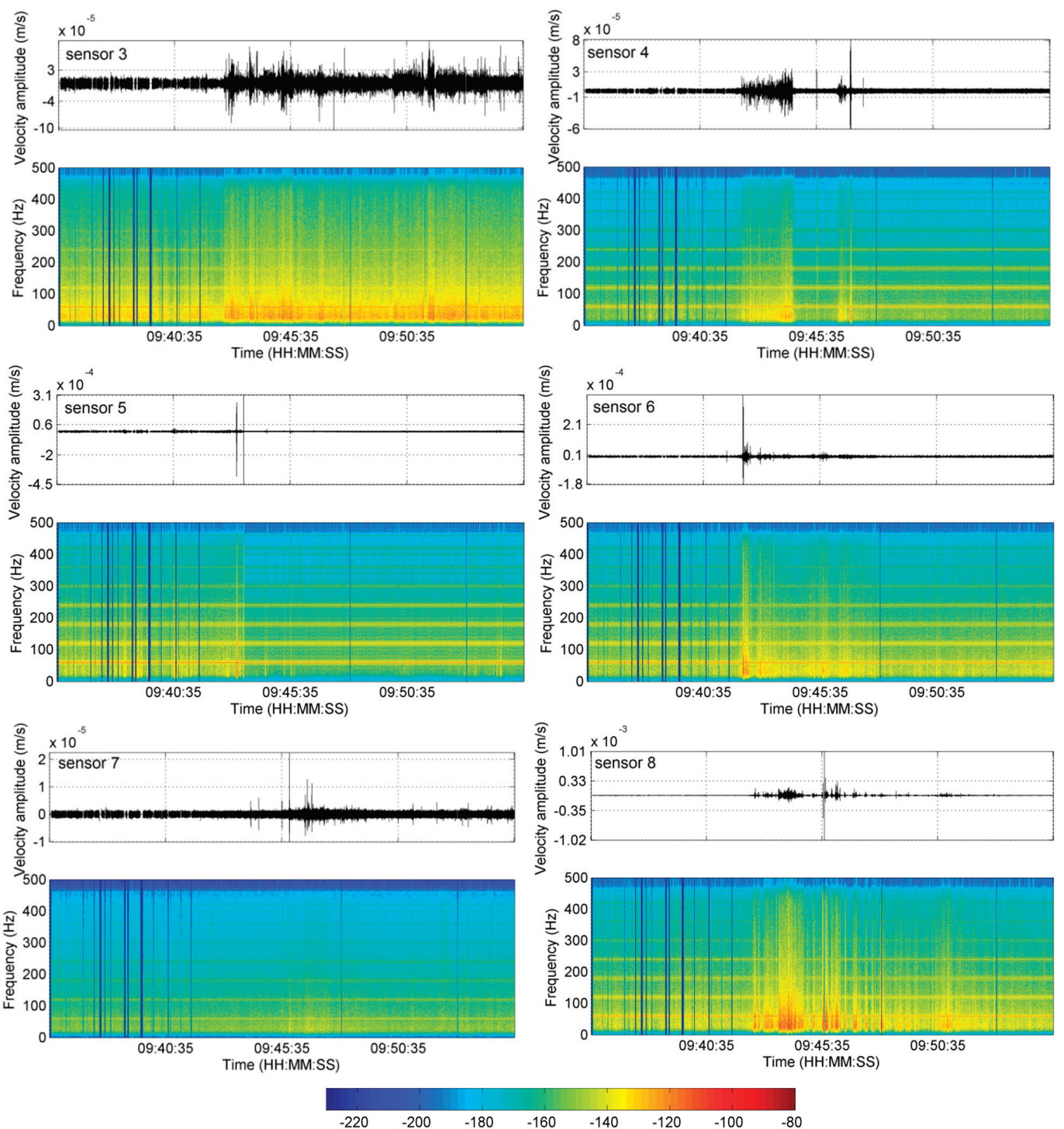

The spectrograms shown in Figure 2 cover a period of time framing the onset of the outflow (09:41). Many of the sensors show clear changes in the recorded frequency content before and after the onset of the outflow (Figure 2). After 09:41, much more energy is recorded. This energy is especially prominent up to approximately $120 \mathrm{~Hz}$.

Spectrograms were also used to investigate the quality of the coupling of the geophones to the tubing string. A general rule of thumb is, that the better the coupling, the larger is the bandwidth of the transfer function of a borehole geophone. In general, all eight geophones are capable to record also high frequencies indicating a reasonably good coupling to the well-casing (Figure 2). However, the deepest sensor (g.1) and also g.3 recorded overall higher energies. 


\subsection{Noise Analysis}

In general, noise amplitudes at the sensors g.1 and g.3 are higher than the noise levels at the other sensors. In addition, g. 2 and g. 6 recorded significantly lower amplitudes (on average three orders of magnitude less) than any other sensor. Comparing these observations and the manual data review with the field protocols, we found geophones with odd ID numbers shared one common cable and the geophones with even number shared a second one. This resulted in two cables running to the surface. Since common characteristics between the even geophones are found, a second explanation for the low amplitudes recorded would be a higher resistance of the cable resulting in higher attenuation of the signal.

Figure 3. (a) Four hours average noise levels at the sensors including the time of the outflow $(09: 41)$. Each trace is normalized to its overall maximum; (b) Twenty minutes vertical component waveform recordings framing the onset of the outflow. Each trace is normalized to the overall maximum. g: Geophone. Time of the outflow is indicated by the arrow.

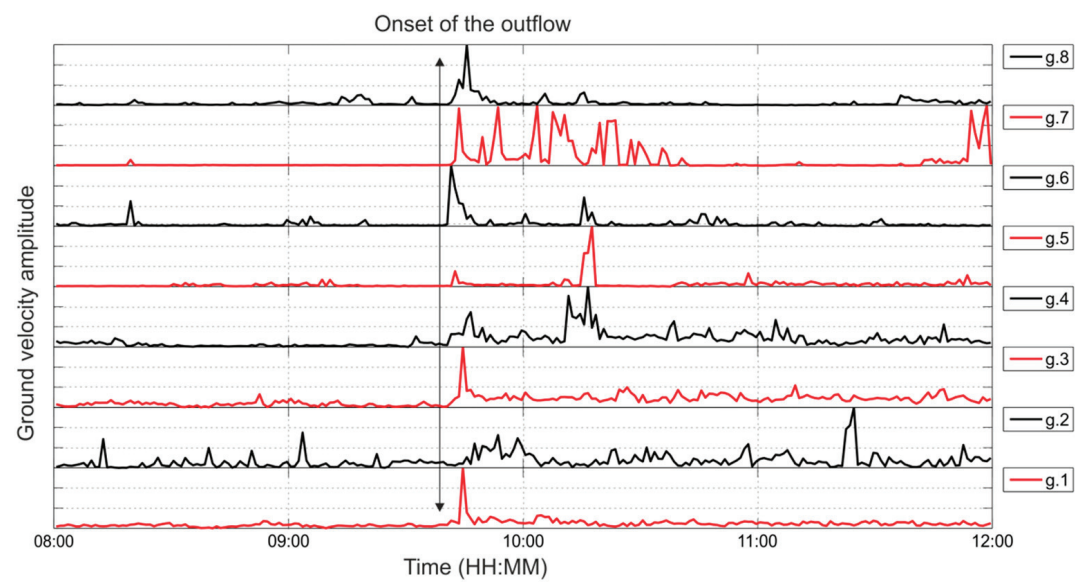

(a)

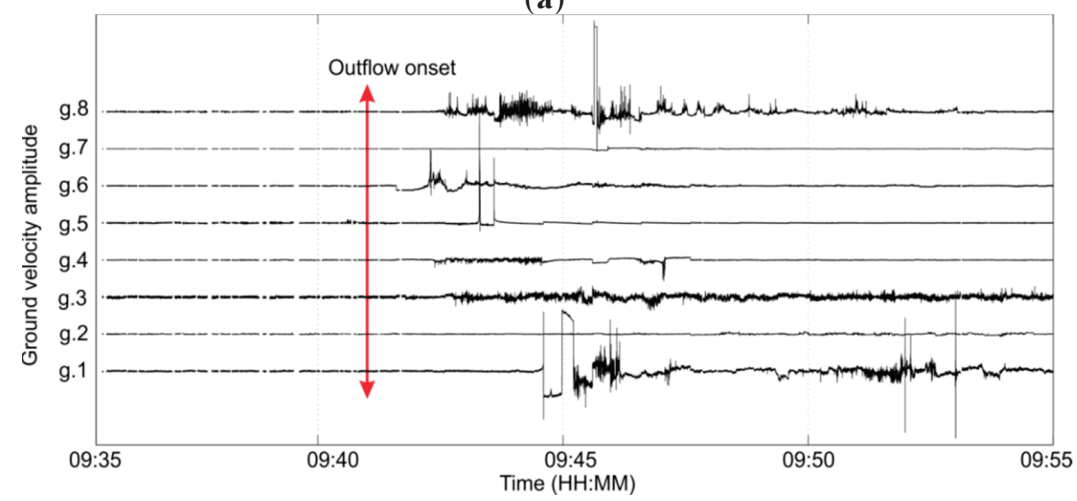

(b)

During the reported time of the enhanced $\mathrm{CO}_{2} / \mathrm{CH}_{4}$ outflow along the monitoring well (9:41), we find an increase of the noise level for seven out of the eight geophones (Figure 3a). Figure 3b shows twenty-minute waveform recordings framing the onset of the outflow. Clear differences are visible in 
the waveform signals before and after the onset of the outflow, which might indicate the arrival of the $\mathrm{CO}_{2} / \mathrm{CH}_{4}$ front at the geophone array. The increased noise levels are maintained for the remainder of the monitoring period studied. No clear preference for the outflow detection in terms of channel orientation is found. During the onset of the outflow, most of the sensors present extremely disturbed noise levels but no uniform waveform signatures can be identified. Interestingly, the arrival times of the elevated noise levels are not displaying a linear move out along the array, but in contrast they are time-delayed with no systematic order. To further analyse these signals, we investigated the pressure data measured by the sensors installed at the monitoring well (Figure 4). At the time of the onset of the outflow, the pressure at the sensor at 1,640 $\mathrm{m}$ depth decreased by $1 \mathrm{MPa}$, while the pressure in the sensor installed at $1,300 \mathrm{~m}$ increased by $300 \mathrm{kPa}$. Therefore, there was a dramatic gradient of pressure with both depth and time which subsequently recovered to the respective pre-outflow level after approximately $2 \mathrm{~h}$. The pressure gradient confirms the interpretation of the detected noise level perturbations as a signal related to the $\mathrm{CO}_{2} / \mathrm{CH}_{4}$ migration along the well.

Figure 4. (Left): Pressure measured by the sensors inside the observation well during the two-week time period analysed in this study. (Right): Zoom on the pressure perturbations at the reported time of the $\mathrm{CO}_{2}$ leakage (1 September 2005, 09:41).
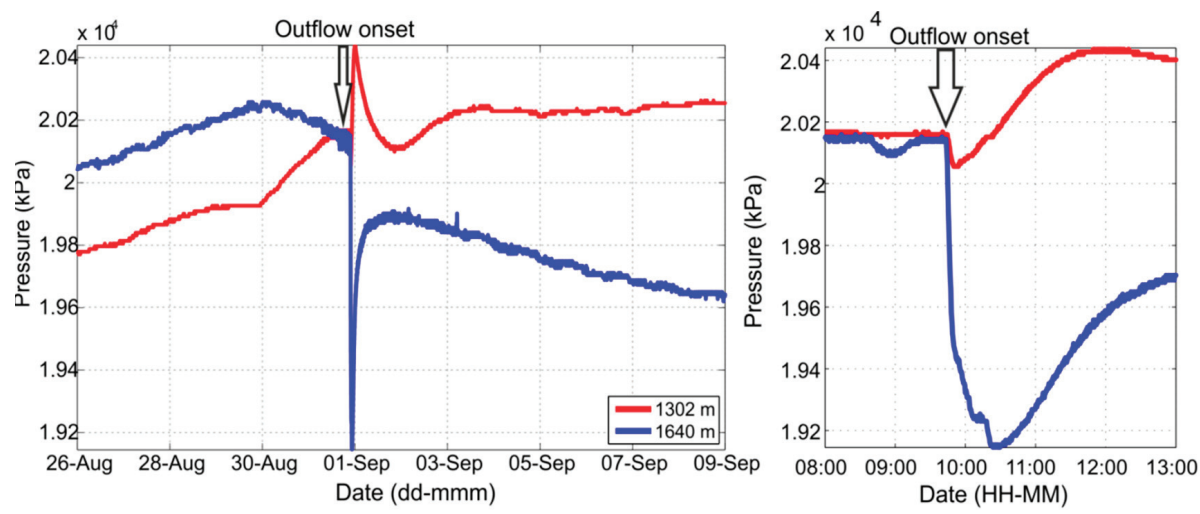

\subsection{STA/LTA Analysis}

Due to the lack of regional seismicity and since no calibration shots were available, we tuned the algorithm parameters based on the accurate detection of several different signals visually identified. Figure 5 shows a waveform data example and corresponding detections of the STA/LTA algorithm.

The resulting detections of the STA/LTA analysis were visually checked and classified into six different categories (Figure 6a) and example waveform detections are shown in Appendix A.: A-Type detections display large amplitudes at only one geophone, which suggests that the signal was a spike e.g., caused during digitization. B-Type detections typically occur close to the start or end times of periods without recordings (i.e., no seismic origin). C-Type detections display larger amplitudes at more than one, but less than four sensors. D-Type detections have extremely low SNR and thus they can be excluded of further analysis. E-Type detections belong to periods when the time series exhibit periodic-electronic signals. These signals are not introduced by the data processing, since we can observe corresponding waveforms also in the raw data. Finally, F-Type detections are signals that have high similarity between 
the different geophones. Therefore, they have a higher potential to be weak seismic events. However, these signals cannot be associated with typical induced seismicity, since it is not possible to observe $\mathrm{P}$ and $\mathrm{S}$ phases. For this reason, none of the categories actually represent clear elastic waveforms resulting from failure of rock but rather very local (in part sensor-specific) signals of different origin. Figure $6 \mathrm{~b}$ shows the daily number of detections for each type. Nearly all A-type and most of the E-Type signals occurred after the onset of the outflow. Since both types of detections might be related with electrical disturbances, they can be seen as an indicator that the leakage of $\mathrm{CO}_{2} / \mathrm{CH}_{4}$ impacted the instrumentation and/or the cables used for the data transmission to the surface. Interestingly, the highest number of Type-F events is registered on 31 August 2005, which is one day after the shut-in of $\mathrm{CO}_{2}$ injection into the reservoir (Figure 6b). It is well known that one of the periods with highest likelihood for induced micro-seismicity due to fluid injection is in the shut-in phase. This could be a reason in favour of considering Type-F events as weak induced seismicity. However, the number of events induced is rather small to be able to establish any conclusion in this respect. Additionally, on 3 September 2005, when injection was resumed, electronic spikes and spurious signals increased substantially. Consequently, the last injection might again have damaged the cabling/instrumentation resulting in increasing spurious signals. Alternatively, the recording equipment at the surface might be responsible for generating these signals (through induction or direct impact of the power net).

Figure 5. Example of waveform analyzed with STA/LTA. Upper part: filtered recordings for $20 \mathrm{~s}$ of data. The green vertical lines are the detections of the STA/LTA. Middle part: STA (black) and LTA (red) functions for the corresponding data period. Lower part: STA/LTA ratio.

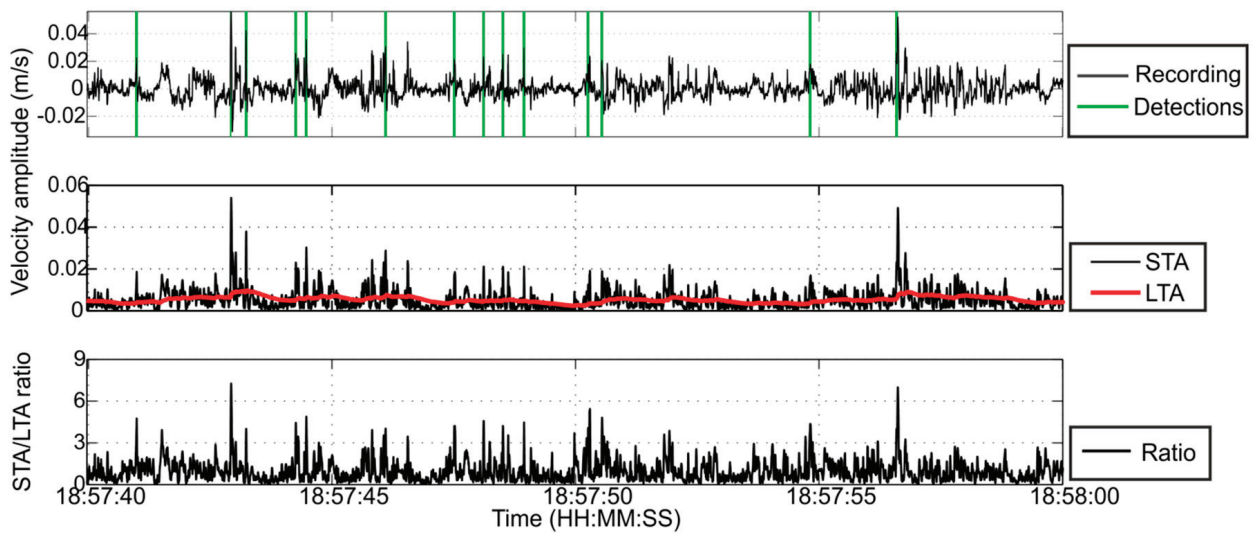

Since the geophone array was placed on average $1.6 \mathrm{~km}$ below the surface, in case of seismic events with their sources at similar depths of the instruments, they might be also recorded on the horizontal components. For this reason, we finally performed an analogous STA/LTA test also on the horizontal components. Once again, the obtained results did not reveal any waveform clearly representing induced micro-seismicity. 
Figure 6. (a) Number of STA/LTA detections of each type detected during the entire analyzed period; (b) From top to bottom: daily distribution of detection types from A to F and well-head pressure in the Injector well I1 during the two-week period.

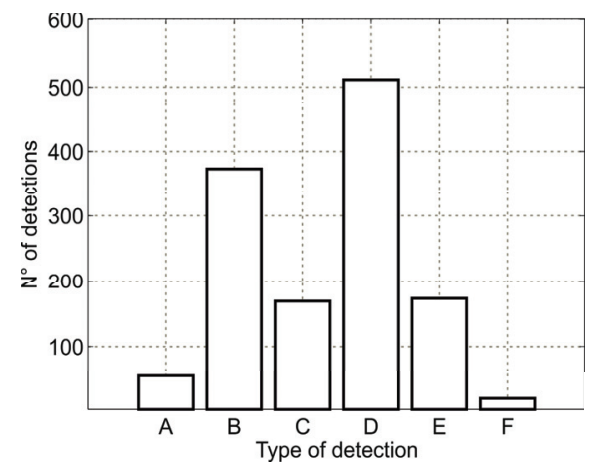

(a)

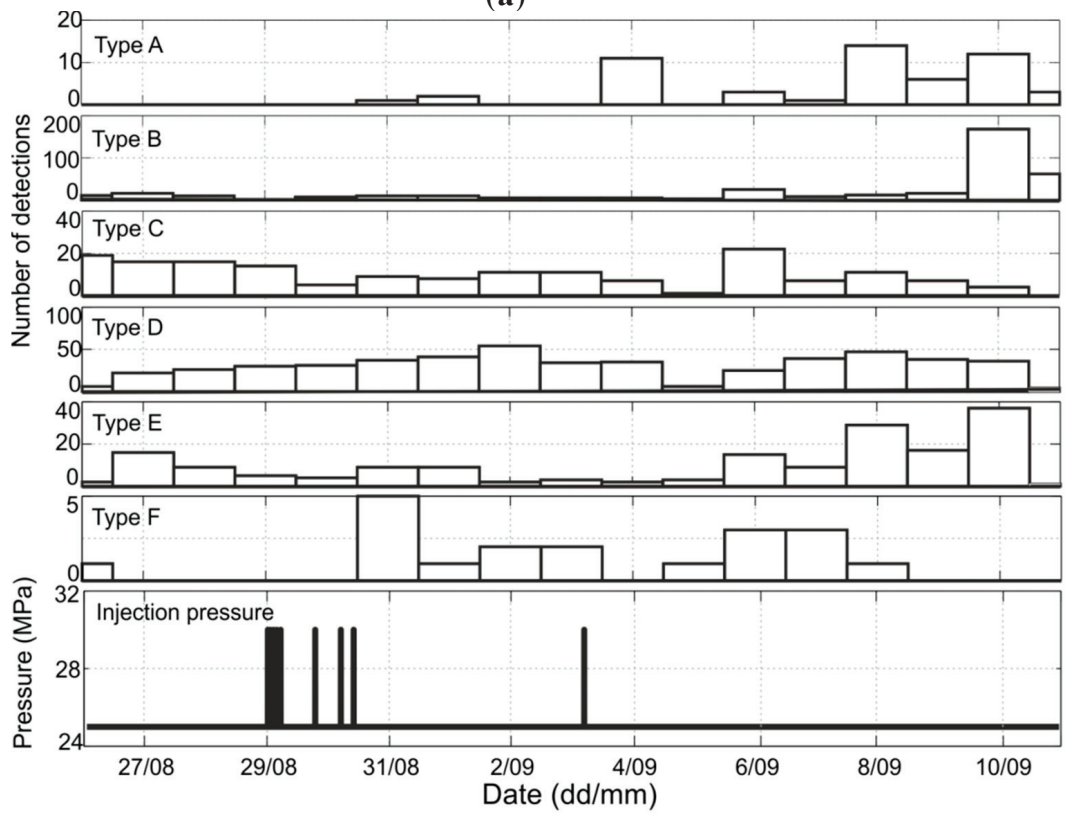

(b)

\subsection{Analysis of Low-Frequency Signals}

We found potentially relevant signals that display a similar spectral content and duration as the LPLD events (Figure 7a). However, other possible sources for these signals cannot be excluded and, after manual review of the relevant signals in each individual sensor, no coherent signal in several geophones could be identified. Given the unknown orientation of the horizontal components of each geophone, we cannot perform an analogous horizontal stack to check the consistency of these signals in other channels.

The low-frequency data processing pointed our attention towards several signals with similar waveforms of micro-earthquakes, especially following the $\mathrm{CO}_{2} / \mathrm{CH}_{4}$ outflow. Figure $7 \mathrm{~b}$ shows the 
waveform data stacking vertical components for a time-window of $100 \mathrm{~min}$ and then calculating spectrograms. As pointed out already, after the onset of the outflow (09:41), there is a clear change in the frequency content (see Section 4.1) and several signals with waveforms similar to microseismicity can be identified. However, these signals have lower frequencies than those of typical micro-seismic events, and most of them are only detected in one individual geophone. In consequence, we interpret these signals to be associated with the $\mathrm{CO}_{2} / \mathrm{CH}_{4}$ flow along the monitoring well and passing by the geophones.

Figure 7. (a) Example of signals with high similarity to LPLD events (framed by red rectangles). Upper part: stacking of the amplitudes of the vertical components. The plot has been re-filtered with a band-pass between $5-40 \mathrm{~Hz}$ to reduce electrical noise and better visualize the frequency change. Lower part: Spectrograms for the same time period; (b) Similar signals to microseismic events (framed by red rectangles). Upper part: Stack of the amplitudes for the vertical components of every geophone (100 min time window). Lower part: Spectral density stacking for the vertical components of every geophone.

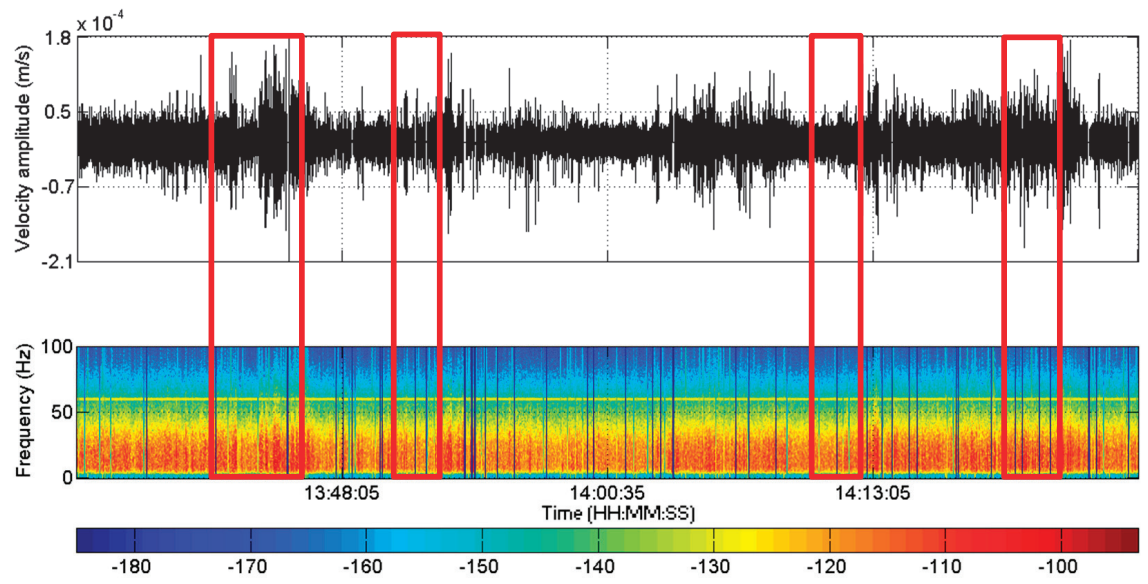

(a)
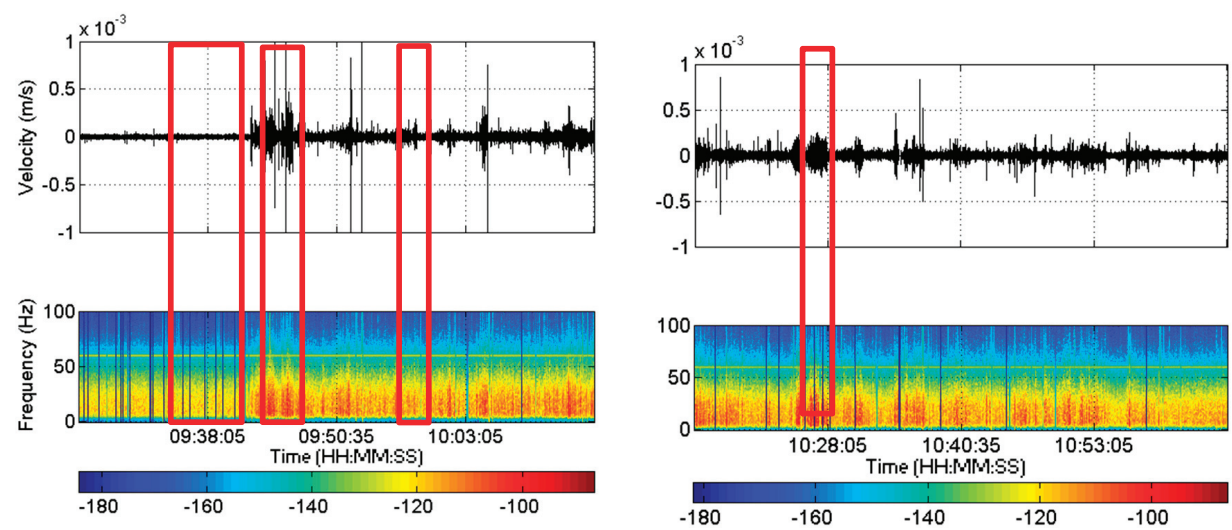

(b) 


\section{Discussion and Conclusions}

In the Pembina oil field there was a small potential for the occurrence of detectable induced seismicity due to its long production record, progressing depletion of the reservoir and the lack of (known) faults in the area. However, induced micro-seismicity might occur due to local pressure perturbations caused by $\mathrm{CO}_{2}$ injection into the reservoir and/or leakage of $\mathrm{CO}_{2}$ along the monitoring well. In the present study, our primary objective was to investigate potentially occurring $\mathrm{CO}_{2}$-induced seismic signatures focusing on a two-week period framing a substantial $\mathrm{CO}_{2}$ and $\mathrm{CH}_{4}$ leakage along the monitoring well.

The seismological techniques applied to the continuous seismic data recordings did not result in detection of any signal clearly associated with micro-seismic events with source size greater than a few meters. The most promising Type-F events detected with the STA/LTA analysis do not reflect sufficiently strong signals, and therefore it was not possible to determine their source location or to perform any further analysis. The potential LPLD signals identified with the low-frequency analysis cannot be considered as micro-seismicity either since they were detected mainly on one sensor.

As a consequence of the lack of induced seismicity reported, our study is in good agreement with the general view that fluid injection in sedimentary formations tends to trigger less seismicity than injection in crystalline rocks. This statement, of course, is subjected to the volume of injected $\mathrm{CO}_{2}$.

The most striking observation from our analysis is that we clearly identified signals related to the outflow of $\mathrm{CO}_{2} / \mathrm{CH}_{4}$ in that seven out of the eight geophones show an elevated noise level and complex signals during the reported onset. The fact that these signals are not occurring simultaneously leaves open several questions related to the actual processes triggering these signals. Since no preference in terms of channel detection was found for the elevated noise, and given the observed complexity of the signals, the origin of the elevated noise could be partially electronic disturbances introduced by the front of $\mathrm{CO}_{2} / \mathrm{CH}_{4}$ arriving to the sensors and damaging certain components.

In some studies, seismic signals associated with the drastic volume increase of $\mathrm{CO}_{2}$ during its phase change from supercritical to gaseous have been reported [17,30]. For the Penn West Pilot Project at the Pembina Field, the thermal gradient and reservoir pressure were $2.9^{\circ} \mathrm{C} / 100 \mathrm{~m}$ and $2.10 \mathrm{MPa}$, respectively [31], and therefore it is reasonable to believe that the phase change of the $\mathrm{CO}_{2}$ and the resulting elastic waves would occur at depth levels around 1,000 $\pm 100 \mathrm{~m}$. Since in our case the geophone array was placed much deeper (1,500-1,640 m) and given the small energy of such signals, the current location of the monitoring equipment would not allow detecting them, although they might have occurred in the context of the outflow. This conclusion, however, is restricted to $\mathrm{CO}_{2}$, while any similar behaviour for the $\mathrm{CH}_{4}$ would need to be evaluated in detail separately.

Due to the $\mathrm{CO}_{2} / \mathrm{CH}_{4}$ leakage reported along the observation well, the data from our borehole geophone array provide valuable hints about processes related to the migration of $\mathrm{CO}_{2}$. This case study is a good example to illustrate the importance of performing an appropriate deployment of the instrumentation and a good preparation of the data acquisition system in order to obtain reliable and correct data for the reservoir monitoring. 


\section{Acknowledgments}

We acknowledge funding within the Helmholtz-Alberta Initiative (HAI), from the Helmholtz foundation in the framework of the Helmholtz Young Investigator Group 'From microseismicity to large earthquakes' and from the EU-GEISER project. We thank Georg Dresen and the three anonymous reviewers for their constructive comments and feedback.

\section{Conflict of Interest}

The authors declare no conflict of interest.

\section{References}

1. Intergovernmental Panel on Climate Change (IPCC). Mitigation of Climate Change. In Climate Change: Geneva, Switzerland, 2007. Available online: http:www.ipcc.ch/ipccreports/ar4-wg3.htm (accessed on 30 August 2013).

2. Schrag, D.P. Preparing to capture carbon. Science 2007, 315, 812-813.

3. Verdon, J.P.; Kendall, J.M.; White, D.J.; Angus, D.A. Linking microseismicity event observations with geomechanical models to minimize the risks of storing $\mathrm{CO}_{2}$ in geological formations. Earth Planet. Sci. Lett. 2011, 305, 143-152.

4. Verdon, J.P.; Kendall, J.M. Detection of multiple fracture sets using observations of shear-wave splitting in microseismic data. Geophys. Prospect. 2011, 59, 593-608.

5. Maxwell, S.C.; Urbancic, T.I.; Devon, E.N.S.; Schlumberger, R.Z. Microseismic Imaging of Hydraulic Fracture Complexity in the Barnett Shale. In Proceedings of the SPE Annual Technical Conference and Exhibition, San Antonio, TX, USA, 29 September-2 October 2002.

6. Rutledge, J.T.; Phillips, W.S.; Roff, A.; Albright, J.N.; Hamilton-Smith, T.; Jones, S.; Kimmich, K. Subsurface Fracture Mapping Using Microearthquakes Detected during Primary Oil Production, Clinton County, Kentucky. In Proceedings of Annual Technical Conference and Exhibition, New Orleans, LA, USA, 25-28 September 1994.

7. Majer, E.L.; Baria, R.; Stark, M.; Oates, S.; Bommer, J.; Smith, B.; Asanuma, H. Induced seismicity associated with Enhanced Geothermal Systems. Geothermics 2007, 36, 185-222.

8. Shapiro, S.A. Microseismicity: A Tool for Reservoir Characterization, Education Tour Series CIS; EAGE Publications: Houten, The Netherlands, 2008.

9. Bohnhoff, M.; Dresen, G.; Ellsworth, W.L.; Ito, H. Passive Seismic Monitoring of Natural and Induced Earthquakes: Case Studies, Future Directions and Socio-Economic Relevance. In New Frontiers in Integrated Solid Earth Sciences, (International Year of Planet Earth); Springer: Berlin/Heidelberg, Germany, 2010; pp. 261-285.

10. Kwiatek, G.; Bohnhoff, M.; Dresen, G.; Schulze, A.; Schulte, T.; Zimmermann, G.; Huenges, E. Microseismicity induced during fluid-injection: A case study from the geothermal site at Gross Schoenebeck, North German Basin. Acta Geophys. 2010, 58, 995-1020.

11. Angus, D.A.; Verdon, J.P. Using microseismicity to estimate formation permeability for geological storage of $\mathrm{CO}_{2}$. Geophysics 2013, doi:10.1155/2013/160758. 
12. Verdon, J.P.; Wuestefeld, A. Measurement of the normal/tangential compliance ratio (ZN/ZT) during hydraulic fracture stimulation using shear wave splitting data. Geophys. Prospect. 2013, doi:10.1111/j.1365-2478.2012.01132x.

13. Zoback, M.; Gorelick, S.M. Earthquake triggering and large-scale geologic storage of carbon dioxide. Proc. Natl. Acad. Sci. USA 2012, doi:10.1073/pnas.1202473109.

14. Bachu, $\mathrm{S}$. Sequestration of $\mathrm{CO}_{2}$ in geologic media in response to climate change: Road map for site selection using the transform of the geological space into the $\mathrm{CO}_{2}$ phase space. Energy Conserv. Manag. 2002, 43, 277-289.

15. Krause, F.F.; Collins, H.N.; Nelson, D.A.; Machemer, S.D.; French, P.R. Multiscale anatomy of a reservoir: Geological characterization of pembina-cardium pool, West-Central Alberta, Canada. Am. Assoc. Pet. Geol. 1987, 71, 1233-1260.

16. Evans, K.F.; Zappone, A.; Kraft, T.; Deichmann, N.; Moia, F. A survey of the induced seismic responses to fluid injection in geothermal and $\mathrm{CO}_{2}$ reservoirs in Europe. Geothermics 2011, 41, 30-54.

17. Bohnhoff, M.; Zoback, M.D.; Chiaramonte, L.; Gerst, J.L.; Gupta, N. Seismic detection of $\mathrm{CO}_{2}$ leakage along monitoring wellbores. Int. J. Greenh. Control 2010, 4, 687-697.

18. Verdon, J.P.; Kendall, J.M.; White, D.J.; Angus, D.A.; Fisher, Q.J.; Urbancic, T. Passive seismic monitoring of carbon dioxide storage at Weyburn. Lead. Edge 2010, 29, 200-206.

19. Oye, V.; Aker, E.; Daley, T.M.; Kühn, D.; Bohloli, B.; Korneev, V. Microseismic monitoring and interpretation of injection data from the In Salah $\mathrm{CO}_{2}$ storage site (Krechba), Algeria. Energy Procedia 2013, 37, 4191-4198.

20. Verdon, J.P.; Kendall, J.M.; Stork, A.L.; Chadwick, R.A.; White, D.J.; Bissell, R.C. Comparrison of geomechanical deformation induced by megatonne-scale $\mathrm{CO}_{2}$ storage at Sleipner, Weyburn, and In Salah. Proc. Natl. Acad. Sci. USA 2013, doi:10.1073/pnas.1302156110.

21. Gunter, W.D. Pembina Cardium Summary Report; Penn West Energy Trust: Calgary, AB, Canada, 2008.

22. Hitchon, B. Pembina Cardium $\mathrm{CO}_{2}$ Monitoring Pilot. A CO $2-E O R$ Project, Alberta, Canada; Geoscience Publishing: Edmonton, AB, Canada, 2009.

23. Zambrano-Narvaez, G.; Chalaturnyk, R.J. Design and Deployment of an Integrated Instrumentation System in a Monitoring Well at the Penn West $\mathrm{CO}_{2}$-EOR Pilot, Alberta, Canada. In Proceedings of the 7th International Symposium on Field Measurements in Geomechanics, Boston, MA, USA, 24-27 September 2007.

24. Zambrano-Narvaez, G.; Chalaturnyk, R.J. Case study of the cementing phase of an observation well at the Pembina Cardium $\mathrm{CO}_{2}$ monitoring pilot, Alberta, Canada. Int. J. Greenh. Gas Control 2011, 5, 841-849.

25. Kwiatek, G.; Plenkers, K.; Dresen, G. Source parameters of Picoseismicity Recorded at Mponeng Deep Gold Mine, South Africa: Implications for scaling relations. Bull. Seismol. Soc. Am. 2011, 101, 2592-2608.

26. Coueslan, M.L. Processing and Interpretation of Time-Lapse Vertical Seismic Profile Data from the Penn West $\mathrm{CO}_{2}$ Monitoring Project. M.Sc. Thesis, University of Calgary, Calgary, AB, Canada, April 2007.

27. Evans, J.; Allen, S. A teleseism-specific detection algorithm for single short period traces. Bull. Seism. Soc. Am. 1983, 73, 1173-1186. 
28. Das, I.; Zoback, M.D. Long-period, long-duration seismic events during hydraulic fracturing gas stimulation of a shale gas reservoir. Lead. Edge 2011, 30, 778-786.

29. Eaton, D.W.; van der Baan, M.; Tary, J.B.; Birkelo, B.; Cutten, S. Low-Frequency Tremor Signals from a Hydraulic Fracture Treatment in Northeast British Columbia, Canada. In Proceedings of the 4th EAGE Passive Seismic Workshop, Amsterdam, The Netherlands, 17-20 March 2013.

30. Bohnhoff, M.; Zoback, M. Oscillation of fluid-filled cracks triggered by degassing of $\mathrm{CO}_{2}$ due to leakage along wellbores. J. Geophys. Res. 2010, 115, doi:10.1029/2010JB000848.

31. Zambrano-Narvaez, G. Design, Deployment, Performance and Assessment of Downhole and Near Surface Monitoring Technology for Geological $\mathrm{CO}_{2}$ Storage. Ph.D. Thesis, University of Alberta, Edmonton, AB, Canada, March 2012.

\section{Appendix A}

Figure A1. Examples of signals identified by the STA/LTA observed during the two-week time period analysed in this study. Each subfigure shows the vertical components of geophones 1 to 8 from bottom to up. Date of the detection appears in the top of each plot (dd/mm/yyyy). Each trace is normalized by the maximum of and interval which covers $0.1 \mathrm{~s}$ before and after the detection. (a-c): Examples of detections from Type-F events. (d): Type-A event with the largest amplitude registered in geophone g.3. (e) Type-B event at the edge of a period without recording (typical recording for the last days of the two-week period). (f) Type-E event in which periodic signals appear in $\mathrm{g} .8$.

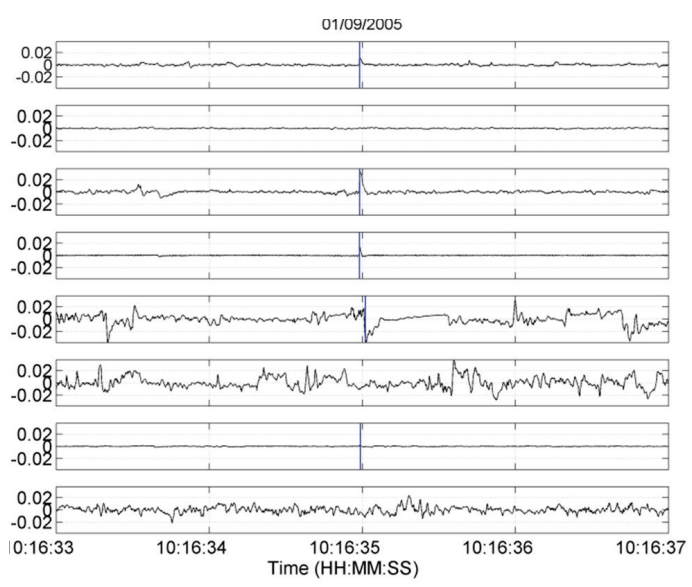

(a)

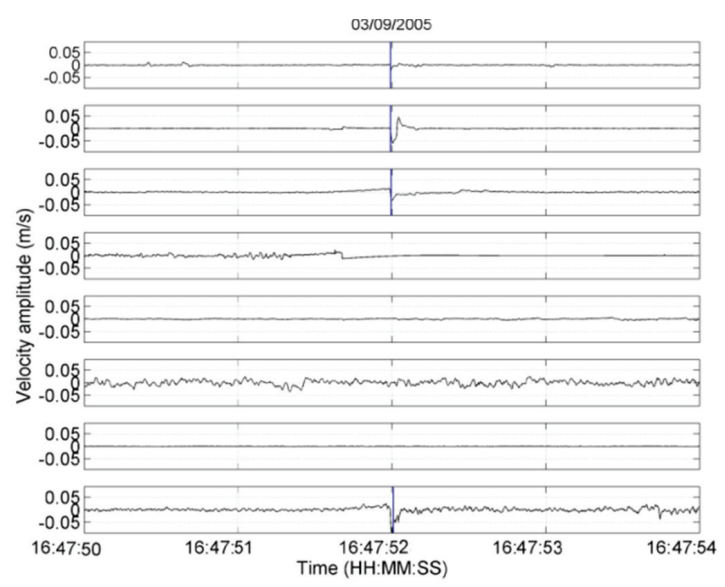

(b) 
Figure A1. Cont.
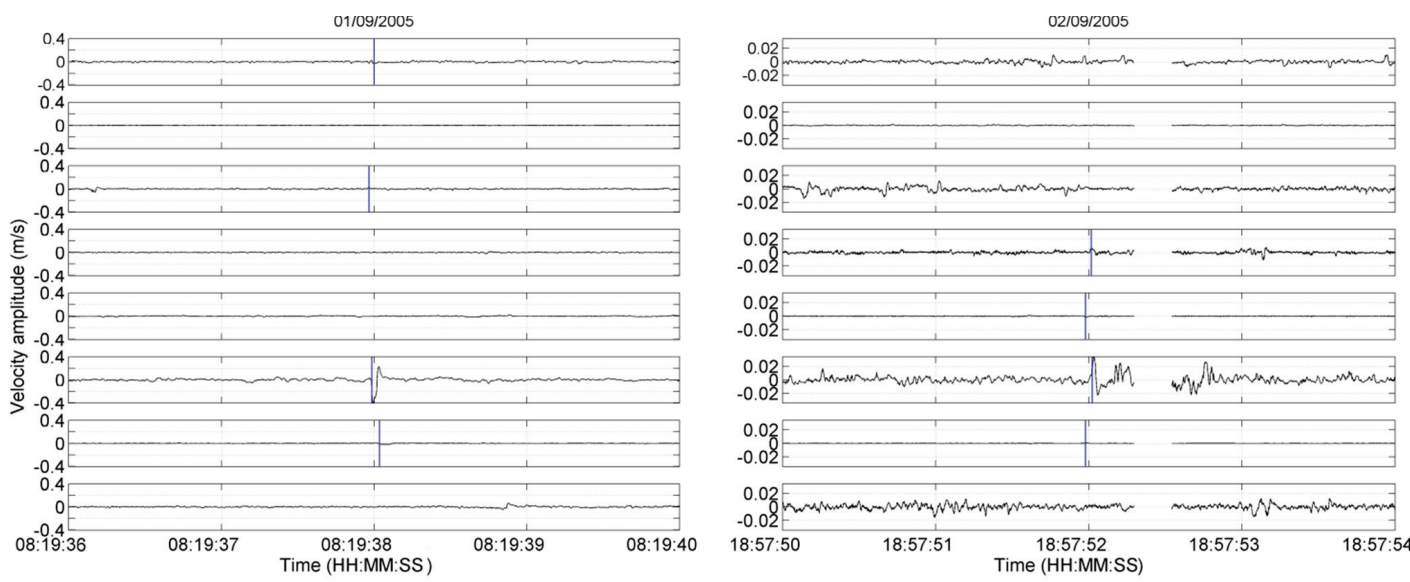

(c)

(d)

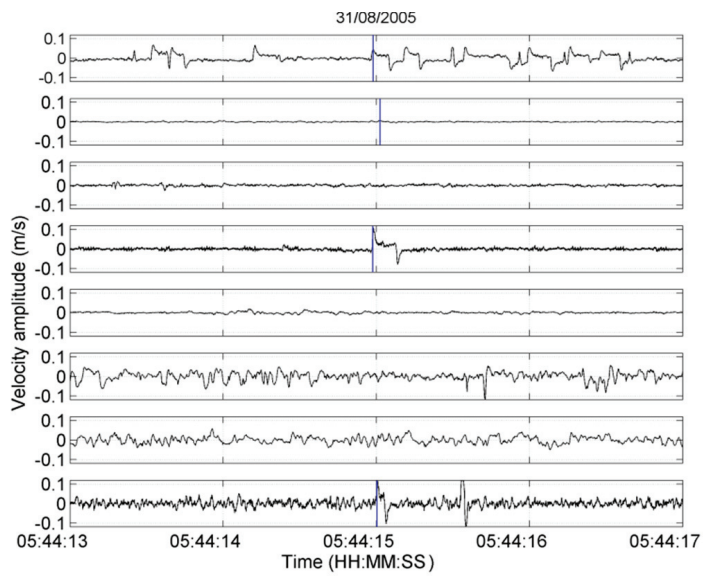

(e)

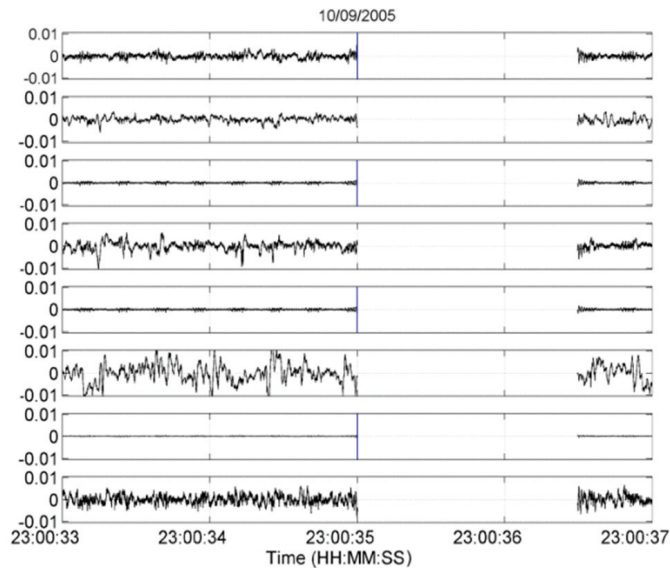

(f) 


\title{
Thermal Tracking in Mobile Robots for Leak Inspection Activities
}

\section{Aitor Ibarguren, Jorge Molina, Loreto Susperregi and Iñaki Maurtua}

\begin{abstract}
Maintenance tasks are crucial for all kind of industries, especially in extensive industrial plants, like solar thermal power plants. The incorporation of robots is a key issue for automating inspection activities, as it will allow a constant and regular control over the whole plant. This paper presents an autonomous robotic system to perform pipeline inspection for early detection and prevention of leakages in thermal power plants, based on the work developed within the MAINBOT (http://www.mainbot.eu) European project. Based on the information provided by a thermographic camera, the system is able to detect leakages in the collectors and pipelines. Beside the leakage detection algorithms, the system includes a particle filter-based tracking algorithm to keep the target in the field of view of the camera and to avoid the irregularities of the terrain while the robot patrols the plant. The information provided by the particle filter is further used to command a robot arm, which handles the camera and ensures that the target is always within the image. The obtained results show the suitability of the proposed approach, adding a tracking algorithm to improve the performance of the leakage detection system.
\end{abstract}

Reprinted from Sensors. Cite as: Ibarguren, A.; Molina, J.; Susperregi, L.; Maurtua, I. Thermal Tracking in Mobile Robots for Leak Inspection Activities. Sensors 2013, 13, 13560-13574.

\section{Introduction}

Efficient and effective maintenance is crucial for all kinds of industries. In the case of capital intensive investment industries, such as petrochemicals, the steel industry or power generation plants, it is even more relevant and has an important impact on the operation costs during the long lifecycle of their production means.

Automating inspection activities in industrial plants, especially in extensive plants, poses strong requirements from different points of view: a huge number of elements to inspect (pipes, valves, switches, pumps, vessels, motors, vibrating machinery, chillers, ovens, etc.), handling multiple sensors or special non-destructive testing equipment to be used (visual, ultrasonic, vibration, radiography, thermography, eddy current, noise analysis, gas sensors, etc.) and extensive production facilities that spread out for thousands of square meters, either in the vertical or horizontal, and risky working conditions for maintenance personnel, due to the presence of hazardous materials.

This paper presents part of the work performed in the MAINBOT European project. This project aims at developing service robot applications to autonomously execute inspection tasks in extensive industrial plants. The objective is to develop a surveillance robotic system able to detect the leakage of fluids using a vision system in the thermal and visible ranges. To validate the proposed solution, a solar plant of cylindrical-parabolic collectors is used, testing the approach in a very demanding environment from a mobile manipulation point of view. 
The paper is organized as follows. Section 2 gives information about related works. Section 3 introduces the inspection task to be performed by the system. Section 4 presents the proposed approach, the architecture for leak inspection in solar thermal plants and autonomous navigation. Sections 5 and 6 are devoted to the leakage detection algorithm and the tracking system, respectively. In Section 7, the experimental results of the system are shown. Finally, Section 8 poses the obtained results, as well as the future work to be done.

\section{Related Work}

Robots have been used for maintenance tasks in a wide range of applications and environments. From preventive maintenance of high-voltage transmission power lines [1] to inspection of cables [2] and nuclear reactor pressure vessels [3] in the nuclear industry, autonomous systems are used in many industries in an attempt to improve maintenance tasks. Focusing on pipeline inspection, Suzuki et al. [4] propose an autonomous robot for industrial pipeline inspection by means of ultrasonic diagnosis equipment. In the same way, Camerini et al. [5] present an underwater inspection robot for offshore pipeline inspection, using the pipeline itself for guidance purposes. Even so, a few works add some tracking tools as particle filters to improve the inspection task.

Several works have also been performed using thermal images for tracking and detection. Jiping et al. [6] propose a target detection and tracking system based on different morphological operations. Senthil Kumar et al. [7] pose the fusion of thermal images with 2D images for tracking Unmanned Aerial Vehicles (UAVs), adding optical flow techniques, such as LucasâĂŞKanade and HornâĂŞSchunck methods. Finally, Padole and Alexandre [8] propose the use of particle filters for human tracking with thermal images, using motion information to feed the particle filter. The presented work also proposes the use of particle filtering, although it uses the whole image information and dynamics to feed the particle filter, not only the motion information.

\section{Task Specification}

Valle 1 and 2 are two solar thermal power plants, whose promoter and owner is Torresol Energy Investments, S.A., and which are located in San José del Valle (Spain); see Figure 1. Valle 1 and 2 are two adjacent solar thermal power plants that generate electricity by means of cylindrical-parabolic collectors. 
Figure 1. Valle 1 and 2 solar thermal power plants.
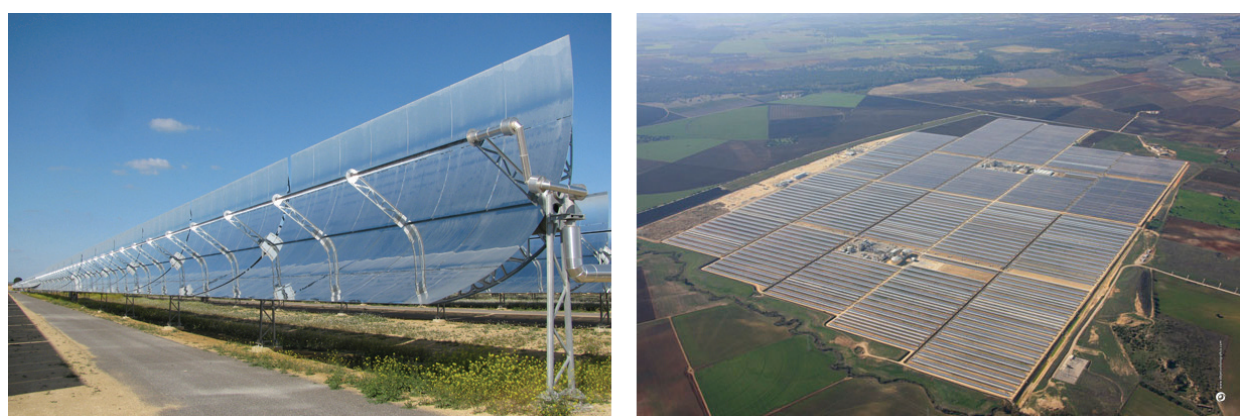

The solar field is composed of nearly 7,500 parabolic cylinder collectors. These collectors transport a Heat Transfer Fluid (HTF), which absorbs the solar energy. HTF circulates at a high temperature (around $390{ }^{\circ} \mathrm{C}$ ) inside the absorber tubes, which is used after to heat the molten salts to generate steam in the Steam Generation System (SGS).

Swivel joints are critical points where leakages may happen (this is the point where the collector tube connects with the infrastructure of pipes that are deployed all over the plant). HTF leakages are not desirable, as oil losses may be unsafe, due to the high temperatures reached in the solar power plant. Early detection and prevention of leakages is a key issue for the maintenance of those kinds of facilities. Even so, the huge area of solar power plants makes it difficult to perform proper maintenance, due to the large amount of kilometers of collectors and pipelines, as well as the hazardous environment with the really high temperatures reached in its elements.

Nowadays, the inspection is performed by human operators using a thermographic camera while they travel along different parts of the solar plant by car through poorly asphalted and dirt roads. The inspection is carried out while the vehicle is moving, looking at the screen to analyze the thermal image and detecting leakages. Even so, the operator must inspect the image for long time periods (around $2 \mathrm{~h}$ ) while correcting the the pose of the camera to keep the pipeline in the field of view when irregularities in the terrain appear, which may lead to leakages being left undetected.

\section{Proposed Approach}

Based on the task specifications posed in the previous section, this paper proposes an autonomous robotic system to perform the pipeline inspection for early detection and prevention of leakages. The autonomous robot patrols along the power plant while inspecting the collectors using thermographic images to identify the leakages. The inspection system continuously analyzes the images in order to monitor the status of the elements, the tube in this case, and highlight anomalies.

Initially, a path is defined for the robotic platform based on a hybrid map approach mixing topological graphs with local occupancy grids. Using the information provided by a GPS/IMU sensor, the robot executes the planned path. During this execution, the system takes advantage of a local metric planner to avoid obstacles and follows the initial path as close as possible. 
Even so, irregularities in the terrain (poorly asphalted road and dirt road) make it difficult to perform the inspection using a fixed camera mounted on the robot, as the pipeline can be out of range when slopes and bumps are found on the road. Taking this into account, the addition of a robotic arm is proposed to allow the manipulation of the thermal camera and to track the pipeline through the inspection task. The aim is to establish a coordination between the thermographic inspection and the robot arm movements in order to keep the objective in the field of view of the camera.

The next sections will give information about the used hardware and system architecture, including the different software units defined within the system and the navigation system.

\subsection{Architecture}

The architecture presented in this paper is based on the specifications and work performed in the MAINBOT project. The main robotic platform used is a RobucarTT developed by Robosoft (http://www.robosoft.com/), designed for outdoor environments and with the Ackermann steering geometry. The robot includes a GPS/IMU sensor for localization purposes, which provides the robot position with an accuracy of $0.20 \mathrm{~m}$. The platform also has a robotic arm attached to it, which is employed in this case to handle a thermographic camera, specifically, an FLIRThermoVision ${ }^{\circledR}$ A20. This thermographic camera is used for leakage detection, as well as for tracking purposes. Figure 2 shows the physical elements of the architecture.

Figure 2. Robotic platform and thermographic camera.
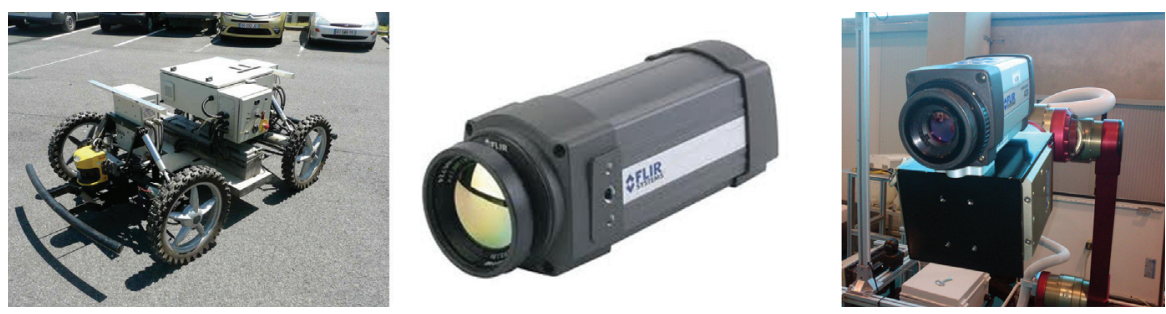

There are two main tasks to be performed by the robotic platform while the predefined path is being executed by the robotic platform: on the one hand, to inspect the pipelines to search for leakages; on the other hand, to track the pipeline in order to send movements to the robotic arm and to maintain the pipe in the field of view of the camera. To this end, as shown in Figure 3, two different modules have been defined in the software architecture:

- Leak inspection unit: the unit in charge of receiving images from the thermographic camera and analyzing them in order to detect leakages in the collectors. Based on different morphological operations in the image, the unit is able to detect the leakages in the collectors in an accurate way.

- Thermal tracking unit: the unit in charge of tracking the pipeline and commanding the robotic arm to keep the pipe in the field of view of the camera. A particle filter-based tracking system 
is proposed, because to its capacity to accurately model the underlying dynamics and its rapid adaptation to changing signal features.

Figure 3. Software architecture: leak inspection unit and thermal tracking unit.

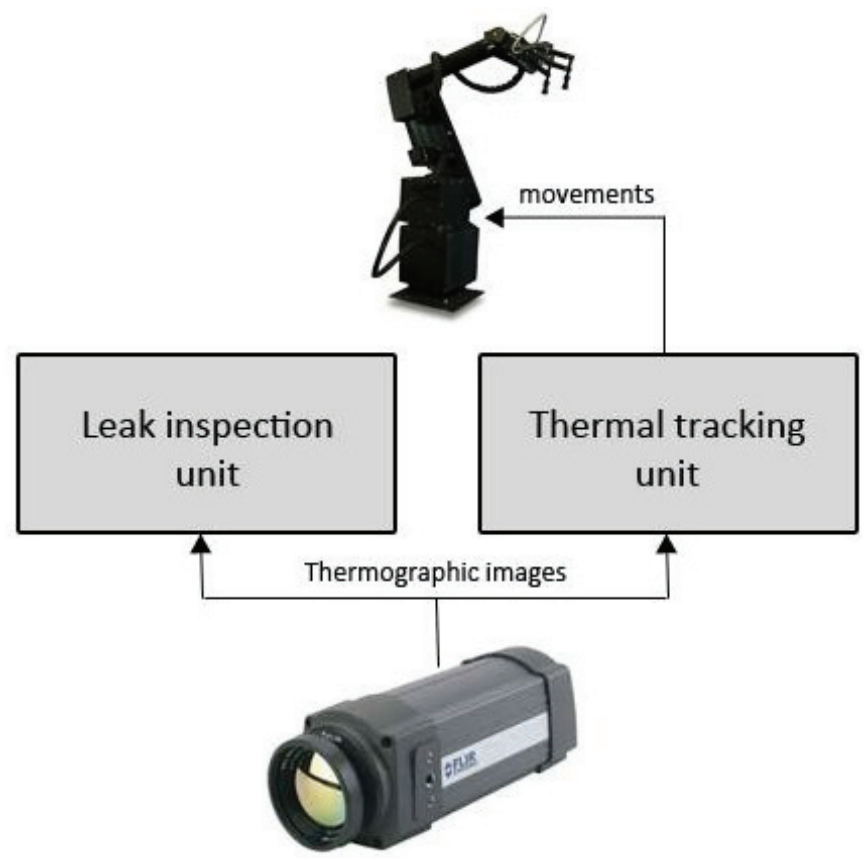

Based on this architecture, the system is able to (a) inspect the pipelines and detect leakages and (b) maintain stable the detection process by means of the tracking process, overcoming the problems derived from the irregularities of the terrain, while the mobile robot executes the planned path for inspection.

\subsection{Autonomous Navigation}

The autonomous navigation approach of the MAINBOT project is based on the use of a hybrid map consisting of a topological graph overlaid with local occupancy grids. Since the workspace is a large area, the overall plan is formed on a topological graph, as planning in a large metric map quickly becomes unwieldy. However, local metric information is used for achieving precise localization (needed for some operations) and obstacle avoidance. Hence, the path planning is performed in two steps:

- The overall plan is created in the topological graph, using Dijkstra's algorithm. This is the basis for the low level planning.

- The robot navigates locally using local metric maps and a search-based planning algorithm. 
- The global metric planner integrated in MAINBOT generates a path from the current position to a desired goal by combining a series of short, kinematically feasible "motion primitives". Planning is done in $\mathrm{x}, \mathrm{y}$ and theta dimensions, resulting in smooth paths that take robot orientation into account. This is especially important for a RobucarTT robot, as it has nonholonomic constraints (i.e., due to the Ackermann configuration).

- The local metric planner can be seen as a controller that drives a mobile base in the plane.

- An execution component called "move base" links the global and local planners to achieve the metric navigation.

This navigation approach allows for creating paths for pipeline inspection in two steps, using the "move base" component to execute the defined path as accurately as possible. The autonomous navigation module has been developed using ROS (http://www.ros.org) (Robot Operating System) libraries and packages.

\section{Leak Inspection Unit}

The aim of this unit is the detection of leakages in the collectors based on information provided by a thermographic camera. To this end, initially, (a) the image is analyzed, searching for a pipeline section. Once the pipeline has been found; (b) the section is inspected to detect abrupt changes in the temperature, which indicate that there are leakages in the collectors.

In this process, as the first step, the object parameters of the thermal camera must be fixed. To extract the temperature information from an image, the output data of the thermal camera must be interpreted based on the correct fixing of parameters, such as emissivity, object distance or reflected temperature.

The emissivity is a surface property that states the ability to emit energy; it is expressed as the ratio of the radiation emitted by a surface to the radiation emitted by a blackbody. Emissivity is a unitless quantity and spans from zero to one.

Following the energy conservation law, all energy exchange is compensated mutually: the flux incident, $\Phi_{i}$, is equal to the flux reflected, $\Phi_{r}^{\prime}$, absorbed, $\Phi_{a}$, and transmitted, $\Phi_{t}$ :

$$
\Phi_{i}=\Phi_{r}^{\prime}+\Phi_{a}+\Phi_{t}
$$

In general cases, terms on the right side of Equation (1) are specifically weighted following particular radiative properties related to reflection $(\rho)$, absorption $(\alpha)$ and transmission $(\tau)$.

These properties are linked together considering the flux exchanges on a semitransparent object in its environment for which:

$$
\rho+\alpha+\tau=1
$$

The general form of Kirchhoffêt's law provides a link between the absorption and emission processes and, thus, between emissivity and absorbance, since:

$$
\varepsilon\left(\lambda, \theta^{\prime}, \phi^{\prime}\right)=\alpha(\lambda, \theta, \phi)
$$

The object distance is defined as the distance from the camera to the surface. Finally, reflected temperature indicates the temperature reflected by the surface of an object. 
In the case of the glass that covers the collector, it has a high transmissivity, around $94 \%(0.94)$, so the emissivity is fixed to 0.04 . The estimated reflected temperature is fixed empirically to $10{ }^{\circ} \mathrm{C}$ and the object distance to $10 \mathrm{~m}$, based on the features of the environment and the solar plant.

Once the settings are established, the camera processes the thermal information and provides an image where pixels give information about the temperature. These values of temperature are normalized to gray values performing a thermal adjustment between the minimum and maximum values defined (in our case, between $100^{\circ} \mathrm{C}$ and $300^{\circ} \mathrm{C}$ ), as shown in Figure 4A.

Figure 4. (A) Thermographic image; (B) Pipeline detection; and (C) Leak detection.

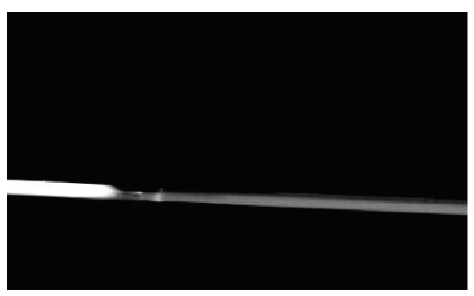

(A)

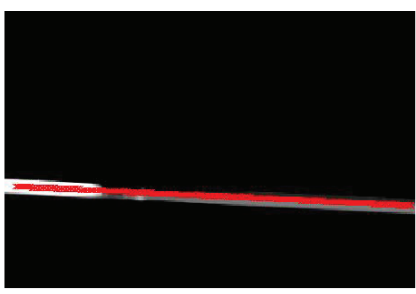

(B)

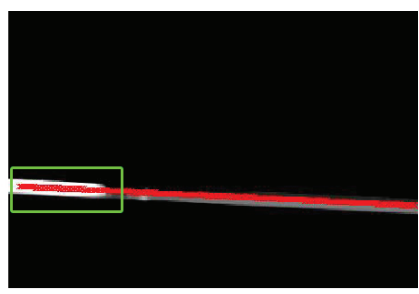

(C)

This image is then thresholded, highlighting the pixels with temperatures above $100{ }^{\circ} \mathrm{C}$, as they are related with the pipelines. Skeletonization/Medial Axis Transform [9] is applied to this image, detecting the longest straight section in the image, as illustrated in Figure 4B. This straight section is then analyzed to detect temperature changes along the pipeline.

As the first step to detect the temperature changes along the collector, the temperatures of the previously obtained section are stored, as shown in Figure 5A, where the temperature along the collector is plotted. Based on these data, the absolute value of the first derivative is calculated as:

$$
A B S\left(T^{\prime}\right)=\left|T_{i+1}-T_{i}\right|, \quad i=[1 \ldots N-1]
$$

where $T_{i}$ is the temperature of pixel $i$ of the pipeline section.

The information about the absolute value of the derivative is used to divide the section into different parts, which have similar temperature, defining a $\Delta T$ threshold (minimum change) to perform this division. An important consideration is the fact that the joints of different sections of the collector are metallic and have a high reflectivity, so the obtained temperature is unstable at this point and must be filtered. In order to filter them, an approximate resolution of the image is estimated and short parts with abrupt change of temperature are removed, defining a minimum width of the pipeline part. This allows us to filter short pipeline parts with abrupt temperature changes (joints), detecting real leakages, which fill a wider space on the image. Once the pipeline is divided, the mean temperature of each subsection is computed. The pipeline parts above the maximum temperature are labeled as leakages, as highlighted in green in Figures $4 \mathrm{C}$ and 5C. 
Figure 5. (A) Temperature along the pipeline; (B) Absolute value of the first derivative; and $(\mathbf{C})$ Detection on the plot.

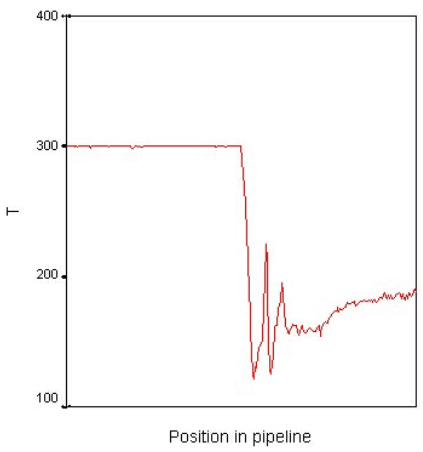

(A)

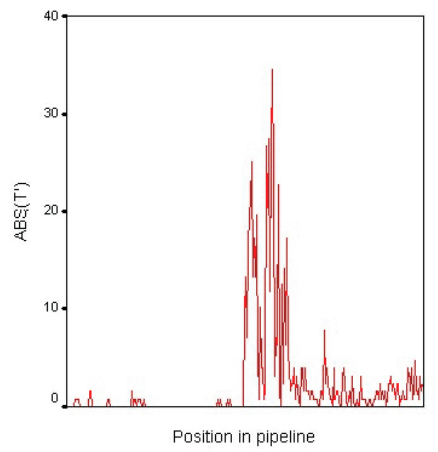

(B)

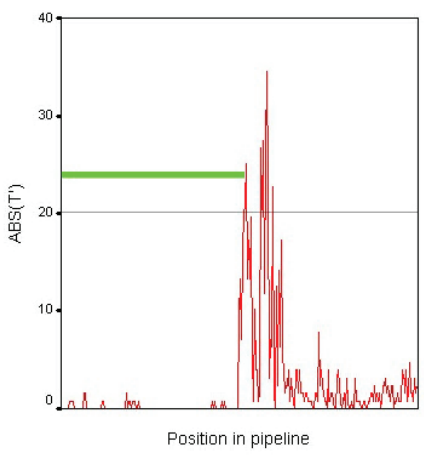

(c)

Based on this algorithm, the Leak Detection Unit is able to raise alerts when leakages are detected while the robot is patrolling along the collectors.

\section{Thermal Tracking Unit}

Irregularities in the terrain can make it difficult to perform a correct leak inspection, as pipelines can be out of the field of view of the camera when slopes and bumps are found along the road. To overcome this problem, a tracking system is proposed based on particle filtering. This tracking system follows the target pipeline through thermographic image sequences, and it is able to generate movement commands when the target is reaching the edge of the image. The next lines will give information about all the elements of the particle filter for thermal tracking.

\subsection{Particle Filter}

Particle filters [10,11], also known as Sequential Monte Carlo methods (SMC), are sequential estimation techniques that allow estimating unknown states, $x_{t}$, from a collection of observations $z_{1: t}=\left\{z_{1}, \ldots, z_{t}\right\}$. The state-space model is usually described by state transition and measurement equations:

$$
\begin{gathered}
x_{t}=f_{t}\left(x_{t-1}, v_{t-1}\right) \\
z_{t}=g_{t}\left(x_{t}, u_{t}\right)
\end{gathered}
$$

where $f$ and $g$ are the state evolution and observation model functions, respectively, and $v_{t}$ and $u_{t}$ denote the process and observation noise, respectively.

Based on the previous equations, particle filters allow for approximating the posterior density (PDF) by means of a set of particles, $\left\{x_{t}^{(i)}\right\}_{i=1, \ldots, n}$, using equation:

$$
p\left(x_{t} \mid z_{1: t}\right)=\sum_{i=1}^{N} \omega_{t}^{(i)} \delta\left(x_{t}-x_{t}^{(i)}\right)
$$


where each particle, $x_{t}^{(i)}$, has an importance weight, $\omega_{t}^{(i)}$, associated with it and $\delta$ is the Kronecker delta. These weights are computed following equation:

$$
\omega_{t}^{(i)}=\omega_{t-1}^{(i)} \frac{p\left(z_{t} \mid x_{t}^{(i)}\right) p\left(x_{t}^{(i)} \mid x_{t-1}^{(i)}\right)}{q\left(x_{t}^{(i)} \mid x_{0: t-1}^{(i)}, z_{0: t}\right)}
$$

where $p\left(z_{t} \mid x_{t}^{(i)}\right)$ is the likelihood function of the measurements, $z_{t}$, and, finally, $q\left(x_{t}^{(i)} \mid x_{0: t-1}^{(i)}, z_{0: t}\right)$ is the proposal density function.

Based on the previously presented equations, the particle set evolves along time, changing the weights of the particles and resampling them in terms of the observations.

Particle filtering provides a robust tracking framework when dealing with non-linear and non-Gaussian state and observation functions, as it considers multiple state hypotheses simultaneously.

\subsection{Particle Filtering for Thermal Tracking}

In the first step, a tracking process has been defined in an attempt to maintain the object to be analyzed in the field of view of the thermal camera. A particle filter-based tracking is proposed, allowing us to correct the position of the robotic arm to maintain the pipe in the center of the image for a further analysis. In an attempt to develop a thermal tracking system for multiple detection tasks, the particle filter has been generalized to allow the tracking of objects with different shapes and configurable for each tracking process (easy reconfiguration for similar scenarios), although the system modeling explained in the next lines is tuned for the presented environment and posed problem.

\subsubsection{System Modeling}

In the presented scenario, the tracking process is able to follow a pipeline in successive frames. The state of the process is defined as:

$$
X_{t}=\left[x_{t}, y_{t}, l_{t}, \alpha_{t}\right]^{T}
$$

where $x_{t}$ and $y_{t}$ are the $x$ and $y$ pixel coordinates of the center of the pipeline in the image, $l_{t}$ is the length of the pipeline and $\alpha_{t}$ is the orientation of the pipeline in time $t$.

Additionally, the state transition is defined as:

$$
\begin{aligned}
X_{t+1} & =X_{t}+\Delta_{t} \dot{X}_{t}+V_{t} \\
\dot{X}_{t} & =\left[\dot{x}_{t}, \dot{y}_{t}, \dot{l}_{t}, \dot{\alpha}_{t}\right]^{T}
\end{aligned}
$$

where $\Delta_{t}$ is the time step, $\dot{X}_{t}$ is the dynamic part describing the variation of the state elements and $V_{t}$ is an additive, zero mean Gaussian noise. 


\subsubsection{Likelihood Evaluation}

For the likelihood evaluation, initially, the thermal image is analyzed to highlight the parts with a predefined temperature range, in this case, the temperature of the pipe to be tracked. To this end, thresholding and Skeletonization/Medial Axis Transform algorithms have been used, as in the leak inspection unit. From this step, a set of $N$ connected regions are extracted; $N$ possible pipe sections. Those regions form the observation, $Z_{t}$, where each region, $z_{i}^{t}$, is defined by their center in pixel coordinates, length and angle:

$$
\begin{gathered}
Z_{t}=z_{i=1 . . N}^{t} \\
z_{i}^{t}=\left[x_{i}^{t}, y_{i}^{t}, l_{i}^{t}, \alpha_{i}^{t}\right]^{T}
\end{gathered}
$$

For the likelihood evaluation, initially, the distance between the particle and each of the observed regions is calculated using the pixel coordinates of the center, length and angle as:

$$
\text { dist }_{i}=\lambda \sqrt{\left(x_{t}-x_{i}^{t}\right)^{2}+\left(y_{t}-y_{i}^{t}\right)^{2}}+\beta\left|l_{t}-l_{i}^{t}\right|^{2}+\gamma\left|\alpha_{t}-\alpha_{i}^{t}\right|^{2}
$$

where $\lambda, \beta$ and $\gamma$ are coefficients to weigh the importance of the pixel coordinates, length and angle, respectively. The distance between the particle and the observation is then calculated as the minimum distance between the particle and the $N$ regions found:

$$
\operatorname{dist}\left(X_{t}, Z_{t}\right)=\min \left(\operatorname{dist}_{i}\right), \quad i=[1 . . N]
$$

Finally, the likelihood is calculated as the exponential of the distance, as shown in the next equation:

$$
P\left(Z_{t} \mid X_{t}\right)=e^{-\operatorname{dist}\left(X_{t}, Z_{t}\right)}
$$

Based on the presented likelihood evaluation, the particle filter estimates iteratively the process state as presented in the next paragraphs.

\subsubsection{Particle Filtering Procedure}

To initialize the process, when the first pipe is detected, a set of $\mathrm{N}$ random particles is drawn around its position and with its scale and orientation. Afterwards, the procedure of the particle filter is given as:

- Find the object in the initial thermal image and initialize $\mathrm{N}$ particles, $X_{0}^{(i)}$, with random samples around it, where $w_{0}^{(i)}=1 / N$;

- If ESS < threshold (effective sample size), draw $\mathrm{N}$ samples with selection with replacement;

- Predict $x_{t}^{(i)}=x_{t-1}^{(i)}+v_{t-1}$;

- Update importance weights $w_{t}^{(i)}=w_{t-1}^{(i)} P\left(Z_{t} \mid X_{t}\right)$; 5: Normalize weights $w_{t}^{\left(i^{\prime}\right)}=$ $w_{t}^{(i)} / \sum_{j=1}^{N} w_{t}^{(j)}$;

- Set $t=t+1$, go to Step 2 .

In this procedure, ESS [12] (effective sample size) is calculated as:

$$
c v_{t}^{2}=\frac{\operatorname{var}\left(w_{t}^{(i)}\right)}{E^{2}\left(w_{t}^{(i)}\right)}=\frac{1}{N} \sum_{i=1}^{N}\left(N w_{t}^{(i)}-1\right)^{2}
$$




$$
E S S_{t}=\frac{N}{1+c v_{t}^{2}}
$$

where $N$ is the number of particles and $w_{t}^{(i)}$ is the weight of particle $i$ in time $t$.

Based on this discrete approximation of the posterior probability, the object is tracked along the inspection process.

\section{Experimental Results}

To test the suitability of the proposed approach, a set of experiments have been designed trying to asses both the leakage detection algorithm and the tracking system. To this end, a database of image sequences was created in Valle facilities (see Figure 6 ) using actual production means and replicating the behavior of the maintenance robot:

- Recording of collectors and pipelines using a thermal camera $10 \mathrm{~km}$ at night, as performed now by human operators, divided in sequences of $150 \mathrm{~m}$ (half loop of collectors).

- Camera placed on a vehicle circulating at a speed of $20 \mathrm{~km} / \mathrm{h}$ through a terrain with irregularities.

- Real leakages appearing in the images.

Based on this real data, two different experiments have been performed. The next lines give information about each experiment and the obtained results.

Figure 6. Sequence of thermographic images on Valle facilities.
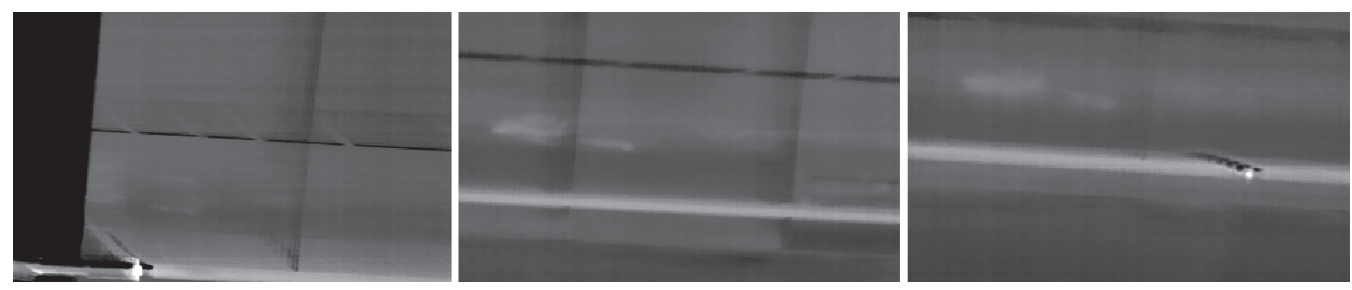

\subsection{Results of the Leak Detection}

An efficiency of the leak detection has been tested using 60 different thermal sequences. In those 60 sequences, human operators found a total of seven leakages during the recording session, which were labeled in the database. Those 60 sequences were analyzed by the previously presented algorithm, searching for leakages. A threshold, $\Delta T$, of $70{ }^{\circ} \mathrm{C}$ was established for the detection algorithm empirically based on the gathered data. In order to have numeric data, a mean value for each stretch of the tube with temperature change (filtering joints) was also saved.

Figure 7 shows an example of the output of the algorithm while performing the validation test, where each column is related with a sequence. The first row indicates the image sequence ID, while the next rows show the mean temperature of the pipe section along the collectors where abrupt changes of temperature can be observed. Pipe parts with a high temperature (leakages) are marked in red. 
Figure 7. Example of changes in temperature in recorded image sequences.

\begin{tabular}{|c|c|c|c|c|c|c|c|c|c|c|c|c|}
\hline P1A2 & P1A4 & P1A5 & P1A7 & P1A8 & P1A10 & P1A11 & P1A12 & P1A16 & P1A24 & P1A25 & P1A28 & P1A35 \\
\hline 136,07 & 136,07 & 143,13 & 135,29 & 142,35 & 160,39 & 156,47 & 147,84 & 134,5 & 142,35 & 129,8 & 128,23 & 149,41 \\
\hline 132,15 & 132,15 & 132,15 & 158,82 & 132,15 & 142,35 & 137,64 & 143,92 & 142,35 & 138,43 & 158,03 & 119,6 & 133,72 \\
\hline 127,45 & 127,45 & 132,15 & 299,21 & 144,7 & 174,5 & 189,41 & 153,33 & 172,15 & 246,66 & 207,45 & 271,76 & 138,43 \\
\hline 299,21 & 299,21 & 192,54 & 298,43 & 193,33 & 260,78 & 202,74 & 292,94 & 237,25 & 217,64 & 189,41 & 138,43 & 205,88 \\
\hline 293,72 & 293,72 & 299,21 & 300 & 292,94 & 249,8 & 272,54 & 252,15 & 238,03 & 132,94 & 122,74 & 138,43 & 209,01 \\
\hline 290,58 & 290,58 & 296,07 & 298,43 & 246,66 & 152,54 & 152,54 & 260 & 170,58 & 129,01 & 122,74 & & 139,21 \\
\hline 292,94 & 292,94 & 141,56 & 296,07 & 217,64 & 148,62 & 154,11 & 141,56 & 146,27 & & & & 125,09 \\
\hline 150,19 & 150,19 & 135,29 & 299,21 & 252,15 & & & 138,43 & 152,54 & & & & 128,23 \\
\hline 140 & 140 & 136,07 & 299,21 & 136,07 & & & 139,21 & & & & & \\
\hline 139,21 & 139,21 & & 148,62 & 125,88 & & & & & & & & \\
\hline & $\ldots$ & & 145,49 & & & & & & & & & \\
\hline & & & & & & & & & & & & \\
\hline & 148,62 & & & & & & & & & & & \\
\hline & 143,13 & & & & & & & & & & & \\
\hline & 179,21 & & & & & & & & & & & \\
\hline & 258,43 & & & & & & & & & & & \\
\hline & 263,92 & & & & & & & & & & & \\
\hline & 263,13 & & & & & & & & & & & \\
\hline & 243,52 & & & & & & & & & & & \\
\hline & 143,13 & & & & & & & & & & & \\
\hline & 144,7 & & & & & & & & & & & \\
\hline
\end{tabular}

The algorithm found all the leakages labeled by human operators, obtaining $100 \%$ sensitivity. Besides, six more leakages were found along the sequences, leakages that match exactly with the previous leakage patterns. Based on the similarity and after an analysis of the new leakages, they could be considered as real leaks that were missed by the human operators during visual inspection.

\subsection{Results of the Tracking Process}

To test the efficiency of the particle filter for tracking, 10 different image sequences have been used, each of them with 200 images approximately (a total of 2,000 images). For each sequence, the particle filter has been initialized using the first pipeline appearance, and the pipeline has been tracked through the rest of the images. In this experiment, the manipulation of the robotic arm has been left out of the scope of this manuscript, as the sequences have been recorded by a human operator from a car and it is not possible to simulate the arm movements. For each frame, the error between the output of the particle filter and the labeled images has been computed, as shown in Figure 8. Images show peaks in the error plots, derived from the image noise and irregularities in the terrain.

Figure 8. Tracking error during a recorded sequence in position in $\mathrm{X}$ and $\mathrm{Y}$ orientation and length.
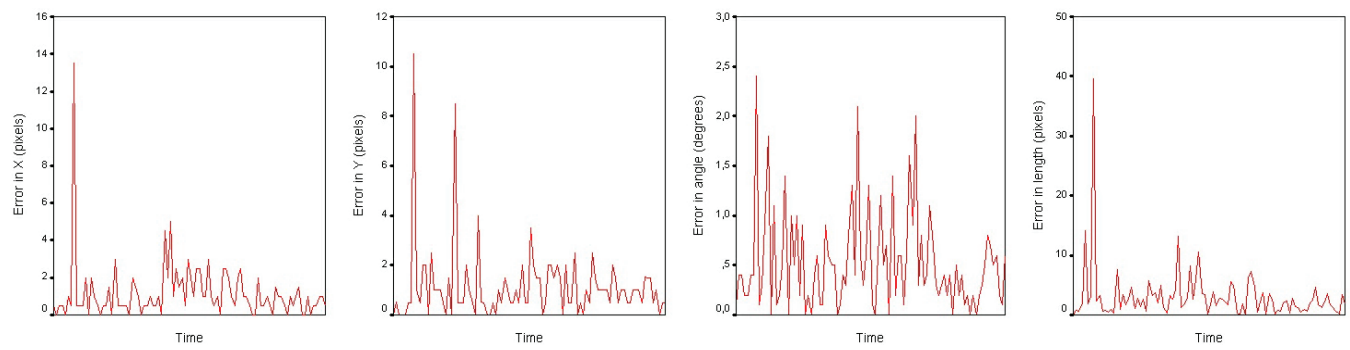
Specifically, the experiment measures the tracking error in position, angle and length of the pipeline, using different parameters for the particle filter. Those are the specifications of the experiment:

- Six different particle filter configurations have been set up, mixing different state estimation methods and numbers of particles. Specifically, the state estimation methods are:

- Best particle (the one with maximum weight);

- Robust mean with the 10 particles with the maximum weight;

- Weighted mean using the whole particle set;

- For each configuration, the previously cited image sequences have been used, tracking the pipeline through around 2,000 images; the mean error and standard deviation of the position, angle and pipe length has been measured for all the configurations;

- In the likelihood evaluation step, the same values for coefficients $\lambda, \beta$ and $\gamma$ have been used, applying values that give a similar weight to position, angle and length.

Table 1 shows the obtained results. The first column describes the number of particles and the estimation method, the second and third columns, the mean $(\mu)$ and the standard deviation $(\sigma)$ of the error in the position and the fourth and fifth columns, the mean $(\mu)$ and the standard deviation $(\sigma)$ of the error in the angle, and finally, the last columns show the mean $(\mu)$ and the standard deviation $(\sigma)$ of the error in the length.

Table 1. Results of the Tracking Process.

\begin{tabular}{|c|c|c|c|c|c|c|}
\hline & \multicolumn{2}{|c|}{ Pos. Error (pix) } & \multicolumn{2}{|c|}{ Angle Error $\left(^{\circ}\right)$} & \multicolumn{2}{|c|}{ Length Error (pix) } \\
\hline & $\mu$ & $\sigma$ & $\mu$ & $\sigma$ & $\mu$ & $\sigma$ \\
\hline 500 - Best particle & 1.80 & 1.27 & 0.96 & 0.86 & 3.16 & 2.58 \\
\hline 1,000 - Best particle & 1.42 & 0.95 & 0.72 & 0.63 & 2.94 & 2.51 \\
\hline 500 - Robust mean 10 & 1.82 & 2.11 & 0.51 & 0.41 & 2.86 & 5.35 \\
\hline 1,000 - Robust mean 10 & 1.57 & 1.83 & 0.41 & 0.34 & 2.65 & 5.66 \\
\hline 500 - Weighted mean & 6.34 & 5.39 & 0.43 & 0.36 & 3.67 & 7.68 \\
\hline 1,000 - Weighted mean & 6.11 & 4.67 & 0.35 & 0.38 & 3.45 & 6.64 \\
\hline
\end{tabular}

Results show a better performance of the particle filter with a set of 1,000 particles, improving the mean error and standard deviation in almost all the configurations. The addition of a bigger set of particles could decrease the error rates, although it would be necessary to optimize the computational time as much as possible to ensure a suitable frame rate. In the case of the state estimation methods, the best particle and robust mean methods show very similar results, overcoming in any case the errors of the weighted mean. 


\section{Conclusions and Future Work}

This paper presents an autonomous leakage detection system for maintenance tasks in extensive industrial plants, including a leakage detection system. Based on the information provided by a thermographic camera, the system is able to detect leakages in pipelines and collectors of thermal power plants. This system is enhanced by a particle filter-based tracking system, used to maintain the target in the field of view of the camera and stabilize the detection process when irregularities are found along the road.

The results show a high success rate of the leakage detection unit, reaching $100 \%$ sensitivity (on data labeled by operators) and detecting even more leakages on the recorded sequences. The use of thermographic information allows for detecting the fluids leaked from the collectors, taking advantage of morphological operations to highlight the leaks in the thermal images.

Besides, a tracking system has been added to manage the thermographic camera and to avoid the loss of the target. The particle filtering for tracking has shown a position error of less than 1.5 pixels and less than a $0.5^{\circ}$ error in the angle. It also allows for modeling the system in a simple and effective way, adapting rapidly to the changing image features and data noise.

As further work, there are two main paths to follow: on the one hand, to add the robot arm movements and to mix the information extracted by the leak inspection unit and thermal tracking unit with the navigation module to create a complete system that takes into account the navigation information in the inspection task and vice versa. In the same way, it would also be interesting to test the tracking system in similar inspection tasks where different elements of the solar plant are analyzed (valves, vessels, etc.) and observe its suitability.

\section{Acknowledgments}

This work has been performed within the scope of the project "MAINBOT: Mobile robots for inspection and maintenance activities in extensive industrial plants". MAINBOT is a small or medium-scale focused research project supported by the European Commission in the 7th Framework Program (285245). For further information, see http://www.mainbot.eu.

\section{Conflicts of Interest}

The authors declare no conflict of interest.

\section{References}

1. Debenest, P.; Guarnieri, M.; Takita, K.; Fukushima, E.F.; Hirose, S.; Tamura, K.; Kimura, A.; Kubokawa, H.; Iwama, N.; Shiga, F.; et al. Expliner-Toward a Practical Robot for Inspection of High-Voltage Lines. In Proceedings of the Field and Service Robotics (FSR), Cambridge, MA, USA, 14-16 July 2009; pp. 45-55. 
2. Lee, J.K.; Cho, B.H.; Jang, K.N.; Jung, S.C.; Oh, K.Y.; Park, J.Y.; Kim, J.S. Development of Autonomous Cable Inspection Robot for Nuclear Power Plant. In Proceedings of World Academy of Science, Engineering and Technology, Rome, Italy, 28-30 April 2010; pp. 314-318.

3. Luk, B.L.; Collie, A.A.; Cooke, D.S; Chen, S. Walking and Climbing Service Robots for Safety Inspection of Nuclear Reactor Pressure Vessels. In Proceedings of the Asia Pacific Conference on Risk Management and Safety, Hong Kong, 1-2 December 2005; pp. 432-438.

4. Suzuki, M.; Yukawa, T.; Satoh, Y.; Okano, H. Mechanisms of Autonomous Pipe-Surface Inspection Robot with Magnetic Elements. In Proceedings of the IEEE International Conference on Systems, Man and Cybernetics, Taipei, 8-11 October 2006; pp. 3286-3291.

5. Camerini, C.; Freitas, M.; Langer, R.A.; von Der Weid, J.P.; Marnet, R.; Kubrusly, A.C. A Robot for Offshore Pipeline Inspection. In Proceedings of the 9th IEEE/IAS International Conference on Industry Applications (INDUSCON), Sao Paulo, Brazil, 8-10 November 2010; pp. 8-10.

6. Xu, J.; Ikram-ul-haq; Chen, J.; Dou, L.; Liu, Z. Moving Target Detection and Tracking in FLIR Image Sequences Based on Thermal Target Modeling. In Proceedings of the International Conference on Measuring Technology and Mechatronics Automation (ICMTMA), Changsha, China, 13-14 March 2010; pp. 715-720.

7. Senthil Kumar, K.; Kavitha, G.; Subramanian, R.; Ramesh, G. Visual and Thermal Image Fusion for UAV Based Target Tracking. In MATLAB-A Ubiquitous Tool for the Practical Engineer; Ionescu, C.M., Ed.; InTech: Rijeka, Croatia, 2001; pp. 307-326.

8. Padole, C.N.; Alexandre, L.A. Motion Based Particle Filter for Human Tracking with Thermal Imaging. In Proceedings of the 3rd International Conference on Emerging Trends in Engineering and Technology (ICETET 2010), Goa, India, 19-21 November 2010; pp. 158-162.

9. Aichholzer, O.; Aurenhammer, F. Straight Skeletons for General Polygonal Figures in the Plane. In Proceedings of the 2nd Annual International Conference on Computing and Combinatorics, Hong Kong, 17-19 June 1996; pp. 117-126.

10. Doucet, A.; de Freitas, N.; Gordon, N. Sequential Monte Carlo Methods in Practice; Springer: New York, NY, USA, 2001.

11. Kotecha, J.H.; Djuric, P.M. Gaussian Particle Filtering. IEEE Trans. Signal Process. 2003, 51, 2592-2601.

12. Liu, J.S.; Chen, R.; Logvinenko, T. A theoretical Framework for Sequential Importance Sampling and Resampling. In Sequential Monte Carlo Methods in Practice; Springer: New York, NY, USA, 2001; pp. 225-246. 


\title{
Optimal Sensor Placement for Leak Location in Water Distribution Networks Using Genetic Algorithms
}

\author{
Myrna V. Casillas, Vicenç Puig, Luis E. Garza-Castañón and Albert Rosich
}

\begin{abstract}
This paper proposes a new sensor placement approach for leak location in water distribution networks (WDNs). The sensor placement problem is formulated as an integer optimization problem. The optimization criterion consists in minimizing the number of non-isolable leaks according to the isolability criteria introduced. Because of the large size and non-linear integer nature of the resulting optimization problem, genetic algorithms (GAs) are used as the solution approach. The obtained results are compared with a semi-exhaustive search method with higher computational effort, proving that GA allows one to find near-optimal solutions with less computational load. Moreover, three ways of increasing the robustness of the GA-based sensor placement method have been proposed using a time horizon analysis, a distance-based scoring and considering different leaks sizes. A great advantage of the proposed methodology is that it does not depend on the isolation method chosen by the user, as long as it is based on leak sensitivity analysis. Experiments in two networks allow us to evaluate the performance of the proposed approach.
\end{abstract}

Reprinted from Sensors. Cite as: Casillas, M.V.; Puig, V.; Garza-Castañón, L.E.; Rosich, A. Optimal Sensor Placement for Leak Location in Water Distribution Networks Using Genetic Algorithms. Sensors 2013, 13, 14984-15005.

\section{Introduction}

Leaks in water distribution networks are an issue of great concern for water utilities, strongly linked with operational costs and water resources savings. Continuous improvements in water loss management are being applied, and new technologies are developed to achieve higher levels of efficiency [1].

The traditional approach to leakage control is a passive one, whereby the leak is repaired only when it becomes visible. Recently, developed acoustic instruments [2] allow one to also locate invisible leaks, but unfortunately, their application over a large-scale water network is very expensive and time-consuming. A viable solution is to divide the network into a district metered area (DMA), where the flow and the pressure are measured, and to maintain a permanent leakage control system [3]. Then, leak detection in the DMA consists of monitoring flows at night, when customers demand is low and the leakage component is at its largest percentage over the flow. Therefore, practitioners monitor the DMA or groups of DMAs for detecting (and then repairing) leakages by analyzing the minimum night flow and, also, employ techniques to estimate the leakage level [1].

Regarding leak location methods for DMAs, several works have been proposed in the literature. For example, a review of transient-based leak detection methods is offered in [4] as a summary of current and past work. Model-based leak detection and isolation techniques have also been studied, starting with the seminal paper of Pudar and Liggett [5], which formulates the leak detection and isolation problem as a least-squares estimation problem. However, in such non-linear models, the 
parameter estimation of the water networks is not an easy task. Alternatively, in [6], a method based on pressure measurements and leak sensitivity analysis is proposed. This methodology consists in analyzing the residuals on-line, i.e., the difference between the measurements and their estimation using the network hydraulic models, regarding a given threshold that takes into account the model uncertainty and the noise. When some of the residuals violate their threshold, they are correlated against the leak sensitivity matrix in order to discover which possible leak is present. Although this approach presents satisfactory results under ideal conditions, its performance decreases in the presence of nodal demand uncertainty and noise in the measurements. An improved technique has recently been developed [7,8], where an extended time horizon analysis of pressure measurements is considered and a comparison between the performances depending on the metric used is performed.

Thus, the development of a sensor placement strategy has become an important research issue in recent years. Ideally, a sensor network should be configured to facilitate fault detection and maximize leak location performance. However, it is obvious that only a limited number of sensors can be installed inside a DMA, due to budget constraints. The main objectives of sensor placement are leak detectability, isolability and localization. Leak detectability is the ability of monitoring a variation in pressure due to a loss of water occurring in the network. Leak isolability concerns the capacity of distinguishing between two possible leak occurrences, whereas leak localization refers to finding the node where the leak is occurring. There are some works devoted to sensor placement for fault detection and isolation (FDI). Some approaches propose to locate sensors based on isolability criteria according to the study of structural matrices [9]. In [10], an optimization method based on binary integer linear programming searches for an optimal set of sensors for model-based FDI.

Each of the previously mentioned works is used in the general framework of FDI of dynamic systems. However, there are several contributions dedicated to sensor placement in water distribution networks. Most of the works have addressed the sensor placement problem regarding contamination monitoring. See, for example [11,12]; the problem of deploying sensors in a large water distribution network is considered in order to detect the malicious introduction of contaminants. On the other hand, less work has been done regarding sensor placement for leak location. In [13], a strategy based on isolability maximization allows one to optimally locate sensors for leak location based on the structural model of water network. Closer to our research, in [6], an optimal sensor placement for leak location is formulated as an integer programming problem. Recently, in [14], an entropy-based approach for the purposes of efficient and economically viable water loss incident detection was presented.

This paper proposes a new approach for sensor placement for leak location in DMAs that can be used with the projection-based location scheme proposed in [7,8]. The proposed approach is different from the algorithm presented in [6], since no binarization of the leak sensitivity matrix is used, being able to work directly using the numerical expression of this matrix. As shown in [7,8], leak isolation methods that use the leak sensitivity matrix without binarization lead to better performance. In particular, the method that computes the projection (angle) between the observed residuals and the columns of the leak sensitivity matrix is the one that performs the best. For this reason, the sensor placement approach developed in this paper uses the non-binarized leak sensitivity matrix and the projection-based leak isolation approach. The use of the non-binarized leak sensitivity matrix 
leads to completely reformulating the optimization problem solved by the algorithm presented in [6], since the isolability conditions will be completely different. In a binary context, two leaks are isolable if the corresponding columns of the binarized leak sensitivity matrix are different. However, in a non-binarized context, such criteria should be reformulated, as will be shown in this paper. As in [6], the sensor placement problem is finally formulated as an integer optimization problem. However, now, the optimization criterion consists in minimizing the number of non-isolable leaks according to the isolability criteria introduced. Because of the large size and non-linear integer nature of the resulting optimization problem, genetic algorithms (GAs) are used as the solution approach. The obtained results are compared with a semi-exhaustive search method with a higher computational cost, proving that GA allows one to find near-optimal solutions with less computational load. Another advantage is that the proposed sensor placement methodology does not depend on the isolation method chosen by the user, as long as it is based on leak sensitivity analysis. Moreover, since the numerical expression of the leak sensitivity matrix is used and this sensitivity depends on the leak size, among other factors, a robust sensor placement approach is also proposed. Experiments in two networks allow for evaluating the performance of the proposed approach.

The rest of the document is organized as follows: Section 2 presents the leak localization methodology in which our work is based. Section 3 describes the problem formulation. Sections 4 and 5 present the sensor placement algorithms proposed in this work, while in Section 6, we show the improvements performed to increase the robustness of the approach. Section 7 shows the application and the results obtained in a real water distribution network. Finally, Section 8 concludes this work.

\section{Leak Location Methodology}

The leak location methodology used in this paper has been introduced in [7,8], as an extension of the methodology proposed in [6]. This approach is summarized here, since it is the basis on top of which the sensor placement algorithm proposed in this paper will be formulated.

The leak location methodology aims to detect and isolate leaks in a DMA using pressure measurements and their estimation using the hydraulic network model. Let us consider a DMA with $m$ demand nodes and $n$ pressure sensors. The leak detection methodology is based on the computation of the residual vector $\mathbf{r}=\left[\begin{array}{lll}r_{1} & \ldots & r_{n}\end{array}\right]^{T}$, where the residual, $r_{i} \in \mathbf{r}$, is the difference between the pressure measurements, $p_{i}$, and its corresponding estimation, $\hat{p}_{i}$, obtained from the simulation of the hydraulic model with no leak, i.e.,:

$$
r_{i}=p_{i}-\hat{p}_{i}
$$

for $i=1, \ldots, n$. Note that there is one residual for each pressure measurement available in DMA.

The leak isolation method relies on analyzing the residual vector (1) using sensitivity analysis, which is determined from the different effects on every pressure measurement caused by each 
possible leak at a time. To perform such sensitivity analysis, the following sensitivity vectors are derived from simulated leak scenarios [6]:

$$
\mathbf{s}_{j}=\left[\begin{array}{c}
\frac{\hat{p}_{1}^{f_{j}}-\hat{p}_{1}}{f_{j}} \\
\vdots \\
\frac{\hat{p}_{n}^{f_{j}}-\hat{p}_{n}}{f_{j}}
\end{array}\right]
$$

for $j=1, \cdots, m$, where $\hat{p}_{i}^{f_{j}}$ and $\hat{p}_{i}$ are the pressure estimation obtained from the hydraulic DMA model simulation under the leak $f_{j}$ scenario and the leak-free scenario, respectively. More precisely, each simulated fault scenario is performed by injecting a leak of a magnitude of $f_{j}$ in the $j^{\text {th }}$ DMA network node in order to compute the sensitivity vector (2). For the sake of simplicity and without loss of generality, $m$ possible leaks (one for each node) have been assumed. Then, the leak isolation is based on the analysis of the residual vector, together with the sensitivity vectors, in order to determine which node has the highest risk of presenting a leakage. A variety of metrics can be used to perform this isolability analysis [15]. In this work, one of the methods presented in [7] based on projections between residual and sensitivity vectors is used. According to the mentioned study, this method presents the best performance for the location task. However, it is important to note that the sensor placement approach proposed in this paper could also be applied using any other leak location method based on sensitivity analysis (e.g., methods based on the other metrics in [7]).

Let $\mathbf{r}$ be the residual vector (1) obtained from the pressure sensors installed in the network. Its normalized projections, $\psi_{j}$, onto each sensitivity vector are computed as:

$$
\psi_{j}=\frac{\mathbf{r}^{T} \mathbf{s}_{j}}{|\mathbf{r}|\left|\mathbf{s}_{j}\right|}
$$

for $j=1, \ldots, m$. Then, the largest projection will determine the candidate node that presents a leak, i.e., a leak in node $k$ is located if:

$$
\psi_{k}=\max \left(\psi_{1}, \cdots, \psi_{m}\right)
$$

\section{Problem Formulation}

The objective of this work is to develop an approach to place a given number of sensors, $n$, in a DMA of a water distribution networks (WDN) in order to obtain a sensor configuration with a maximized leak isolability performance for a given leak detection and isolated scheme. In this work, we use the method based on projections that has been presented in the previous section.

It should be noted that the length of the sensitivity and residual vectors that appear in Equations (1) and (2) corresponds to the number of sensors, $n$, installed in the network. In order to find a sensor configuration that presents maximum isolability performance regarding all the possible leak scenarios, the following residual vectors derived from simulated leak scenarios are computed:

$$
\mathbf{r}_{k}=\left[\begin{array}{c}
\hat{p}_{1}^{f_{k}}-\hat{p}_{1} \\
\vdots \\
\hat{p}_{n}^{f_{k}}-\hat{p}_{n}
\end{array}\right]
$$


for $k=1, \cdots, m$, where $\hat{p}_{i}^{f_{k}}$ and $\hat{p}_{i}$ are the pressure estimation obtained from the hydraulic model simulation under the leak $f_{k}$ scenario and the leak-free scenario, respectively. Note that the magnitude of the leaks used to compute the sensitivity vectors in Equation (2) and the one used to compute the residual vectors in Equation (5) are chosen differently (i.e., $f_{j} \neq f_{k}$ ) in order to increase the robustness of the method. Taking into account the mentioned residual and sensitivity vectors, the sensitivity matrix, $S$, and the residual matrix, $R$, are constructed by concatenating all sensitivities and residuals as follows:

$$
\begin{aligned}
& S=\left[\begin{array}{lll}
\mathbf{s}_{1} & \cdots & \mathbf{s}_{m}
\end{array}\right] \\
& R=\left[\begin{array}{lll}
\mathbf{r}_{1} & \cdots & \mathbf{r}_{m}
\end{array}\right]
\end{aligned}
$$

Note that the matrices, $S$ and $R$, are computed assuming that all the nodes are measured.

To select a configuration with $n$ sensors, the following binary vector is defined:

$$
\mathbf{q}=\left[\begin{array}{lll}
q_{1} & \cdots & q_{m}
\end{array}\right]
$$

where $q_{i}=1$ if the pressure in the node, $i$, is measured, and $q_{i}=0$, otherwise (i.e., the vector $\mathbf{q}$ denotes which sensors are installed). In turn, a diagonal matrix, $Q(\mathbf{q})$, is constructed from the vector, q, as:

$$
Q(\mathbf{q})=\operatorname{diag}\left(q_{1}, \cdots, q_{m}\right)
$$

Then, the corresponding sensitivity and residual vectors can be determined as:

$$
\mathbf{s}_{j}(\mathbf{q})=Q(\mathbf{q}) \mathbf{s}_{j}, \quad \mathbf{r}_{k}(\mathbf{q})=Q(\mathbf{q}) \mathbf{r}_{k}
$$

for $j=1, \cdots, m$, where $\mathbf{s}_{j}$ and $\mathbf{r}_{k}$ are the sensitivity and residual vectors obtained with all nodes measured (i.e., $m=n$, and both vectors, $\mathbf{s}_{j}$, and the vectors, $\mathbf{r}_{k}$, contain $m$ elements each). Finally, the projections in Equation (3) can be computed depending on the sensors with respect to $\mathbf{q}$ as:

$$
\psi_{k j}(\mathbf{q})=\frac{\mathbf{r}_{k}^{T} Q(\mathbf{q}) \mathbf{s}_{j}}{\left|Q(\mathbf{q}) \mathbf{r}_{k}\right|\left|Q(\mathbf{q}) \mathbf{s}_{j}\right|}
$$

for $j=1, \ldots, m$. Note that the property $Q\left(\mathbf{q}^{T}\right) Q(\mathbf{q})=Q(\mathbf{q})$ has been used in Equation (11).

Now, we are able to compute the projection matrix, $\Psi$, as:

$$
\Psi(\mathbf{q})=\left[\begin{array}{ccc}
\psi_{11}(\mathbf{q}) & \cdots & \psi_{1 m}(\mathbf{q}) \\
\vdots & \ddots & \vdots \\
\psi_{m 1}(\mathbf{q}) & \cdots & \psi_{m m}(\mathbf{q})
\end{array}\right]
$$

In order to evaluate the quality of a sensor configuration regarding its capacity to locate a leak at node $i \in\{1, \cdots, m\}$, and assuming the case of a single leak, the next error index is introduced:

$$
\varepsilon_{i}(\mathbf{q})= \begin{cases}0 & \text { if } \psi_{i i}(\mathbf{q})=\max \left(\psi_{i 1}(\mathbf{q}), \ldots, \psi_{i m}(\mathbf{q})\right) \\ 1 & \text { otherwise }\end{cases}
$$

This means that the error index $\varepsilon_{i}=0$, as long as the leak in node $i$ is perfectly located, and $\varepsilon_{i}=1$, otherwise. 
As the objective is to maximize the isolability regarding leaks in all network nodes, the error index that takes into account all the nodes leaks is computed as:

$$
\bar{\varepsilon}(\mathbf{q})=\sum_{i=1}^{m} \frac{\varepsilon_{i}(\mathbf{q})}{m}
$$

We remark that $\bar{\varepsilon}(\mathbf{q})=0$, as long as a sensor configuration is chosen, such that all possible leaks can be perfectly located, and $100 \cdot \bar{\varepsilon}(\mathbf{q})$ is the percentage of incorrectly located leaks.

Based on the vector, $\mathbf{q}$, and the extended error index, $\bar{\varepsilon}(\mathbf{q})$, the sensor placement problem is cast as an optimization problem formulated as:

$$
\begin{aligned}
& \min _{\mathbf{q}} \bar{\varepsilon}(\mathbf{q}) \\
& \text { s.t. } \\
& \sum_{i=1}^{m} \mathbf{q}_{i}=n
\end{aligned}
$$

where $\mathbf{q}$ is defined in Equation (8) and $n \in\{1, \ldots, m\}$ is the number of sensors we want to place.

Remark. It is important to note that the solution of the previous optimization algorithm provides the best sensor location when the size of the leak that we want to locate is close to the value used for evaluating residuals Equation (5). If the leak size is smaller or larger than this value, the optimal sensor location could vary. Moreover, the obtained leak isolation error could be larger than the minimum value obtained as the solution of the optimization problem Equation (15). In a later section, we will see how we propose to introduce some robustness (i.e., against leak magnitude changes), improving the overall sensor placement method.

\section{Semi-Exhaustive Search Approach}

\subsection{Semi-Exhaustive Search}

As stated in Section 3, the problem of sensor placement involves finding an $n$-sensor configuration among a set of $m$ candidate nodes. One trivial approach to solve the problem would be to check all the $\left(\begin{array}{c}m \\ n\end{array}\right)$ sensor configurations. However, this would result in a very high computational cost. Here, we propose the first algorithm as an alternative to this trivial methodology in order to ensure the optimal location in a benchmark network. This method involves the search for the best configuration based on every possible combination, but using lazy evaluation mechanisms to reduce the computation cost by discarding configurations as soon as we see that they cannot be candidates for the optimum. 


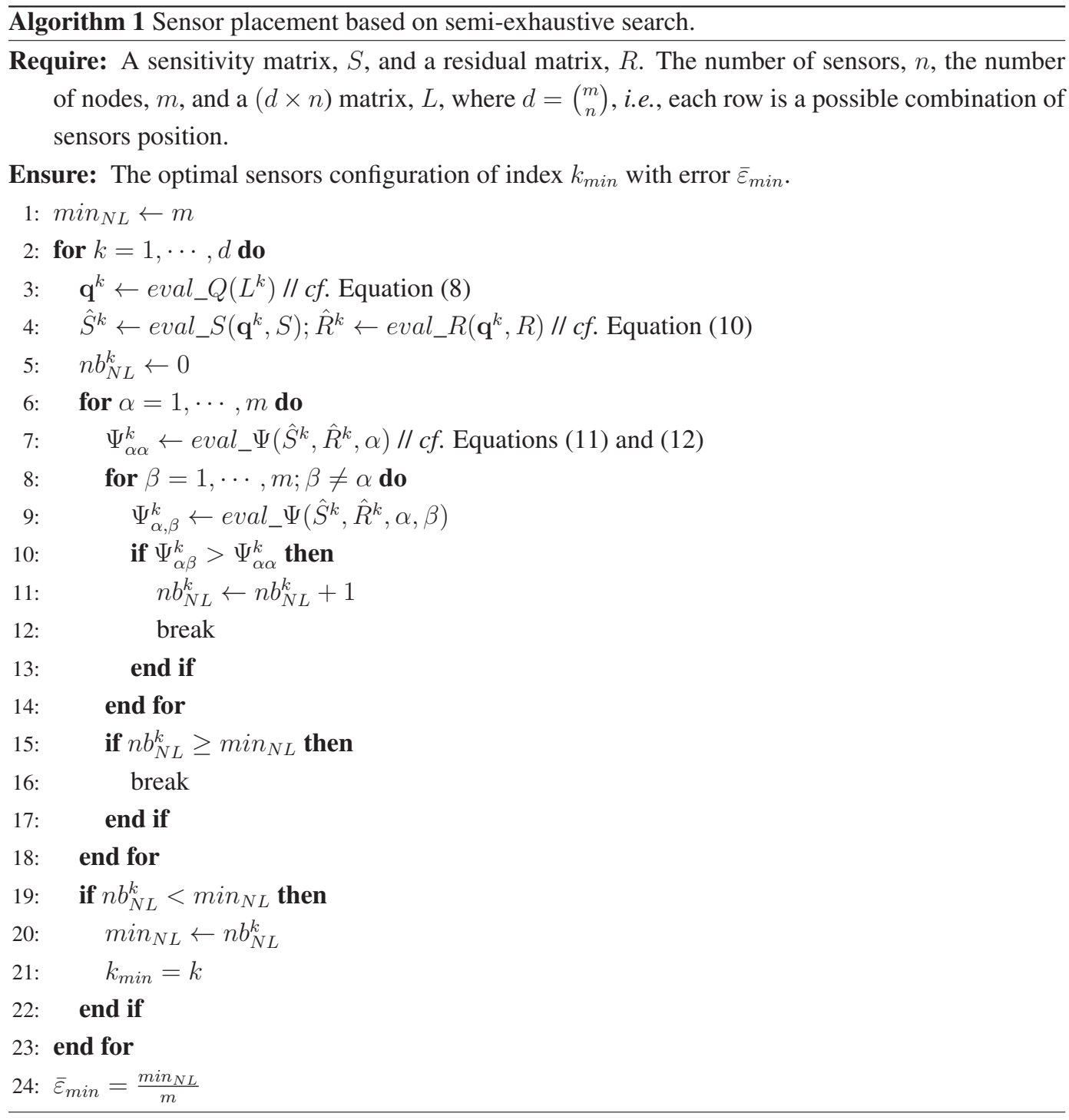

The method is described in Algorithm 1. The goal of this algorithm is to find the optimal sensor configuration, taking into account all the possible combinations of sensors and considering the method that will be used to perform the leak location. First, the algorithm initiates the minimum number of non-localizable (NL) leaks, $\min _{N L}$, found so far to $m$ (line 1). Then, a loop is performed over each possible combination, $k$, of sensor configuration (line 2). The binary vector, $\mathbf{q}^{k}$, is evaluated, which allows one to compute the updated sensitivity and residual matrices, i.e., $\hat{S}^{k}$ and $\hat{R}^{k}$, respectively (lines 3 and 4), and the current number of NL leaks is initiated to be zero (line 5). Then, the algorithm checks for each potential leak, $\alpha$, if it can be located with the current sensor configuration. It evaluates the element $(\alpha, \alpha)$ of the matrix, $\Psi$, and for each other column, $\beta$, of row, $\alpha$, it tests if the projection gives a higher score (line 10). If that is the case, then the number of NL leaks is augmented (line 11), and the other columns of the $\Psi$ matrix do not need to be tested 
(line 12). When the number of NL leaks is higher than the minimum number of NL leaks found so far, i.e., $n b_{N L}^{k} \geq \min _{N L}$, then the current configuration cannot be optimal, and the algorithm aborts the evaluation and continues with the next configuration (line 16), improving, in this way, the computational efficiency of the algorithm. Otherwise, the minimum number of NL leaks is updated by the current number of NL leaks (line 20) and the index of the configuration is taken as the best index found so far (line 21). This algorithm performs a semi-exhaustive search in the sense that all the configurations are considered, but useless computations are avoided as much as possible.

\subsection{Hanoi Network Application Example}

The semi-exhaustive approach was tested in the water network of Hanoi, Vietnam [16]. This benchmark has been used in several works $[17,18]$, where the goal was to design or optimize the operation of a water network. The network consists of 31 demand nodes, one reservoir node and 34 pipes. The first test uses Algorithm 1 to compute the optimal location in the case of two sensors with $\bar{\varepsilon}_{\min }=\min _{N L} / m$. The network model is simulated using EPANET [19]. To study the effect of the leak magnitude on the sensor placement algorithm, the leak magnitude is varied by changing the node emitter coefficient $(E C)$ in EPANET from two to eight (i.e., corresponding to leaks between 20 and 80 liters per second (lps)) and computing the resulting sensitivity and residual matrices, $S$ and $R$ (the $E C$ of each node to be specified for individual leaks is given by $E C=w / p^{p_{e x p}}$ where $w$ is the water flow, $p$ is the fluid pressure and $p_{\exp }$ is a fixed pressure exponent). Note that for this test network, these values are chosen in proportion to the demands of the network, in order to cause a perceptible effect in the pressure. Table 1 presents the leak isolation error index (see Equation (14)) obtained when $S$ and $R$ are computed using different $E C$ values. It can be noticed that even in the worst case, the error index is lower than 0.2 , meaning that less than $20 \%$ of the leaks (i.e., six leaks) are not located in the right node. We can conclude that for a small network, the leak isolation errors, due to the unknown leak size, are small, even when installing just a few sensors. The diagonal elements of Table 1 are not computed, since they correspond to the ideal case where no uncertainties are considered and the minimum error is obviously zero.

Table 1. Minimum error indices in the Hanoi network after placing two sensors. EC, emitter coefficient.

\begin{tabular}{ccccccccc}
\hline & \multicolumn{8}{c}{ EC Used in Residuals } \\
\cline { 2 - 9 } & 2 & 3 & 4 & 5 & 6 & 7 & 8 \\
\hline & 2 & & 0.032 & 0.032 & 0.129 & 0.129 & 0.129 & 0.193 \\
& 3 & 0.032 & & 0.032 & 0.096 & 0.129 & 0.129 & 0.161 \\
& 4 & 0.064 & 0.032 & & 0 & 0.064 & 0.096 & 0.129 \\
EC Used in Sensitivities & 5 & 0.161 & 0.064 & 0.032 & & 0.032 & 0.064 & 0.096 \\
& 6 & 0.161 & 0.129 & 0.064 & 0 & & 0.032 & 0.096 \\
& 7 & 0.193 & 0.161 & 0.129 & 0.064 & 0.032 & & 0 \\
& 8 & 0.193 & 0.193 & 0.161 & 0.129 & 0.064 & 0 & \\
\hline
\end{tabular}


As the second test, we perform the same experiment, but with three sensors. The results are shown in Table 2. First, we note that the error index, due to the unknown leak magnitude, is reduced when more sensors are installed. In both cases (with two and three sensors), the best configuration of sensors used to compute the error index is dependent on the combination of ECs used to compute the matrices, $S$ and $R$. Thus, it is not possible to take a direct decision about the optimal sensor placement with these results. To mitigate this problem, we propose a post-treatment analysis to choose such placement. Since the network is small, when we change the sensitivities and the leak magnitudes, there are some configurations that are repeated many times. We will take advantage of such behavior and select the node configurations with the highest occurrence in the results of the semi-exhaustive search.

Table 2. Minimum error indices in Hanoi network with three sensors.

\begin{tabular}{cccccccccc}
\hline & \multicolumn{8}{c}{ EC Used in Residuals } \\
\cline { 2 - 9 } & 2 & 3 & 4 & 5 & 6 & 7 & 8 \\
\hline & 2 & & 0 & 0 & 0.032 & 0 & 0.032 & 0.032 \\
& 3 & 0 & & 0 & 0 & 0.032 & 0 & 0.032 \\
& 4 & 0 & 0 & & 0 & 0 & 0.032 & 0.032 \\
EC Used in Sensitivities & 5 & 0.032 & 0 & 0 & & 0 & 0.032 & 0.032 \\
& 6 & 0 & 0 & 0 & 0 & & 0 & 0.032 \\
& 7 & 0.032 & 0.032 & 0.032 & 0 & 0 & & 0 \\
& 8 & 0.032 & 0.032 & 0.032 & 0 & 0 & 0 & \\
\hline
\end{tabular}

In order to choose an adequate combination of sensors, we count the occurrences of the configurations leading to the error indices in Tables 1 and 2 and look for those that are found the most. The idea is to find those configurations with the minimal error index that cover as many different leak magnitudes as possible. In the example of the Hanoi network, the three configurations with the highest occurrence in the case of the placement of two sensors are:

- Nodes $\{12,21\}$ with 16 occurrences

- Nodes $\{12,13\}$ with 13 occurrences

- Nodes $\{7,12\}$ with seven occurrences

and in the case of three sensors, the three configurations with highest occurrence are:

- Nodes $\{12,14,21\}$ with 22 occurrences

- Nodes $\{12,21,27\}$ with 22 occurrences

- Nodes $\{12,21,29\}$ with 18 occurrences

Table 3 gives the error indices averaged over each combination of residuals and sensitivities for the three best configurations, in the case of two and three sensors. Among these candidates, we consider the one that leads to the lowest error. It appears that in the case of two sensors, the optimal sensor configuration for the Hanoi network corresponds to the pair of nodes $\{12,21\}$, whereas 
in the case of three sensors, the best configuration is obtained installing the sensors at the nodes $\{12,14,21\}$.

Table 3. Best configurations and corresponding error indices for different leak magnitudes in the Hanoi network.

\begin{tabular}{cccc}
\hline Two Placed Sensors & $(n=2)$ Case & Three Placed Sensors $(n=3)$ Case \\
\hline Configuration & $\bar{\varepsilon}(\mathbf{q})$ & Configuration & $\bar{\varepsilon}(\mathbf{q})$ \\
$\{12,21\}$ & 0.131 & $\{12,14,21\}$ & 0.025 \\
$\{12,13\}$ & 0.133 & $\{12,21,27\}$ & 0.028 \\
$\{7,12\}$ & 0.157 & $\{12,21,29\}$ & 0.035 \\
\hline
\end{tabular}

\section{Genetic Algorithms Approach}

\subsection{Introduction}

Genetic algorithms (GAs) are well-known search and optimization tools based on the principles of natural genetics and natural selection [20,21]. Because of their broad applicability, ease of use and global perspective, GAs have been increasingly applied to various search and optimization problems in the recent past. Some fundamental ideas of genetics are borrowed and used artificially to construct search algorithms that are robust and require minimal problem information. GAs transform a population of individual objects, each with an associated fitness value, into a new generation of the population using the Darwinian principle of reproduction and survival of the fittest and analogs of naturally occurring genetic operations, such as crossover (sexual recombination) and mutation. Each individual in the population represents a possible solution to a given problem. The genetic algorithm attempts to find a very good (or the best) solution to the problem by genetically breeding the population of individuals over a series of generations.

The GAs can be used in the context of sensor placement in WDN in order to find near-optimal placement for leak location. In that case, a chromosome corresponds to the possible presence or absence of a sensor at a given node.

\subsection{Algorithm Description}

Here, the sensor placement problem formulated as an optimization problem in Section 3 is solved using genetic algorithms and implemented using the GA Toolbox of MATLAB. The GA needs to establish a function whose output involves an index to be minimized. In our case, this function corresponds to the evaluation of the error index computed in Equation (14) according to the computation of the projection matrix, as in Equation (12). This error depends on the number of maximum values in each row of the matrix that are not elements of the diagonal in the projection matrix. 


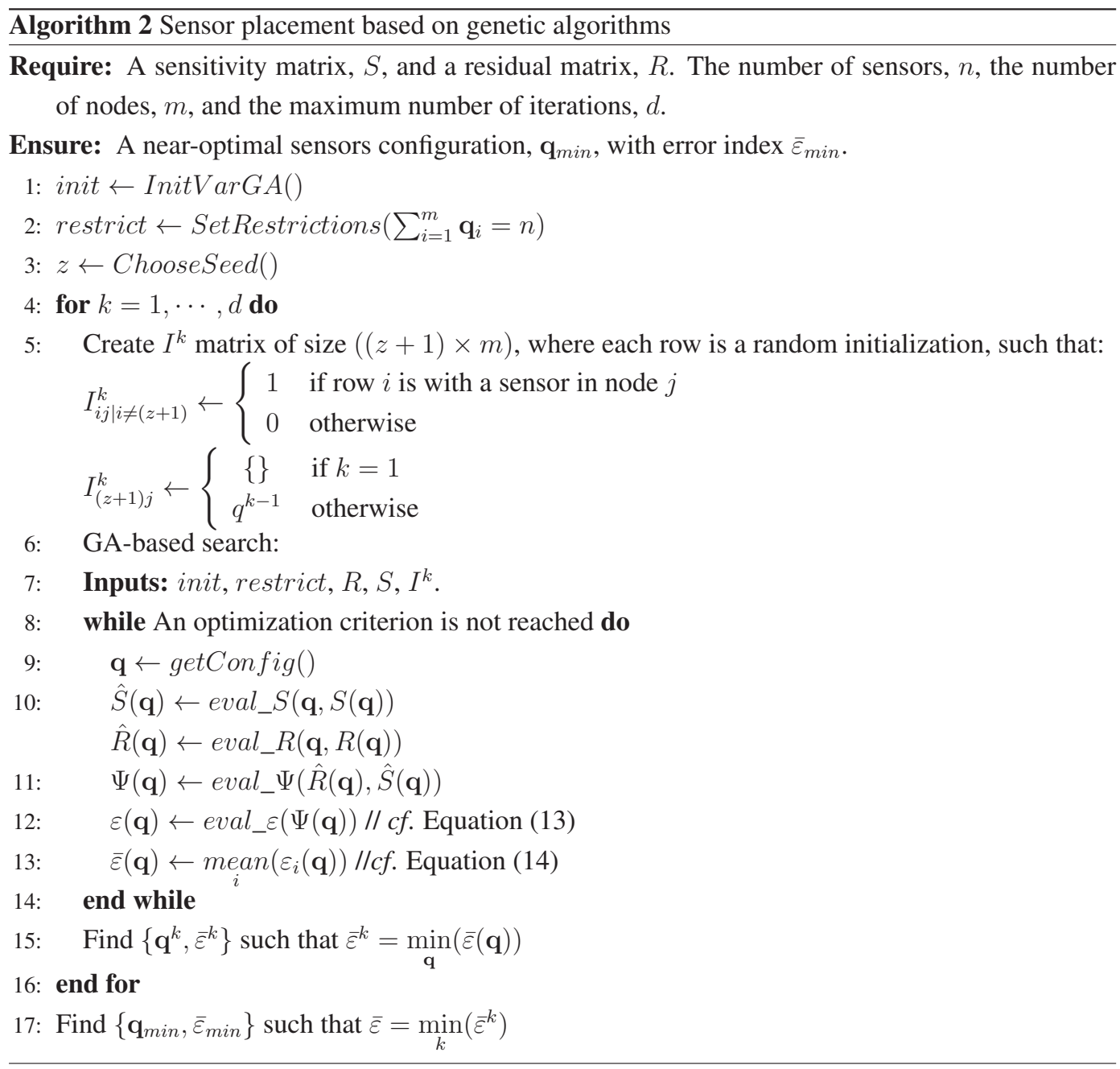

The pseudo-code of the algorithm is shown in Algorithm 2. First, we initialize the variables of the GA (line 1), including the number of generations, the bit string type population, the tolerance as $10^{-10}$ and the elite count as one, in order to save one of the previous results analyzed. Then, we declare the search restriction (line 2), being that the number of "ones" in the solution corresponds to the number of sensors to install, and a seed size, $z$, is chosen (line 3). This seed creates an initial matrix with random sensor positions, and every location delivered by the GA is tested according to the function declared in the algorithm. The sensor placement is based on the construction of binary vectors, where the presence of a "one" represents a sensor located in the correspondent node. This vector allows one to select the adequate rows of the matrices, $S$ and $R$, in order to compute the projection matrix according to the selected nodes to be measured. Once we have this projection matrix, we look for the maximum value of each row of the matrix, expecting the highest to be in the diagonal position. If it occurs, it means that the leak index equal to the row in question can be located with the selected sensor configuration. Otherwise, the leak cannot be located using this configuration. 


\subsubsection{Hanoi Network Application Example}

The experiments performed in the Hanoi network presented in Section 4.2 and based on a semi-exhaustive search were computationally very demanding, despite the lazy evaluation mechanisms involved. For such a simple example, 465 configurations had to be tested for the case of two sensors with an average computation time of $3 \mathrm{~s}$ and 4,495 configurations for the case of three sensors, which takes $15 \mathrm{~s}$ on average. This means that performing a semi-exhaustive search in a large network is not feasible, because of the computational complexity that would quickly lead to testing millions of possible combinations. This is the motivation for using GA. All the tests performed using semi-exhaustive search are reproduced using Algorithm 2 applied to the Hanoi network. The solutions found are the same as the ones obtained with the semi-exhaustive search, but with a computational time lower than 9 s per iteration and including three generations in each of them. All the experiments were performed in MATLAB, using a Windows 7 computer with a Pentium Dual Core processor of $2 \mathrm{GHz}$, memory (RAM) of $4 \mathrm{~GB}$ and a 64-bit operating system.

\section{Robust Sensor Placement}

\subsection{Improving the Sensor Placement Robustness}

In our experiments, despite Algorithm 2 providing efficiently optimal solutions (since they are consistent with the semi-exhaustive search), we have seen that the algorithm requires a post-treatment analysis in order to make an adequate sensor placement decision when uncertainty (e.g., about the unknown leak magnitude) is considered. Moreover, we know that this placement represents a near-optimal solution that works only for the time instant evaluated. In the following, we present three improvements that avoid any post-treatment and increase the robustness of the GA-based sensor placement.

\subsubsection{Time Horizon Analysis}

In the semi-exhaustive search and GA-based algorithm that we have presented, we took into account a single instant of time for the analysis. However, the configuration that gives the minimum error index in the leak isolation can vary when the demand changes along a given period of time. To address this problem, it is possible to improve the tasks of leak detection and isolation by considering a time horizon, as suggested in [7]. Thus, in the following, we incorporate a time horizon in the evaluation function of the GA, with the objective of increasing the quality of the sensor placement for a better leak isolation within the network. Note that there is no restriction on the time horizon magnitude.

The GA evaluation function is modified in order to work with the mean projection along the time horizon instead of using a single instant of time. With such modification, the candidate leak node is obtained by looking at the maximum of the mean projection, $\bar{\Psi}(\mathbf{q})$, defined by: 


$$
\bar{\Psi}(\mathbf{q})=\frac{1}{T} \sum_{t=1}^{t=T} \Psi^{t}(\mathbf{q})
$$

where $\Psi^{t}(\mathbf{q})$ is computed using Equation (12) and $T$ represents the number of time samples associated with the time horizon.

\subsubsection{Distance-Based Scoring}

In the optimization problem Equation (15), the error index function Equation (13) was set to zero each time a leak was located in the correct node and to one, otherwise. This binary scoring process treats all the leak nodes that are incorrectly located in the same way. However, it may be interesting to provide a more informative scoring that would consider if the location returned is close to the real leak position or not.

We propose to rely on topological distances (i.e., the number of nodes in the shortest path from one node to another in the network) with respect to the real leak position for evaluate the scores. When the topological distance increases, we linearly increase the error scoring until a cut-off distance, $d_{\text {max }}$, for which the node score is then set to one. Thus, Equation (13) is replaced by the scoring function:

$$
\varepsilon_{i}(\mathbf{q})=\left\{\begin{array}{cl}
\frac{d_{i}(\mathbf{q})}{d_{\max }} & \text { if } d_{i}(\mathbf{q})<d_{\max } \\
1 & \text { otherwise }
\end{array}\right.
$$

where $d_{i}$ represents the topological distance between the leak node, $i$, and the node, $j$, that corresponds to the biggest projection value, i.e., $\psi_{i j}(\mathbf{q})=\max \left(\psi_{i 1}(\mathbf{q}), \ldots, \psi_{i m}(\mathbf{q})\right)$.

The cut-off distance, $d_{\max }$, can be chosen by the user. In our case, we propose a distance that depends on the network size. Let us consider a network with the shape of a 2D squared-grid made of $m=(2 i+1)^{2}$ nodes. Then, the distance from the center node to the network border would be given by $\frac{1}{2} \sqrt{m}$. Following such a relationship between topological distance and number of nodes, we propose to set the cut-off distance, such that:

$$
d_{\max }=\frac{1}{2} \sqrt{m}
$$

\subsubsection{Robustness Regarding Leak Magnitude Variations}

The choice of sensor placement is affected by the leak magnitude taken to build the sensitivities. However, in real scenarios, this magnitude cannot be determined in advance. To improve the robustness of the results according to such parameter changes, we propose to incorporate sets of sensitivities and residuals in the evaluation function that are computed from different leak magnitudes. Assume that there are $L$ leak magnitudes $l \in\{1, \cdots, L\}$, each one associated with a residual matrix, $R^{l}$, and a sensitivity matrix, $S^{l}$. Then, the number of possible couples, $\left\{R^{l_{1}}, S^{l_{2}}\right\}$, with $l_{1}, l_{2} \in\{1, \cdots, L\}$ would be $L^{2}$. Among them, we discard couples $\left\{R^{l}, S^{l}\right\}$ built from the same leak magnitude, since in realistic scenarios, the leak magnitude used to build the sensitivity matrix will not match the real leak magnitude from which is built the residual matrix. Furthermore, we discard couples $\left\{R^{l_{1}}, S^{l_{2}}\right\}$ with $l_{1}>l_{2}$, since $\Psi\left(R^{l_{1}}, S^{l_{2}}\right)=\Psi\left(R^{l_{2}}, S^{l_{1}}\right)^{T}$, and thus, they would 
lead to the same error index. Therefore, we obtain a total of $\left(\begin{array}{c}L \\ 2\end{array}\right)$ couples of residual and sensitivity matrices $\{R, S\}$. Finally, the error index that we computed in Equation (14) will be now evaluated, taking into account the average of the error indices computed for each of these couples:

$$
\bar{\varepsilon}(\mathbf{q})=\frac{1}{m\left(\begin{array}{l}
L \\
2
\end{array}\right)} \sum_{i=1}^{m} \sum_{c=1}^{\left(\begin{array}{c}
L \\
2
\end{array}\right)} \varepsilon_{i}^{c}(\mathbf{q})
$$

\subsection{Robust Sensor Placement Algorithm}

The three steps presented above increase the robustness of the sensor placement method with respect to the experimental variations. These new procedures shown in Algorithm 3 modify the iteration steps performed by GA that were previously presented in Algorithm 2.

The method basically consists of $d$ iterations of the GA. At each step, it starts with an initialization phase of the GA similar to the one performed in Algorithm 2 (line 1). Then, iterations within the GA search are performed until one of the GA stop criteria is reached (line 5). To build the evaluation function, a loop is executed over each possible combination of input residual and sensitivity matrices, according to the different leak magnitudes (line 7). For each combination, $c$, and for each time instant, $t$ (line 8 ), the updated residual and sensitivity matrices, as well as the projection matrices are evaluated (line 9 to 11 ). Then, we compute $\Psi^{c}(\mathbf{q})$, i.e., the mean of the projection matrix over each time instant (line 13) and the resulting error vector, $\epsilon^{c}(\mathbf{q})$ (line 14). Next, the averaged error index is evaluated (line 16), and the GA searches for the configuration, $\mathbf{q}^{k}$, with minimum error index $\bar{\varepsilon}^{k}$ (line 18). Finally, we look among the $d$ configurations returned by the GA for the one with the best score (line 20).

\subsection{Application to the Hanoi Network}

Algorithm 3 was applied to the Hanoi network. In our tests, we take $L=7$ leak magnitudes (20, 30, 40, 50, 60, 70 and 80 liters per second), which results in 21 couples of residual and sensitivity matrices. Furthermore, we use a 24-h time horizon with pressure measurements every hour, which gives a total of $T=25$ time steps. The algorithm performs five main iterations with two generations in each of them, while the seed size is made of 30 vectors in the initial population.

Since the network has 31 nodes, Equation (18) returns a cut-off distance of three nodes to compute the scoring error in Equation (17). Thus, leak nodes with topological distances of zero, one and two nodes have an error of zero, $\frac{1}{3}$ and $\frac{2}{3}$, respectively. Nodes with a higher distance have an error of one.

Algorithm 3 is executed varying the number of sensors from two to ten. Figure 1 shows the scoring error according to the number of sensors. We can see that after three sensors, the reduction of the error strongly decreases, and the use of additional sensors is not necessarily justified. This suggests that three sensors would be a good choice to have reliable leak detection and location. Furthermore, the best placement corresponds to nodes $\{12,21\}$ with an error index of 0.061 in the case of two sensors, and it corresponds to nodes $\{12,14,21\}$ with an error of 0.011 in the case of three sensors. Note that since this is a small example, these results give the same configurations as the ones found with both the semi-exhaustive search and Algorithm 2. 


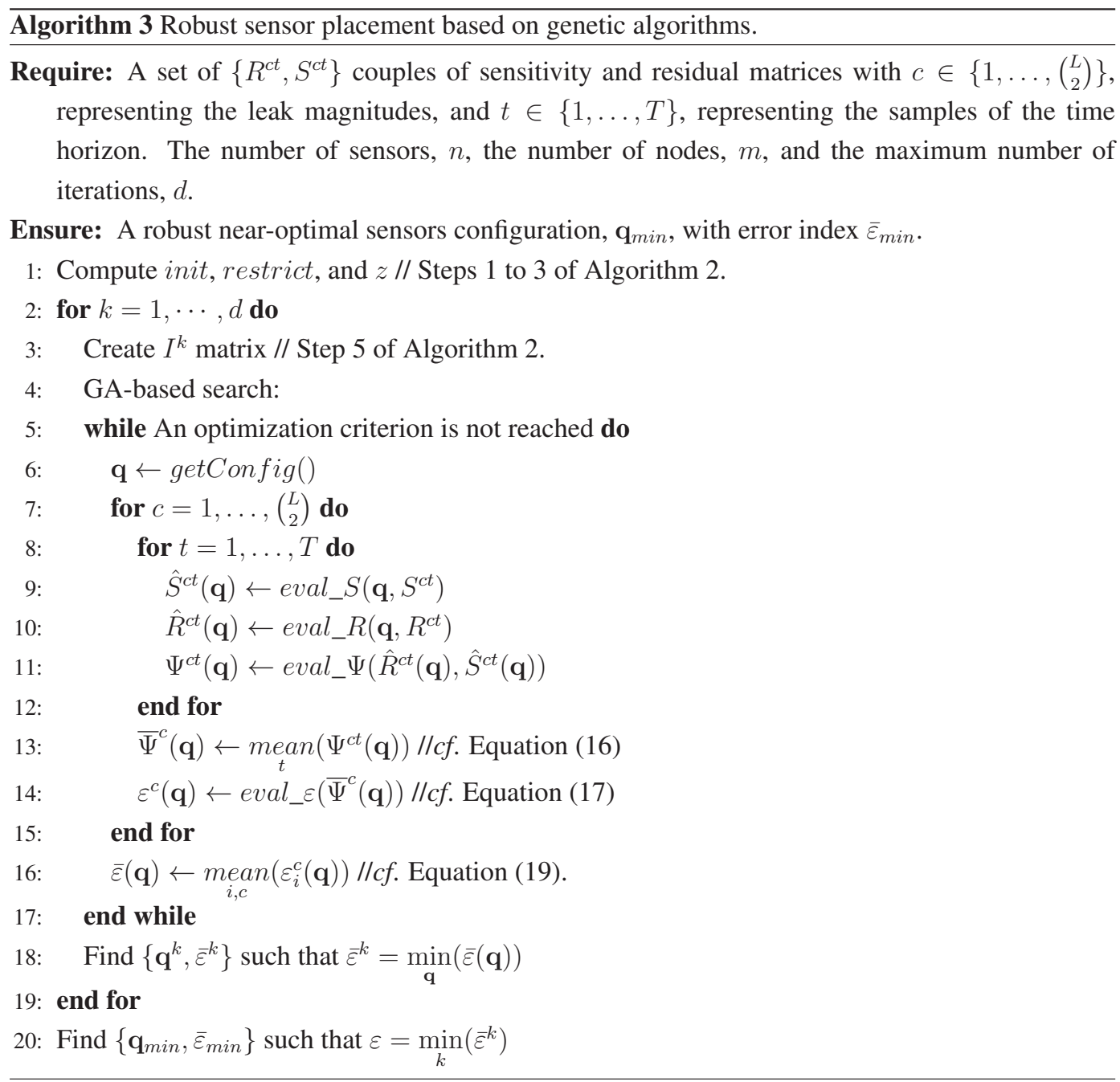

\section{Case Study: Limassol Network}

The methodologies presented in Algorithms 2 and 3 are applied to a real network. We used the Limassol network in Cyprus that has a total of 197 demand nodes and is represented in Figure 2. The network model is available in EPANET, as was the case for the Hanoi network. First, the semi-exhaustive algorithm is used to obtain three sensor placements that will serve as a reference to evaluate the performance of the GA approach. This algorithm is time-demanding in this case, since there are more than $1.2 \times 10^{6}$ possible combinations of nodes to be considered. The computation time consumed by the semi-exhaustive search was approximately $60 \mathrm{~h}$ for the combination of sensitivity and residual chosen. This means that testing all the possible combinations of sensitivities and residuals is not feasible. The sensor placement problem is set up with $E C=0.25$ (a leak of approximately $1.67 \mathrm{lps}$ ) for the sensitivities and $E C=0.20$ (leak of approximately $1.3 \mathrm{lps}$ ) for the residuals. The best configuration obtained leads to placing sensors in nodes $\{82,133,157\}$, which gives an error index $\bar{\varepsilon}_{\text {min }}=0.258$. 
Figure 1. Minimum error index according to the number of sensors in the Hanoi network.

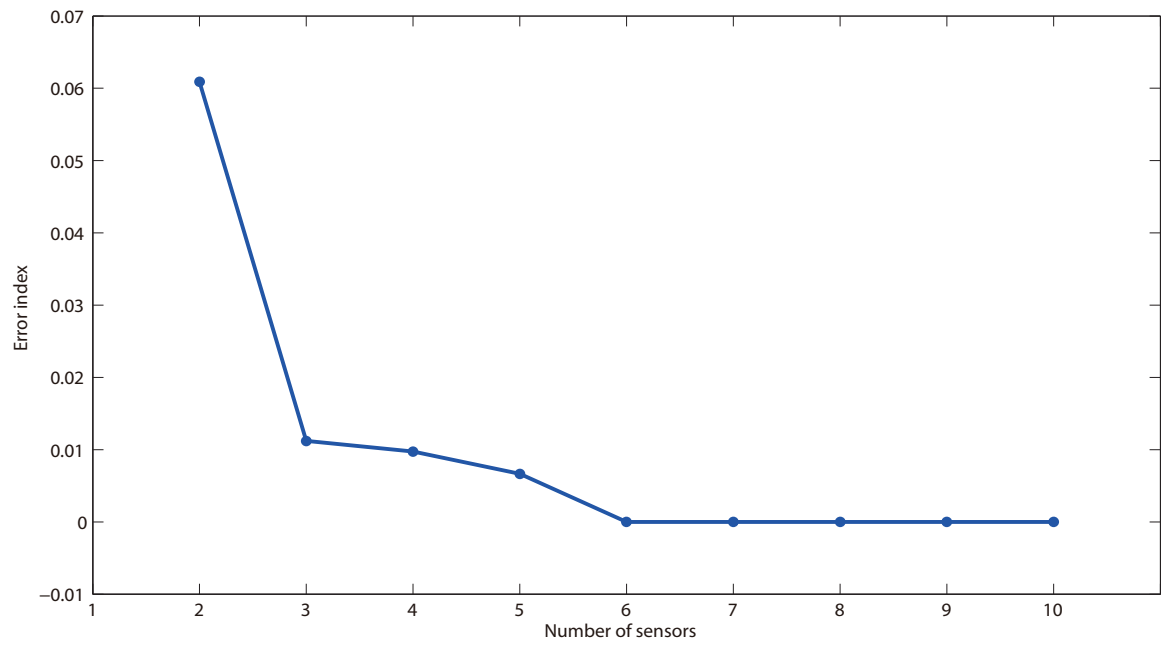

Figure 2. Water network in Limassol, Cyprus.

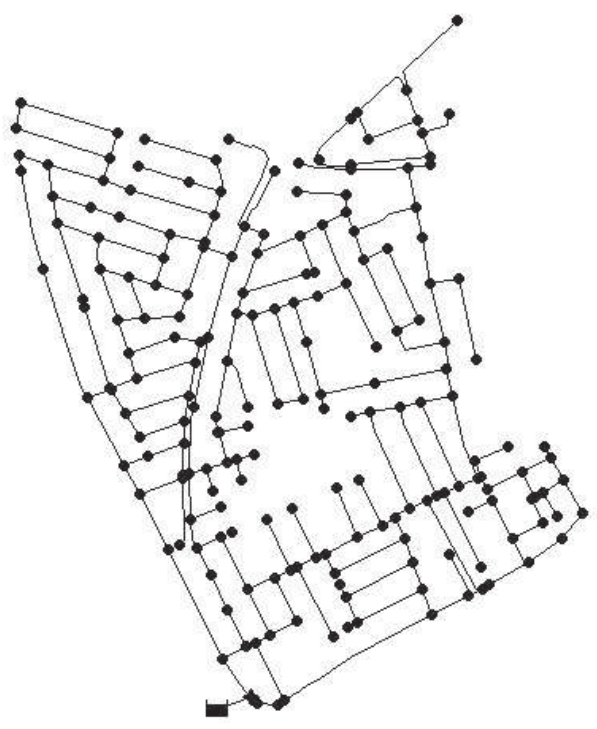

\subsection{Application of the Sensor Placement Based on Genetic Algorithms}

We apply Algorithm 2 for different types of residual and sensitivity matrices that are computed by varying the leak magnitudes within a given range. Here, the robustness improvements presented in Section 6.1 are not applied (they will be applied in the next subsection). The parameters for the genetic algorithm were selected after several trial and error tests. The initialization matrix 
was set with a size of 50 rows, and five iterations were allowed in order to increase the efficiency of the method with a maximum of five generations in each of them. The computation time was about $60 \mathrm{~min}$. Compared to the time spent in the semi-exhaustive search, we can conclude that GA significantly reduces the time required to find a solution. Table 4 shows the nodes for each combination of EC value, whereas Table 5 shows the corresponding minimum error indices. From these tables, we can see that the algorithm finds different configurations depending on the leak magnitude (through EC) selected for the sensitivities and for the residuals. Such behavior was also occurring with the Hanoi network ( $c f .4 .2)$.

Table 4. Sensor configurations in Limassol network with three sensors.

\begin{tabular}{|c|c|c|c|c|c|c|}
\hline & & \multicolumn{5}{|c|}{ Residuals EC } \\
\hline & & 0.15 & 0.2 & 0.25 & 0.3 & 0.35 \\
\hline \multirow{5}{*}{ Sensitivities EC } & 0.15 & & $\{40,77172\}$ & $\{25,77,133\}$ & $\{76,133,185\}$ & $\{76,133,152\}$ \\
\hline & 0.2 & $\{76,133,152\}$ & & $\{76,86,152\}$ & $\{77,124,152\}$ & $\{76,110,173\}$ \\
\hline & 0.25 & $\{85,156,196\}$ & $\{8,76,150\}$ & & $\{75,116,157\}$ & $\{72,115,150\}$ \\
\hline & 0.3 & $\{72,118,163\}$ & $\{76,133,141\}$ & $\{77,111,150\}$ & & $\{75,23,152\}$ \\
\hline & 0.35 & $\{76,128,140\}$ & $\{75,120,150\}$ & $\{77,115,137\}$ & $\{29,112,152\}$ & \\
\hline
\end{tabular}

Table 5. Minimum error indices in the Limassol network for the configurations of Table 4.

\begin{tabular}{ccccccc}
\hline & & \multicolumn{5}{c}{ Residuals EC } \\
\cline { 3 - 7 } & & 0.15 & 0.2 & 0.25 & 0.3 & 0.35 \\
\hline \multirow{3}{*}{ Sensitivities EC } & 0.15 & & 0.324 & 0.294 & 0.299 & 0.314 \\
& 0.2 & 0.299 & & 0.284 & 0.279 & 0.294 \\
& 0.3 & 0.279 & 0.274 & & 0.243 & 0.243 \\
& 0.35 & 0.324 & 0.279 & 0.263 & 0.258 & \\
\hline
\end{tabular}

Thus, we perform a post-treatment analysis to decide what is the best sensor configuration for the network. This is based on the following tests:

- Variation in the tested leak magnitude: We compute the projection matrices for all the configurations found, taking into account the various combination of sensitivity and residual matrices corresponding to leak magnitudes changes.

- Consideration of the sensors precision: To take into account the limitation of the sensor precision, we truncate the two last decimals of the pressure measurements to compute the residual matrices.

- Application of random noise in the measurements: We include Gaussian white noise in the measurements with a mean amplitude corresponding to approximately $0.5 \%$ of the expected measurement, considering the technology of the pressure sensors used by the water company managing the network. 
In order to select the adequate configuration of sensors, we propose performing the experiments described above and look for the combination with the smallest average error index along all the possible leak magnitudes and sensitivities to test. This criterion is analytically established by taking the minimum of the average error indices:

$$
\min \left(\frac{1}{L^{2}} \sum_{j=1}^{L} \sum_{i=1}^{L} \varepsilon^{i j}\right)
$$

where $L$ is the number of leak magnitudes used and $\varepsilon^{i j}$ is the error index (cf. Equations (13) and (14)) obtained with the residual and sensitivity of the respective indices, $i$ and $j$. In this way, the search for the best sensor placement is built as a min-max optimization problem.

Table 6. Averaged error indices for configurations of Table 4.

\begin{tabular}{cccccc}
\hline & & \multicolumn{3}{c}{ Test } \\
\hline \multicolumn{2}{c}{ Configuration } & Magnitude Change & Sensor Precision & Noise Addition & Total Average \\
\hline 1 & $\{75,116,157\}$ & 0.336 & 0.412 & 0.576 & 0.442 \\
2 & $\{85,156,196\}$ & 0.362 & 0.455 & 0.597 & 0.471 \\
3 & $\{72,115,150\}$ & 0.345 & 0.429 & 0.583 & 0.452 \\
4 & $\{76,110,173\}$ & 0.340 & 0.409 & 0.556 & 0.435 \\
5 & $\{77,124,152\}$ & 0.348 & 0.444 & 0.581 & 0.457 \\
6 & $\{76,133,152\}$ & 0.318 & 0.403 & 0.558 & 0.426 \\
7 & $\{76,86,152\}$ & 0.335 & 0.421 & 0.572 & 0.443 \\
8 & $\{25,77,133\}$ & 0.336 & 0.420 & 0.569 & 0.441 \\
9 & $\{76,133,185\}$ & 0.334 & 0.420 & 0.564 & 0.439 \\
10 & $\{40,77,172\}$ & 0.368 & 0.448 & 0.613 & 0.477 \\
11 & $\{\mathbf{7 6 , 1 3 3 , 1 5 2}\}$ & $\mathbf{0 . 3 1 8}$ & $\mathbf{0 . 4 0 3}$ & $\mathbf{0 . 5 4 6}$ & $\mathbf{0 . 4 2 2}$ \\
12 & $\{8,76,150\}$ & 0.356 & 0.462 & 0.613 & 0.477 \\
13 & $\{72,118,163\}$ & 0.373 & 0.443 & 0.576 & 0.464 \\
14 & $\{76,133,141\}$ & 0.341 & 0.416 & 0.570 & 0.442 \\
15 & $\{76,128,140\}$ & 0.355 & 0.425 & 0.573 & 0.451 \\
16 & $\{75,120,150\}$ & 0.328 & 0.431 & 0.582 & 0.447 \\
17 & $\{77,111,150\}$ & 0.342 & 0.422 & 0.566 & 0.443 \\
18 & $\{77,115,137\}$ & 0.330 & 0.417 & 0.561 & 0.436 \\
19 & $\{75,123,152\}$ & 0.339 & 0.421 & 0.575 & 0.445 \\
20 & $\{29,112,152\}$ & 0.394 & 0.455 & 0.590 & 0.480 \\
\hline
\end{tabular}

Table 6 shows the errors induced by the three tests previously mentioned, as well as the total average error. From this table, we choose as the best sensor placement configuration the one with the lowest total average error.

Such a method gives the sensor placement at nodes $\{76,133,152\}$, which provides the lowest average error among the sets of sensor placements computed from the different combinations of residual and sensitivities. However, there is no guarantee that other sets would not lead to better results. Furthermore, this configuration was found based on a single time instant; thus, it is not robust 
to changes in the demand that would occur when considering a period of time. For these reasons and to get a more reliable solution, we prefer the method that includes the robustness improvements that we proposed in Section 6.1.

\subsection{Application of the Robust Sensor Placement Method}

We applied Algorithm 3 to the same Limassol, Cyprus network. We took $L=5$ different leak magnitudes (one, 1.33,1.66, two and $2.33 \mathrm{lps}$ ) that lead to 10 couples of sensitivity and residual matrices. The network data were analyzed taking into account a $T=24 \mathrm{~h}$ time horizon with pressure measurements every hour. Furthermore, the sensor noise (approximately $0.5 \%$ of the expected measurement as indicated above) was added to the computation of the residuals in order to increase the robustness of the method.

Since the network has 197 nodes, Equation (18) returns a cut-off distance of seven nodes to compute the scoring error in Equation (17). The algorithm performs five main iterations with two generations in each of them, while the seed size is made of 50 vectors in the initial population.

After the iterations, the best result obtained corresponds to a sensor configuration of nodes $\{2,75,158\}$, with an error index of 0.302 , which means an average distance of two nodes between the located node and that with the real leak. This placement is shown in Figure 3a.

Figure 3. Near-optimal placement of three sensors in the Limassol network. (a) Sensor placement with noise of $0.5 \%$; (b) sensor placement with noise of $2 \%$.

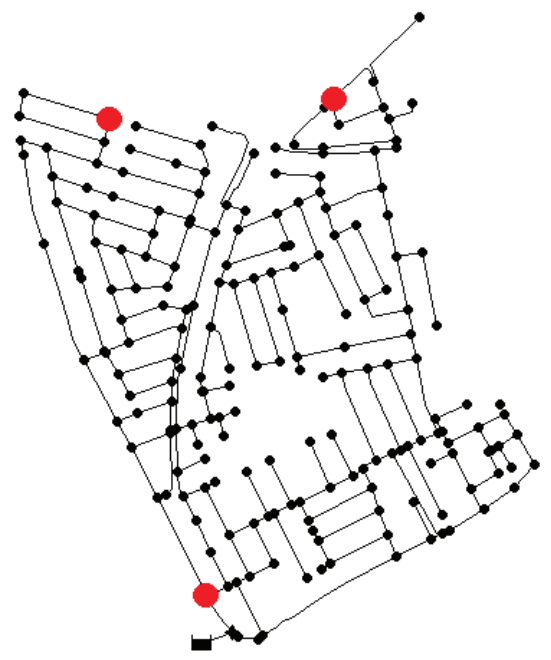

(a)

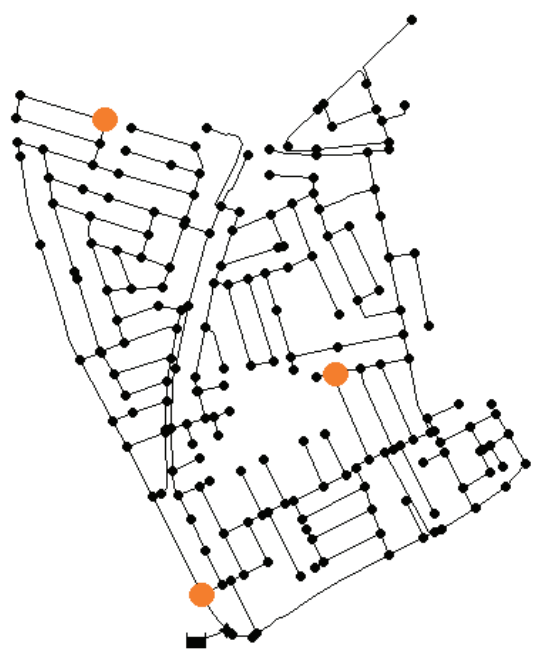

(b)

A second test increasing the noise level up to $2 \%$ gives the best configuration at nodes $\{2,75,100\}$ (Figure 3b) with an error index of 0.712 . This means that leaks are located with an average of five nodes of topological distance to the real leak. Note that two of the nodes are repeated regarding the 
previous location. Consequently, despite the increased level of noise, the placement of the sensors has not been severally affected. Thus, it reassures the fact that the near-optimal solution will have a satisfactory behavior, even in different conditions.

It is important to note that these results comes from the integration within the GA of all the improvements of sensor placement robustness that we described in Section 6.1. Thus, contrary to what was done in the previous section when using Algorithm 2, there is no need to perform any post-treatment analysis to extract a robust solution. Integrating a time horizon, a more informative distance-based scoring and the possible variations of leak magnitude, our method provides a solution configuration for sensor placement with a higher level of confidence.

\subsection{Practical Considerations}

When moving the proposed sensor placement approach to the real network, the following practical considerations should be taken into account considering the previous experience in [6]:

- the DMA EPANET model should be recalibrated with real data obtained from the available sensors already installed in the network (typically at the flow entrance points) in order to minimize the errors due to model mismatch between the real and simulated network.

- the configuration of the internal valves should also be verified in order to assure that their positions in the real and simulated network are the same.

- the nodal demand should be estimated as well as possible using information from water consumption and tele-measurement devices, if available.

- leak size range that is considered, as well as sensor noise and precision should be characterized so that the robust sensor placement approach presented in Section 6 could be used in such a way that the installed sensors guarantee the minimal isolation error that is possible.

\section{Conclusions}

In this paper, we proposed a new approach to sensor placement for water distribution networks that maximizes leak isolability. The sensor placement problem has been formulated as a non-linear integer optimization problem. The optimization criterion is based on minimizing the number of non-isolable leaks according to the isolability criteria introduced. This approach is combined with a projection-based leak location scheme, but it could be easily adapted to any other sensitivity-based isolation scheme.

The first semi-exhaustive search method has been proposed that searches for the best configuration and relies on lazy evaluation mechanisms to reduce the computation cost. However, the computational effort remains too demanding for most realistic scenarios. Thus, we proposed to solve the optimization problem with GAs, which are known to work well in large-sized problems of a non-linear integer nature. We have seen that such approach allows us to find near-optimal solutions in an efficient way. We also highlighted that leak magnitude changes were impacting the resulting best sensor placement found by the GA algorithm, requiring a post-treatment analysis to tackle such a problem. Finally, we proposed three improvements that avoid any post-treatment and increase the 
robustness of the GA-based sensor placement. Experiments on two types of networks were performed to compare the different methods proposed in this paper. They demonstrate the relevance of the robust GA-based approach.

For future work, we would like to combine our robust GA-based approach with other methods to perform the leak isolation task. Furthermore, other types of optimization methods that provide some guarantee regarding the solution optimality could be investigated in the future. Enhancing the robustness of the sensor placement algorithm against additional sources of uncertainty, as in the model parameters or in nodal demand, will also be considered in future research.

\section{Acknowledgments}

This work is supported by the Research Chair in Supervision and Advanced Control of Tecnológico de Monterrey, Campus Monterrey and by a CONACYT studentship. This work has been partially grant-funded by CICYT SHERECS DPI -2011-26243 and CICYT WATMAN DPI-2009-13744 of the Spanish Ministry of Education, by iSense grant FP7-ICT-2009-6-270428 and by EFFINET grant FP7-ICT-2012-318556 of the European Commission. This work is also partially founded by the Fond National de la Recherche, Luxembourg (CO11/IS/1206050).

\section{Conflicts of Interest}

The authors declare no conflict of interest.

\section{References}

1. Puust, R.; Kapelan, Z.; Savic, D.A.; Koppel, T. A review of methods for leakage management in pipe networks. Urban Water J. 2010, 7, 25-45.

2. Khulief, Y.; Khalifa, A.; Mansour, R.; Habib, M. Acoustic detection of leaks in water pipelines using measurements inside pipe. J. Pipeline Syst. Eng. Pract. 2012, 3, 47-54.

3. Lambert, M.; Simpson, A.; Vítkovský, J.; Wang, X.J.; Lee, P. A Review of Leading-Edge Leak Detection Techniques for Water Distribution Systems. In Proceedings of the 20th AWA Convention, Perth, Australia, 6-10 April, 2003.

4. Andrew, F.; Colombo, P.L.; Karney, B.W. A selective literature review of transient-based leak detection methods. J. Hydro-Environ. Res. 2009, 2, 212-227.

5. Pudar, R.S.; Liggett, J.A. Leaks in pipe networks. J. Hydraul. Eng. 1992, 118, 1031-1046.

6. Pérez, R.; Puig, V.; Pascual, J.; Quevedo, J.; Landeros, E.; Peralta, A. Methodology for leakage isolation using pressure sensitivity analysis in water distribution networks. Control Eng. Pract. 2011, 19, 1157-1167.

7. Casillas, M.V.; Garza-Castañón, L.; Puig, V. Extended-Horizon Analysis of Pressure Sensitivities for Leak Detection in Water Distribution Networks. In Proceedings of the 8th IFAC Symposium on Fault Detection, Supervision and Safety of Technical Processes, Mexico city, Mexico, 29-31 August 2012; pp. 570-575. 
8. Casillas, M.V.; Garza-Castañón, L.; Puig, V. Model-based leak detection and location in water distribution networks considering an extended-horizon analysis of pressure sensitivities. J. Hydroinf. 2013, (in press).

9. Yassine, A.; Ploix, S.; Flaus, J.M. A method for sensor placement taking into account diagnosability criteria. Int. J. Appl. Math. Comput. Sci. 2008, 18, 497-512.

10. Rosich, A.; Sarrate, R.; Nejjari, F. Optimal Sensor Placement for FDI Using Binary Integer Linear Programming. In Proceedings of the 20th International Workshop on Principles of Diagnosis, DXâĂŹ09, Stockholm, Sweden, 14-17 June 2009; pp. 235-242.

11. Krause, A.; Leskovec, J.; Guestrin, C.; Vanbriesen, J.; Faloutsos, C. Efficient sensor placement optimization for securing large water distribution networks. J. Water Resourc. Plan. Manag. 2008, 134, 516-526.

12. Aral, M.; Guan, J.; Maslia, M. Optimal design of sensor placement in water distribution networks. J. Water Resour. Plan. Manag. 2010, 136, 5-18.

13. Sarrate, R.; Nejjari, F.; Rosich, A. Sensor Placement for Fault Diagnosis Performance Maximization in Distribution Networks. In Proceedings of the 18th Mediterranean Conference on Control and Automation (MED), Barcelona, Spain, 3-6 July 2012; pp. 1-6.

14. Christodoulou, S.; Gagatsis, A.; Xanthos, A.; Kranioti, S.; Agathokleous, A.; Fragiadakis, M. Entropy-based sensor placement optimization for waterloss detection in water distribution networks. Water Resourc. Manag. 2013, 27, 4443-4468.

15. Rokach, L.; Maimon, O. Clustering Methods. In Data Mining and Knowledge Discovery Handbook; Springer: New York, USA, 2005; pp. 321-352.

16. Fujiwara, O.; Khang, D.B. A two-phase decomposition method for optimal design of looped water distribution networks. Water Resourc. Res. 1990, 26, 539-549.

17. Zheng, F.; Zecchin, A.C.; Simpson, A. Self-adaptive differential evolution algorithm applied to water distribution system optimization. J. Comput. Civil Eng. 2013, 27, 148-158.

18. Bolognesi, A.; Bragalli, C.; Marchi, A.; Artina, S. Genetic heritage evolution by stochastic transmission in the optimal design of water distribution networks. Adv. Eng. Softw. 2010, 41, 792-801.

19. Rossman, L. EPANET 2 User's Manual; United States Envionmental Protection Agency: Washington, DC, USA, 2000.

20. Koza, J.R. Survey of Genetic Algorithms and Genetic Programming. In Proceedings of the Wescon 95-Conference Record: Microelectronics, Communications Technology, Producing Quality Products, Mobile and Portable Power, Emerging Technologies, San Francisco, CA, USA 7-9 November 1995; pp. 589-594.

21. Goldberg, D. Genetic Algorithms in Search, Optimization, and Machine Learning; Addison-Wesley: Reading, MA, USA, 1989. 


\title{
GPR-Based Water Leak Models in Water Distribution Systems
}

\author{
David Ayala-Cabrera, Manuel Herrera, Joaquín Izquierdo, Silvia J. Ocaña-Levario and \\ Rafael Pérez-García
}

\begin{abstract}
This paper addresses the problem of leakage in water distribution systems through the use of ground penetrating radar (GPR) as a nondestructive method. Laboratory tests are performed to extract features of water leakage from the obtained GPR images. Moreover, a test in a real-world urban system under real conditions is performed. Feature extraction is performed by interpreting GPR images with the support of a pre-processing methodology based on an appropriate combination of statistical methods and multi-agent systems. The results of these tests are presented, interpreted, analyzed and discussed in this paper.
\end{abstract}

Reprinted from Sensors. Cite as: Ayala-Cabrera, D.; Herrera, M.; Izquierdo, J.; Ocaña-Levario, S.J.; Pérez-García, R. GPR-Based Water Leak Models in Water Distribution Systems. Sensors 2013, 13, $15912-15936$.

\section{Introduction}

Water is valuable, but challenging to manage. It has been calculated that many water distribution systems (WDSs) around the world lose more than 40 percent of the clean water pumped into the distribution system because of leaks before that water reaches end consumers [1]. By reducing the amount of water leaked, WDS managers can reduce the amount of money and energy wasted on producing and pumping water, increase system reliability and more easily satisfy present and future consumer needs. Having access to sufficient information regarding leaks is a complex task. Many water utilities struggle to measure and locate leaks in their distribution networks.

Improved leakage management in WDSs is one of the intelligent solutions that can make a difference. The use of different types of smart sensors to gather data and the application of advanced analytics could provide valuable information on the location of leaks in the network. Specifically, non-destructive methods, such as ground penetrating radar (GPR), can help locate primordial leaks and, so, help resolve the problem, while avoiding social and economic costs.

In [2], a review of the various pipeline inspection techniques most commonly used in WDSs systems and wastewater collection is performed. These techniques are divided into four groups: (a) visual techniques; (b) electromagnetic and radio frequency techniques; (c) acoustic and vibration techniques; and (d) other techniques. Closed-circuit television (CCTV) and sewer scanner and evaluation technology (SSET) are highlighted in the first group (visual techniques). The second group (electromagnetic and radio frequency techniques) consists of magnetic flux leakage (MFL), eddy current technique, hydroscope technology (HT), rapid magnetic permeability scan (RMPS), low frequency electromagnetic field (LFEM), passive magnetic fields (PMFs), time domain ultra wideband (UWB) and ground penetrating radar (GPR). The third group (acoustic and vibration techniques) includes sonar, vibro-acoustics, impact echo/spectral analysis of surface waves and correlator and listening sticks for leaks. The last group (other techniques) includes infrared thermography, continuous wave Doppler sensing technique, laser surveys, combined 
techniques (broadband electromagnetics/wave impedance probe (WI), pipe inspection real-time assessment technique (PIRAT) and the Sahara Project.

Among these techniques, the most popular for locating leaks in water supply systems are those included in the acoustic and vibration technique group, infrared thermography and GPR $[3,4]$. Acoustic methods detect the acoustic wave generated by the leak based on correlation analyses of the wave velocity of the sound emitted by the pipe being inspected. Such methods are widely used to identify leaks in fluid-filled metal pipes [5]. The main drawback of the aforementioned methods is their inefficiency in detecting leaks in non-metallic pipes (e.g., Polyvinyl chloride (PVC) pipes) [6]. Infrared methods detect thermal contrasts caused by the difference of temperature between ground and water. However, even though easy to implement, these methods produce errors when there are considerable differences in temperature. Furthermore, it is not possible to use these techniques in summer and winter, due to the absence of significant differences between ground and water [4]. GPR is shown as an effective nondestructive tool that favors inspection of WDS by demarcating on GPR image (radargrams) contrasts between the leaked water and the surrounding soil that are caused by differences in dielectric characteristics [7,8].

The use of GPR as a method for locating leaks in WDS has become more widespread in recent years. In this sense, there is fieldwork, such as [9], performed on urban pipe sections. Pre-processing of the obtained images is performed by using low-pass filters to identify leaking PVC pipes. Another representative fieldwork is reported in [10]. In this case, a plastic pipe (PVC) was drilled and buried in the ground; and, an analysis was made using raw images. Likewise, there is fieldwork using a combination of methods. Such is the case of [11], which combines GPR assays with electrical potential and geochemical assays to detect leaks in non-pressurized non-metallic pipes. In this case, leaks are identified from raw GPR images. Laboratory tests are also employed in finding leaks using GPR. Works, such as [12,13], concentrate on plastic pipes. In these cases, pre-processing includes background removal and image filtering, respectively. A combination of survey work conducted both in the field and in the laboratory is presented in [14]. In this paper, various tests on leaks in plastic and metallic pipes were performed. In this work, Kirchhoff migration and the Hilbert transform were used as pre-processing methods. These assays are promising with respect to the use of GPR in finding leaks in WDS. However, most of these assays are based only on the location and interpretation of hyperbolas generated either in raw or pre-processed images.

Identifying leaks by GPR images is not an easy task and requires a high level of expertise by the operator. Added complications include the complex spatial arrangement of many networks, along with the steady growth in the supply infrastructure of cities. These aspects greatly increase the difficulty in using and interpreting data obtained with GPR in detecting leaks and analyzing the results, thus reducing the potential for solving problems and increasing the need for highly qualified personnel. This paper attempts to address this issue with the extraction of features visually and numerically. To this purpose, laboratory tests are performed in which we seek to extract features of water leakage from GPR images. Feature extraction is performed by interpreting the GPR images with the support of a pre-processing methodology based on an appropriate combination of statistical methods and multi-agent systems. Subsequently, these features are observed in a field test on a real water leak-case in a WDS. The ultimate goal of these processes is to extract features to feed intelligent 
automatic processes for the (automatic) detection of leaks in WDS using GPR images. This research thus seeks to encourage the use of these tools in finding leaks by non-highly qualified personnel and, thus, promoting improved management of WDS.

The paper is organized as follows. In the first section, we have presented a brief introduction and cited relevant papers. The second section presents the characteristics of the tests performed. The third section presents an analysis of typical GPR images by locating and interpreting hyperbolas. The fourth section discusses the numerical contrast images of a non-leaking laboratory empty pipe and then a series of tests with water and leakage. The use of a race-agent algorithm as a pre-processing tool for GPR images and its application to the images of the laboratory tests are proposed in Section 5. The following section presents a contrast analysis, similar to that performed in Section 4, but using pre-processed images. 3D models of the interpretations obtained in Sections 4 and 5 are then presented in Section 7. The eighth section presents the identification of a leak in fieldwork. Finally, a section of conclusions closes the document.

\section{Data Capturing: Design and Layout of the Laboratory Tests}

This section presents the layout of the laboratory tests. In this set of tests, a pipe commonly used in WDSs is buried in dry soil in a tank (see Figure 1a). The characteristics of the buried pipe are: (a) PVC; (b) diameter of $100 \mathrm{~mm}$; (c) length of $0.95 \mathrm{~m}$; (d) hole drilled for simulating the leak in the central part of the pipe; (e) two points for water input (herein termed WI) and output (herein termed WO) with connections at the ends. A wooden tank measuring $1.0 \mathrm{~m} \times 1.0 \mathrm{~m} \times 0.70 \mathrm{~m}$ was used. After the pipe was positioned, its supports were removed, and it was then covered with dry soil. The surface of the tank was covered with a polypropylene plate. Eleven paths parallel to the $x$-axis and another eleven paths parallel to the $y$-axis were marked on this plate. These 22 paths were spaced $0.10 \mathrm{~m}$ apart (see Figure 1b) and, so, formed the sampling grid (see Figure 1c). Onto each line of this mesh, the GPR antenna was slipped. The image produced by sliding the antenna is termed a profile in this document (see, for example, s5 in Figure 1c). Additionally, we distinguish between horizontal and vertical profiles when referring to the profiles parallel to the $x$ - and the $y$-axes, respectively.

The GPR equipment used in each survey corresponds to a commercial monostatic antenna with a central frequency of $1.5 \mathrm{GHz}$. The parameters of the equipment correspond to $120 \mathrm{traces} / \mathrm{s}$, 512 samples/trace and $20 \mathrm{~ns} / 512$ samples. Two tests with the aforementioned features of 22 profiles were performed. These two tests are differentiated one from the other in that the first was without water inside the pipe (nor leaked water), while the second test had water inside the pipe and leaked water. These two situations are called initial and final state, respectively, in this paper. 
Figure 1. Laboratory layout. WI, water input; WO, water output.

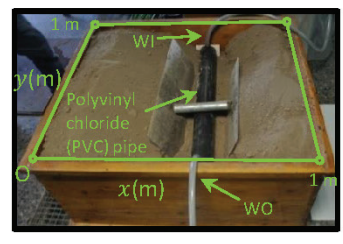

(a)

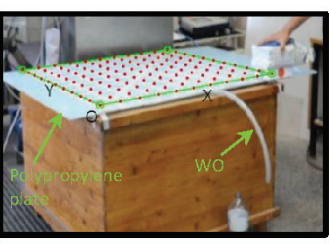

(b)

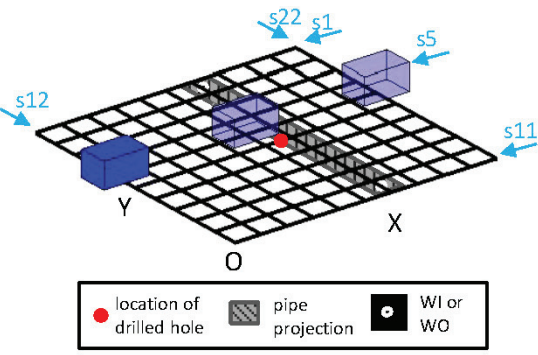

(c)

\section{Analysis of the Location and Identification of Hyperbolas from the Raw Images}

This section presents a typical analysis to identify anomalies (leaks in this case) in the raw images. This analysis consists in locating and identifying hyperbolae in the GPR images, according to the scheme presented in the previous section. These tests are performed on the horizontal and vertical profiles for both the initial and end states. To facilitate the interpretation of raw images in Sections 3.1 and 3.2, various examples of target forms are sketched in Figure 2. Incidentally, Figure $2 \mathrm{a}$ corresponds to Figure 5a, Figure $2 \mathrm{~b}$ to Figure $5 \mathrm{c}$ and Figure $2 \mathrm{c}$ to Figure $5 \mathrm{~g}$.

Figure 2. Identifiable forms in raw images in Sections 3.1 and 3.2: (a) distorting vertical strip; (b) hyperbola; (c) triangles.

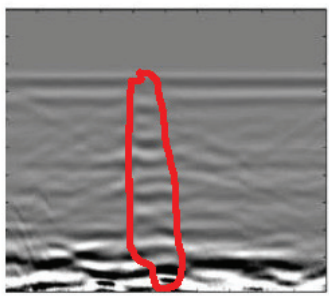

(a)

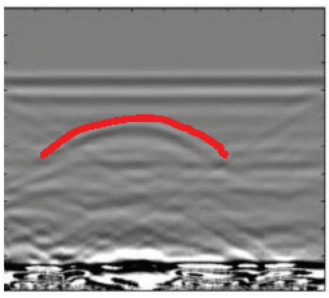

(b)

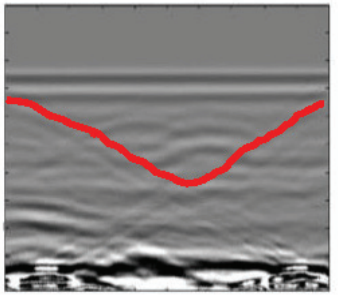

(c)

\subsection{Initial State: Raw Images}

The resulting images from the horizontal and vertical profiles of the tests performed in the laboratory for the initial state are presented in Figures 3 and 4, respectively. 
Figure 3. Horizontal profiles: raw images for the initial state. (a-k) are profiles s1 to s11, respectively.

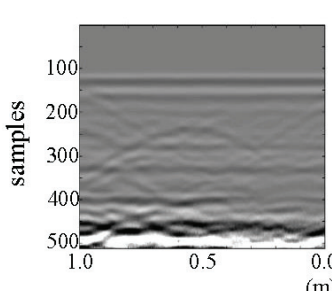

(a)

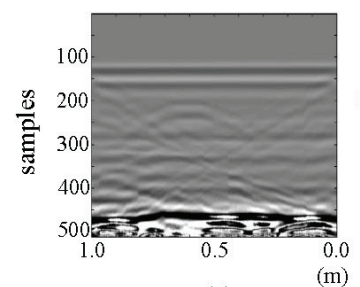

(e)

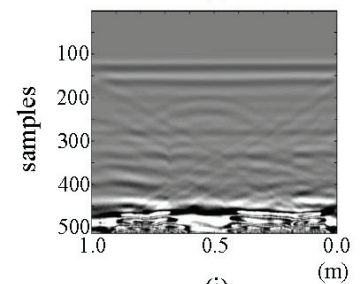

(i)

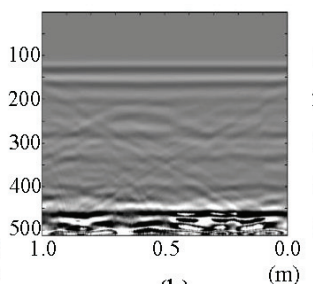

(b)

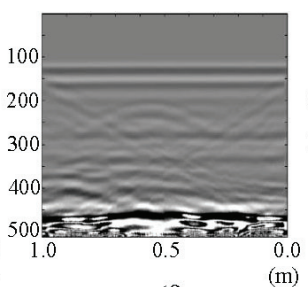

(f)

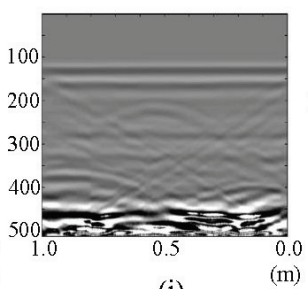

(j)

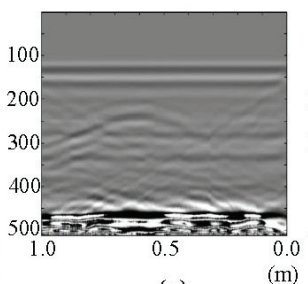

(c)

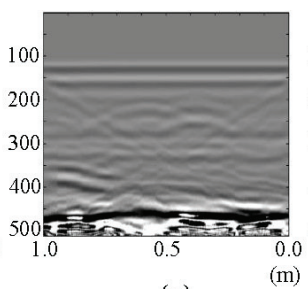

(g)

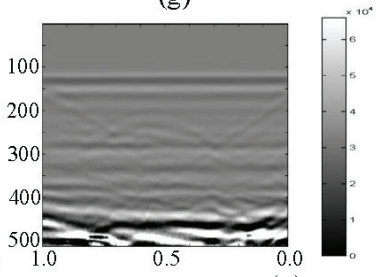

(k)

(m)

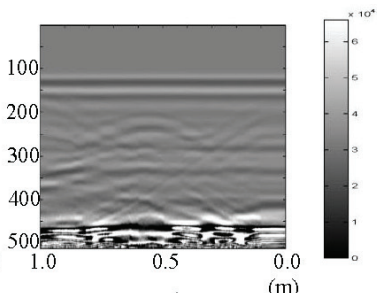

(d)

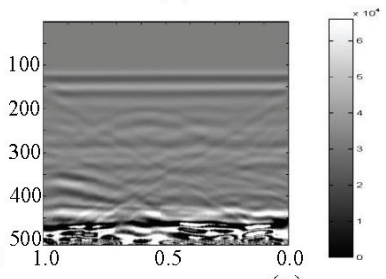

(h)

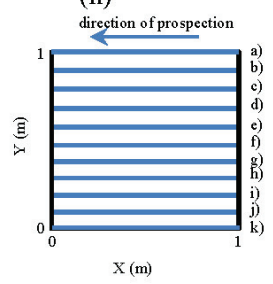

$\mathrm{X}(\mathrm{m})$

All the images presented in Figure 3 exhibit a hyperbola, whose center is located at approximately $0.6 \mathrm{~m}$ in all cases. This location coincides with the location of the PVC buried pipe. In this same figure, in parts (f), (g) and (h), a new anomaly, a new hyperbola located to the right of the hyperbola associated with the buried pipe, can be identified. The new hyperbola increases in intensity in Figure $3 \mathrm{~g}$. In this case, it is known that this anomaly cannot represent the leak, since we are still analyzing the initial state. However, in an uncontrolled case, this feature could lead to confusion.

All the images depicted in Figure 4 show how the hyperbola representing the pipe in Figure 3 no longer appears. This is consistent with the schematic configuration proposed for the test, given that vertical profiles are being considered. However, formations (triangles) inclined from the tank walls, which were also seen in the horizontal profiles (Figure 3), can be observed. In all the images in Figure 4, a gradually spanning structure can be observed: for parts from (a) to (d) between samples 275-325; for part (e) between samples 250-325; for (f) to (h) between samples 225-275; for part (i) between samples 275-325; and for (j) between samples 300-325. This pattern corresponds to the signal response for the now longitudinally placed pipe. 
Figure 4. Vertical profiles: raw images for the initial state. (a-k) are profiles s12 to s22, respectively.

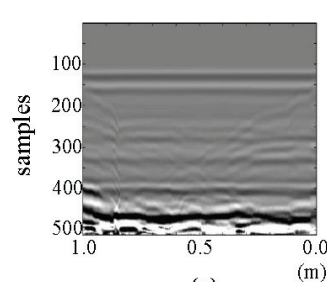

(a)

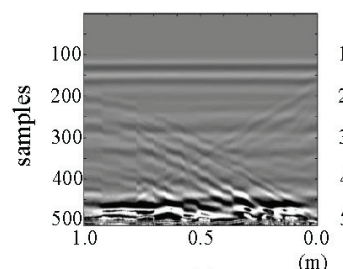

(e)

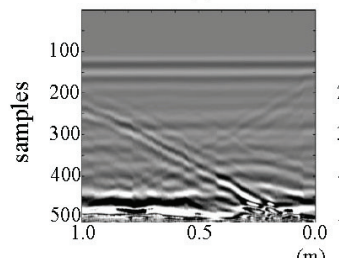

(i)

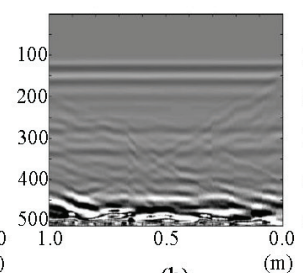

(b)

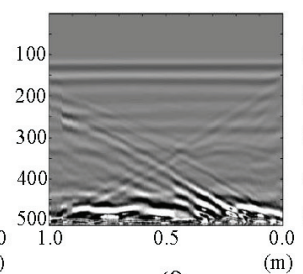

(f)

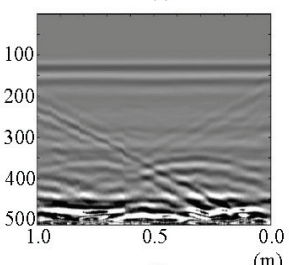

(j)

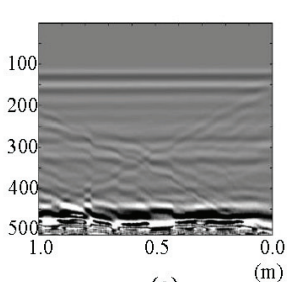

(c)

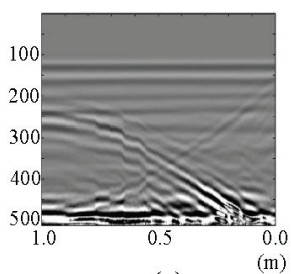

(g)

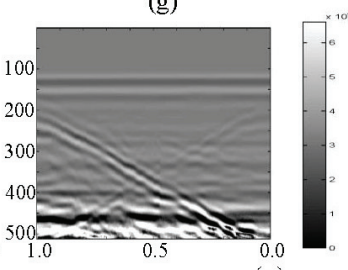

(k)

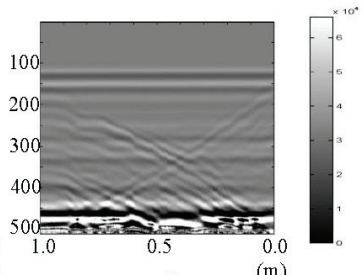

(d)

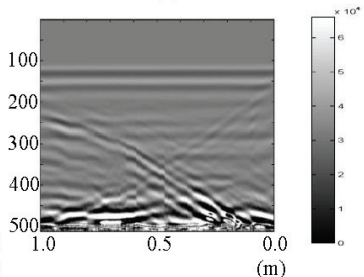

(h)

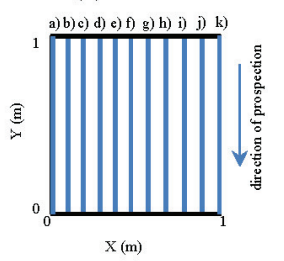

\subsection{Final State: Raw Images}

The resulting images of horizontal and vertical profiles of the tests performed in the laboratory for the final stage are presented in Figures 5 and 6, respectively.

In Figure 5, in images (a) and (k), a vertical strip that distorts both images can be seen between 0.5 and $0.6 \mathrm{~m}$. This effect is attributed to the presence of water in WI and WO. This effect, which is more accentuated in (a), is more clearly seen when contrasting these images with their corresponding images in Figure 3: the area containing the deformed strip in Figure 5 contained (in Figure 3) the shape of the hyperbola previously identifying the buried pipe. In images (b) and (j) of Figure 5, a new hyperbola surrounded by another upper hyperbola (pipe initial hyperbola) can be identified. By observing profiles from (b) to (j), we can say that the hyperbola we had considered to represent the pipe decreases in intensity as the position of the profile nears the point of the leak. In effect, the hyperbola is almost invisible in images (e) and (f). We can also observe how in image (f), two hyperbolas are identified (very faintly), where, initially, only one hyperbola appeared. In (g), this second hyperbola as obtained in the initial state (close to the hyperbola of the pipe) is observed. If we had not previously had the image without water, this second hyperbola would surely have been interpreted as a leak. This shows the need to extract patterns for interpretation and leak detection in water supply systems and, so, avoid common mistakes in field interpretation. This additional hyperbola may result from any object (such as stones) near the pipeline. 
Figure 5. Horizontal profiles: raw images for final state: (a-k) are profiles s1 to s11, respectively.

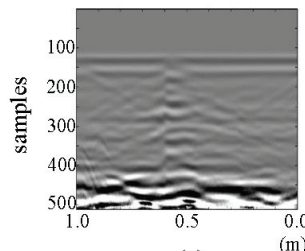

(a)

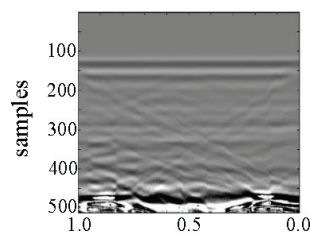

(e)

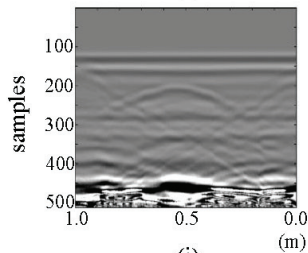

(i)

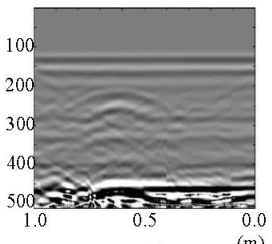

(b)

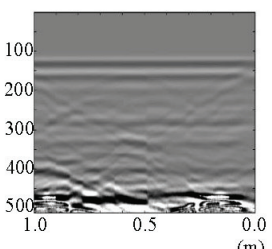

(f)

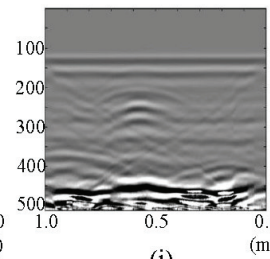

(i)

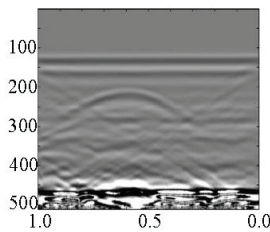

(c)

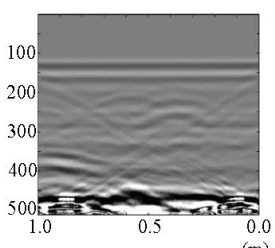

(g)

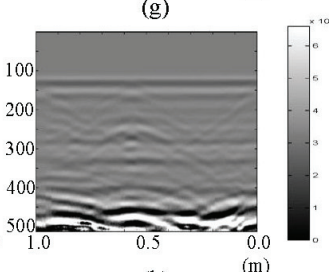

(k)

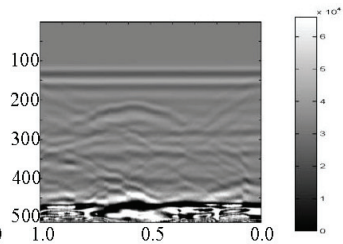

(d)
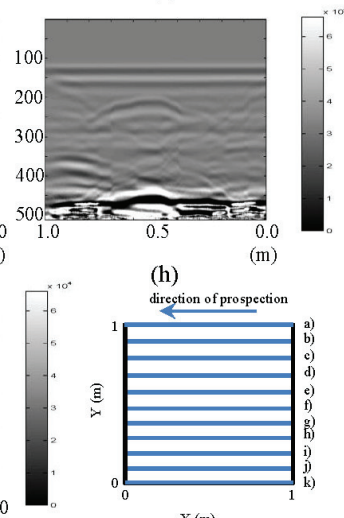

$\mathrm{X}$ (m)

Figure 6. Vertical profiles: raw images for final state: (a-k) are profiles s12 to s22, respectively.

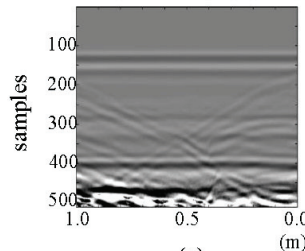

(a)

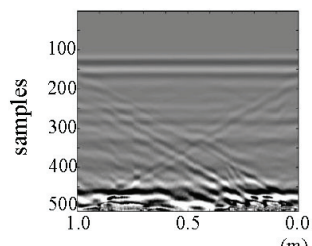

(e)

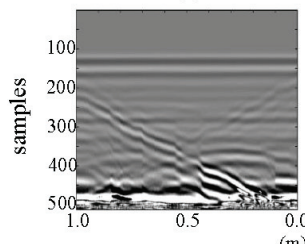

(i)

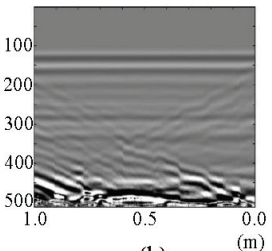

(b)

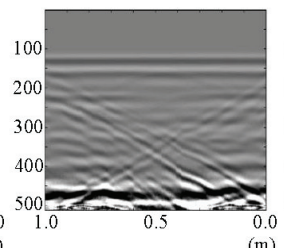

(f)

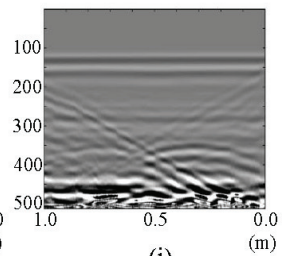

(i)

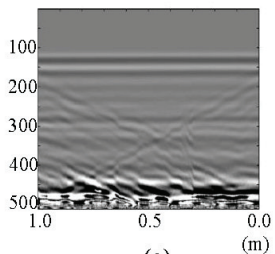

(c)

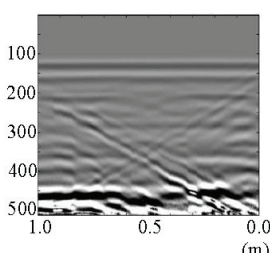

(g)

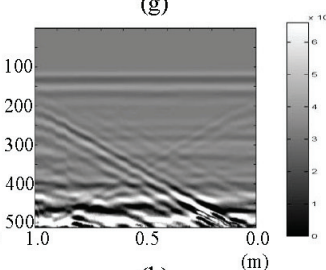

(k)

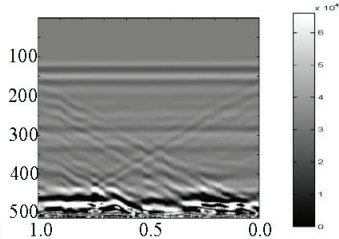

(d)

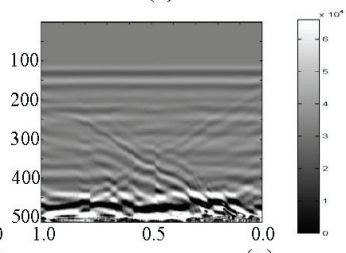

(h)

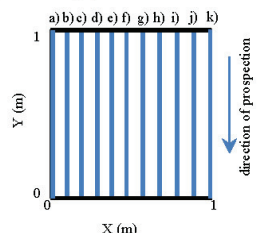


Images in Figure 6, in contrast, show similar characteristics to the respective images of the initial state. However, in (f) and (g), a contraction of the area we had identified as the pipe in Figure 4 can be observed between 0.5 and $0.4 \mathrm{~m}$ (distance axis) and samples 225 and 275 (depth axis). This contraction is better observed in image g), which corresponds to the vertical section closer to the pipe. Furthermore, the position of the contraction is consistent with the location of the leakage point in the test.

After these various analyses of the different horizontal and vertical profiles corresponding to the leakage, we observe that identifying leaks directly from raw images is difficult. In fact, even considering the a priori information about the location of the leak, it is a challenge to distinguish all the above-mentioned features.

\section{Analysis: Contrast between Raw Images for the Initial and Final States}

In this section, since a suitable metric is available, we present a comparison between the initial and the final state of the tests performed for each raw image. Figures 7 and 8 show the differences in horizontal and vertical raw images, respectively.

Figure 7. Horizontal profiles: contrasting raw images for initial and final states. (a-k) are profiles s1 to s11, respectively.

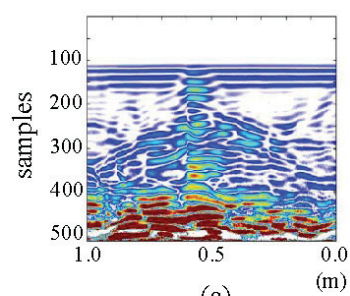

(a)

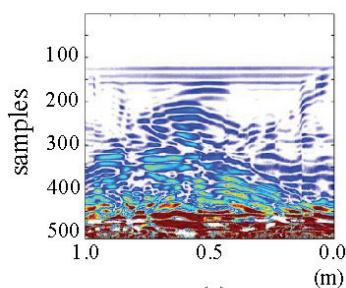

(e)

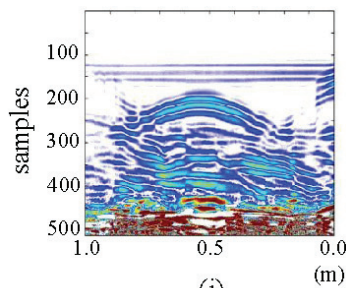

(i)

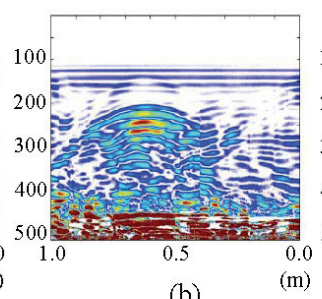

(b)

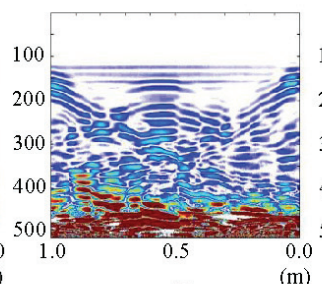

(f)

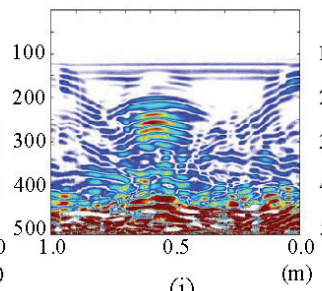

(j)

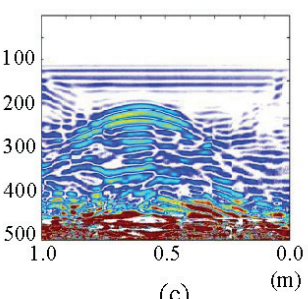

(c)

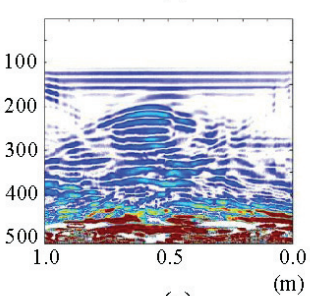

(g)

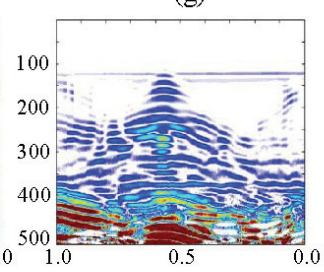

(k)

(m)

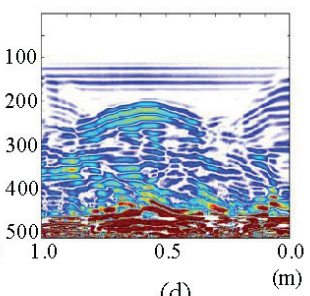

(d)

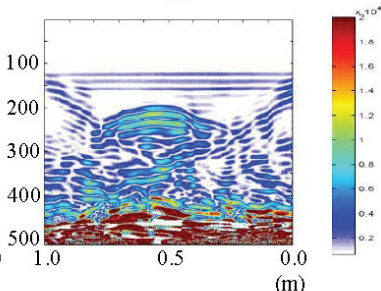

(h)

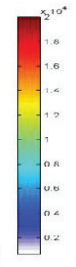

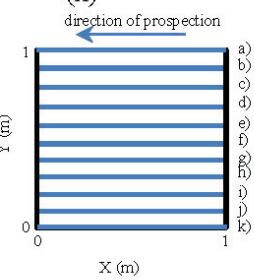

$\mathrm{X}(\mathrm{m})$

The images in Figure 7 show the presence of water, which is the differentiating factor between the images of the initial and the final state. In parts (a) and (k) of the figure, the water in WI and 
WO can be easily seen. The water contained in the pipe can be observed in pictures from (b) to (j). The brightest part now corresponds to the water that has replaced the air contained in the pipe in the initial state. This shows that the difference in intensity identifies the specific fluid (air or water, in our case) contained in the pipe. Similarly, it is observed that the color intensity decreases as the location of the images nears profile (f) (the profile closest to the leak). Moreover, in images (e) to $(\mathrm{g})$, between 0.4 and $0.6 \mathrm{~m}$ and between samples 150 and 200, a hyperbola that was not readily detectable in the respective raw images (and was missed in the interpretations) is now easily observed. This hyperbola is defined, according to the observation made in situ, by the rise in water leaked by capillarity. For images (e) and (f), the water contained in the pipe, on the right side (of the image), is demarcated with correspondingly lower intensity. This would indicate that the leak is running from left to right. This is because in that area, there is a mixture of soil and water, and this causes lower color intensity. A decrease in color intensity in the demarcated area of the pipe when approaching the leakage point (profile f) can also be observed. Additionally, some deformation is also observed below all the areas demarcated by the contrast, which is the result of the expected convolution (bearing in mind that the measurements are performed at depth).

Figure 8. Vertical profiles: contrasting raw images for initial and final states. (a-k) are profiles s12 to s22, respectively.

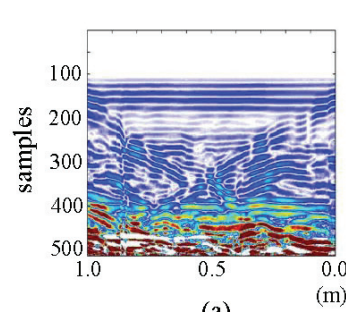

(a)

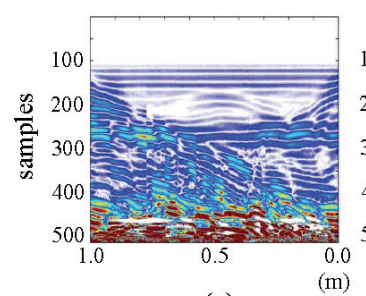

(e)

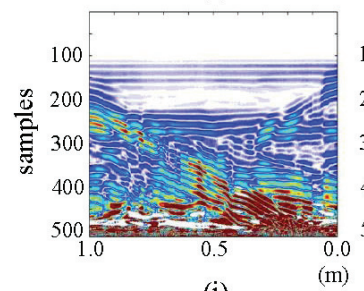

(i)

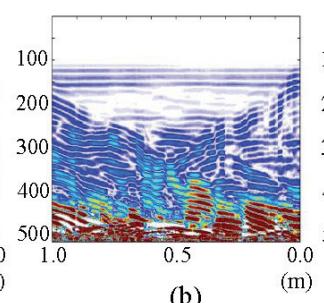

(b)

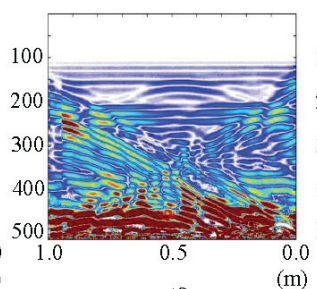

(f)

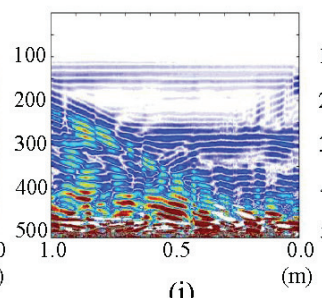

(j)

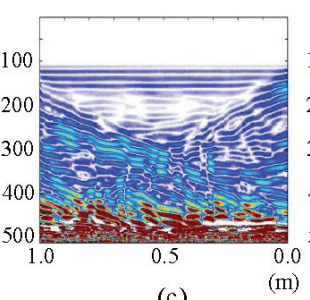

(c)

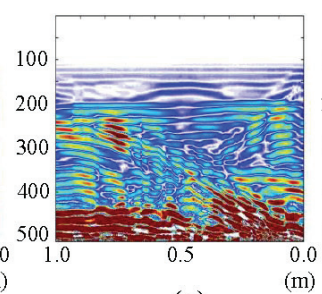

(g)

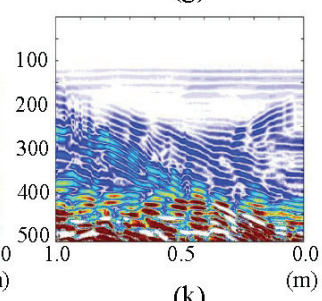

(k)

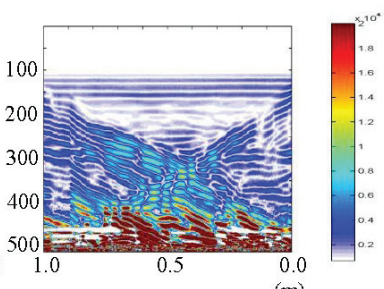

(d)

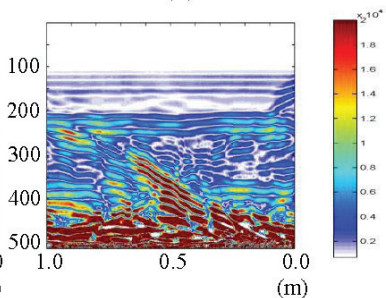

(h)

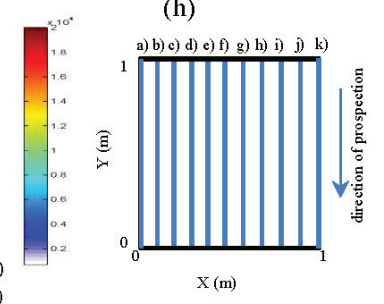

In Figure 8, image ( $\mathrm{g}$ ) (which is the closest to the pipe and to the leakage point), an increase in the color intensity in the strip running almost through the entire profile can be observed. This strip, between samples 200 and 250, shows the addition of water in the pipe. In this same image and for 
the same strip, the effect described regarding the same image for the final state can also be seen. This is the effect of the contraction of the pipe at the point where the leak is located. In addition, there is a hyperbola at the top (a feature not seen in its respective final state image). Similarly, in (h) (the profile closest to the pipe and on the path where the pipe is located), the strip has lower color intensity than in $(\mathrm{g})$, while being higher than in the closer profiles. Observe that this strip fades and diminishes in height, in the direction from (i) to (j) and from (f) to (e).

Likewise, a hyperbola in (e), (f) and (g), which gains color intensity as the point of leakage is approached (image g)) can be seen. From (f) to (e), the hyperbola loses strength and increases in size as a result of the increasing distance from the point of the leak.

\section{Analysis of Location and Identification of Anomalies in Pre-Processed Images}

In this section, we present the principles of the pre-processing algorithm used in this work. Subsequently, this algorithm is applied to the images obtained in the laboratory for the initial and final state. The results will be compared and analyzed later.

\subsection{Multi-Agent System for the Pre-Processing Algorithm}

The analysis carried out in Section 3 is based on intensity differences demarcated by the wave amplitude generated in the images after the passage of the signal through various subsurface strata. The analysis we present in this section is based on time characteristics. In this analysis, the peaks (both maxima and minima) of the waves generated are extracted. The trend of the path of each trace and the average value of peak-to-peak time are studied. The basic principle assumes that the field is homogeneous, and thus, there should be a clear correspondence between the various peaks obtained. However, it should be noted that although the material is homogeneous, in practice, the measured values are different, even though very close together. Consequently, very different values demarcate anomalies in the image. These peaks were first extracted and numbered according to their occurrence in the trace. They were then placed in their respective positions in distance, and finally, the last value is used to fill an array of a certain size (512 in this work) (see Figure 9).

To quickly obtain this matrix, the algorithm proposed by [15] was used. In this algorithm, we obtain the above-mentioned values by means of a system based on multiple entities (agents) that search peaks by simulating a race. A multi-agent system consists of a population of autonomous entities (agents) situated in a shared structured framework (environment). The system is based on such tools as game theory (e.g., setting the agent preferences by means of a utility function), economics, biology, as well as artificial intelligence algorithms [16]. Agents operate independently, but are also able to interact with the environment and coordinate with other agents. This coordination may imply cooperation if the agent society works towards common objectives. Thus, in a cooperative community, agents usually have individual capabilities, which, when combined, will lead to solving the entire problem. However, cooperation is not always possible, and there are instances where agents are competitive and have divergent goals. In this later case, the agent should also take into account the actions of others [17]. However, even if agents are able to act and achieve their goals by 
themselves, it may be beneficial to partially cooperate and form coalitions for a better performance. When coordinating activities, either in a cooperative or a competitive environment, negotiation may prove a suitable method for solving conflicts among agents. Negotiation may be seen as the process of identifying interactions based on communication and reasoning with regard to the state and intentions of other agents [18].

Figure 9. Principles of the ground penetrating radar (GPR) image pre-processing.

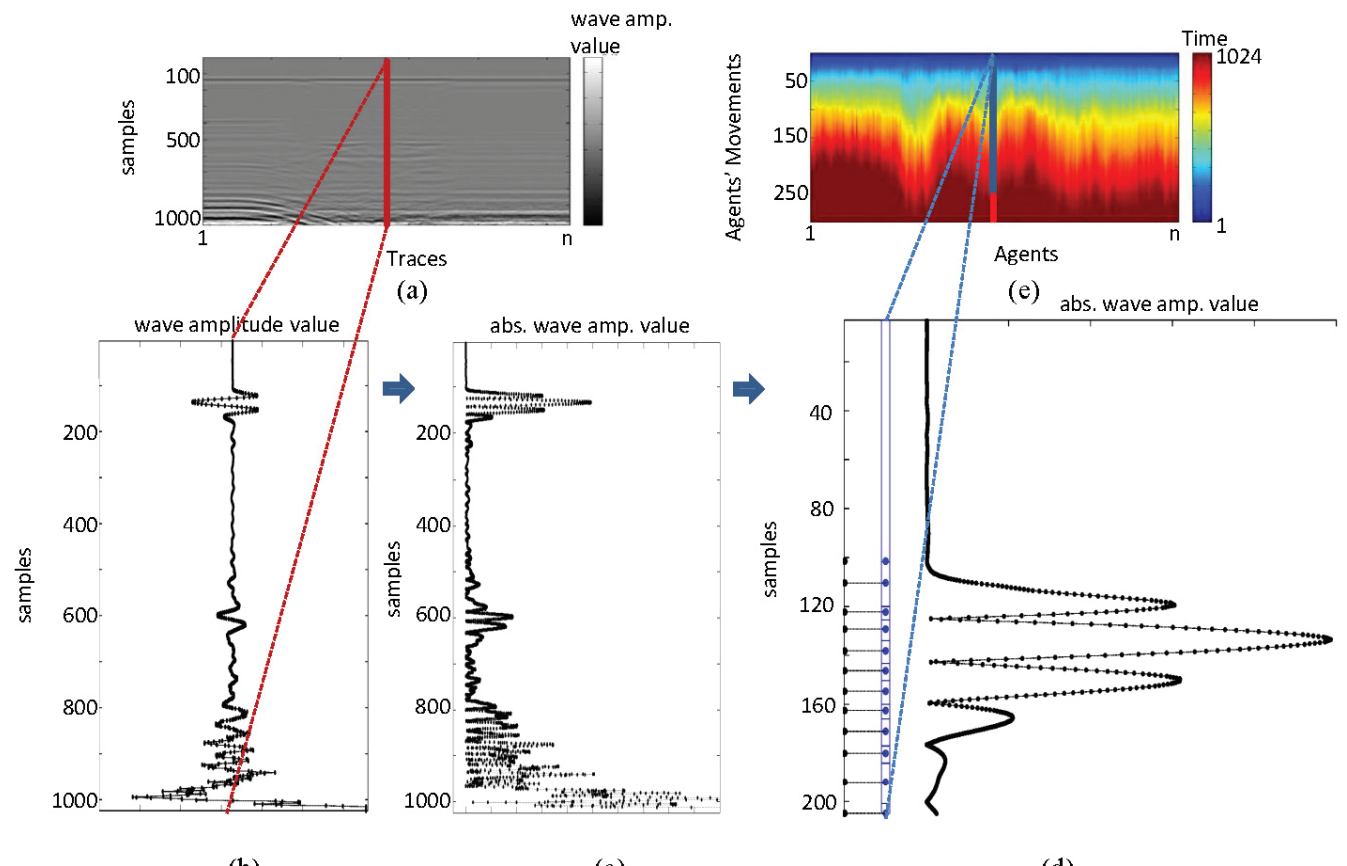

(b)

(c)

(d)

The pre-processing of GPR images used in this document was proposed in [15] and was termed an agent race. The algorithm has been developed in MatLab, is based on game theory and uses the multi-agent paradigm [16]. Agent racing provides an interpretation and a grouping method for data from GPR radargrams. In this pre-process, we reduce the amount of data in the initial radargram, while preserving its initial properties and the most relevant data, so that its ability to identify buried objects through suitable visualizations is preserved. The multi-agent approach makes analysis much quicker. The input to this algorithm is the resulting radargram of the GPR prospection, which consists of an $m \times n$-sized matrix. The $n$-traces, of length $m$, that are generated are used in this work as parallel tracks for the $n$-agents to run. The race is an endurance test for the agents with a prize consisting in advancing one position depending on the effort made. Efforts are based on wave amplitude values in each column of the matrix (radargram). The agent race is comprised of two phases: (a) warm-up; and (b) competition. The race lasts a total time of $t=t_{w}+t_{r}=m$, where $t_{w}$ is the warm-up time and $t_{r}$ the competition time. The movements of an agent in $t_{r}$ are conditioned by the changing trend of the wave amplitude of the trace the agent runs. The race ends when time $t$ has elapsed. The winner is the agent who manages to obtain more movements (reward) during this time. The output (output 
1) of this process is a matrix of the size $m 1 \times n$, where $m 1=$ the maximum number of movements. The columns of this matrix describe the movement of the agents in relation to the competition. In this paper, we restrict the output (output 2) to the matrix of the size $t_{r} \times n$ that collects the movements performed by each agent during the competition (see Figure 10). In this work, the various movements developed by the agents are termed time lines.

Figure 10. Scheme for the agent-race algorithm.

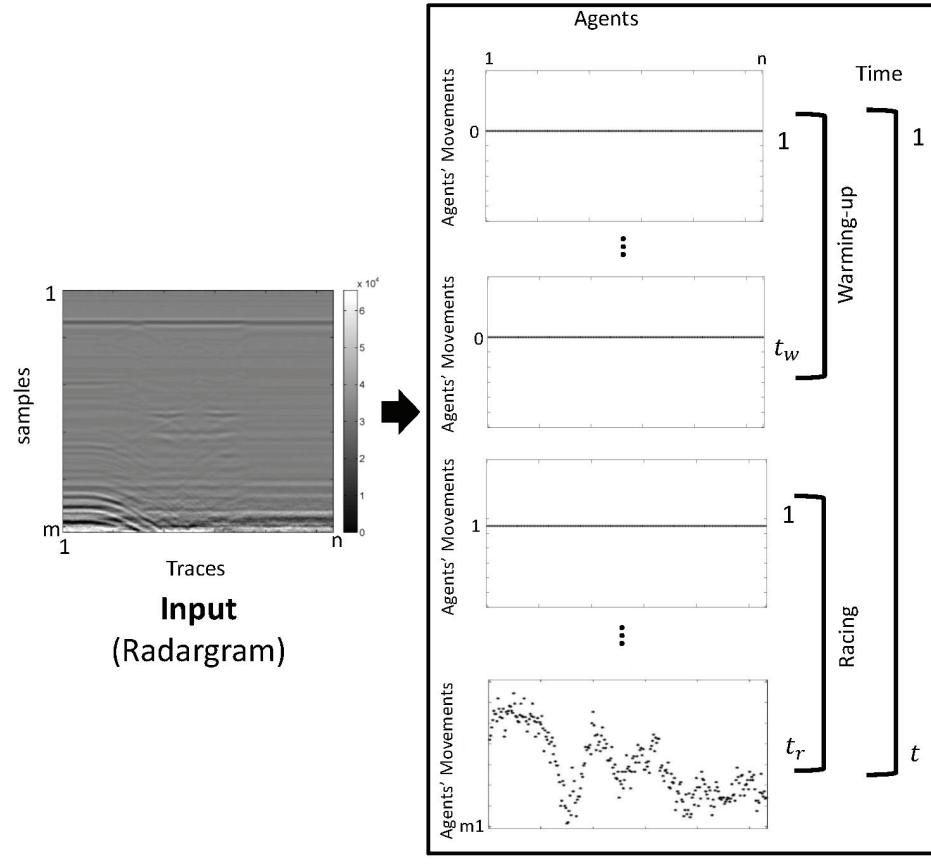

Agent race

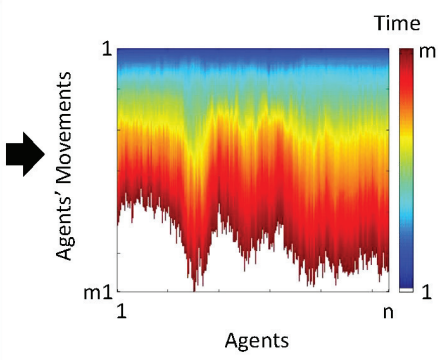

Output1

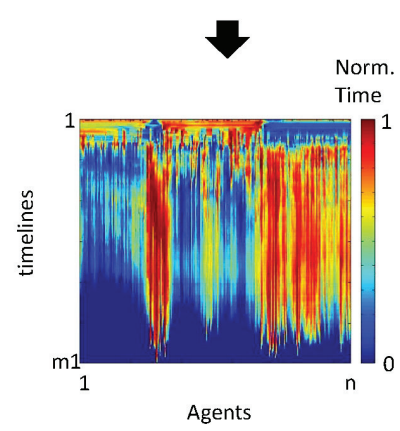

Output2

On each time line, times obtained in the competition for each agent are ordered decreasingly. These time lines are then normalized. This produces output 2 (Figure 11), which is the matrix we use herein. This eliminates the delays caused by the gap between rows and enables more intense highlighting of anomalies that are very small and difficult to observe in raw images. However, care must be taken with this regularization, because although the anomalies are highlighted, their intensity is determined in relation to the ground prospected in each profile. This can cause visual errors in interpretation. Yet, even if this occurs, the interpretation based on the identification of various forms will enable zones of interest to be delimited, thus facilitating more complex analyses.

Again, to facilitate the interpretation of preprocessed images in Sections 5.2 and 5.3, examples of the more relevant forms are sketched in Figure 2. In this case, Figure 12a corresponds to Figure 15a, Figure $12 \mathrm{~b}$ to Figure $15 \mathrm{c}$, Figure $12 \mathrm{c}$ to Figure $15 \mathrm{~g}$ and Figure $12 \mathrm{~d}$ to Figure $16 \mathrm{f}$. 
Figure 11. Output 2 building: (a) output 1; (b) time line; (c) ordered time line; (d) ordered and normalized time line; and (e) output 2.

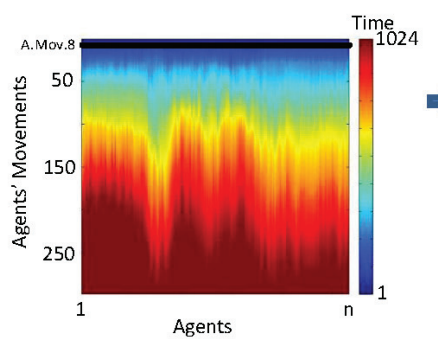

(a)

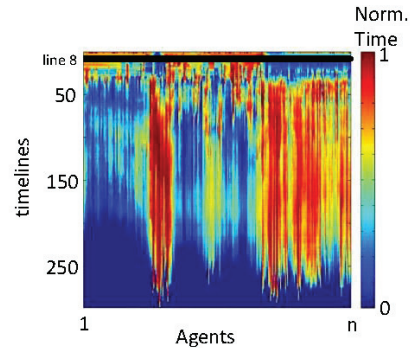

(e)

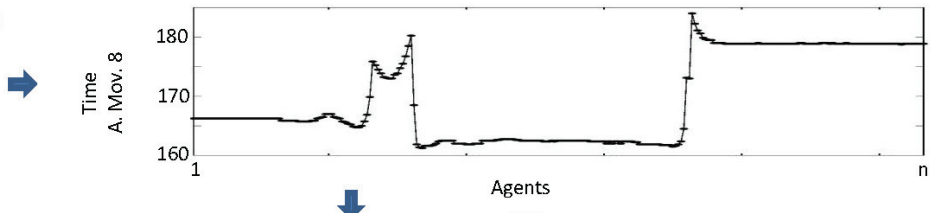

(b)

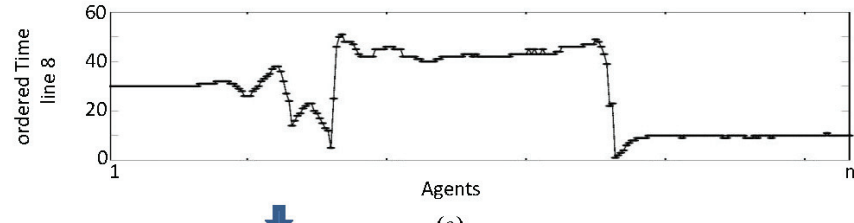

(c)

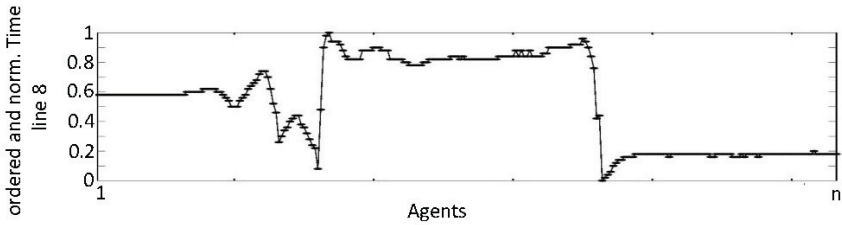

(d)

Figure 12. Identifiable target forms in pre-processed images in Section 5.2 and 5.3: (a) conical shape for water input (WI) and output (WO); (b) ellipse; (c) tank and measuring plate contours; and (d) water leak shape.

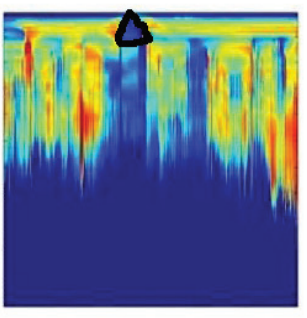

(a)

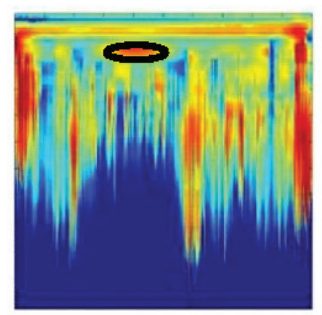

(b)

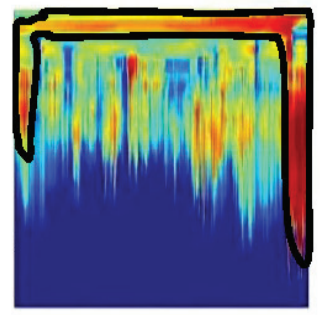

(c)

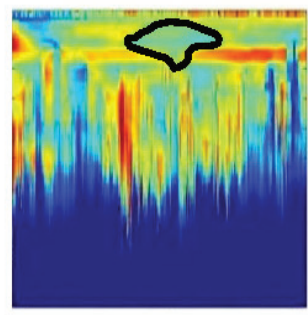

(d)

\subsection{Initial State: Pre-Processed Images}

The pre-processed images derived from the tests performed in the laboratory for the initial state show the horizontal and vertical profiles presented in Figures 13 and 14, respectively. 
Figure 13. Horizontal profiles: pre-processed images for the initial state. (a-k) are profiles s1 to s11, respectively.

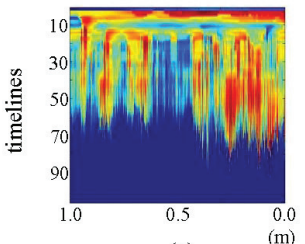

(a)

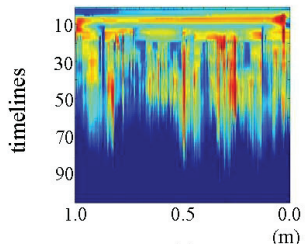

(e)

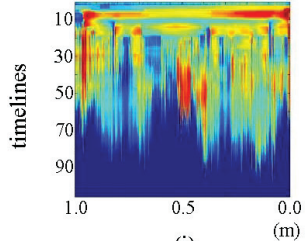

(i)

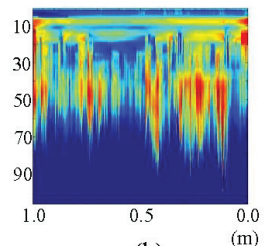

(b)

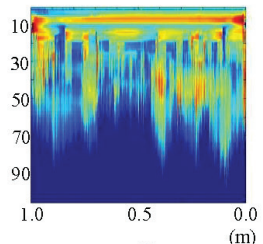

(f)

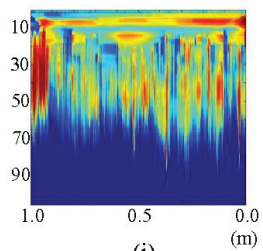

(i)

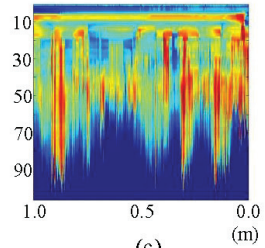

(c)

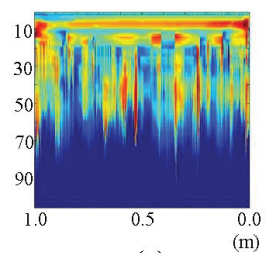

(g)

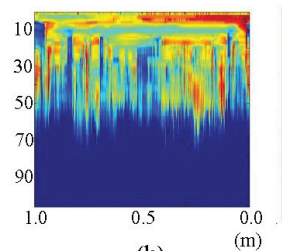

(k)

(III)

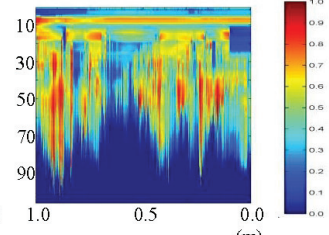

(d)

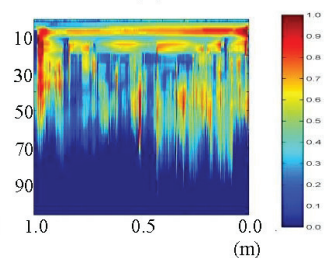

(h)

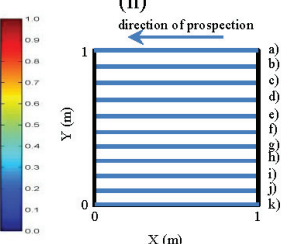

Figure 14. Vertical profiles: pre-processed images for the initial state. (a-k) are profiles s12 to s22, respectively.

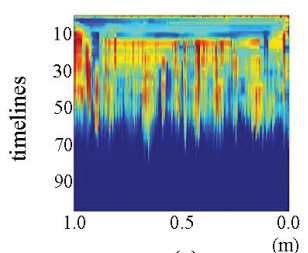

(a)

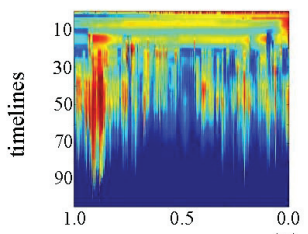

(e)

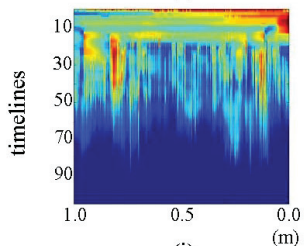

(i)

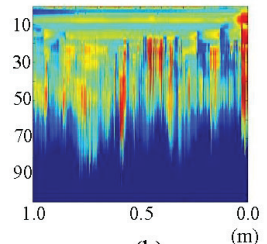

(b)

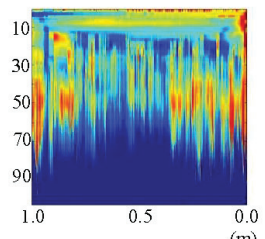

(f)

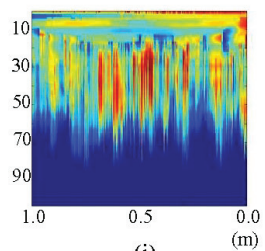

(i)

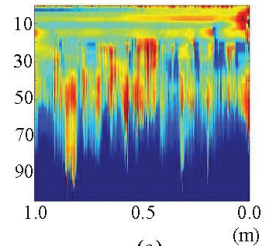

(c)

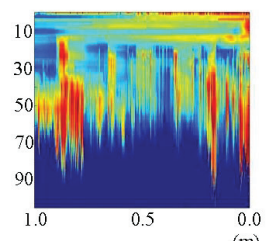

(g)

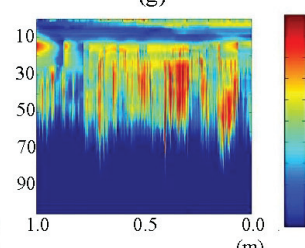

(k)

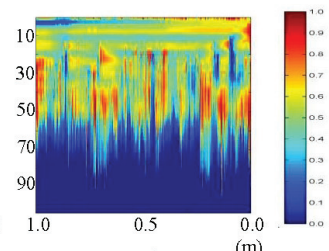

(d)

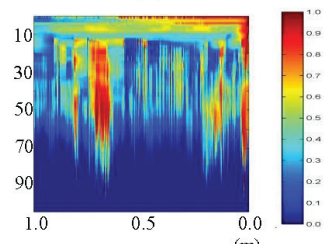

(h)

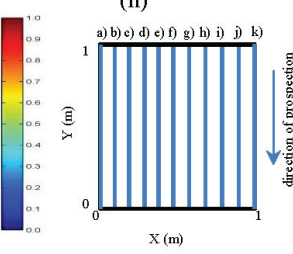


In Figure 13, an ellipse-shaped object is observed exactly in the place where the pipe was placed. In each image, it can be observed how the color intensity decreases from the center of the ellipse to its border. In images (a) and (k), an additional conical shape may be observed above this ellipse, which is not noticeable in the corresponding raw images. This image corresponds to WI and WO. Additionally, at about time line 10 in all the images (with variable intensity), the development of a horizontal figure can be observed that crosses all the images, which, at each of its two ends, joins an intense zone that demarcates a vertical figure. This horizontal formation is the polypropylene plate used for sliding the antenna during measurement, and the vertical forms correspond to the walls of the tank.

In the images in Figure 14, a certain relationship between the different color intensities of each profile can be observed. The same characteristic areas of the tank and the measuring plate, already observed in the pre-processed horizontal profiles, are demarcated with greater intensity. However, the inclination of this area, which was previously horizontal, can be observed from images (f) to (h). Moreover, in the central part, we note the non-appearance of the ellipse that appeared in the pictures above.

\subsection{Final State: Pre-Processed Images}

The pre-processed images derived from the tests performed in the laboratory for the final state, showing the horizontal and vertical profiles, are presented in Figures 15 and 16, respectively.

Figure 15. Horizontal profiles: pre-processed images for the final state. (a-k) are profiles s1 to s11, respectively.
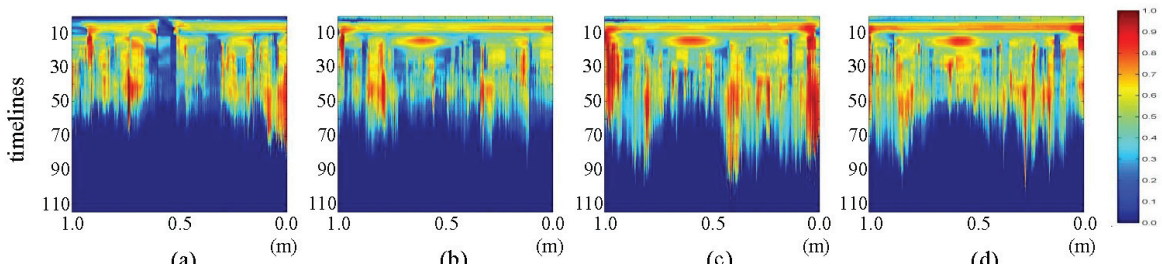

(a)

(b)

(c)
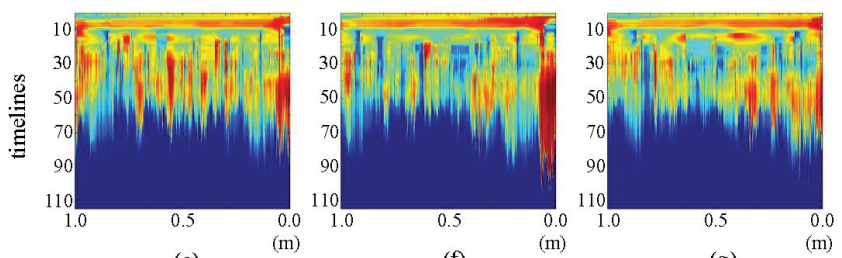

(d)

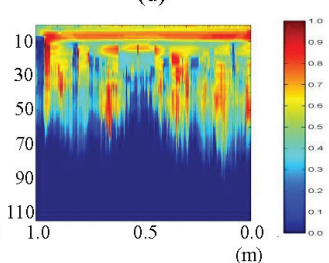

(g)

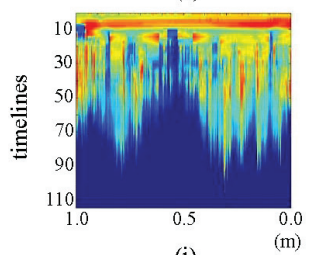

(f)

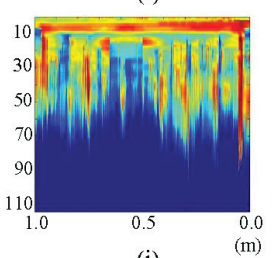

(i)

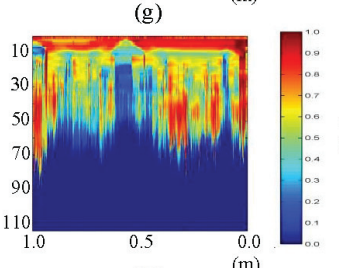

(h)

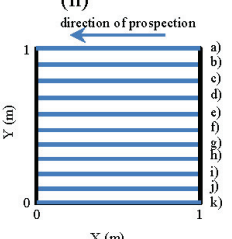

(k)

$\mathrm{X}(\mathrm{m})$

(i)

(i)

(II) 
Figure 16. Vertical profiles: pre-processed images for the final state. (a-k) are profiles s12 to s22, respectively.

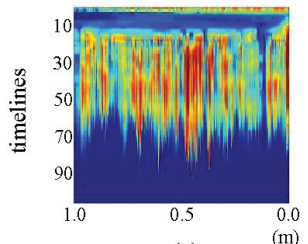

(a)

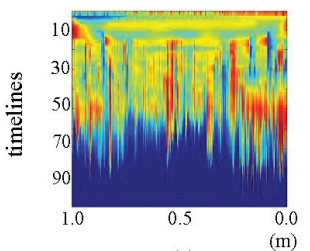

(e)

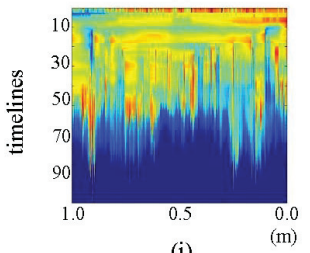

(i)

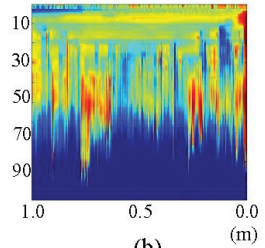

(b)

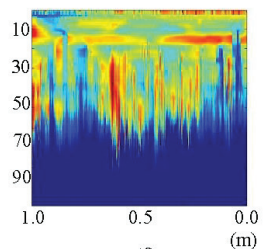

(f)

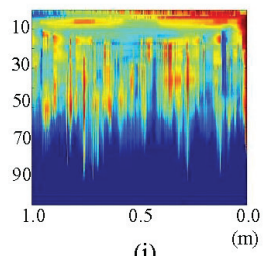

(i)

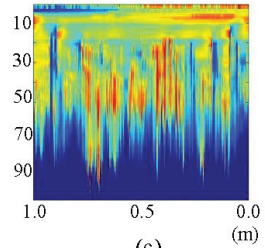

(c)

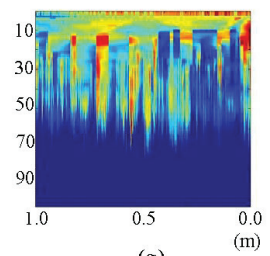

(g)

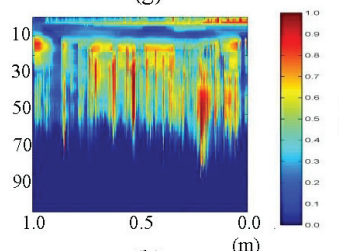

$(\mathrm{k})$

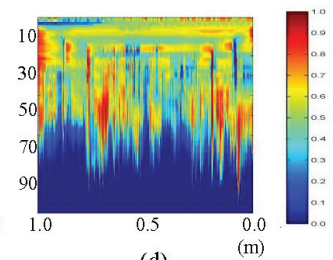

(d)

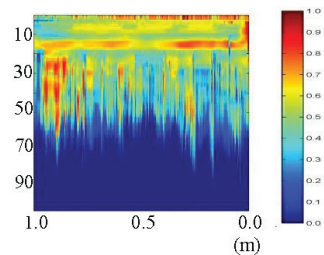

(h)

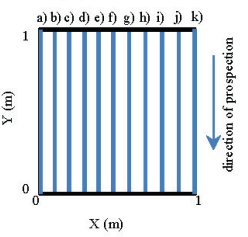

In Figure 15, it can be seen how the ellipse observed in the corresponding initial state image gains color intensity with the addition of water in the pipe for all the images. Likewise, in images (e) and (f), such intensity decreases. This feature was observed in the profile of the raw images and can be seen more clearly in the pre-processed images. Likewise, it can be observed in (a) and (k) that the intensity of the structure representing WI and WO increases with the addition of water to the system. It should be noted that this feature is clearly observed only after contrasting the initial and final states and not directly in the raw images. However, in the pre-processed image, this feature is easily identifiable. In images $(\mathrm{g})$ to $(\mathrm{k})$, the central ellipse loses intensity toward $0 \mathrm{~m}$ and fades in its central part. The contours generated by the tank and measuring plate are better delineated in these images.

In Figure 16, a discontinuity of color intensity in the form of an ellipse between images (e) to (g) can be observed. This discontinuity grows as it approaches profile (g) (location of the leak). It should be mentioned that this was not at all observable in the corresponding raw image. Now, in the pre-processed images, it becomes apparent.

\section{Analysis: Contrast between Pre-Processed Images}

In this section, we present a number of contrasts between the pre-processed profiles for the initial and final states. In Figure 17 and 18, contrasts for the horizontal and vertical profiles, respectively, are presented. 
Figure 17. Horizontal profiles: contrast between pre-processed images. (a-k) are profiles s1 to s11, respectively.

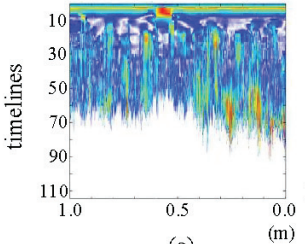

(a)

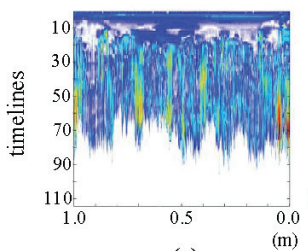

(e)

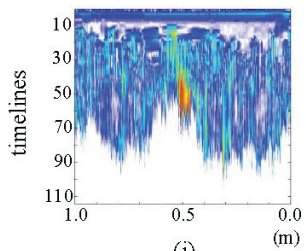

(i)

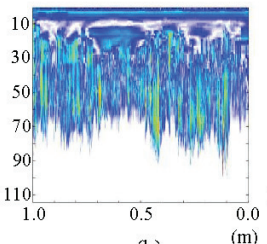

(b)

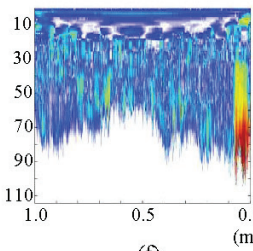

(f)

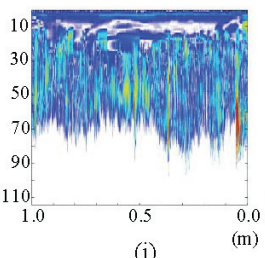

(j)

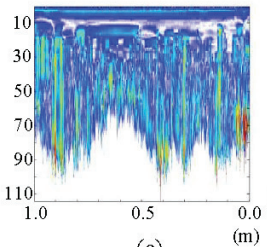

(c)

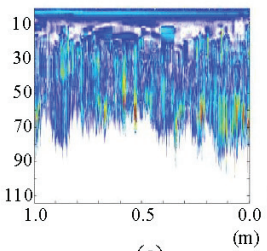

(g)

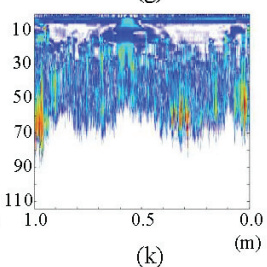

(k)

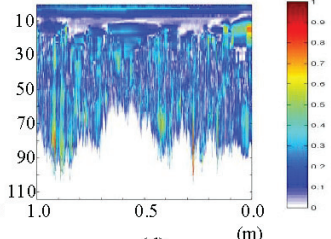

(d) (m)

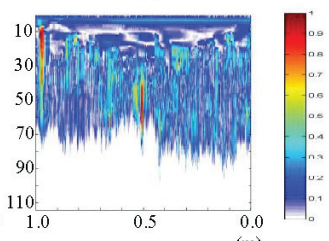

(h)

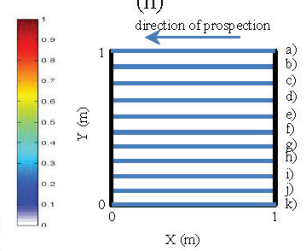

Figure 18. Vertical profiles: contrast between pre-processed images. (a-k) are profiles s12 to s22, respectively.

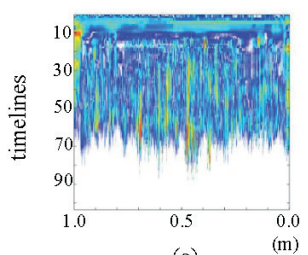

(a)

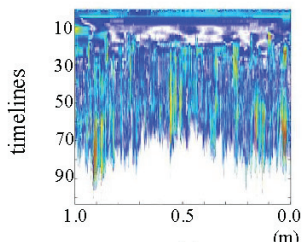

(e)

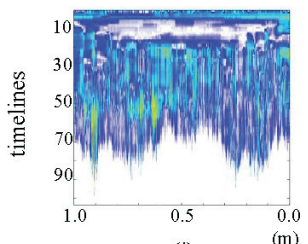

(i)

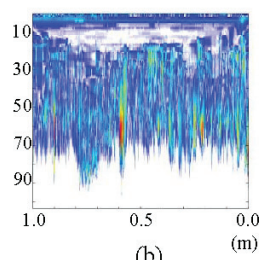

(b)

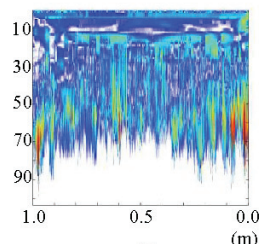

(f)

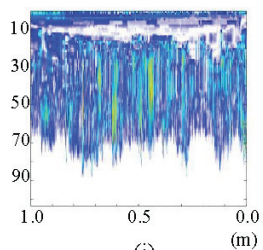

(j)

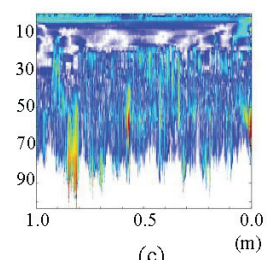

(c)

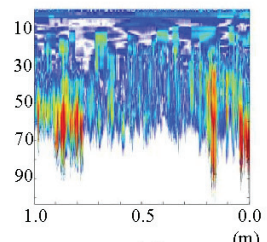

(g)

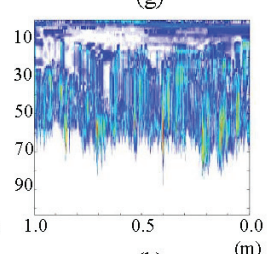

(k)

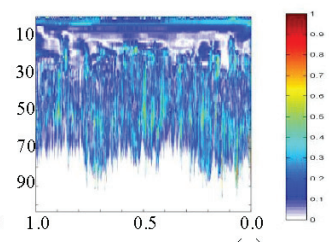

(d)

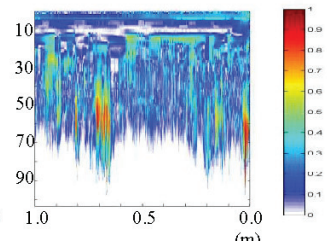

(h)

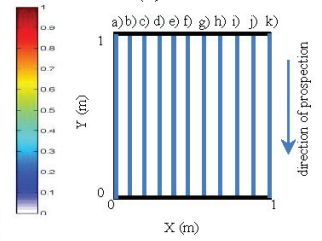


In Figure 17, images (a) and (k), the mark that was observed in the interpretations already made can be easily seen. This mark corresponds to the introduction of water at WI and WO. Additionally, in the same pictures, an anomaly in the form of an ellipse at the bottom can be appreciated. This shape corresponds to the water in the pipe. Moreover, various profiles reveal the formation of the ellipse whose center is highly intense and which fades toward the ends. In these profiles, we see how the color intensity decreases as we near the central profile (leak location profile). Similarly, in images (e) and (g), one can observe the formation of a new ellipse that is located just above the ellipse corresponding to the water in the pipe. We also note that in image (f), this form is a hyperbola enveloping the ellipse corresponding to the area of water in the pipe. This form corresponds to leaked water, part of which has risen by capillarity to the surface of the ground.

In Figure 18, images (e), (f) and (g), one can observe the generation of an ellipsoidal shape that increases in size and consistency, as it travels from (e) to $(\mathrm{g})$. Note that profile $(\mathrm{g})$ is closest to the location of the leak.

\section{3D Comparison of the Analysis of Contrasts between Raw and Pre-Processed Images}

In this section, we perform the extraction of the contours of the images resulting from the previously conducted contrasts. With these contours, two 3D models of contrasting performances in raw images and pre-processed images of the water in the pipe and leaked water are obtained. Contour extraction and the corresponding 3D representation have been developed in MatLab. In Figure 19, we present a simple scheme of one of the processes, whose two main steps are described as follows:

Figure 19. Example of 3D model construction: (a) GPR interpretation of raw data; (b) contour extraction; and (c) 3D model rendering.

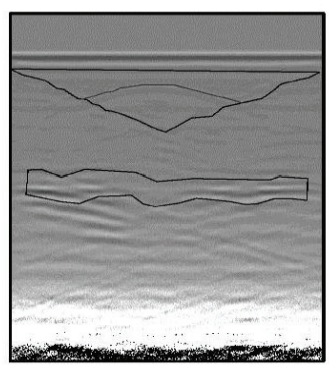

(a)

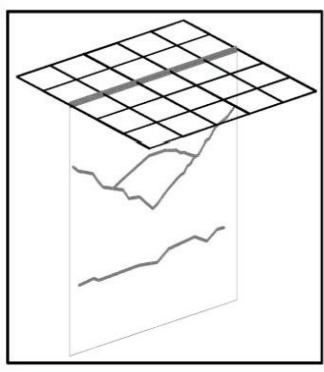

(b)

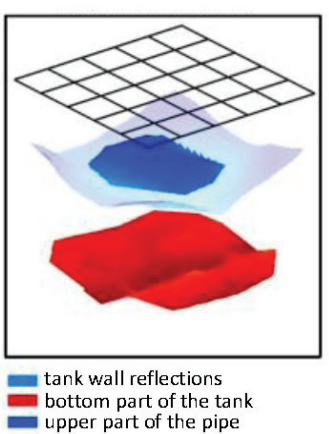

(c)

$G P R$ interpretations. Contours that can represent features of interest in the prospected underground are extracted either from the raw or the pre-processed images. When interpreting images of radargrams, the features most commonly analyzed are hyperbolas. They are demarcated by the color intensity within the image. A non-automatic process is proposed in this document with the aim of determining the feasibility of the interpretation of GPR data. In addition, we are interested 
in observing if these interpretations are reliable and can gain relevance for understanding features of leaks in WDSs . However, if this process is effective, it could be implemented automatically.

Fusion process and 3D model. The fusion of the GPR image interpretations is achieved by their projection onto a common space. Firstly, a classification of common zones in the GPR interpretations that may correspond to specific sections of the area or volume is performed. Then, a mesh in the 3D space is constructed. Each of these volumes or surfaces is then added to a common space, shrinking, thus, into the 3D model. There are a number of applications to achieve this kind of fusion employed in photography or painting [19], which can be adapted to generate 3D models of the GPR interpretations.

In Figure 20, we present the 3D models.

Figure 20. Comparison between $3 \mathrm{D}$ models generated from the interpretation of the horizontal and the vertical profiles contrasting the initial and the final states: (a) 3D model obtained from raw images; (b) 3D model obtained from pre-processed images.

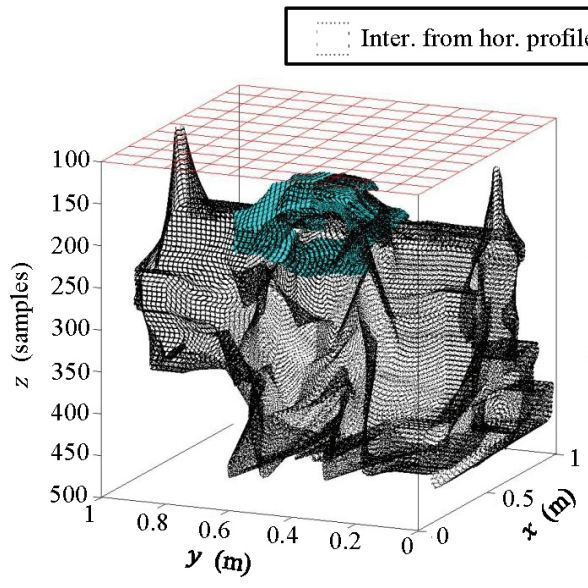

(a)

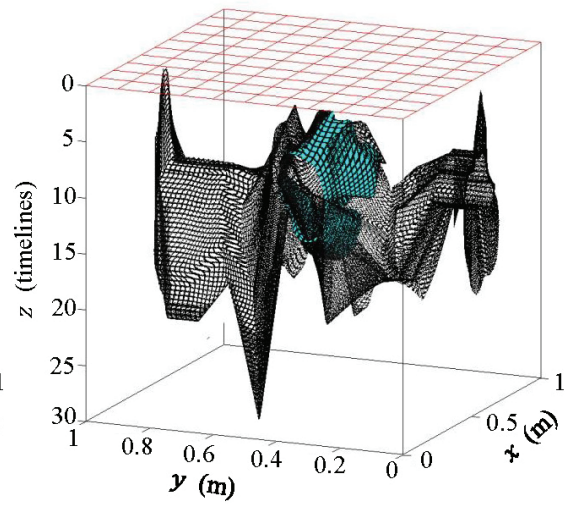

(b)

The 3D models presented in Figure 20 show clear agreement with the schematic approach performed. It is also clear that the models are related, although their depth axis differs. Obviously, this is because they come from different images. In both cases, the water in WI and WO and the water in the pipe can be clearly observed. The leakage is also identified, but more consistently in (b) than in (a). It can be seen that 3D models produce more measurable results (numerically) and more comprehensible interpretation than flat images.

\section{Analysis of Field Images: A Case Study}

This section discusses the use of the multi-agent methodology, proposed in the previous section, in a real case. The fieldwork was conducted in an urban water supply system on a section of pipe, whose location was known. Using a geophone, turbulence points (where it was supposed that there was a water leak) were detected, and tests on the area near the pipe were performed using GPR. 
The roadway is hydraulic concrete and the pipe is $400-\mathrm{mm}$ cast iron. A structure of paths similar to those in the laboratory tests was demarcated on the roadway (Figure 21a). The grid-mesh was $0.50 \mathrm{~m}$. The area was $4.0 \mathrm{~m}$ by $2.0 \mathrm{~m}$ long in $x$ and $y$, respectively. The profiles obtained with the survey were named sf 1 to sf 8 and sf 9 to sf13 for horizontal and vertical lines, respectively (Figure 21b). It should be mentioned that the pipe is located in the sf11 profile. The GPR equipment used and the capture parameters correspond to the same features of the equipment used in the laboratory tests. However, in this case, the antenna used had a central frequency of $400 \mathrm{MHz}$.

Figure 21. Layout for the fieldwork.

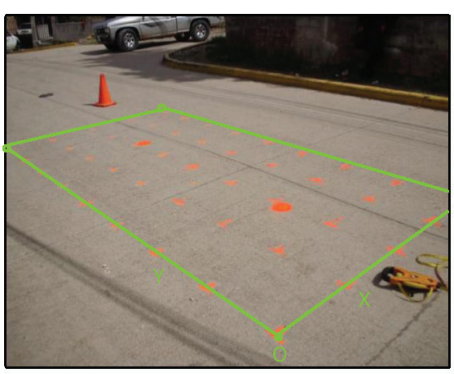

(a)

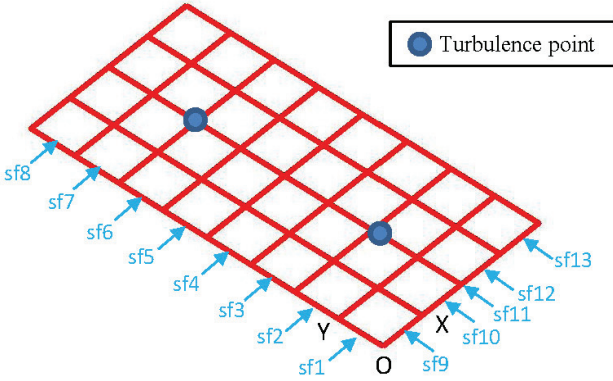

(b)

In Figures 22 and 23, the raw images for the horizontal and vertical profiles, respectively, are presented.

Figure 22. Horizontal profiles: raw images. (a-h) are profiles sf1 to sf8, respectively.

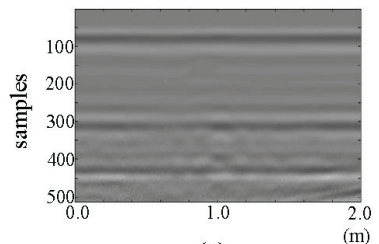

(a)

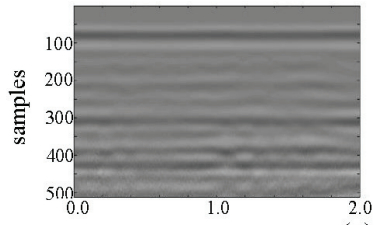

(d)

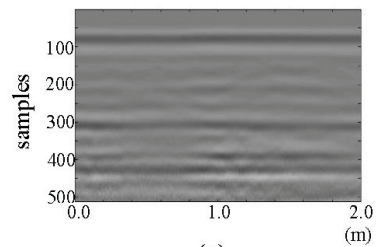

(g)

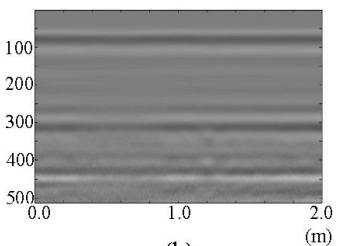

(b)

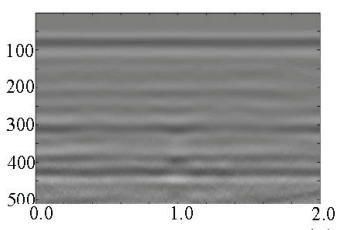

(e)

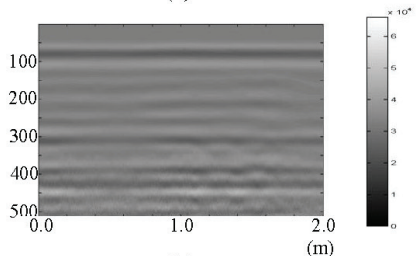

(h)

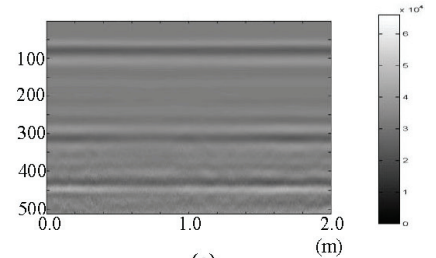

(c)

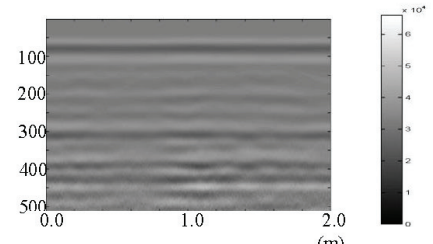

(f)

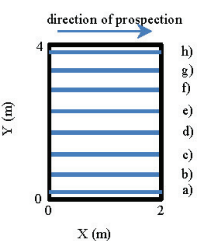

$\mathrm{X}(\mathrm{m})$ 
Figure 23. Vertical profiles: raw images. (a-e) are profiles sf9 to sf13, respectively.

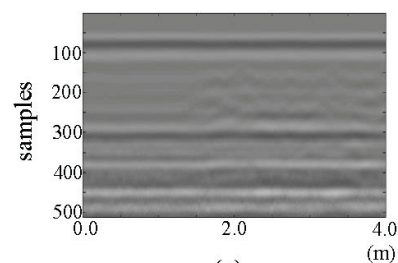

(a)

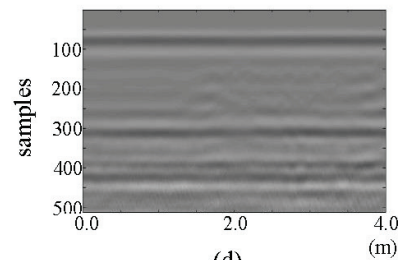

(d)

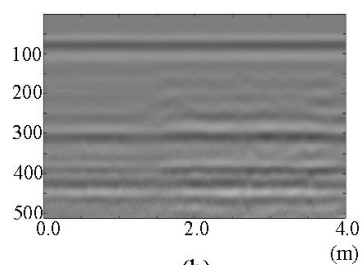

(b)

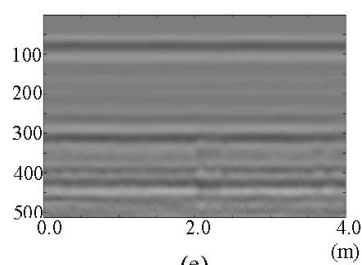

(e)

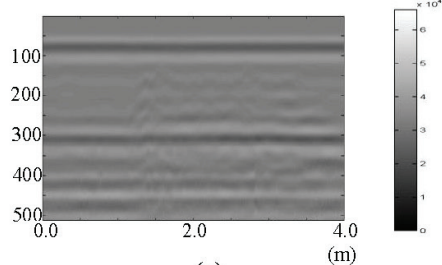

(c)
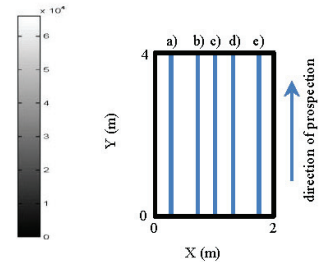

In Figures 22 and 23, the observation of anomalies is deemed very complex. Only a small perturbation from (a) to (d) in Figure 23 can be observed.

In Figures 24 and 25, we present the fieldwork preprocessed images corresponding to the horizontal and vertical profiles, respectively.

Figure 24. Horizontal profiles: pre-processed images. (a-h) are profiles sf1 a sf8, respectively.

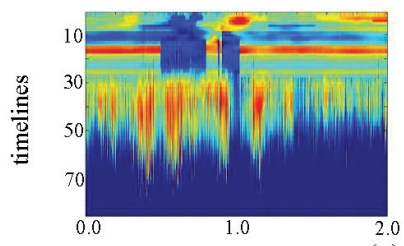

(a)

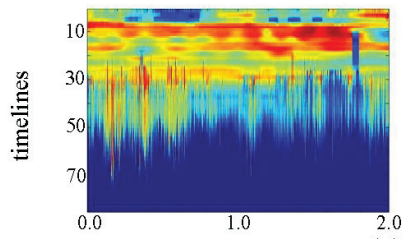

(d)

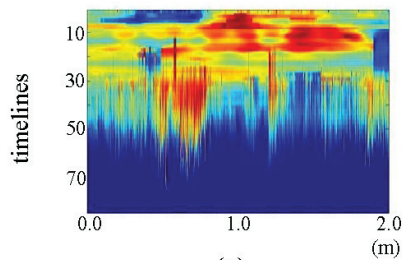

(g)

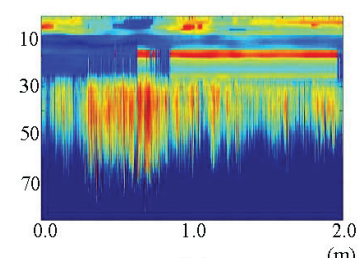

(b)

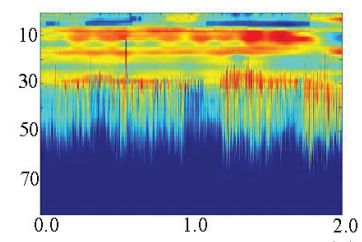

(e)

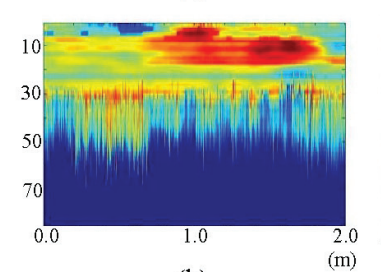

(h)

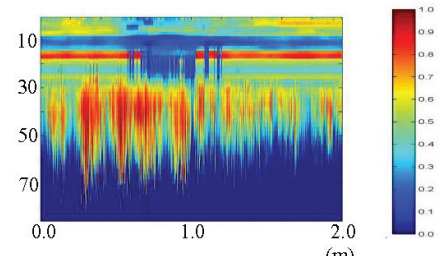

(c)

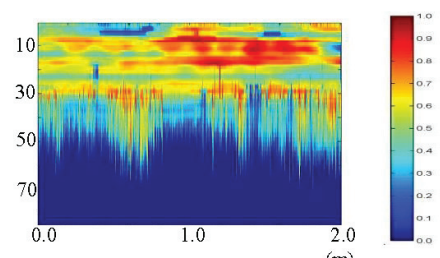

(f)

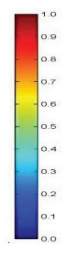

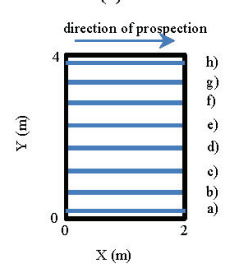

$X$ (m) 
Figure 25. Vertical profiles: pre-processed images. (a-h) are profiles sf9 to sf13, respectively.

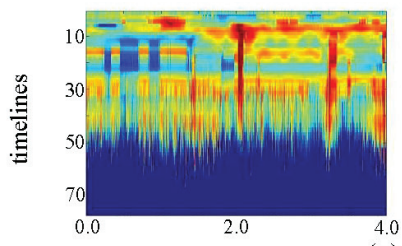

(a)

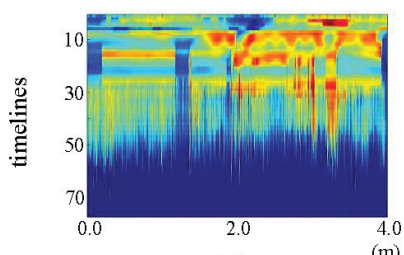

(d)

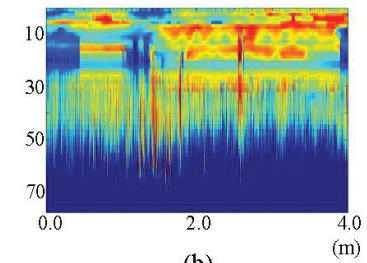

(b)

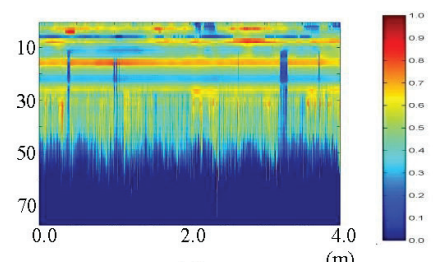

(e)

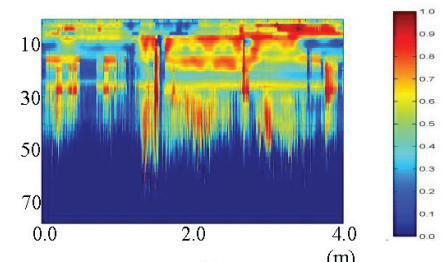

(c)

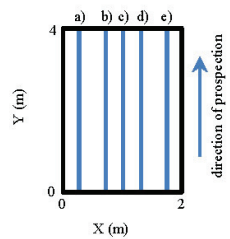

In Figures 24 and 25, the visualization improvement generated with the pre-processing can be clearly seen, making it possible to identify the elliptical shape of the pipe in the horizontal profiles (Figure 24), centered approximately on the position (1 m, five samples). Moreover, in this same figure, in pictures (d) to (h), a structure can be seen that starts at the bottom right of the ellipse corresponding to the pipe. This structure gains strength and is clearly demarcated as an ellipse in the direction (d) to (h). In Figure 25, two vertical structures at $1.5 \mathrm{~m}$ and $3.0 \mathrm{~m}$, in sample 10, are also seen. These structures start from image (a), intensify towards image (c) and reduce their intensity in d), until finally disappearing in (e). This effect corresponds to the convolution observed in Figure 7.

Figure 26 shows the conjugation of these interpretations as 3D models, both for the pipe and likely leaks.

Figure 26. Interpretation from pre-processed images: 3D model. (a) azimuthal view; (b) lateral view \#1; (c) lateral view \#2.

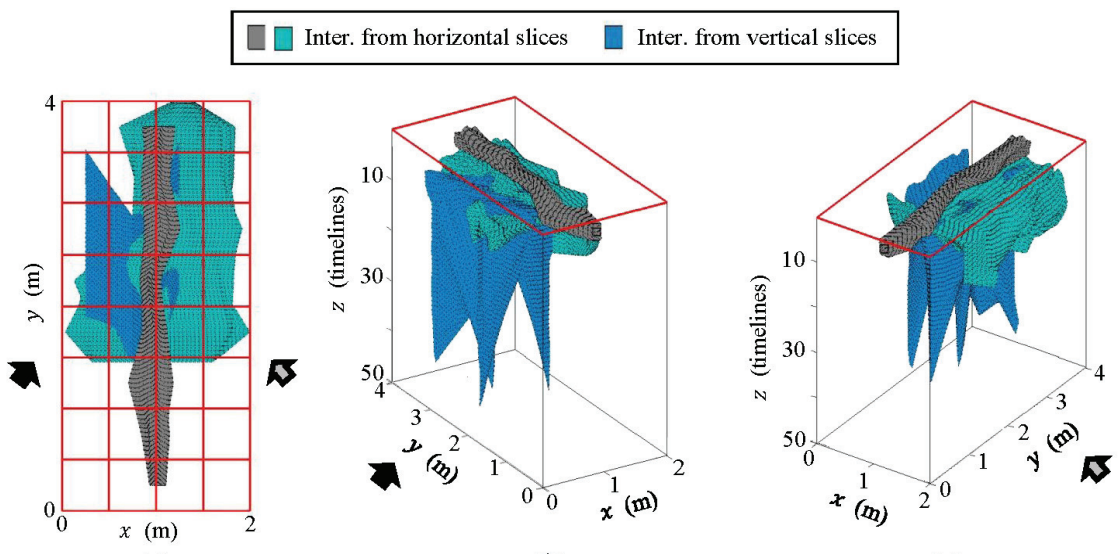

(a)

(b)

(c) 
In Figure 26, water spots generated by the water leak in the pipe can be observed. These spots coincide with the turbulence points detected with the geophone.

\section{Conclusions}

By reducing the amount of water leaked, WDS managers can reduce the amount of money and energy wasted on producing or purchasing water. The use of various types of smart sensors to gather data and the application of advanced analytics, such as pattern detection, could provide valuable information on the location of leaks in the network. GPR, among other various sensors, can help detect potential leaks and abnormalities within WDSs.

In this work, two GPR tests have been conducted in laboratory conditions. One test employed a PVC pipe with no water inside and, obviously, without leaks, and the other test used the same pipe with water inside and some leakage. Additionally, we have conducted a field test in a leaking WDS. The different analyses show the difficulty of making interpretations from raw images. Indeed, it follows from these tests that introducing water into the system causes significant (numeric) differences that are not easily discernible from typical interpretations of the raw data obtained.

Moreover, this work shows that the application of appropriate preprocessing methodologies facilitates the visualization of features that are not reflected in the raw images. This is true both in the case of laboratory testing and in field cases. Consequently, we argue that the pre-processing used in this work facilitates the work of interpretation for non-highly qualified personnel in the use of GPR.

Additionally, it should be mentioned that in this type of pre-process, characteristics are obtained that are quantifiable (numerically). These characteristics can be the basis for further processing for the automatic classification of leaks in WDS.

Moreover, from these tests, we can conclude that the interpretations made using GPR are not merely subjective results. This is supported by the fact that there is consistency for all the results in 3D models between what is captured by GPR and what is expected. Thus, the reconstruction of 3D models from GPR analyses facilitates the interpretation of results.

\section{Acknowledgments}

This work has been supported by the project, IDAWAS (Intelligent Data Analysis in WAter Systems), DPI2009-11591, of the Dirección General de Investigación of the Ministerio de Ciencia e Innovación of Spain, ACOMP (Acción Complementaria)/2011/188, of the Conselleria d'Educació of the Generalitat Valenciana and the FPI (Formación de Personal Investigador)-UPV (Universitat Politècnica de València) scholarship granted to the first author by the Programa de Ayudas de Investigación y Desarrollo (PAID) of the Universitat Politècnica de València. Additionally, we wish to thank the AQUARUM consortium for their help in field data capturing. The use of English in this paper was revised by John Rawlins. 


\section{Conflicts of Interest}

The authors declare no conflicts of interest.

\section{References}

1. GrowingBlue. Available online: http://growingblue.com/wp-content/uploads/2011/04/ Growing-Blue.pdf (accessed on 13 September 2013).

2. Hao, T.; Rogers, C.D.F.; Metje, N.; Chapman, D.N.; Muggleton, J.M.; Foo, K.Y.; Wang, P.; Pennock, S.R.; Atkins, P.R.; Swingler, S.G.; et al. Condition assessment of the buried utility service infrastructure. Tunn. Undergr. Space Technol. 2012, 28, 331-344.

3. Demirci, S.; Yigit, E.; Eskidemir, I.H.; Ozdemir, C. Ground penetrating radar imaging of water leaks from buried pipes based on back-projection method. NDT\&E Int. 2012, 47, 35-42.

4. Dong, L.; Carnalla, S.; Shinozuka, M. Experimental and Analytical Study of Detecting Leakage of Water Pipes Using Ground-Penetrating Radar. In Proceedings of the 9th International Workshop on Remote Sensing for Disaster Response, Standford, CA, USA, 15-16 September 2011; pp. 1-10.

5. Juliano, T.; Meegoda, J.; Watts, D. Acoustic emission leak detection on a metal pipeline buried in sandy soil. J. Pipeline Syst. Eng. Pract. 2013, 4, 149-155.

6. Bimpas, M.; Amditis, A.; Uzunoglu, N. Detection of water leaks in supply pipes using continuous wave sensor operating at $2.45 \mathrm{GHz}$. J. Appl. Geophys. 2010, 70, 226-236.

7. Crocco, L.; Soldovieri, F.; Millington, T.; Cassidy, N.J. Bistatic tomographic GPR imaging for incipient pipeline leakage evaluation. Prog. Electromagn. Res. 2010, 101, 307-321.

8. Charlton, M.B.; Mulligan, M. Efficient Detection of Mains Water Leaks Using Ground Penetrating Radar. In Proceedings of the Subsurface and Sensing Technologies and Applications III, San Diego, CA, USA, 30 July-1 August 2001; pp. 375-386.

9. Stampolidis, A.; Soupios, P.; Vallianatos, F. Detection of Leaks in Buried Plastic Water Distribution Pipes in Urban Places-a Case Study. In Proceedings of the 2nd International Workshop on Advanced GPR, Delft, The Netherlands, 14-16 May 2003; pp. 120-124.

10. Hunaidi, O.; Giamou, P. Ground-Penetrating Radar for Detection of Leaks in Buried Plastic Water Distribution Pipes. In Proceedings of the 7th International Conference on Ground Penetrating Radar, Lawrence, KS, USA, 27-30 May 1998; pp. 783-786.

11. Liu, G.; Jia, Y.; Liu, H.; Qiu, H.; Qiu, D.; Shan, H. A case study to detect the leakage of underground pressureless cement sewage water pipe using GPR, electrical and chemical data. Environ. Sci. Technol. 2002, 36, 1077-1085.

12. Hyun, S.-Y.; Jo, Y.-S.; Oh, H.-C.; Kim, S.-Y.; Kim, Y.-S. The laboratory scaled-down model of a ground-penetrating radar for leak detection of water pipes. Meas. Sci. Technol. 2007, 18, 2791-2799.

13. Hasan, A.E. The Use of Ground Penetrating Radar with a Frequency $1 \mathrm{GHz}$ to Detect Water Leaks from Pipelines. In Proceedings of the 16th International Water Technology Conference (IWTC16), Istanbul, Turkey, 7-10 May 2012; pp. 1-6. 
14. Tavera, M. Aplicación de Georadar Para la Mejora del Rendimiento de una Red Hídrica. Master's Thesis, Polytechnic University of Valencia, Valencia, Spain, May 2008.

15. Ayala-Cabrera, D.; Izquierdo, J.; Montalvo, I.; Pérez-García, R. Water supply system component evaluation from GPR radargrams using a multi-agent approach. Math. Comput. Model. 2013, 57, 1927-1932.

16. Shoham, K.; Leyton-Brown, K. Multiagent Systems: Algorithmic, Game-Theoretic and Logical Foundations; Cambridge University Press: Cambridge, MA, USA, 2009.

17. Ayala-Cabrera, D.; Herrera, M.; Izquierdo, J.; Pérez-García, R. Location of buried plastic pipes using multi-agent support based on GPR images. J. Appl. Geophys. 2011, 75, 679-686.

18. Herrera, M.; Izquierdo, J.; Pérez-García, R.; Ayala-Cabrera, D. Water Supply Clusters by Multi-Agent based Approach. In Proceedings of the Water Distribution System Analysis 2010, Tucson, AZ, USA, 12-15 September 2010; pp. 861-869.

19. Russell, B.C.; Sivic, J.; Ponce, J.; Dessales, H. Automatic Alignment of Paintings and Photographs Depicting a 3D Scene. In Proceedings of the 3rd International IEEE Workshop on 3D Representation for Recognition (3dRR-11), Associated with ICCV 2011, Barcelona, Spain, 7 November 2011; pp. 1-8. 


\title{
A Proposed Scalable Design and Simulation of Wireless Sensor Network-Based Long-Distance Water Pipeline Leakage Monitoring System
}

\author{
Abdulaziz S. Almazyad, Yasser M. Seddiq, Ahmed M. Alotaibi, Ahmed Y. Al-Nasheri, \\ Mohammed S. BenSaleh, Abdulfattah M. Obeid and Syed Manzoor Qasim
}

\begin{abstract}
Anomalies such as leakage and bursts in water pipelines have severe consequences for the environment and the economy. To ensure the reliability of water pipelines, they must be monitored effectively. Wireless Sensor Networks (WSNs) have emerged as an effective technology for monitoring critical infrastructure such as water, oil and gas pipelines. In this paper, we present a scalable design and simulation of a water pipeline leakage monitoring system using Radio Frequency IDentification (RFID) and WSN technology. The proposed design targets long-distance aboveground water pipelines that have special considerations for maintenance, energy consumption and cost. The design is based on deploying a group of mobile wireless sensor nodes inside the pipeline and allowing them to work cooperatively according to a prescheduled order. Under this mechanism, only one node is active at a time, while the other nodes are sleeping. The node whose turn is next wakes up according to one of three wakeup techniques: location-based, time-based and interrupt-driven. In this paper, mathematical models are derived for each technique to estimate the corresponding energy consumption and memory size requirements. The proposed equations are analyzed and the results are validated using simulation.
\end{abstract}

Reprinted from Sensors. Cite as: Almazyad, A.S.; Seddiq, Y.M.; Alotaibi, A.M.; Al-Nasheri, A.Y.; BenSaleh, M.S.; Obeid, A.M.; Qasim, S.M. A Proposed Scalable Design and Simulation of Wireless Sensor Network-Based Long-Distance Water Pipeline Leakage Monitoring System. Sensors 2014, 14, 3557-3577.

\section{Introduction}

The worldwide losses of water due to the distribution network leakage is estimated at 48.6 billion $\mathrm{m}^{3}$, thus causing a monetary loss of approximately 14.6 billion US dollars per year, according to a World Bank study [1]. Water is an important and limited resource, hence it is necessary to protect and use the water utilities efficiently. Water leakage is considered to be one of the largest and most serious challenges. It is expected to intensify over time, given the scarcity of available traditional water resources and the enormous costs of providing fresh potable water from non-traditional sources such as desalination plants. Long-distance water pipelines have become an indispensable part of such infrastructure. Active monitoring and inspection is required to maintain the health of the pipelines [2,3]. A pipeline monitoring system has a long list of tasks to accomplish. In addition to detecting and localizing leakage and bursts, these tasks include measuring pipe cross-section and wall thickness and monitoring fluid purity and flow speed $[4,5]$.

Pipeline monitoring tasks become more challenging when applied to long-distance water pipelines covering thousands of kilometers. Several issues should be considered, such as the 
difficulty of the maintenance of the static components and efficiency of memory usage and energy consumption. Wireless sensor networks (WSNs) provide an efficient way to address these issues. To the best of our knowledge, the problem of monitoring long distance water pipelines using WSNs has not been properly addressed in the published literature, despite its requirement in the practical field.

Different type of sensors, such as temperature sensor, pressure sensor, acoustic sensor, flow sensor, and $\mathrm{pH}$ sensor are typically used for water pipeline monitoring. These sensors generate appropriate electrical signals based on the sensed phenomena. Generally, monitored parameters include temperature, humidity, flow and pressure. Selecting an appropriate sensor or sensing technique depends on many aspects such as the pipeline material and environment (aboveground or underground).

A typical WSN node consists of a sensing subsystem, a processing subsystem, a communication subsystem and a power supply subsystem. The processing subsystem which mainly comprises of microcontroller and memory processes and stores the sensor data respectively. The RF transceiver, which is an important part of communication subsystem receives commands from a central computer and transmits the processed data to that computer. The power for each WSN node is derived from a battery or an energy harvesting (scavenging) device.

In this paper, a scalable design and simulation of a long-distance above ground water pipeline leakage monitoring system using WSN is proposed. The challenges of difficult maintenance, efficient memory usage and low energy consumption are considered. The design is based on deploying mobile sensor nodes that are driven by the water current. A multi-node model is adopted in order to make the design scalable for various distances, memory sizes and battery lifetimes. Among the deployed nodes, one should be in-duty while the others are sleeping. At a certain stage, the active node turns itself off after it hands over the task to another node. Task handover takes place using the following three methods: location-based, time-based and interrupt-driven. Localization is done using Radio Frequency IDentification (RFID) tags that are placed at fixed positions outside the pipeline. Mathematical models are also derived to estimate the energy consumption and the memory usage of the proposed design.

The rest of the paper is organized as follows: Section 2 presents the related work. The proposed design is described in Section 3. The mathematical models are discussed and analyzed in Section 4. Section 5 presents the simulation results of the proposed system. The results are validated through Matlab simulation in Section 6. Finally, conclusions and recommendations for future work are discussed in Section 7.

\section{Related Work}

A number of WSN-based solutions for pipeline monitoring have been proposed in the literature [2,3]. Jawhar et al. presented an initial framework for using linear WSNs for oil, gas, and water pipeline monitoring applications [6]. PipeNet is one such system which is used for automated detection, localization and quantification of leaks, bursts and other anomalies in large diameter bulk water transmission pipelines [7]. Accelerometer sensors are used to measure the vibrations that can result from the presence of cracks in the pipeline. PipeNet provides near real-time operation with few false alarms. A scalable, non-intrusive, autonomous and adaptive water 
monitoring system (NAWMS) is presented in [8]. It detects and locates leakages using low cost wireless vibration sensors that are externally attached to the pipes. It can be used to estimate the water consumption with minimum error.

An autonomous pipeline monitoring system called TriopusNet is presented in [9]. Sensor nodes are automatically released from a centralized repository located at the source of the water pipeline and carried forward by the water flow. The nodes are placed automatically based on a sensor deployment algorithm. Each sensor node includes a motor which allows the three arms to latch onto the pipe's inner surface. This is explained in detail in [9]. Human effort is not required to install and repair sensor nodes in this system.

A fault-tolerant and reliable architecture based on an integrated wired and wireless sensor network for monitoring aboveground pipeline infrastructures is presented in [10]. SPAMMS is a low-cost, scalable, customizable and autonomous sensor-based system which is presented in [11]. This system combines sensing technology with robot agent-based technology to provide active and corrective monitoring and maintenance of the pipelines. SPAMMS combines RFID systems with mobile sensors and autonomous robots to monitor pipelines. Different pipeline monitoring techniques are compared and discussed in this paper [11].

Underground pipelines are mostly preferred to transport water from remote locations. This provides the safest way to transport water, but at the cost of extreme environmental conditions under the ground which may cause leakage on the pipelines [12-14]. A low-cost magnetic induction waveguide-based WSN technique for underground pipeline monitoring (MISE-PIPE) is presented in [12]. In MISE-PIPE, two type of sensors are used, one placed inside and the other placed outside the pipeline. Both internal and external sensors provide sufficient data for detecting and localizing the leakage in the pipeline. The authors claim that this technique can provide accurate real-time leakage detection and improved lifetime for the underground pipelines.

PipeTECT, an intelligent and scalable WSN system for real-time nondestructive monitoring of underground water pipelines is discussed in [15]. MEMS accelerometers on the pipe surface are employed to measure vibrations in order to determine the change in the water pressure caused by pipe rupture and thus localize the leakage. However, it faces some challenges such as reliable long-range communication, precise time synchronization, power management and effective bandwidth usage [15]. The PipeTECT system was further improved by adding new modules at the sensing and data aggregation unit which reduced the total energy consumption significantly [16].

\section{Proposed Design}

This work proposes a non-real-time leakage monitoring system for long-distance water pipelines. A mobile sensor node is allowed to move with the water current from the pipeline source down to the sink where the node is collected and its memory content is copied to a computer. This data contains all the sensor and location readings that are taken by the node throughout its long trip inside the pipeline. The node observation is subjected to offline analysis to locate the leakage.

A node records its location based on its exposure to signals of RFID tags that are placed in fixed position outside the pipeline surface. The number of tags used is inversely proportional to the 
distance between tags $(\Delta d)$. If the total pipeline distance is $D$, then the number of RFID tags required for the whole system $(M)$ can be calculated as follows:

$$
M=\left[\frac{D}{\Delta d}\right]
$$

The use of active RFID tags, which are battery-operated, enables their signal to penetrate through the pipeline walls. Some active RFID tags today are able to transmit up to $200 \mathrm{~m}$ in free space (e.g., the ZT-50) [17]. Thus, tag signal penetration through few tens of centimeters of the pipe wall would be possible provided that the pipeline material allows signal propagation. Nevertheless, this assumption is not valid for strongly isolating materials such as steel.

Basically, active RFID tags are battery-operated, which implies the need for replacing their batteries from time to time. That would not be easy when considering long-distance pipelines that pass through rural and difficult to approach areas. For that reason, solar cells can be used as a renewable power source for the active RFID tags. A general illustration of the proposed design is shown in Figure 1.

Figure 1. (a) Proposed design components; (b) Loose independent nodes; (c) Nodes connected in series using wires (For interrupt-driven method).

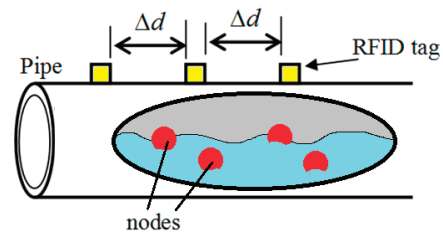

(a)

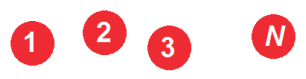

(b)

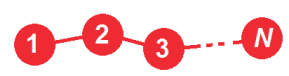

(c)

In order to add scalability and efficiency to the system and to simplify the node design, a multi-node model is adopted in this design. That is, during the trip period $(T)$ in the pipeline, a group of $N$ nodes, where $N>1$, are deployed and allowed to move with the water current. These nodes work cooperatively to perform the monitoring tasks by allowing only one node to be in duty for a certain interval $T_{A}$ while the other nodes are inactive. The active node gets busy sensing and localizing leakages before it cuts off after a period of $T_{A}$ since it commenced its mission. That active period $\left(T_{A}\right)$ is determined as follows:

$$
T_{A}=\frac{T}{N}
$$

The inactive nodes are either totally off, if they already finished duty, or sleeping, if they are awaiting their turn to start duty. Duty handover from one node to another takes place using one of the following three methods: location-based, time-based and interrupt-driven. 
In the location-based method, the sleeping nodes keep locating themselves while sleeping. Each node knows where it should commence duty and hence wakes up. In contrast, in the second method, which is time-based, the sleeping nodes have to keep their timer on during the sleep period. When the appropriate time of commencing duty comes, the node wakes up. Since, nodes float independent of each other, racing between nodes may occur and it is possible that a node that is about to wake up is way ahead of the node that is currently in duty. In this situation, there will be a pipeline segment, which might have leakage and not monitored by either of the two nodes. A possible solution could be a time overlap between them to reduce the chance of having that problem. Certainly, this redundancy will increase the energy consumption. A more detailed analytical proof of pipeline coverage by the three techniques is provided in the Appendix section.

The third method involves an interrupt-driven wakeup. When using this method, sleeping nodes neither locate nor do they count time. Rather, a sleeping node waits for an interrupt signal from the active node via a wire connecting them. Therefore, when using the interrupt-driven wakeup method, the nodes must be connected in series using wires in a chain as shown in Figure 1c. The series connection may cause a reliability problem. That is, if any node breaks down, all the subsequent nodes will be out of service. Perhaps the use of a wire can be avoided by reusing nodes and having them go into sleep mode while waiting for an interrupt signal. With proper packaging and mechanical design, the node can be made floatable [18].

Regardless of the duty handover method that is used, when an in-duty node finishes its task, it cuts off and it does not perform any activity until it reaches the pipeline sink. The transition from sleep to active modes and from active to cut-off mode is illustrated in Figure 2. Table 1 summarizes the activities during the sleep and active modes.

Each node should be equipped with components that enable it to perform its job efficiently. For localization, a node uses an RFID tag reader that can acquire the IDs of the active tags every time the node passes under an active tag. Since, the main purpose of deploying the node is to sense, the presence of a sensor (e.g., pressure or velocity) is necessary. The RFID reader and the sensor are controlled by a low-energy microcontroller. A general block diagram of the sensor node is shown in Figure 3. 
Figure 2. Sleep-active modes of the proposed design.

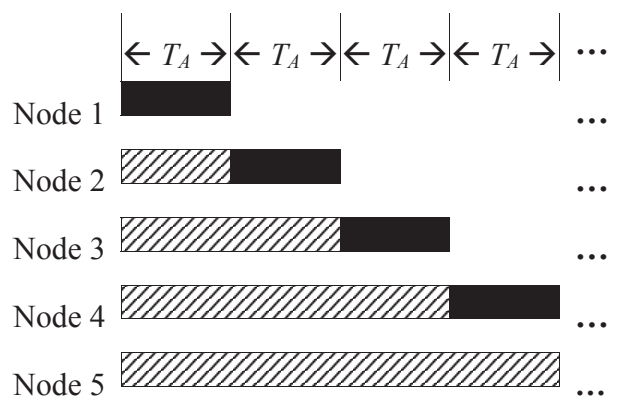

$\bullet \bullet \bullet$ etc.

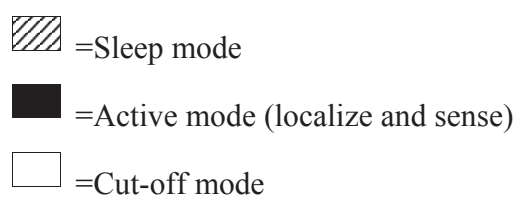

Table 1. Major activities during the sleep and active modes.

\begin{tabular}{|c|c|c|c|c|}
\hline & \multicolumn{3}{|c|}{ Major activities during sleep mode } & \multirow{2}{*}{$\begin{array}{l}\text { Major activities during } \\
\text { active mode }\end{array}$} \\
\hline & Location-based & Time-based & Interrupt-driven & \\
\hline Sensor & Off & Off & Off & $\begin{array}{l}\text { Busy sensing the pipeline } \\
\text { environment (e.g., pressure } \\
\text { sensing) }\end{array}$ \\
\hline RFID reader & $\begin{array}{l}\text { Communicating } \\
\text { with RFID tags }\end{array}$ & Off & Off & $\begin{array}{l}\text { Communicating with } \\
\text { RFID tags }\end{array}$ \\
\hline Microcontroller & $\begin{array}{l}\text { Busy processing } \\
\text { the RFID } \\
\text { information for the } \\
\text { purpose of } \\
\text { localization and to } \\
\text { make decision to } \\
\text { wakeup }\end{array}$ & $\begin{array}{l}\text { Busy running the } \\
\text { timer and } \\
\text { processing time } \\
\text { information for } \\
\text { the purpose of } \\
\text { making decision } \\
\text { to wakeup }\end{array}$ & Off & $\begin{array}{l}\text { Busy doing two things: } \\
\text { 1. Collection and storage } \\
\text { of sensor data } \\
\text { 2. Processing of RFID } \\
\text { information for } \\
\text { localization }\end{array}$ \\
\hline
\end{tabular}

Figure 3. General block diagram of sensor node.

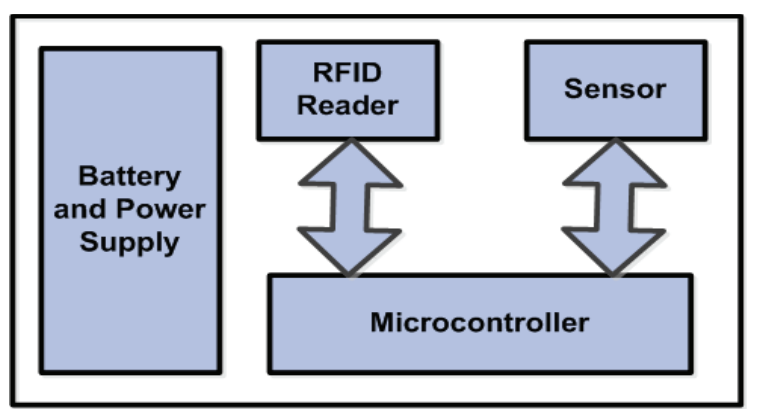




\section{Mathematical Modeling and Analysis}

There are many parameters that affect the energy consumption and the memory usage of each node of the aforementioned design. The key parameters are described in Table 2. For energy consumption, the following relationship is used:

$$
E=P \times T
$$

where, $E$ is the energy consumed during the time period $T$ by a system that consumes power $P$.

Table 2. Design parameters.

\begin{tabular}{lll}
\hline Parameter & Description & Unit \\
\hline$T$ & Total node trip period & seconds \\
$T_{A}$ & The active period of a node & seconds \\
$T_{r d(A)}$ & The period of communication between an RFID reader and a tag & seconds \\
$N$ & Number of deployed nodes & - \\
$E_{n}$ & Energy consumed by the $n^{\text {th }}$ node & Joules \\
$M$ & Total number of RFID tags & - \\
$m$ & Number of RFID tags in a pipeline segment & - \\
$D$ & Total pipeline distance & km \\
$\Delta d$ & Distance between RFID tags & meter \\
$f_{s}$ & Sensor sampling rate & Samples per second \\
$W_{R F I D}, W_{\text {sensor }}$ & Data width of the RFID and the sensor sample respectively & Bytes \\
$P_{r d(i d l e)}, P_{r d(A)}$ & Power consumed by RFID reader in idle & and active modes respectively \\
$P_{C}$ & Average power consumed by the microcontroller & Watts \\
$P_{S}$ & Power consumed by the sensor & Watts \\
\hline
\end{tabular}

When deploying $N$ nodes in the pipeline, the energy consumption of the $n$th node, where $1 \leq n \leq N$, can be estimated as discussed in the following sections.

\subsection{Location-Based Wakeup Method}

To analyze the total energy consumption of the $n^{\text {th }}$ node, the energy consumed during the sleeping mode $E_{n(\text { sleep })}$ and active mode $E_{n(\text { active })}$ should be taken into consideration, i.e.,:

$$
E_{n}=E_{n(\text { sleep })}+E_{n(\text { active })}
$$

In the location-based wakeup method, a sleeping node consumes energy in localizing itself which implies that both the RFID reader and the microcontroller are doing some activities. The $n^{\text {th }}$ node spends a period of $(n-1) T_{A}$ sleeping. Therefore:

$$
E_{n(\text { sleep })}=(n-1)\left(T_{A}\left(P_{r d(\text { idle })}+P_{C}\right)+m T_{r d(A)} P_{r d(A)}\right)
$$

The parameters used in Equation (5) and the subsequent equations are described in Table 2. The term $m T_{r d(A)} P_{r d(A)}$ in Equation (5) refers to the energy consumed by the RFID tag reader when 
communicating with one of the $m$ tags that the node will be exposed to during a period of $T_{A}$. The relationship between $m$ and the total number of the tags $(M)$ is given by Equation (6):

$$
m=\left[\frac{M}{N}\right]
$$

For the active mode of the location-based method, the energy is consumed by all of the node components, i.e., the sensor, the tag reader and the microcontroller. Knowing that the node spends a period of $T_{A}$ in active mode, the following equation can be deduced:

$$
E_{n(\text { active })}=T_{A}\left(P_{r d(\text { idle })}+P_{s}+P_{C}\right)+m T_{r d(A)} P_{r d(A)}
$$

Substituting Equations (5) and (7) in Equation (4) will result in the following Equation (8):

$$
E_{n}=T_{A}\left(n P_{r d(i d l e)}+P_{s}+n P_{C}\right)+n m T_{r d(A)} P_{r d(A)}
$$

\subsection{Time-Based Wakeup Method}

For the time-based wakeup method, the total energy consumed by the $n$th node can be derived by, first, calculating the sleep mode energy as just the energy consumed by the microcontroller (assuming the timer is implemented as a piece of code). Recalling that the $n^{\text {th }}$ node spends a period of $(n-1) T_{A}$ in sleeping mode, the sleep mode energy can be calculated as:

$$
E_{n(\text { sleep })}=(n-1) T_{A} P_{C}
$$

The active mode of the time-based wakeup method is the same as the location-based wakeup method, i.e., it can be calculated using Equation (7). Thus, Equation (10) can be formed by substituting Equations (7) and (9) in Equation (4):

$$
E_{n}=T_{A}\left(P_{s}+P_{r d(i d l e)}+n P_{C}\right)+m T_{r d(A)} P_{r d(A)}
$$

\subsection{Interrupt-Driven Wakeup Method}

Finally, in the interrupt-driven method, a sleeping node does not do any activity while it is in sleep mode. Therefore:

$$
E_{n(\text { sleep })}=0
$$

The active mode energy under the interrupt-driven method is the same as the other two methods and can be determined using Equation (7). Substituting Equations (7) and (11) in Equation (4) will lead to Equation (12):

$$
E_{n}=T_{A}\left(P_{s}+P_{r d(i d l e)}+P_{C}\right)+m T_{r d(A)} P_{r d(A)}
$$

To analyze Equations (8), (10) and (12), several values for the equations variables are assigned according to Table 3 . In addition, Table 4 lists the values of the power consumption of the components that can be used to build the sensor node. 
Table 3. Analysis data $\left(D=400 \mathrm{~km}, T_{R D(A)}=2 \mathrm{~s}\right)$.

\begin{tabular}{cccc}
\hline Parameter & Min. Value & Max. Value & Increment Step \\
\hline$T$ & $10 \mathrm{~h}$ & $70 \mathrm{~h}$ & $20 \mathrm{~h}$ \\
$N$ & 1 node & 60 nodes & 5 nodes \\
$\Delta d$ & $10 \mathrm{~m}$ & $500 \mathrm{~m}$ & $40 \mathrm{~m}$ to increment the minimum value and $50 \mathrm{~m}$ elsewhere \\
\hline
\end{tabular}

Table 4. Sensor node components and typical power consumed.

\begin{tabular}{lcc}
\hline Component & Brand & Power Specs. \\
\hline Pressure sensor & Intersema MS5541C [19] & $18 \mu \mathrm{W}$ \\
Microcontroller & LPC1102 Cortex-M0 [20] & $16.5 \mathrm{~mW}$ \\
RFID reader & Tagsense ZR-232 Active Tag Reader [21] & $9.9 \mu \mathrm{W}$ (idle), 3.3 mW (communicating) \\
RFID tag & Tagsense ZT-50 Active RFID Tag [17] & $9 \mu \mathrm{W}$ (idle), 60 mW (communicating) \\
\hline
\end{tabular}

To derive a mathematical model for calculating the $n$th node memory size, it should be realized that the only two components that write data to memory are the RFID tag reader and the sensor. Moreover, memory is only written to during the active mode, which lasts for a period of $T_{A}$. During that period, the tag reader will communicate to $m$ tags and store their IDs to the memory. If an ID consists of $W_{R F I D}$ bytes, then the total number of bytes that are stored in the memory and belong to the tag reader is as follows:

$$
S_{n(\text { reader })}=m W_{R F I D}
$$

The sensor is the other component that stores data in memory. During the active period $\left(T_{A}\right)$, the sensor performs sensing $f_{s}$ times per second. Each time, a sample of width $W_{\text {sensor }}$ is stored into the memory. The total number of samples during an active period is $f_{S} T_{A}$ samples. And the total number of bytes that the sensor will store into memory is as follows:

$$
S_{n(\text { sensor })}=f_{S} T_{A} W_{\text {sensor }}
$$

When adding Equations (13) and (14), the expression of Equation (15) will be formed.

$$
S_{n}=m W_{R F I D}+f_{s} T_{A} W_{\text {sensor }}
$$

To analyze Equation (15), different values of $N$ are assigned as assumed in Table 3. It is also assumed that the sensor node performs sensing twice a second, i.e., $f_{s}=2$ samples per second. Every time it samples, the sensor sample size $\left(W_{\text {sensor }}\right)$ is assumed to be two bytes long. For the RFID tag, the tag ID is assumed to consist of 16 bytes. The value of the number of RFID tags per pipeline segment $(m)$ can be calculated using Equations (1)-(3), (6), (8), (10), (12).

\section{Results Discussion}

The analysis results for energy consumption are plotted in Figure 4. The $\mathrm{x}$-axis represents the different values of $N$, which is the number of member nodes in a group, e.g., when $n=15$, that refers to a 15-node group deployed in the pipeline. The y-axis refers to the energy consumed by a single node that is a member of an $N$-node group. On each plot, there are two types of curves: solid line and dashed line curves that correspond to the minimum and the maximum distances of 
separation between the RFID tags respectively. That is, the solid line is associated with $\Delta d=10 \mathrm{~m}$ while the dashed line is associated with $\Delta d=500 \mathrm{~m}$. Moreover, each plot contains four curves that represent total trip times of 10, 30, 50 and $70 \mathrm{~h}$. Figure 4 also consists of twelve plots, from $(a)$ to $(l)$, arranged in a matrix of four rows and three columns. The first, second and third columns of the matrix depicts the results of analyzing the location-based, the time-based and the interrupt-driven wakeup methods respectively. In other words, the first, the second and the third columns of the matrix depicts the results of analyzing Equations (8), (10) and (12) respectively. Each row of the matrix focuses on analyzing a specific node within the group using the three wakeup methods. That is, the first, second, third and fourth rows refer to the 1st, the 5th, the 25th and the 50th nodes of the group being analyzed respectively.

It can be seen from Figures $4 \mathrm{a}-\mathrm{c}$, that the energy consumption of the first node is only affected by the number of nodes in the group and the distance between RFID tags, while the wakeup method has totally no effect on it. That is because the first node is not subjected to the sleep mode as it starts in active mode by default. The three wakeup methods differ only in what a node does before coming to duty and according to the way it wakes up. Obviously, none of these two differences are applicable to the first node.

On the other hand, the plots in the second, the third and the fourth rows of Figure 4 show clearly that nodes energy consumption is dependent on the wakeup method that is used. Consider two sets of plots: the set of plots of Figure $4 \mathrm{~d}, \mathrm{~g}$ and $\mathrm{j}$ and the set of plots of Figure $4 \mathrm{e}, \mathrm{h}$ and $\mathrm{k}$. The energy consumption that is depicted in those sets is almost identical. That is because these two sets represent the location-based and the time-based wakeup methods that are both involved in some activity during the sleeping mode. That is, if a node follows the location-based method, it consumes some energy while sleeping to localize itself. Likewise, with the time-based method, some energy is consumed in sleep mode by the node timer. On the other hand, since the interrupt-driven method involves no activity when a node is sleeping, the plots of Figure $4 \mathrm{f}, \mathrm{i}$ and 1 are different than the other two methods. As expected, increasing the number of nodes per group will result in a significant drop in energy consumption. Obviously, that is because of the deep sleep mode that characterizes the interrupt-driven methods. Such significant energy saving can overshadow the reliability problem that is associated with the interrupt driven method that was discussed earlier in Section 3.

It is also clear that the difference between a solid line curve and its dashed line counterpart is small. The reason why the energy consumption in the dashed line curves is always less is because the RFID tags are so far from each other $(\Delta d=500 \mathrm{~m})$. Consequently, the RFID reader does not need to communicate with the tags so often as in the case of solid line curves when $\Delta d=10 \mathrm{~m}$. However, by increasing $\Delta d$, the energy consumption just slightly improves at the expense of significant degradation in localization resolution. Clearly, the insignificant energy saving is not worth that sacrifice in distance resolution.

In general, the energy consumption drops in all the methods, but when a node is scheduled to wake up late (a high value of $n$ ), that drop becomes less sharp. However, that is not true with the interrupt-driven method, which maintains the significant reduction even with nodes that wake up too late. The justification for this is that when considering the time-based or the location-based methods, a sleeping node still consumes energy to count time and locate itself, which are marginal activities that are not within the core duties of the node. 

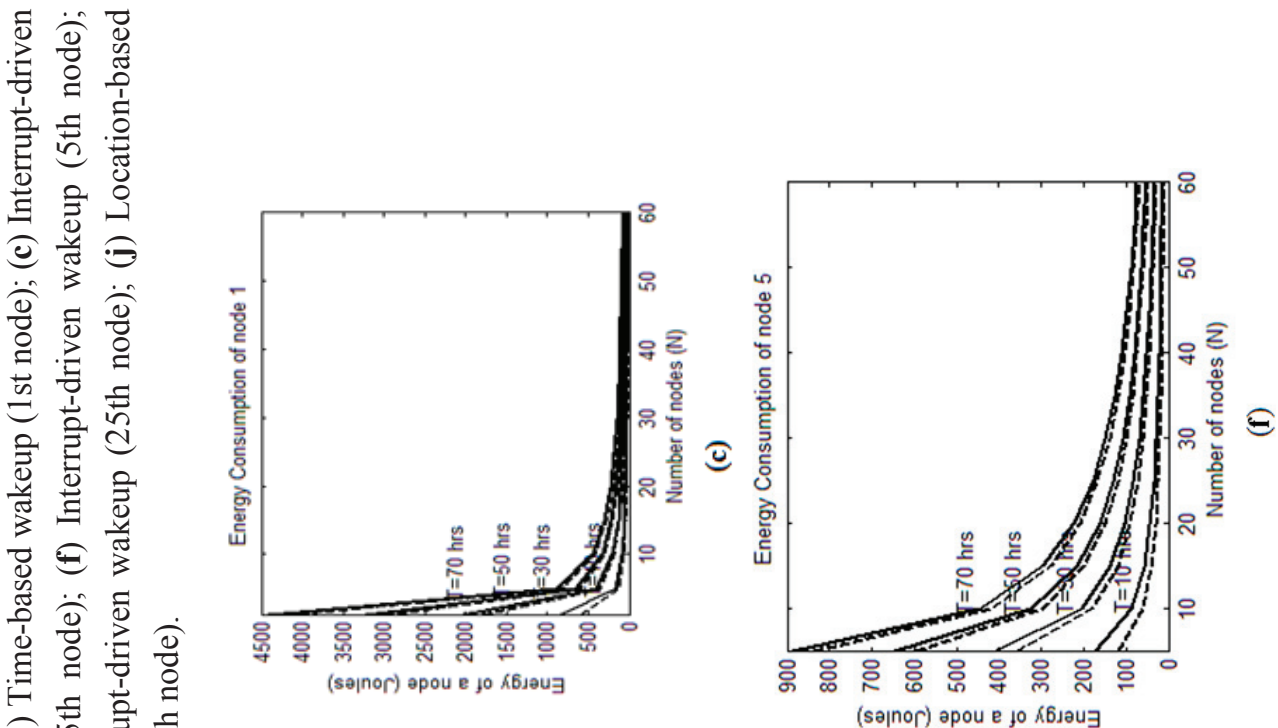

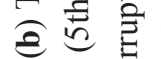
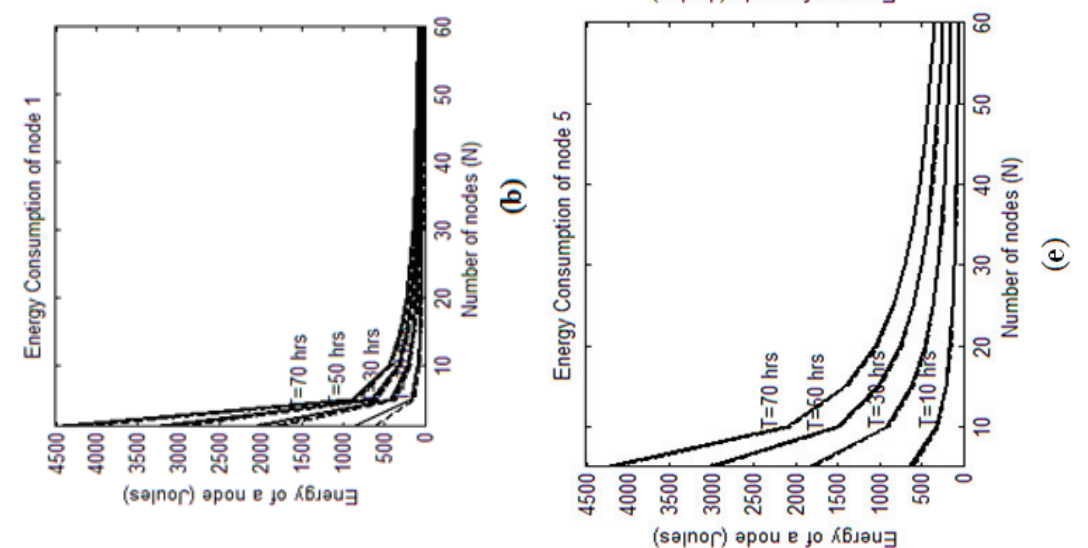

(ิ)
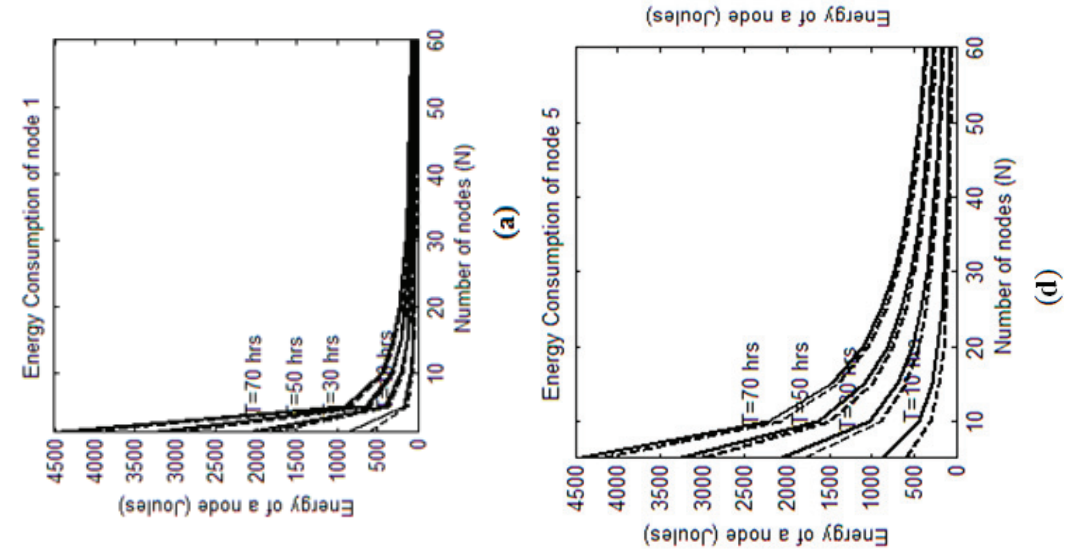

武

(sonnop) apou e to h6.au (sejnor) әpou e to ḱ6ıəuヨ

$+2$

产

की 

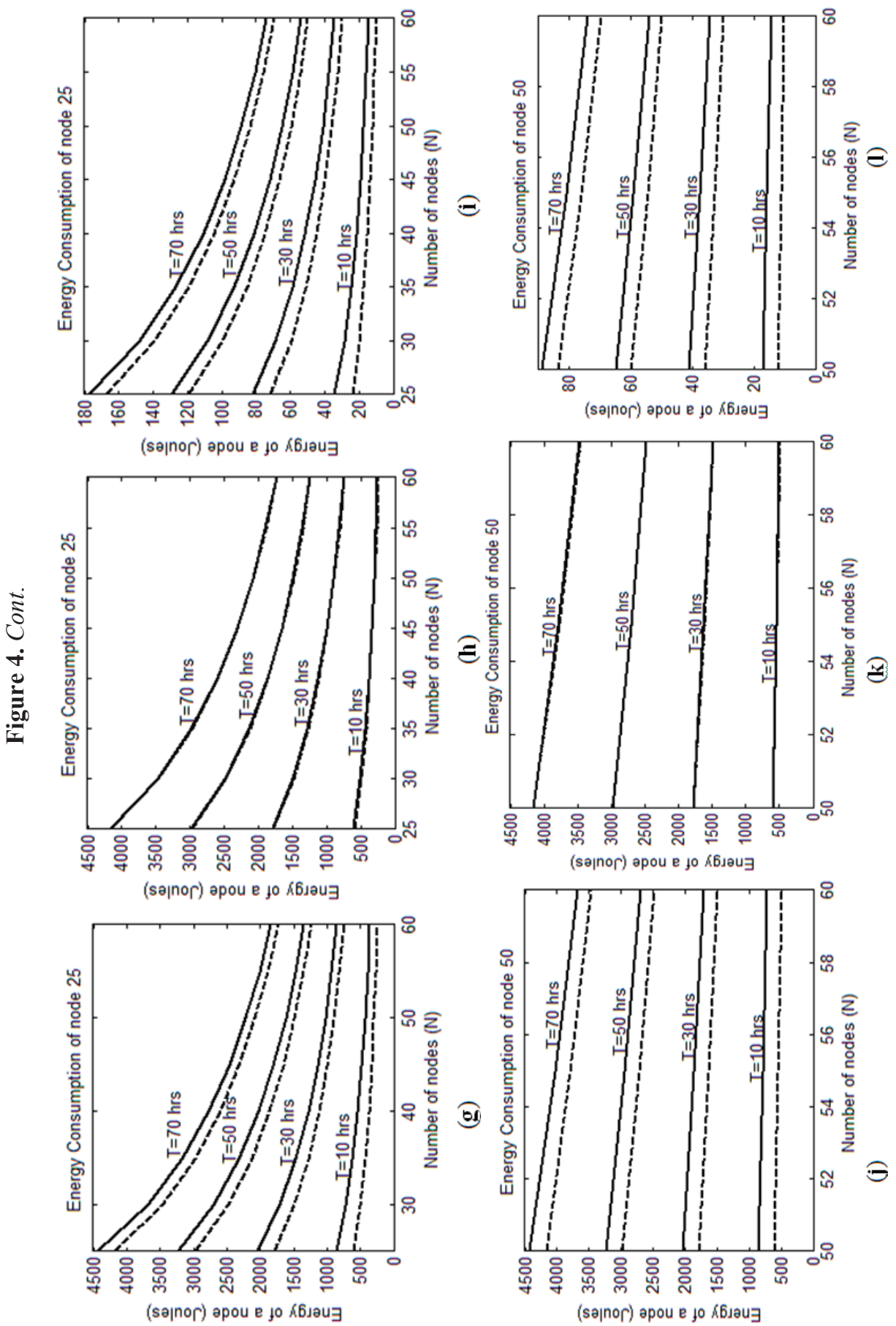
The longer the sleep period is, the more those marginal activities are performed. In contrast, a node that works under the interrupt-driven method does not suffer from those marginal activities since it is in deep sleep mode and doing absolutely nothing when awaiting its turn to wakeup. The existence of the marginal activities in the location-based and the time-based methods introduces limitations to the design scalability when choosing these two methods. However, the interrupt-driven method is scalable to any number of nodes and any trip period.

The second part of this analysis is concerned about the nodes memory size. The relationship between memory size and the other parameters is governed by Equation (15). The analysis results are depicted in Figure 5. The x-axis refers to total number of nodes per group while the y-axis refers to the percentage memory utilization for each node. Figure 5a shows that the memory size of a node drops sharply after adopting the multi-node model. The chart of Figure $5 \mathrm{~b}$ shows the effect of increasing the separation between RFID tags on the node memory usage. When the value of $\Delta d$ is increased from $10 \mathrm{~m}$ to $500 \mathrm{~m}$, the memory size is dropped to almost $50 \%$. This reduction is significant and in case of running short of memory, sacrificing the localization resolution is worth the gain in memory saving.

Figure 5. (a) Node memory utilization $(T=30 \mathrm{~h}, \Delta d=10 \mathrm{~m})$; (b) Node memory utilization (When $N>1$ ) for $T=50 \mathrm{~h}$.

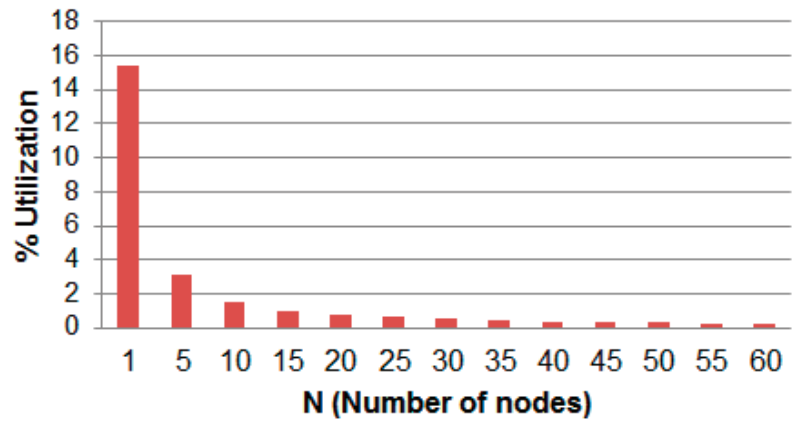

(a)

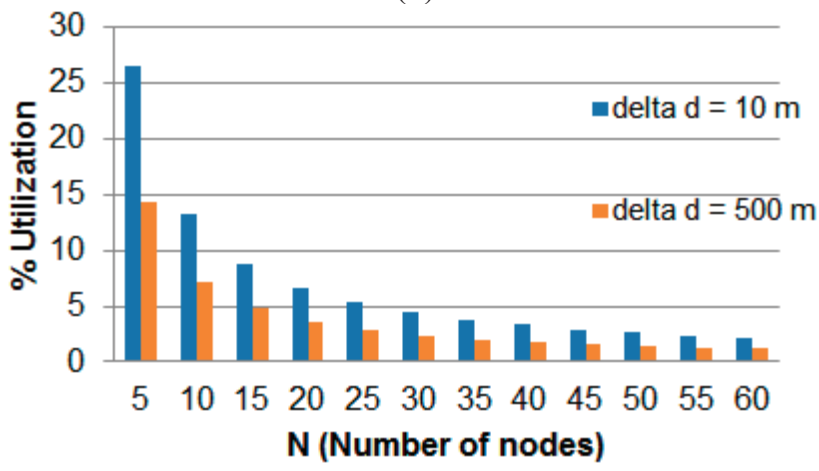

(b)

\section{Model Validation Using Simulation}

An event-driven simulation was carried out using Matlab to validate the analytical models formulated in Equations (8), (10) and (12) and the results presented in Figure 4. The design parameters 
were tuned according to Table 3. For each combination of parameter values, the simulated energy results $\left(E_{\text {sim }}\right)$ were compared with the corresponding analytical results obtained from the proposed equations $\left(E_{e q}\right)$.

The simulation is triggered by some events based on whether the node is in active or sleep mode. While in active mode, the simulation is triggered by an event when the node is exposed to an RFID tag. After every event, the energy is calculated for each component contributing in reading the RFID communication, namely, the microcontroller and the RFID reader. Also, the energy of the pressure sensor is calculated considering operation time throughout the active mode. While in sleep mode, the simulation is triggered by RFID tag exposure as well. However, the energy calculation differs based on the three techniques listed in Table 1. For this scenario, both the simulated and the analytical energy results were compared and found to be identical with zero error.

The simulation was repeated with less event resolution. That is, instead of triggering the simulation by every RFID tag exposure, the simulation is triggered every $T_{A}$ seconds, where $T_{A}$ is a node active period calculated using Equation (2). During the time period $\left(T_{A}\right)$, a node is exposed to $m$ RFID tags as described in Table 2. Under this scenario, the results were found to be almost identical, with a very small error. The error was calculated by taking the absolute value of the difference of the simulated and analytical results, and as the percentage of the absolute error relative to the simulation results. That is:

$$
\begin{gathered}
\text { AbsoluteError }=\left|E_{\text {sim }}-E_{e q}\right| \\
\text { RelativeError }=\frac{\left|E_{\text {sim }}-E_{e q}\right|}{E_{\text {sim }}} \times 100 \%
\end{gathered}
$$

Table 5 summarizes the comparison results of the whole experiment using the three wakeup techniques. The histogram results of the error analysis are also shown in Figure 6.

\section{Conclusions}

Water pipeline networks are considered to be an important asset which requires active monitoring and inspection systems for maintenance. Monitoring long distance water pipeline for leakages, bursts and other anomalies is a challenging task which requires energy-efficient, scalable and robust mechanisms. WSN is one such technology which provides robust solutions to these problems. In this paper, a scalable design and simulation of a non-real-time RFID-WSN-based long-distance aboveground water pipeline leakage monitoring system is presented. The system is based on deploying multiple mobile sensor nodes such that only one node is active for specific period of time. While a node is active, the other nodes are in sleep mode. Sleeping nodes wakeup using three different techniques: location-based, time-based and interrupt-driven. After finishing duty, a node cuts off until the end of its trip. Mathematical models for the energy consumption and the memory usage are proposed, analyzed and validated using Matlab simulation. The analysis results shows that energy consumption of the node improve significantly when the work is divided between groups of nodes. Although the energy consumption can be further improved by increasing the distance between RFID tags, that improvement is not significant and it is overweighed by the significant degradation in distance resolution. 


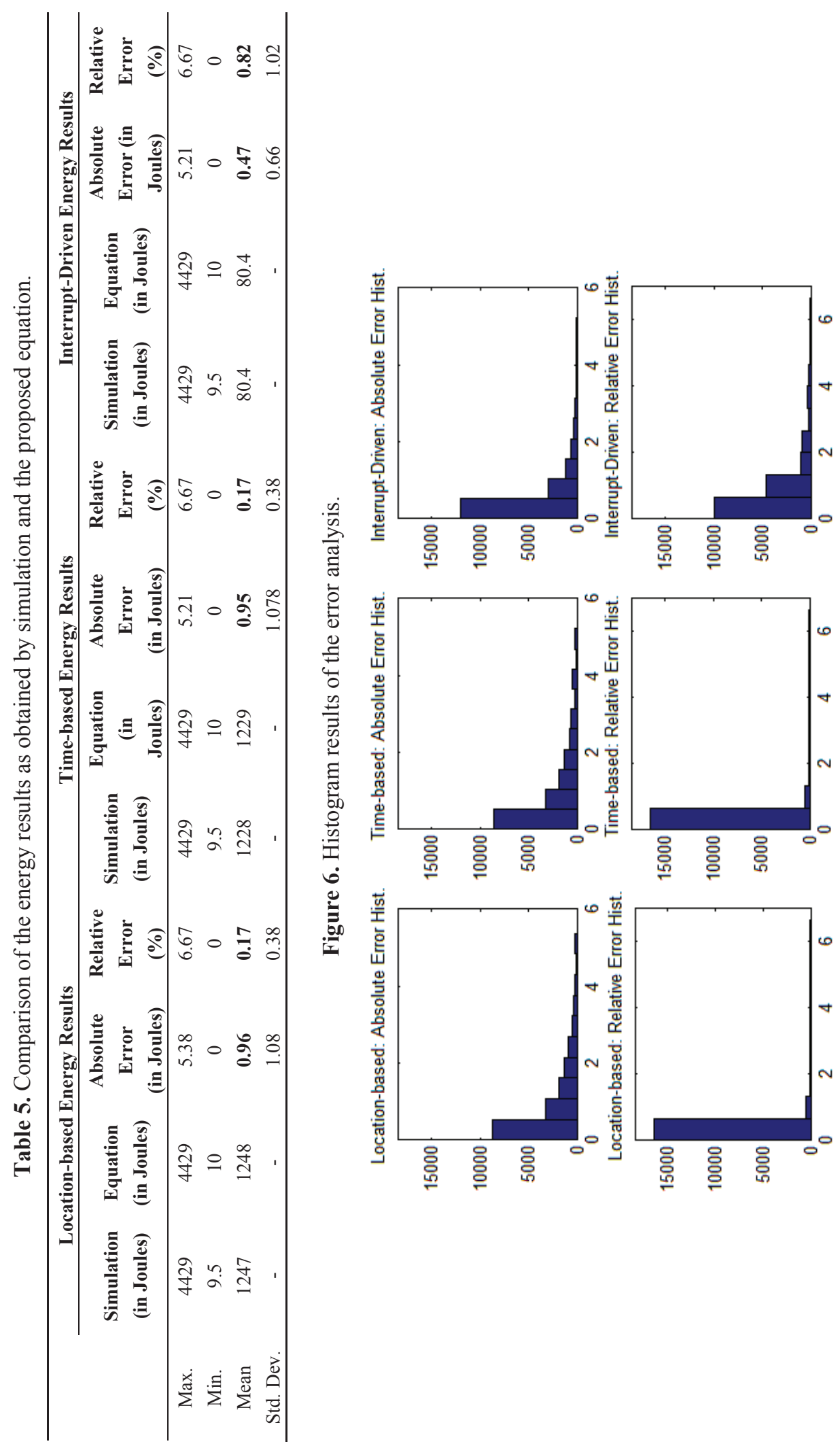


The energy savings that are associated with the location-based and time-based wakeup methods are almost the same. On the other hand, the interrupt-driven method provides a much sharper reduction in energy consumption. This advantage may overshadow the reliability issue of this method due to the fact the nodes are connected in series. One more advantage of the interrupt-driven method is its scalability, even if the number of nodes is high and the trip period is long. In contrast, the other two wakeup methods have limited scalability due to the marginal activities that node need to perform in their sleep mode.

Memory size is also improved when adopting the multi-node model. Unlike the energy saving case, memory saving is also significant when increasing the distance between RFID tags. This analytical work is the first step towards a long term project with objectives of developing an efficient and fault-tolerant monitoring system for a long-distance water pipeline. As a future work, more investigation and development will be carried out to prototype an energy efficient WSN node for water pipeline leakage monitoring system. An experimental testbed based on the resulting node will also be developed and tested using real-world scenarios.

\section{Acknowledgments}

This work is supported by King Abdulaziz City for Science and Technology (KACST) of Saudi Arabia under grant number 31/513 (Project Ejaz).

\section{Author Contributions}

All authors contributed equally to this work.

\section{Conflicts of Interest}

The authors declare no conflict of interest.

\section{Appendix}

\section{Analytical Proof of Pipeline Coverage}

In the location-based technique, missing any part of the pipeline is not possible because the duty handover is always triggered by reaching certain fixed predefined points along the pipeline. Whenever an active node reaches the next handover point it will stop immediately. At the same location, the next node to be in duty will wake up at that point as well. Thus, no matter which of the two nodes are faster than the other, handover will occur with guaranteed coverage for entire pipeline length.

In the interrupt-driven technique, duty handover is triggered by interrupting the sleeping node by the active node. Thus, the handover process implies dependency on the nodes and, hence, coverage is controlled and guaranteed.

For the time-based technique, handover is triggered by internal timers in the active and sleeping nodes. Assuming the timers are synchronized, the two nodes will handover duty at the same time instance. However, knowing that the two nodes can be in undetermined locations, it would be possible 
that the sleeping node (to be active) is ahead of the current active node. Obviously, the location gap between them is not monitored by either of them. A detailed analysis is presented here.

The randomness in the nodes location is due to the randomness in their forward velocity. Nodes, while travelling, are not heading perfectly forward, but there would be some slight drifting towards left or right. Thus, their motions have two components: $x$-component (moving forward) and $y$-component (moving right or left). It is the forward movement that counts in duty handover. Therefore, the forward velocity of a node is not fixed and neither is it deterministic. Thus, it has a random value $v$ ranging between minimum velocity $\left(v_{\min }\right)$ and maximum velocity $\left(v_{\max }\right)$. For the sake of argument, that value is assumed to follow a uniform distribution within $v_{\min }$ and $v_{\max }$ of the density function $f_{V}(v)$ as shown in Figure A1.

Figure A1. The density function of the random variable $v$.

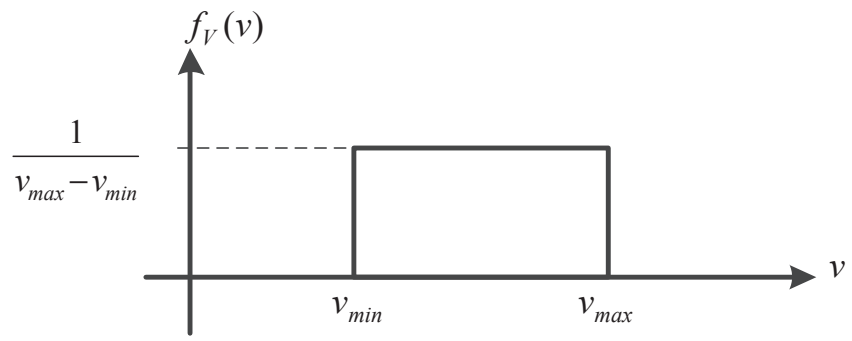

The distance $(d)$ that a node has travelled so far during time $(t)$ can be considered as a random process while $v$ is its random variable. The well-known velocity-time-distance equation can be used to describe that random process. That is:

$$
d(v)=t v
$$

Considering the two nodes involved in the duty handover, lets denote the active node distance as $d_{1}(v)$ while the sleeping node (to be active) as $d_{2}(v)$. If the forward velocities of the first node and the second node are $v_{1}$ and $v_{2}$ respectively, then the following expressions can be used:

$$
\begin{aligned}
& d_{1}\left(v_{1}\right)=t v_{1} \\
& d_{2}\left(v_{2}\right)=t v_{2}
\end{aligned}
$$

The handover that occurs at instance $t$ will not imply missing any part of the pipeline if and only if $d_{2}\left(v_{2}\right) \leq d_{1}\left(v_{1}\right)$ at that instant of time. That is:

$$
\begin{gathered}
d_{2}\left(v_{2}\right)-d_{1}\left(v_{1}\right) \leq 0 \\
t\left(v_{2}-v_{1}\right) \leq 0 \\
t r \leq 0
\end{gathered}
$$

where, $r=\left(v_{2}-v_{1}\right)$ is the relative velocity between the two nodes. Note that $r$ is yet a random variable of density function $f_{R}(r)$. Since the random variable $r$ is the result of the difference between two random variables, $f_{R}(r)$ can be determined using the following relationship [22]: 


$$
f_{R}(r)=\int_{-\infty}^{\infty} f_{V_{1}}\left(r+v_{1}\right) f_{V_{2}}\left(v_{1}\right) d v_{1}
$$

For $r \leq\left(v_{\min }-v_{\max }\right)$, the value of $f_{R}(r)$ is 0 since there will be no overlap between $f_{V_{1}}\left(v_{1}\right)$ and $f_{V_{2}}\left(v_{2}\right)$ (Figure A2a). Likewise, when $r>\left(v_{\max }-v_{\min }\right)$, they do not overlap and, hence, the value of $f_{R}(r)$ is 0 (Figure $\mathrm{A} 2 \mathrm{~d}$ ).

Figure A2. Derivation of the density function of the relative velocity $r$.
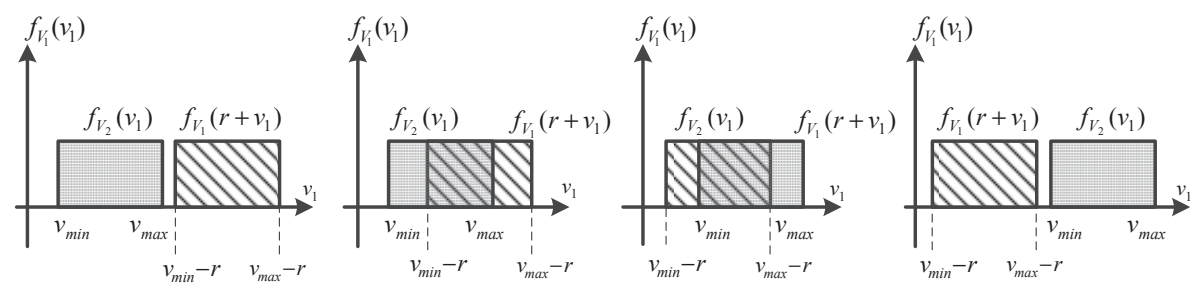

(a) $r \leq\left(v_{\min }-v_{\max }\right)$

(b) $\left(v_{\min }-v_{\max }\right)<r \leq 0$

(c) $0<r \leq\left(v_{\max }-v_{\min }\right)$

(d) $r>\left(v_{\max }-v_{\text {min }}\right)$

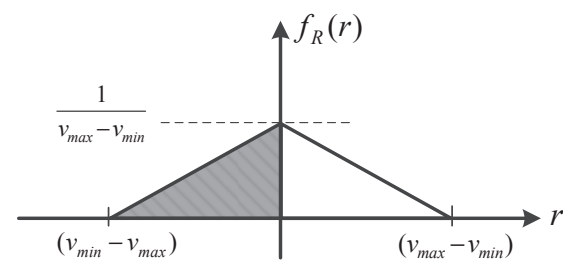

(e) The density function of the random variable $r$

On the other hand, when $\left(v_{\min }-v_{\max }\right)<r \leq 0$, the functions $f_{V_{1}}\left(v_{1}\right)$ and $f_{V_{2}}\left(v_{2}\right)$ overlap from $v_{\min }-r$ to $v_{\max }$ (Figure A2b). That is:

$$
f_{R}(r)=\int_{v_{\min }-r}^{v_{\max }} \frac{1}{\left(v_{\max }-v_{\min }\right)^{2}} d v_{1}=\frac{v_{\max }-v_{\min }+r}{\left(v_{\max }-v_{\min }\right)^{2}} \quad \text { for } v_{\min }-v_{\max }<r \leq 0
$$

Similarly, when $0<r \leq\left(v_{\max }-v_{\min }\right)$, the functions $f_{V_{1}}\left(v_{1}\right)$ and $f_{V_{2}}\left(v_{2}\right)$ overlap from $v_{\min }$ to $v_{\max }-r$ (Figure A2c). That is:

$$
f_{R}(r)=\int_{v_{\min }}^{v_{\max }-r} \frac{1}{\left(v_{\max }-v_{\min }\right)^{2}} d v_{1}=\frac{v_{\max }-v_{\min }-r}{\left(v_{\max }-v_{\min }\right)^{2}} \quad \text { for } 0<r \leq v_{\max }-v_{\min }
$$

In summary, $f_{R}(r)$ can be expressed by the following equation which is also depicted in Figure A2e:

$$
f_{R}(r)=\left\{\begin{array}{cc}
\frac{v_{\text {max }}-v_{\text {min }}+r}{\left(v_{\text {max }}-v_{\text {min }}\right)^{2}} & \text { for } v_{\text {min }}-v_{\text {max }}<r \leq 0 \\
\frac{v_{\text {max }}-v_{\text {min }}-r}{\left(v_{\text {max }}-v_{\text {min }}\right)^{2}} & \text { for } 0<r \leq v_{\text {max }}-v_{\text {min }} \\
0 & \text { otherwise }
\end{array}\right.
$$


The grey region in Figure A2e indicates the interval when $r$ is negative which means that the sleeping node is slower than the active node. Hence, the area under the grey half of the curve is the probability that the handover process does not end up with missing parts of the pipeline unmonitored. That area is equal to 0.5 . On the other hand, the remaining part of the curve is associated with positive values of $r$ meaning that the sleeping node is faster than the active node. Therefore, when handing over duty to the sleeping node, it wakes up in a location that is ahead of the active node, which implies a gap between the two nodes that is not covered by them.

In conclusion, fifty percent of the handover processes are expected to end up with missing some parts of the pipeline. When deploying $N$ nodes in a mission, there will be $N-1$ handovers throughout the pipeline length, fifty percent of which are expected to result in monitoring gaps. That is, $(N-1) / 2$ gaps are expected. Note that, a gap length (like any other distance) is not a function of $N$, but it is a function of velocity and time. Therefore, deploying more nodes in the time-based technique implies increasing the occurrence of handovers and, hence, increasing the total number of potential gaps.

\section{References}

1. Cataldo, A.; Cannazza, G.; Benedetto, E.D.; Giaquinto, N. A new method for detecting leaks in underground water pipelines. IEEE Sens. J. 2012, 12, 1660-1667.

2. BenSaleh, M.S.; Qasim, S.M.; Obeid, A.M.; Garcia-Ortiz, A. A Review on Wireless Sensor Network for Water Pipeline Monitoring Applications. In Proceedings of the International Conference on Collaboration Technologies and Systems (CTS'2013), San Diego, CA, USA, 20-24 May 2013; pp. 128-131.

3. Seddiq, Y.M.; Alotaibi, A.M.; Al-nasheri, A.Y.; Almazyad, A.S.; BenSaleh, M.S.; Qasim, S.M. Evaluation of Energy-Efficient Cooperative Scheme for Wireless Sensor Nodes used in Long Distance Water Pipeline Monitoring Systems. In Proceedings of Fifth International Conference on Computational Intelligence, Communication Systems and Networks (CiCSyn'2013), Madrid, Spain, 5-7 June 2013; pp. 107-111.

4. Jin, Y.W.; Eydgahi, A. Monitoring of Distributed Pipeline Systems by Wireless Sensor Networks. In Proceedings of the 2008 IAJC-IJME International Conference, Nashville, TN, USA, 17-19 November 2008; pp. 1-10.

5. Sinha, D.N. Acoustic sensor for pipeline monitoring, Technical Report, LA-UR-05-6025; Los Alamos National Laboratory: Los Alamos, New Mexico, USA, 20 July 2005; pp. 1-23.

6. Jawhar, I.; Mohamed, N.; Shuaib, K. A Framework for Pipeline Infrastructure Monitoring Using Wireless Sensor Networks. In Proceedings of the Wireless Telecommunications Symposium (WTS'07), Pomona, CA, USA, 26-28 April 2007; pp. 1-7.

7. Stoianov, I.; Nachman, L.; Madden, S.; Tokmouline, T. PIPENET: A Wireless Sensor Network for Pipeline Monitoring. In Proceedings of the Sixth International Conference on Information Processing in Sensor Networks (IPSN'07), Cambridge, MA, USA, 25-27 April 2007; pp. 264-273.

8. Kim, Y.; Schmid, T.; Charbiwala, Z.M.; Friedman, J.; Srivastava, M.B. NAWMS: Nonintrusive Autonomous Water Monitoring System. In Proceedings of the Sixth ACM Conference on Embedded Network Sensor Systems (SenSys'08), Raleigh, NC, USA, 5-7 November 2008; pp. 309-322. 
9. Lai, T.T.-T.; Chen, W.-J.; Li, K.-H.; Huang, P.; Chu, H.-H. TriopusNet: Automating Wireless Sensor Network Deployment and Replacement In Pipeline Monitoring. In Proceedings of the Eleventh International Conference on Information Processing in Sensor Networks (IPSN'12), Beijing, China, 16-20 April 2012; pp. 61-71.

10. Mohamed, N.; Jawhar, I. A Fault Tolerant Wired/Wireless Sensor Network Architecture for Monitoring Pipeline Infrastructures. In Proceedings of the 2nd International Conference on Sensor Technologies and Applications, (SENSORCOMM'08), Cap Esterel, France, 25-31 August 2008; pp. 179-184.

11. Kim, J.; Sharma, G.; Boudriga, N.; Iyengar, S.S. SPAMMS: A Sensor-Based Pipeline Autonomous Monitoring and Maintenance System. In Proceedings of the Second International Conference on Communication Systems and Networks (COMSNETS'10), Bangalore, India, 5-9 January 2010; pp. 1-10.

12. Sun, Z.; Wang, P.; Vuran, M.C.; Al-Rodhaan, M.A.; Al-Dhelaan, A.M.; Akyildiz, I.F. MISE-PIPE: Magnetic induction-based wireless sensor networks for underground pipeline monitoring. Ad Hoc Netw. 2011, 9, 218-227.

13. Akyildiz, I.F.; Stuntebeck, E.P. Wireless underground sensor networks: Research challenges. Ad Hoc Netw. 2006, 4, 669-686.

14. Kadri, A.; Abu-Dayya, A.; Trinchero, D.; Stefanelli, R. Autonomous Sensing for Leakage Detection in Underground Water Pipelines. In Proceedings of the Fifth IEEE International Conference on Sensing Technology, (ICST'11), Palmerston North, New Zealand, 28 November-1 December 2011; pp. 639-643.

15. Shinozuka, M.; Chou, P.H.; Kim, S.; Kim, H.R.; Yoon, E.; Mustafa, H.; Karmakar, D.; Pul, S. Nondestructive Monitoring of a Pipe Network Using a MEMS-based Wireless Network. In Proceedings of the SPIE 7649, Nondestructive Characterization for Composite Materials, Aerospace Engineering, Civil Infrastructure, and Homeland Security, San Diego, CA, USA, 8-11 March 2010; pp. 1-12.

16. Mustafa, H.; Chou, P.H. Embedded Damage Detection in Water Pipelines using Wireless Sensor Networks. In Proceedings of the 14th IEEE International Conference on High Performance Computing and Communication and 9th IEEE International Conference on Embedded Software and Systems (HPCC-ICESS'12), Liverpool, UK, 25-27 June 2012; pp. 1578-1586.

17. ZT-50 Programmable Active RFID Tag with Real-Time Clock. Available online: http://www. tagsense.com/images/stories/products/activetags/ZT-50-short-datasheet-v2.1.pdf (accessed on 30 July 2013).

18. Kim, J.; Lim, J.; Friedman, J.; Lee, U.; Vieira, L.; Rosso, D.; Gerla, M.; Srivastava, M. SewerSnort: A Drifting Sensor for in-situ Sewer Gas Monitoring. In Proceedings of the Sixth Annual IEEE Communications Society Conference on Sensor, Mesh and Ad Hoc Communications and Networks (SECON'09), Rome, Italy, 22-26 June 2009; pp. 1-9.

19. MS5541C-MINIATURE 14 Bar MODULE-Intersema Sensoric SA. Available online: http://pdf1.alldatasheet.com/datasheet-pdf/view/302483/INTERSEMA/MS5541C.html (accessed on 30 July 2013).

20. LPC1102 32-Bit ARM Cortex-M0 Microcontroller. Available online: http://www.nxp.com/ documents/data_sheet/LPC1102.pdf (accessed on 30 July 2013). 
21. ZR-232 and ZR-232 Ultra Embedded Active Tag Reader. Available online: http://www.tagsense. com/images/stories/products/activereaders/ZR-232-Reader-v1-1-Datsheet.pdf (accessed on 30 July 2013).

22. Barkat, M. Signal Detection and Estimation, 2nd ed.; Artech House: Boston, MA, USA, 2005. 


\title{
Energy Efficient Cooperation in Underlay RFID Cognitive Networks for a Water Smart Home
}

\author{
Adnan Nasir, Syed Imtiaz Hussain, Boon-Hee Soong and Khalid Qaraqe
}

\begin{abstract}
Shrinking water resources all over the world and increasing costs of water consumption have prompted water users and distribution companies to come up with water conserving strategies. We have proposed an energy-efficient smart water monitoring application in [1], using low power RFIDs. In the home environment, there exist many primary interferences within a room, such as cell-phones, Bluetooth devices, TV signals, cordless phones and WiFi devices. In order to reduce the interference from our proposed RFID network for these primary devices, we have proposed a cooperating underlay RFID cognitive network for our smart application on water. These underlay RFIDs should strictly adhere to the interference thresholds to work in parallel with the primary wireless devices [2]. This work is an extension of our previous ventures proposed in [2,3], and we enhanced the previous efforts by introducing a new system model and RFIDs. Our proposed scheme is mutually energy efficient and maximizes the signal-to-noise ratio (SNR) for the RFID link, while keeping the interference levels for the primary network below a certain threshold. A closed form expression for the probability density function (pdf) of the SNR at the destination reader/writer and outage probability are derived. Analytical results are verified through simulations. It is also shown that in comparison to non-cognitive selective cooperation, this scheme performs better in the low SNR region for cognitive networks. Moreover, the hidden Markov model's (HMM) multi-level variant hierarchical hidden Markov model (HHMM) approach is used for pattern recognition and event detection for the data received for this system [4]. Using this model, a feedback and decision algorithm is also developed. This approach has been applied to simulated water pressure data from RFID motes, which were embedded in metallic water pipes.
\end{abstract}

Reprinted from Sensors. Cite as: Nasir, A.; Hussain, S.I.; Soong, B.-H.; Qaraqe, K. Energy Efficient
Cooperation in Underlay RFID Cognitive Networks for a Water Smart Home. Sensors 2014, 14,
18353-18369.

\section{Introduction}

The pressure provided by pumps and roof tanks is needed to deliver water to consumers. From this pressure information, we can determine the water flow, which is the direct measure of the water usage. The pressure change gives unique signatures for different taping points, like the kitchen sink, the washing machine, the wash basin and showers. Furthermore, the change in pressure information in a single section of the pipe shows a possible leak or seepage. In our previous work, using PipeSense [1], an RFID-based in-pipe monitoring system, we have determined the feasibility of developing a monitoring system to measure the quality of water. The shrinking water resources all around the world have made it absolutely necessary for us to conserve water; this fact has also made water quite expensive. Usually for us, there is no way to know how much water has already been consumed, nor the cost of this consumption, until the bill arrives at the end of the month. Moreover, the bill is not easy to interpret, and it is difficult to extract information about consumption during 
a particular period of time. Secondly, there are unpredictable leaks or seepage occurs in the water pipes, accumulating the costs of the utility, the details of which remain unobserved; leaks alone are the reason for considerable loss in terms of money and resources per year. Repairing these leaks also incurs a cost and leads to the wasting of time and effort. We lack a wireless automatic system that can measure water usage in real time, monitor events, such as tap opening and closing, daily consumption patterns and predict possible leaks and seepage developing in the pipe network. For this system, we need to come up with methods and algorithms to localize the leaks and seepage in smart homes.

RFIDs integrated with pressure sensors are embedded in the pipe infrastructure. They collect pressure information and send it along with their IDs to the reader/writer destination node. From the available pressure data from the sensors, the determined usage patterns, tap events and their patterns assist the real-time control of the home water system. The information from the sensors is then run by the algorithms on the cyber system to render decisions in order to support the hardware controllers responsible for managing the water distribution parameters. Several methods have been used in the setting of multi-agent systems; however, we have chosen the hierarchical hidden Markov model (HHMM), which is a famous tool for pattern recognition [4,5]. The framework described in [4] is an integrated set of two separate four-tier frameworks; here, two HMMs were discussed for determining the $\mathrm{CO}_{2}$ and $\mathrm{CO}$ levels from the vehicle's exhaust. These two HMMs were separate and are not dependent on each other, as the data is coming from two different sensors; while the HHMMs proposed in our current proposal are hierarchical in nature, due to the fact that the consumption patterns, tap and seepage event data are interdependent or, in some other way, coming from the same sensors. Normally, HMMs are used as a speech recognition tool world over. Due to the similarities of the sensor data that we received from the pipes to speech signals, we adopted the HHMM to solve the problem presented in the proposed solution. We can also make the smart home water system consumer friendly by making the data visualization easy to interpret, along the same lines as [6]. The integrated RFID motes collect and send the data to the reader/writer mote. We propose an event detection and prediction scheme based on HHMM, which is a hierarchical variant of HMM [7]. Our approach would be to detect the differences by statistically comparing the observed pattern with that predicted by a model to discover events of interest while minimizing the delays and false alarms. A preliminary version of this research appeared in [3]. The framework described in [3] is utilized for a human-centric in-pipe water distribution monitoring system (WDS) with the goal of determining the patterns and probabilities of future water demand, water quality and contamination spread using HMM; while our proposed work uses HHMM instead of HMM to determine the consumption pattern, tap events and seepage events in the context of a water smart home.

The term "smart home" has been used to introduce the notion of networking devices and equipment that distribute information and commands among the networked devices in the home via wired and wireless communication [8]. A smart home can accommodate a number of information gadgets, home appliances and other Internet-based applications, which can communicate with each other, forming a ubiquitous home network system [9]. This presents the idea of having a server-based home gateway system, which becomes the brain of the smart home, which will surely make life easier for the common household. However, there exist several other wireless devices simultaneously 
operating under the same roof. These two systems are bound to interfere with each other, resulting in higher energy consumption due to handshaking protocols. Hence, cognitive underlay networks come to the rescue in this scenario.

The available wireless spectrum has become a scarce resource, due to the rising demand for high data rate wireless services. Due to this scarcity, emergent communication systems are required to exploit the unused licensed spectrum in an opportunistic fashion [10]. It is proposed in [11] that in the case that the licensed or primary user of the spectrum is inactive, any cognitive or secondary users can use this available spectrum. When the primary user becomes active, the secondary user must switch off its communication and look for another spectrum hole. This technique is generally referred to as the interweave approach and involves spectrum sensing and detection. Generally, the overlay method simultaneously allows both the primary and secondary users to approach the spectrum; however, the interference for the primary user is subjugated by the secondary user through advanced signal processing. Another approach, called the underlay approach, allows the sharing of the spectrum by both primary and secondary users simultaneously. In this approach, the secondary user has to satisfy strict interference constraints, and its transmission power should be below a certain threshold all of the time [11]. Underlay cognitive networks use very limited transmission powers and, hence, make the system energy efficient. A subset of these RFID motes were allowed to send the data, which increases the received SNR ratios and will be useful in decision making. In our monitoring application, for better parameter monitoring, we will need to accumulate the maximum number of nodes satisfying the threshold requirements to increase the overall received SNR.

The work by Akbar et al. [12] presents the use of hidden Markov models (HMMs) to model and predict the spectrum occupancy of licensed radio bands. The proposed HMM by Akbar et al., dynamically selects licensed frequency bands for its own use and, thus, in the process, reduces the interference from and to the users, where the channel state occupancy of the licensed primary users was assumed to be Poisson distributed. Hence, here, HMMs were employed to predict the duration of the spectrum holes for primary users. On the contrary, the HMMs and HHMMs proposed in our smart home were not used by the cognitive radio to predict the spectrum holes; instead, these models were engaged during the processing of the acquired sensor data at the base station to determine a potentially hazardous event. The working and predicting process of the two HMMs discussed are fairly similar, as by inheritance, the HMMs first train themselves by using a huge amount of data in order to develop reliable prediction models. Our proposed HMM and HHMM processes are not utilized in the cognitive spectrum sensing; in our case, the spectrum sensing is done by a very simple energy detector algorithm, and HMMs were used during the processing of the sensor's data to determine an event.

In this paper, we analyzed an underlay RFID network with fixed transmission power near a primary user, which is a special case and an extension of our previous work [2]. The system model defined in [2] has a single source (non-RFID), 'L' number of relays (non-RFID) and a single destination with a primary interferer, while our proposed system is comprised of several sources (RFIDs) with a single destination and a primary interferer. Moreover, the system in our proposal is energy efficient, as compared to the one described in [2], due to the use of RFIDs. We propose a new commutative node selection criterion based on satisfying the interference constraint, while 
maintaining the maximum SNR of the node link at the destination. We derive closed form expressions for the probability density function (pdf) of the total SNR at the destination. We also derive closed form expressions for the outage probability of the system. Here, we have also discussed our approach to identify the taping and other known events in order to recognize unknown events, such as a leak or seepage, when they occur and track them, so as to have an efficient system with less false alarms. This type of system implementation can be seen as a cyber physical system (CPS), where the user can also give feedback and actuate certain mechanisms to achieve efficiency. We have opted for a cyber-physical approach for an efficient feedback control system for wireless sensor networks.

The remainder of the paper is arranged as follows. In the next section, we will give an overview of our system. Section 3 describes the system model and introduces various notations used throughout. Section 4 presents the received SNR statistics and the derivations of some important pdfs required for performance analysis at the destination. In Section 5, we give the details of our proposed cyber system model. Section 6 details the event detection analysis. Section 7 gives the system's performance analysis in terms of outage probability and a discussion on the results obtained. Section 8 describes some future directions, and finally, Section 9 concludes the paper with summarizing comments.

\section{System Overview}

In our proposed system diagram shown in Figure 1, cost effectiveness is achieved by opting for low power and low cost active RFID motes and allowing only those motes to send data that satisfy the interference threshold of the primary user. These motes can be programmed to send data after some set limit with their IDs to the reader/writer mote, and a few motes may be needed to complete the monitoring task. A simple sleep and wake-up protocol is initially utilized in the RFID motes. Once the data is received at the access mote, it first separates the data from the ID information, makes a packet and sends this packet to the water smart server through WiFi or $3 \mathrm{G}$ networks. At the server, the event detection and decision algorithms analyze the data and predict the future 50 or 100 states of the system. The RFID motes are renowned for their low power consumption; however, the reader/writer mote with a WiFi or $3 \mathrm{G}$ option will consume some energy. It will be slightly costlier to monitor the piping system than not monitoring it at all, but in the long run, it may possibly save much more by reducing maintenance and manpower costs. An Internet-based web portal application and an Android phone application running on hand held devices take the data from the server and visualize the data for the consumers. The data on the server also get archived, so that the consumers can always compare the consumption and find previous events. Due to the different nature of the events related to the timing of the occurrences, for instance the daily consumption behavior and seepage can be an hourly event, while tap opening and closing is an event that can only be detected in a few seconds' or minutes' time, this caused us to use hierarchical HMM, which will first look at the per second data, while checking for any unusual behavior leading to a tapping event; if no such event is detected for an hour, then the model shifts to the per-hour hierarchical level to detect consumption patterns and find the seepage. In the context of water consumption, it is expected that consumers' 
behavior can change when they are regularly made aware of the amount and, in particular, the cost of what they consume.

Figure 1. System diagram showing the RFID cognitive nodes, the primary interference and the smart home server as the destination.

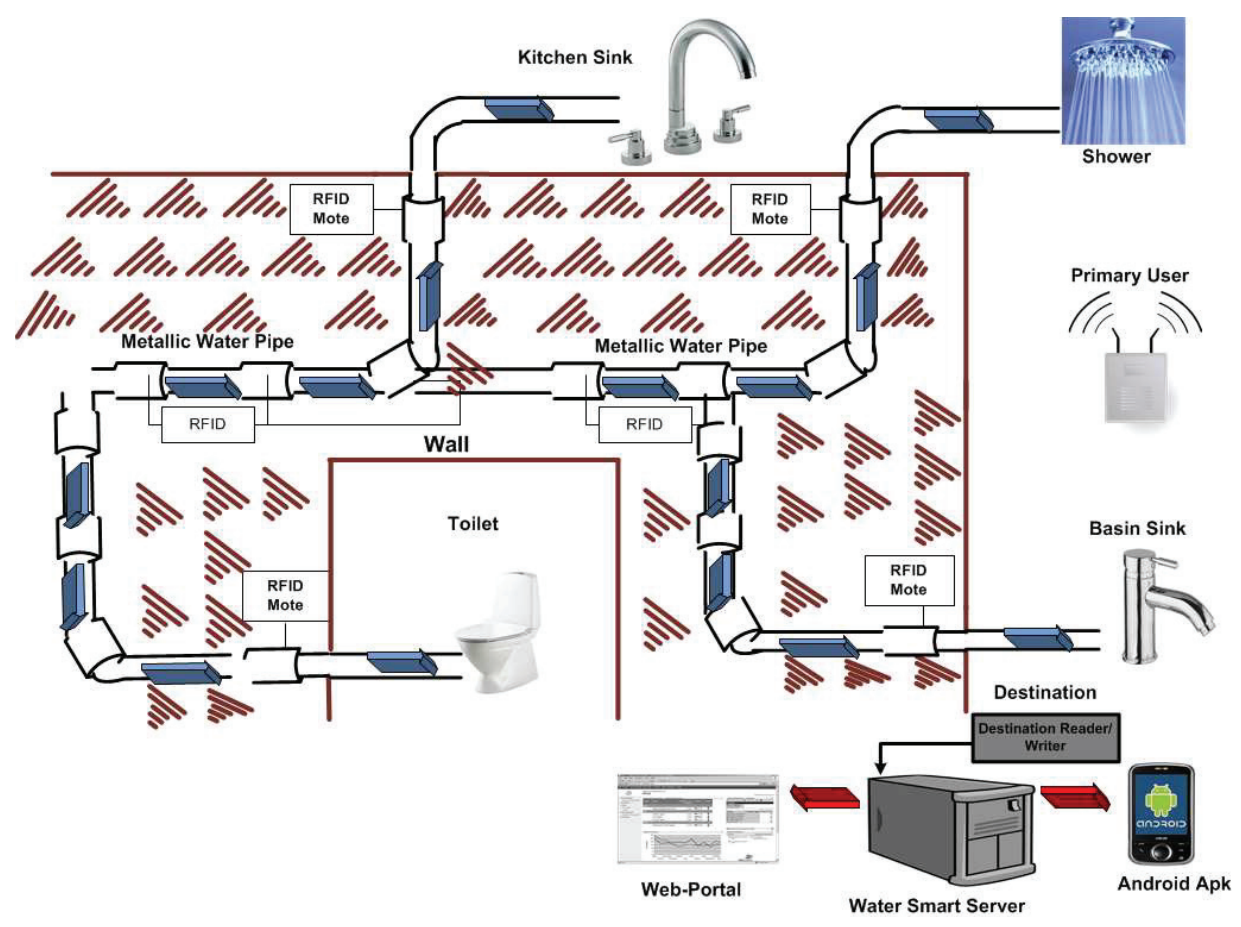

\section{System Model}

We consider an underlay cognitive RFID network operating near a primary user $P$. The cognitive network consists of $L$ secondary nodes broadcasting their data signals to the destination $D$, as shown in Figure 2. This broadcast is also received at the primary user $P$ and causes some interference. The channel coefficients from the $i-t h$ node $\left(N_{i} \rightarrow D\right)$ are $h_{i}, i=1,2,3, \ldots L$. The interference channel from the $i-t h$ node $\left(N_{i} \rightarrow P\right)$ to the primary user is $h_{i p}, i=1,2,3, \ldots L$. In addition, we assume that the channels are subjected to additive white Gaussian noise (AWGN) with zero mean and variance $N_{0}$. The available power at each node is $P$. In underlay cognitive networks, the secondary users must maintain strict interference constraint, i.e., the interference at the primary user must be below a certain threshold, say $\lambda$. Depending upon the channel gain $h_{i p}$ from the $i-t h$ node to the primary user, some nodes may not be able to satisfy the interference constraint and, hence, would refrain from sending the data to the destination. We assume that the nodes estimate their interference channel when the primary user is transmitting or acknowledging any received information. This information may also be available on a dedicated feedback channel from the primary user to the $i-t h$ node in the form of a yes or no decision. Let us say $l$ nodes satisfy the 
interference constraint out of the available $L$ nodes. We group the node indexes into different sets, such as $U$, representing the set of all node indexes and $A \subseteq U$, the set of $l$ node indexes satisfying interference constrain. We assume that all of the channel coefficients are Rayleigh distributed, and hence, their squared amplitudes are exponentially distributed.

Figure 2. System model: RFID cognitive nodes, the destination node and the primary interferer.

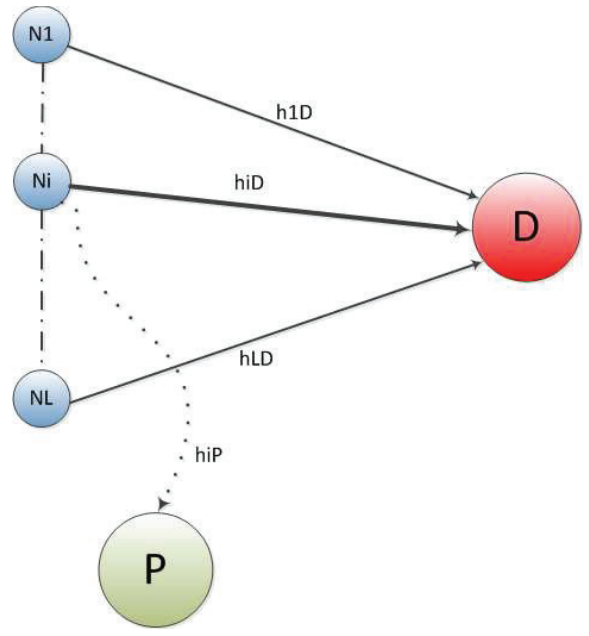

The received SNR from the $i-t h$ node transmission can be given as:

$$
\gamma_{i}=\frac{P\left|h_{i}\right|^{2}}{N_{0}}
$$

Similarly, the interference for the primary user due to the $i-t h$ node is given by:

$$
I_{i p}=P\left|h_{i p}\right|^{2} \leq \lambda
$$

\section{Received SNR Statistics}

Based on the above discussion, the SNR received at the destination can be defined as the sum of the SNRs of all of the nodes satisfying the interference constraint. Mathematically,

$$
\gamma_{T}=\sum_{i \in A} \gamma_{i} \quad \text { such that } \quad I_{i p} \leq \lambda
$$

Since $I_{i p}$ is an exponentially distributed RV, the probability of satisfying the interference threshold is:

$$
P_{\lambda}=1-e^{-\frac{\lambda}{\sigma}}
$$

where $\sigma$ are the average strengths of the interfering channels. We have assumed that the nodes are present in the form of a cluster and are roughly at the same distance from the primary user. In this 
case, the average strengths of the interfering channels can be assumed to be the same; however, their instantaneous values may be different. Therefore, $\sigma_{1 P}=\sigma_{2 P}=\ldots=\sigma$.

Similarly, $\gamma_{i}$ are also exponentially distributed with pdf $p_{\gamma_{i}}(\gamma)=\frac{1}{\gamma_{i}} e^{-\frac{\gamma}{\gamma_{i}}}$ and cumulative distribution function (cdf) $P_{\gamma_{i}}(\gamma)=1-e^{-\frac{\gamma}{\gamma_{i}}}$, where $\gamma_{i}$ is the average SNR of the $i-t h$ node branch.

It is important to note that the number of nodes satisfying the interference constraint, i.e., $l$ may vary from zero to $L$. If $l=0$, the destination would not receive any signal from the nodes. For $l=1$, only one node will be sending the data, hence no SNR aggregation is possible. In fact, the node SNR aggregation begins with $l=2$, but the events with $l<2$ should be included when evaluating the averages of various performance parameters. Hence, in summary, $l$ nodes out of a total $L$ can satisfy the interference constraint $\lambda$ with a probability $P_{\lambda}$. This dictates Bernoulli's distribution; however, in order to average over all possible values of $l$, a binomial distribution should be used, which is given below:

$$
p_{l}\left(l ; L ; P_{\lambda}\right)=\left(\begin{array}{c}
L \\
l
\end{array}\right) P_{\lambda}\left(1-P_{\lambda}\right)^{L-l}
$$

where $\left(\begin{array}{l}L \\ l\end{array}\right)=\frac{L !}{l !(L-1) !}$.

The conditional pdf of the sum of SNR among the $l$ nodes can be obtained by the following expressions.

$$
\begin{gathered}
p_{\gamma_{T}(\gamma \mid l)}=\frac{1}{\bar{\gamma}_{i}} e^{-\frac{\gamma}{\gamma_{i}}} ; l=1 \\
p_{\gamma_{T}(\gamma \mid l)}=\left[\prod_{i \in A} \frac{1}{\gamma_{i}}\right] \sum_{j \in A}\left(\frac{e^{-\frac{\gamma}{\gamma_{j}}}}{\prod_{k \in A}\left(\frac{1}{\bar{\gamma}_{k}}-\frac{1}{\bar{\gamma}_{j}}\right)}\right) ; l \geq 2
\end{gathered}
$$

The unconditional pdf of the SNR at the destination through the node aggregation can be obtained by averaging the conditional pdf over the pdf of $l$, given as:

$$
p_{\gamma_{T}(\gamma)}=\sum_{l=1}^{L}\left(\begin{array}{l}
L \\
l
\end{array}\right) P_{\lambda}^{l}\left(1-P_{\lambda}\right)^{L-l} p_{\gamma_{T}(\gamma / l)}
$$

Hence, substituting Equation (6) in Equation (8), we have,

$$
\begin{aligned}
& p_{\gamma_{T}(\gamma)}=\underbrace{P_{\lambda}^{1}\left(1-P_{\lambda}\right)^{L-1} \sum_{i=1}^{L} \frac{1}{\bar{\gamma}_{i}} e^{-\frac{\gamma}{\gamma_{i}}}}_{l=1}+ \\
& \underbrace{\sum_{l=2}^{L}\left(\begin{array}{l}
L \\
l
\end{array}\right) P_{\lambda}^{l}\left(1-P_{\lambda}\right)^{L-l}\left[\prod_{i \in A} \frac{1}{\gamma_{i}}\right] \sum_{j \in A}\left(\frac{e^{-\frac{\gamma}{\gamma_{j}}}}{\prod_{k \in A}\left(\frac{1}{\bar{\gamma}_{k}}-\frac{1}{\bar{\gamma}_{j}}\right)}\right)}_{l \geq 2}
\end{aligned}
$$

The cdf of $\gamma_{T}$ can be obtained by integrating Equation (9) from zero to $\infty$ given below: 


$$
\begin{aligned}
& P_{\gamma_{T}(\gamma)}=\underbrace{P_{\lambda}^{1}\left(1-P_{\lambda}\right)^{L-1} \sum_{i=1}^{L}\left(1-e^{-\frac{\gamma}{\gamma_{i}}}\right)}_{l=1}+ \\
& \underbrace{\sum_{l=2}^{L}\left(\begin{array}{c}
L \\
l
\end{array}\right) P_{\lambda}^{l}\left(1-P_{\lambda}\right)^{L-l}\left[\prod_{i \in A} \frac{1}{\gamma_{i}}\right] \sum_{j \in A}\left(\frac{\bar{\gamma}_{i}\left(1-e^{-\frac{\gamma}{\gamma_{j}}}\right)}{\prod_{k \in A}\left(\frac{1}{\bar{\gamma}_{k}}-\frac{1}{\bar{\gamma}_{j}}\right)}\right)}_{l \geq 2}
\end{aligned}
$$

\section{Performance Analysis}

In this section, we derived closed form expressions for the outage probability of the system using the results obtained in the previous section.

\subsection{Outage Probability}

A communication system is said to be in an outage when the received SNR is fallen below a certain threshold $\eta$. The total SNR expression in Equation (9) is for the situation when at least one node satisfies the interference constraint. However, as mentioned earlier, it is possible that none of the relays satisfy the interference constraint. The probability of this event is $\left(1-P_{\lambda}\right)^{L}$. Hence, the outage probability of the system can be evaluated as:

$$
P_{\text {out }}=P_{\gamma_{T}}(\eta)+\left(1-P_{\lambda}\right)^{L}
$$

\section{Numerical Results}

In this section, we present simulation results to verify the derived analytical expressions. First, we define the parametric setup for the simulations, and later, the results are discussed in detail.

\subsection{Simulation Setup}

All of the simulation results are generated by varying the average $\operatorname{SNR} \gamma_{i}$, where $\gamma_{i}=\alpha \_i \frac{P}{N_{0}}$. The value of $\alpha=0.1$ in the case of an interference channel. The noise is considered to be AWGN with zero mean and unit variance. The transmission power at the nodes is also assumed to be $P_{N}=1$. The maximum number of nodes in the system is $L=5$, and all of the situations are compared with equal power conditions.

\subsection{Discussion}

The outage performance of the system is plotted in Figure 3 with $L=1,2,3,4,5$, and the values of interference constraint and outage threshold are set to be 10 . The outage graph initially follows the regular water-fall curve, until it reaches a point where the nodes started to have better SNR than the outage threshold. At this point, the outage graph shows an increase of probability. As the number 
of nodes increases, the outage graph's probability values decrease steeply (shown as a solid line). On the same graph, we have shown the case of the interference constraint having a very large value (shown as a dashed line); all of the nodes satisfy the interference constraint all of the time, and hence, the system acts like a non-cognitive network. The graph shown in Figure 4, is with $L=5$ and outage threshold $=10$ with varying interference constraint $\lambda=1,5,10,20$. The figure shows that the elbow point, indicating the event when the nodes started to meet the interference constraint, slopes down with the increase in the interference constraint.

Figure 5, shows the outage probability when $L=5$, and the interference constraint is set to $\lambda=10$ and varying the values of the outage threshold $\eta=5,10,20,30,40$. It shows that the probability decreases with the increase in the outage threshold.

Figure 3. Outage probability of the system with $L=1,2,3,4,5$ for interference constraint $\lambda=10$ and outage threshold $\eta=10$.

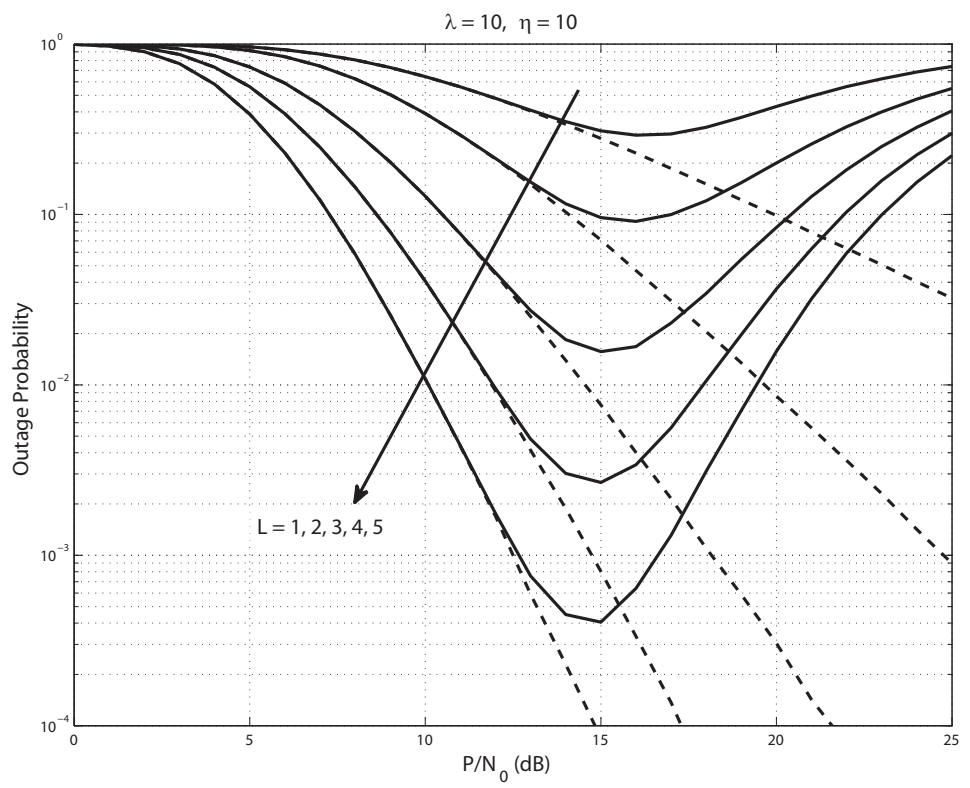


Figure 4. Outage probability of the system with $L=5$ for interference constraint $\lambda=1$, 5, 10, 20 and outage threshold $\eta=10$.

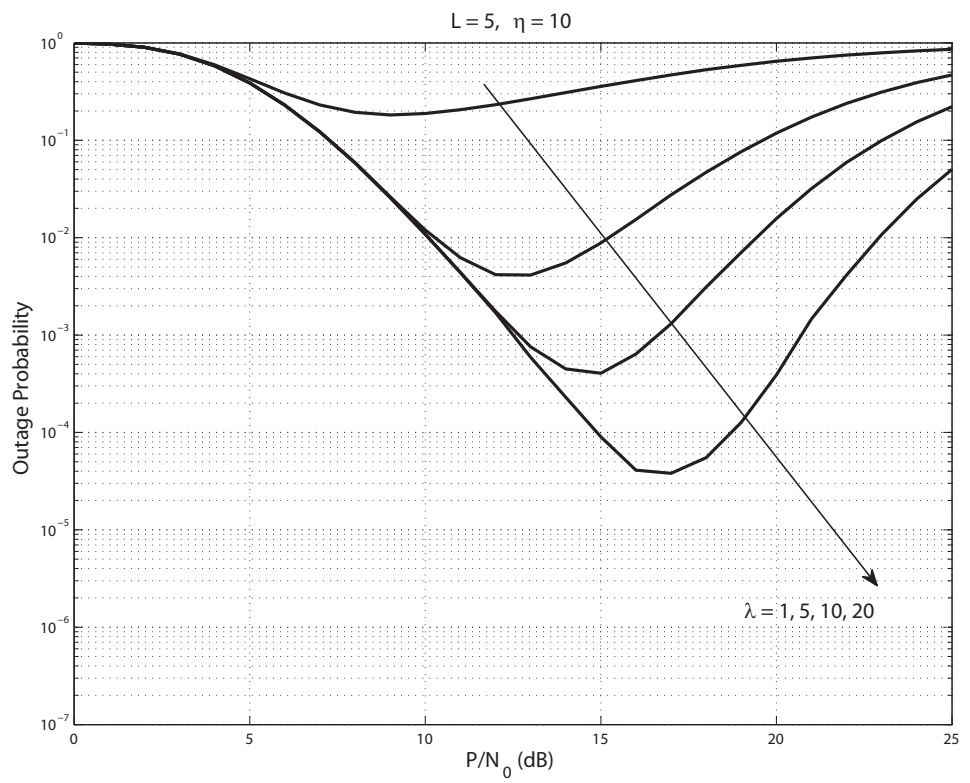

Figure 5. Outage probability of the system with $L=5$ for interference constraint $\lambda=10$ and outage threshold $\eta=5,10,20,30,40$.

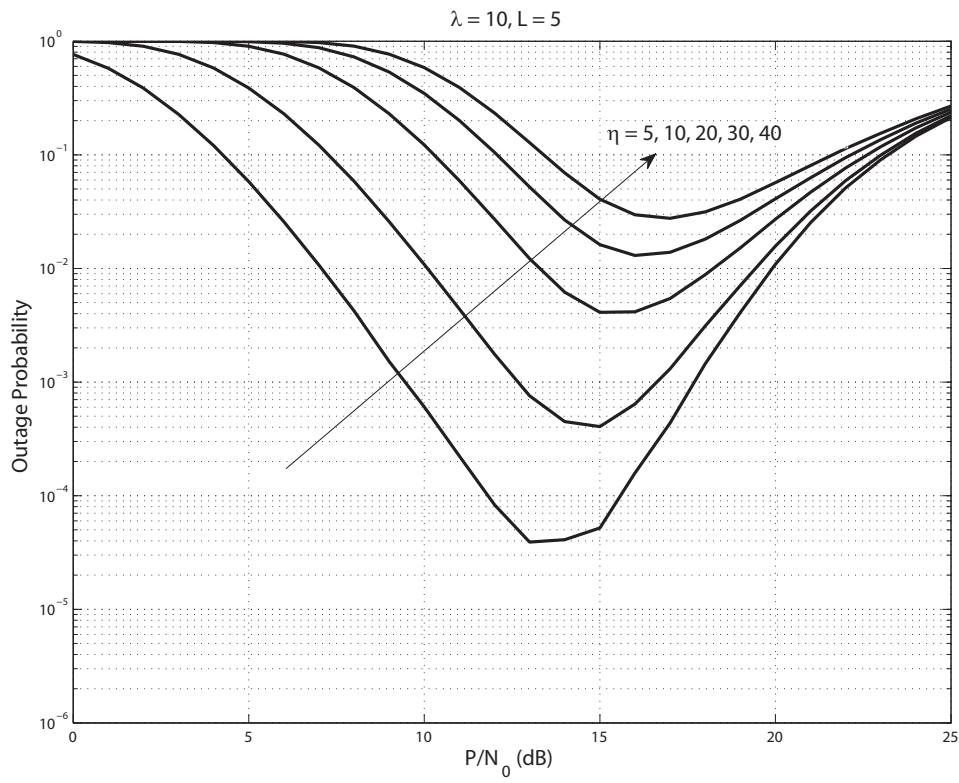




\section{Proposed Model for Cyber System}

Our smart home system diagram is shown in Figure 1. Our proposed model will allow us to detect, as well as to predict various events of interest. Every state is associated with a probability distribution over the possible output symbols. In our context, an event can be a tap event, high/low usage of water and/or seepage. The HHMM to solve and predict the tap, seepages and consumption pattern events is shown in Figure 6. An off-the-shelf 125-KHz RFID module was utilized in the experiments. Pressure sensors were deployed at the main tapping points to emulate the taping and seeping events. However, due to the requirement of a large data set in order to predict the events correctly, pseudo pressure data closely matching the actual received data was constructed to get the predictions.

Figure 6. Proposed HHMM for event detection and future event prediction. (CP, consumption pattern event; T/SE, tap and seepage event.)

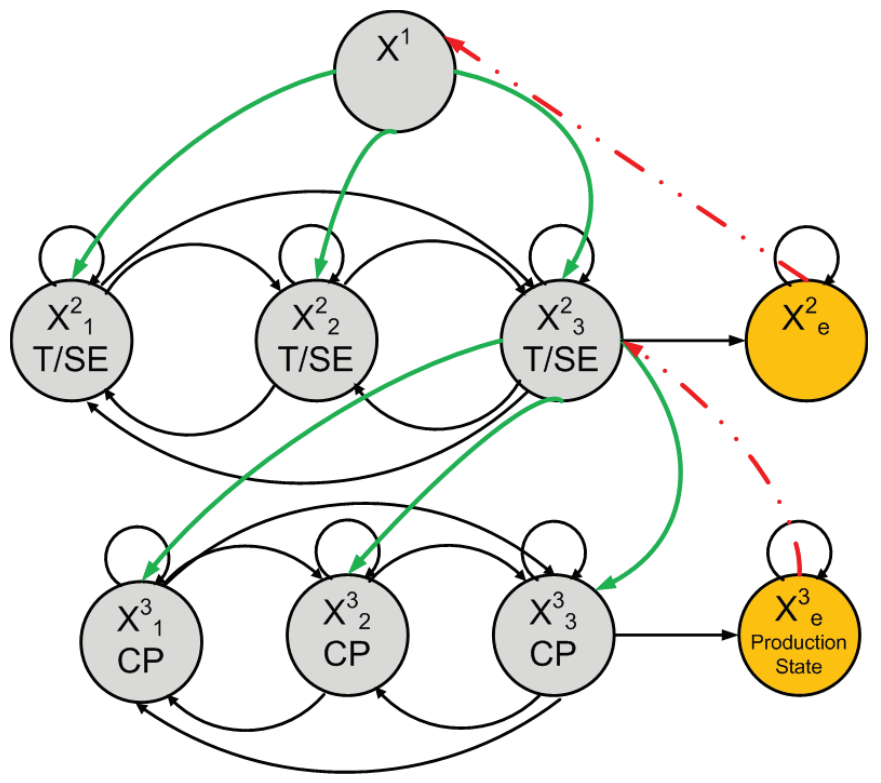

\subsection{Hierarchical Hidden Markov Models}

Hierarchical hidden Markov models [7] are simplifications of HMMs, which provide an answer to two main problems that arise in complex sequence modeling. At first, HHMMs can correlate events that happen comparatively distant from each other in an observation sequence and still maintain the ease and flexibility of a simple Markov process. Hierarchical hidden Markov models (HHMMs) are structured multi-level stochastic processes. HHMMs make each of the hidden states an autonomous probabilistic model, hence making each state an HMM, as well. Therefore, the states of an HHMM emit sequences rather than a single symbol. Figure 6 shows our HHMM to determine consumption patterns, tap events and to predict seepage events hierarchically; the third state is also an HMM to detect the usage patterns on top of seepage and tap events. An HHMM works by generating sequences 
repeatedly through activating one of the lower states of a selected state, since each HHMM is generally made up of a standard single-level HMM. Therefore, the individual states of the HMM are, in fact, the production states of the next HHMM, having a non-zero probability of going from any one state to another state. This process of recursive activations ends when we reach a special state, called the production state. In our context, the production state is the final state of the third-tier hierarchy of consumption patterns. The output symbols are only emitted through the production states; this output symbol is picked out from the set of output symbols according to a probability distribution, while the internal states are hidden states that do not give off observable symbols directly. The control then returns to the state that initiated the algorithmic activation chain. This constitutes a tree structure, where the node at the top of the structure is called the root state, while the production states makes the leaves.

\section{Event Detection and Analysis}

To determine patterns, a model has to be formulated that can be utilized to identify real instances of abnormal events from the suspicious ones. Because of this, the model should detect possible abnormal activities, expeditiously examine large sets of data and produce hypotheses with only partial and fallible information. The transition-based model shown in Figure 6 identifies the event by comparing the anticipated outputs with the data sets from different nodes in the network. It is used to detect the water consumption patterns, seepage and tap events hierarchically. We have used the expectation-maximization (EM) algorithm for HMM parameter training and performed the hypothesis testing using the maximum likelihood (ML) principles from [1,4] to identify the data samples as either normal, a possible event or a confirmed event during the recognition phase of the algorithm; as the stability is directly proportional to the amount of data collected and the number of events occurring. Our proposed system will periodically train itself, and as the data set grows, the stability increases. the initial transition matrix when there were no real data sets available will be stationary, while we next obtain the matrix of transition and emission probabilities. Here, we estimate these probabilities based on our pseudo data. The emission matrix is initialized based on the following assumptions; if usage increases, then there is a $30 \%$ chance of the need to repair and expand the water supply network. If seepage and leak events increase, then there is a $50 \%$ chance that we need to repair the system. If the water utility cost increases, then there is a $20 \%$ chance that we need to conserve water. We have logically assumed the emission matrix with a $30 \%$ chance for the water system expansion and a 50\% chance for repair. This matrix can be determined through real analysis of the home piping system during one year. In short, we can set the emission matrix values on the analyzed real data, but here, for simplicity, we assumed the matrix. Furthermore, we can map the location of the seepages and can alert households to take evasive actions. After collecting a sufficiently large data set, large enough to train our model to give meaningful predictions and decisions, we can tune the model parameters, such as the transition, emission and initial probabilities, so as to maximize the system's event detection performance. For instance, a data set of a mere few hours is sufficient for the detection of a taping event, while a consumption event will require at least the data of the whole day. Given this model, we use the forward HMM algorithm to generate 
predicted states for the three features of interest illustrated in Figure 7. The predictions were made after adjusting the transition and emission probabilities over 500 iterations.

Figure 7. Pseudo pressure values in 'bar' on the y-axis for (a) consumption pattern (CP) (per hour on the X-axis), (b) tap event (TE) (per second on the X-axis) and (c) seepage event (SE) (per second on the $\mathrm{x}$-axis).
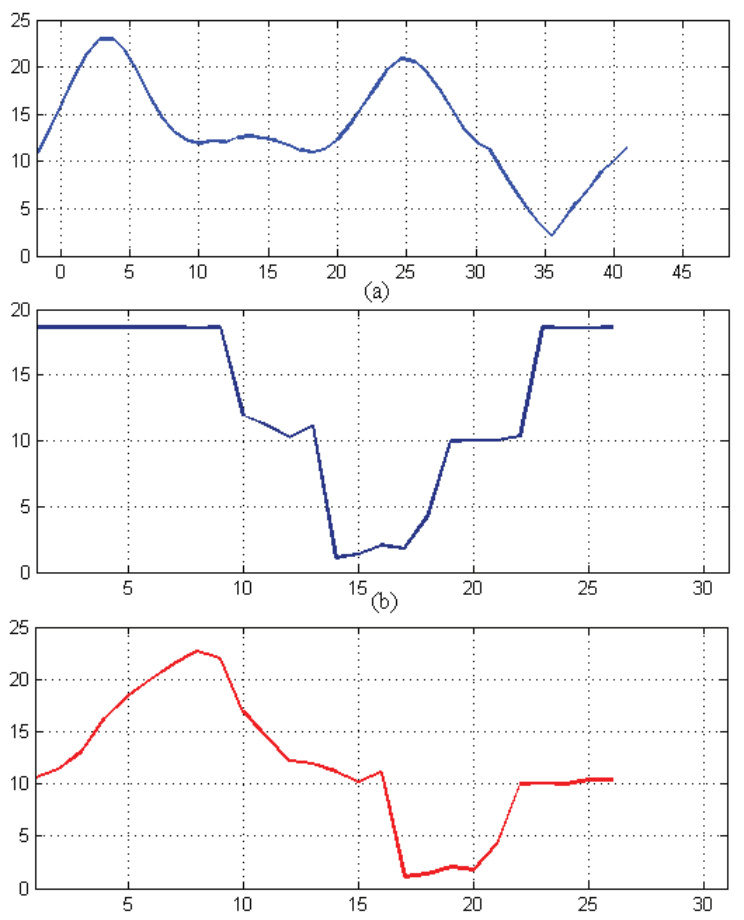

(c)

Tap events occur within the consumption pattern measurements; this makes it easier and efficient to model using a single HHMM rather than two HMMs. HHMMs train themselves after getting hourly, daily and monthly data and determining the events from this data set, so it may take as short as a couple of hours to capture the tapping events and a minimum of two days to determine the daily consumption patterns. Figure 7 shows the pseudo daily consumption pattern and tap event pressure data. These data are quantized to determine the systems' observation sequence states in Figure 8. The HHMM starts with the root state, which can, in turn, activate and pass the control to any one of the internal states at the second hierarchy, according to an initial probability matrix. The third sub-state of the second hierarchy of the consumption measurements can also pass the control to the tap event states at the third hierarchy, according to its own initial non-zero probability matrix. The third tier predicts the tapping event, generates the outcome and passes the control to the hierarchy that activated it through the production state. After that, the second hierarchy completes its generation of the predicted events and passes the control back to the root state. As the data set grows and the system comes across a number of events, our proposed HHMM trains itself using daily and 
monthly event data sets. This training and parameter determination can be done in a few minutes. Figure 9 shows the generated state prediction for the two events. HHMM generated prediction states indicate what may be the expected behavior of the system. Given the current obtained readings, the higher the prediction span, the more complex will be the decision. Therefore, we need an algorithm to detect these predictions to come up with any sort of decision. These HHMM-based predictions for 300 points of data from each node are given to a decision algorithm, which searches for 50 or more continuous State 3 predictions in the predicted sequence for each node and compares them with the other nodes in the region. If three or more nodes show similar predicted behavior, then a SMS alert is sent to the households and an alarm signal to the water smart server. The process then waits for acknowledgment, and after receiving it, the system provides visualization of the event with the location of the event. These 300 points indicate hours in the case of the consumption pattern and seconds in the case of tap and seepage events. In other words, we can make HHMM predict a sequence of any number of points (hours, seconds) regarding the consumption, tap and seepage events. The output of Figure 9 shows that the predicted events hop around in three distinct states. If the predicted pattern depicts more transactions in State 3, this means that we have more chances of having an actual event in the future, and if the predicted sequence mostly remains in the second or first states, the chances of having this event are very rare.

Figure 8. (a) Consumption patterns (CPs) (per hour on the $\mathrm{x}$-axis); (b) tap event (TE) (per second on the $\mathrm{x}$-axis); and (c) seepage event (SE) (per second on the $\mathrm{x}$-axis); quantized state sequences are on the y-axis.
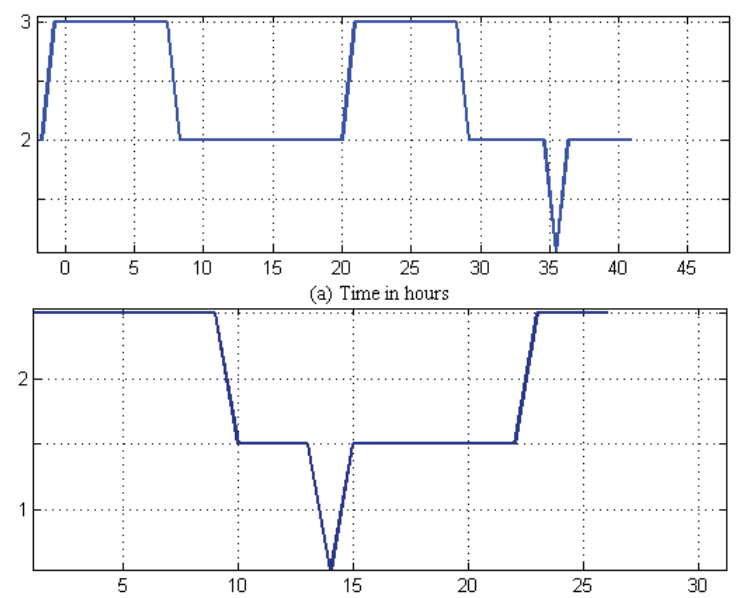

(b) Time in seconds

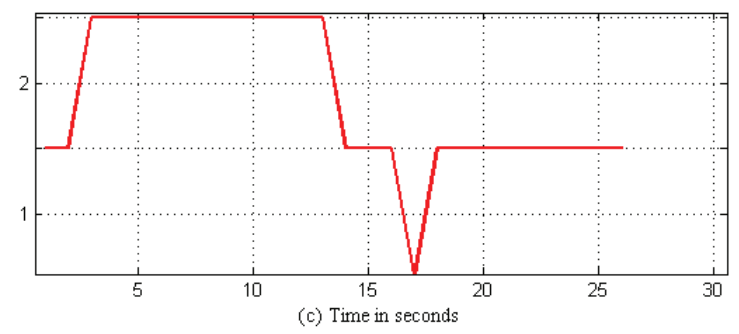


Figure 9. (a) Consumption pattern (CP) predicted states; (b) tap event (TE) predicted states and (c) seepage event (SE) predicted states against 300 future points (hours for (a) and seconds for (b) and (c)) from HHMM.
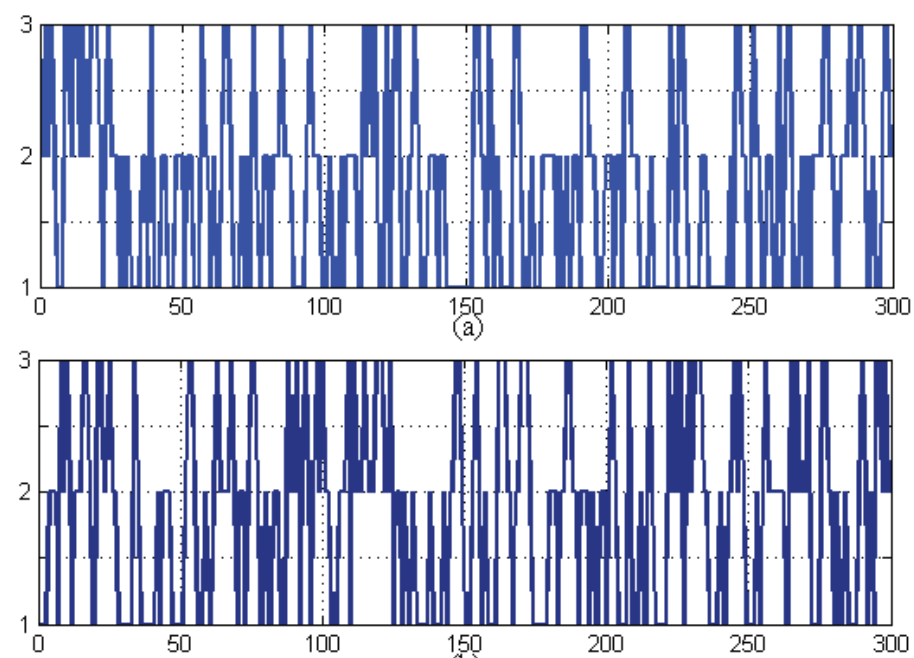

(b)

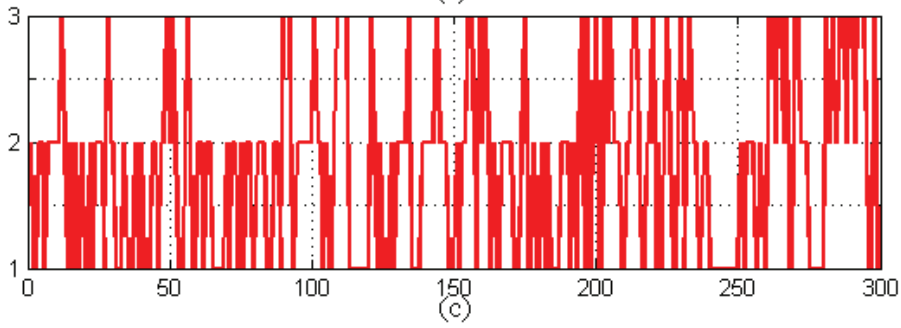

\section{Future Work}

Human-centric sensing allows us to derive the most out of water monitoring research and applications. We intend to enhance our system with a multi-interface data service for administrative functions and a map service for normal users who are interested in the overall consumptions and ways to conserve water. It can also utilize existing sensor modules and feed their data to the users' hand-held computing devices for processing and analysis. We are exploring the use of communication methods, such as WiFi, Bluetooth, etc. These provide a wide choice of data transfer speeds and flexibility in building a network. At the later stage of the research, an improved energy-efficient event-driven MAC protocol for in-pipe RFID motes can be introduced. Some of the applications where this system can also be applied are sewage monitoring, oil and gas installations, industrial gas leak detections and quality management. 


\section{Conclusions}

We proposed a best node selection scheme for a cognitive network operating near a primary user. We deduced through analysis and simulations that SNR is not the only criterion to pick up the best node in the cognitive setting. The proposed scheme works by first eliminating those nodes that do not satisfy the interference constraint. Then, among those nodes that successfully satisfy the constraint, the one giving the maximum end-to-end SNR is chosen to forward the source message to the destination. We derived the closed form pdf of the total SNR at the destination using the MGF approach and then used it to derive BER and outage probability in closed forms. Analytical formulae are verified through simulations. Some important features and tradeoffs of the proposed scheme are also discussed. Our key interest is to conserve water and control the cost by sensing various aspects of the water network in a water smart home and to share the information to users to control, preserve and improve their life styles. The end-user, which can be a household or an authority, is informed each day about the consumption and can decide how to be more efficient. This paper presents a human-centric CPS cycle for an in-pipe water monitoring system. The CPS cycle and the proposed models for consumption patterns and tap opening/closing events for the water network system have been described. We also have described the application of HHMMs for modeling the system and their use for detecting events, identifying patterns in the data, predicting events and in making decisions. We also presented modeling results and analysis based on the pseudo pressure data. In addition, directions for further research and development and its impact were presented.

\section{Acknowledgments}

The first author has moved to Qatar University Doha Qatar. This publication was made during his stay at Texas A\&M University at Qatar. The first author gratefully acknowledges the financial support from Nanyang Technological University for the Research Scholarship and the Qatar National Research Fund (a member of the Qatar Foundation).

\section{Author Contributions}

The major contributions are from Adnan Nasir and Syed Imtiaz Hussain. Adnan Nasir extended his initial findings to this journal. Boon-Hee Soong and Khalid Qaraqe are the thesis advisor and principle investigators of the projects, respectively and provided the initial motivation of the research problem.

\section{Conflicts of Interest}

The authors declare no conflict of interest. 


\section{References}

1. Nasir, A.; Soong, B.H. PipeSense A framework architecture for in-pipe water monitoring system. In Proceedings of the IEEE 9th Malaysia International Conference on Communications (MICC 2009), Kuala Lumpur, Malaysia, 15-17 December 2009; pp. 703-708.

2. Hussain, S.I.; Abdallah, M.M.; Alouini, M.-S.; Hasna, M.; Qaraqe, K.A. Performance analysis of selective cooperation in underlay cognitive networks over Rayleigh channels. In Proceedings of the IEEE 12th International Workshop on Signal Processing Advances in Wireless Communications (SPAWC 2011), San Francisco, CA, USA, 26-29 June 2011; pp. 116-120.

3. Nasir, A.; Soong, B.H.; Ramachandran, S. Framework of WSN based human centric cyber physical in-pipe water monitoring system. In Proceedings of the 11th International Conference on Control, Automation, Robotics and Vision (ICARCV 2010), Singapore, 7-10 December 2010.

4. Nasir, A.; Lim, H.B.; Soong, B.H. An integrated vehicular and urban sensing system: Framework and modeling. In Proceedings of the International Conference on Information Networking (ICOIN 2012), Bali, Indonesia, 1-3 Feburary 2012; pp. 159-164.

5. Fine, S.; Singer, Y.; Tishby, N. The Hierarchical Hidden Markov Model: Analysis and Applications. Mach. Learn. 1998, 32, 41-62.

6. Petersen, J.E.; Shunturov, V.; Janda, K.; Platt, G.; Weinberger, K. Dormitory residents reduce electricity consumption when exposed to realtime visual feedback and incentives. Int. J. Sustain. High. Educ. 2007, 8, 16-33.

7. Rabinar, L.R. A tutorial on Hidden Markov Models and selected applications in speech recognition. IEEE Proc. 1988, 77, 257-285.

8. Bierhoff, I.; van Berlo, A.; Abascal, J.; Allen, B.; Civit, A.; Fellbaum, K.; Kemppainen, E.; Bitterman, N.; Freitas, D.; Kristiansson, K. Towards an Inclusive Future; In Proceedings of COST (European Cooperation in Science and Technology): Brussels, Belgium, 2007; Volume 77, pp. 110-156.

9. Li, X.; Xu, G.; Li, L. RFID based smart home architecture for improving lives. In Proceedings of the 2nd International Conference on Anti-counterfeiting, Security and Identification (ASID 2008), Guiyang, China, 20-23 August 2008; pp. 440-443.

10. Haykin, S. Cognitive radio: Brain-empowered wireless communications. IEEE J. Sel. Areas Commun. 2005, 23, 201-220.

11. Goldsmith, A.; Jafar, S.A.; Maric, I.; Srinivasa, S. Breaking spectrum gridlock with cognitive radios: An information theoretic perspective. IEEE Proc. 2009, 97, 894-914.

12. Akbar, I.A.; Tranter, W.H. Dynamic spectrum allocation in cognitive radio using hidden Markov models: Poisson distributed case. In Proceedings of the IEEE SoutheastCon, Richmond, VA, USA, 22-25 March 2007; pp. 196-201. 


\title{
Inverse Transient Analysis for Classification of Wall Thickness Variations in Pipelines
}

\section{Jeffrey Tuck and Pedro Lee}

\begin{abstract}
Analysis of transient fluid pressure signals has been investigated as an alternative method of fault detection in pipeline systems and has shown promise in both laboratory and field trials. The advantage of the method is that it can potentially provide a fast and cost effective means of locating faults such as leaks, blockages and pipeline wall degradation within a pipeline while the system remains fully operational. The only requirement is that high speed pressure sensors are placed in contact with the fluid. Further development of the method requires detailed numerical models and enhanced understanding of transient flow within a pipeline where variations in pipeline condition and geometry occur. One such variation commonly encountered is the degradation or thinning of pipe walls, which can increase the susceptible of a pipeline to leak development. This paper aims to improve transient-based fault detection methods by investigating how changes in pipe wall thickness will affect the transient behaviour of a system; this is done through the analysis of laboratory experiments. The laboratory experiments are carried out on a stainless steel pipeline of constant outside diameter, into which a pipe section of variable wall thickness is inserted. In order to detect the location and severity of these changes in wall conditions within the laboratory system an inverse transient analysis procedure is employed which considers independent variations in wavespeed and diameter. Inverse transient analyses are carried out using a genetic algorithm optimisation routine to match the response from a one-dimensional method of characteristics transient model to the experimental time domain pressure responses. The accuracy of the detection technique is evaluated and benefits associated with various simplifying assumptions and simulation run times are investigated. It is found that for the case investigated, changes in the wavespeed and nominal diameter of the pipeline are both important to the accuracy of the inverse analysis procedure and can be used to differentiate the observed transient behaviour caused by changes in wall thickness from that caused by other known faults such as leaks. Further application of the method to real pipelines is discussed.
\end{abstract}

Reprinted from Sensors. Cite as: Tuck, J.; Lee, P. Inverse Transient Analysis for Classification of Wall Thickness Variations in Pipelines. Sensors 2013, 13, 17057-17066.

\section{Introduction}

Pipeline deterioration is a significant problem for engineers aiming to avoid costly failures or plan rehabilitation of pipeline assets. Typical forms of deterioration in pipeline systems include: internal or external corrosion of pipe walls, loss of lining and development of tubercles. These processes can lead to failure of the system through leak development, blockage formation or pipeline bursts which can lead to costly unexpected shutdowns, fluid contamination or increased running costs. Identification of pipeline deterioration has historically been carried out through external visual inspections, meaning that the identification of internal damage was more difficult. 
The development of closed circuit television (CCTV) cameras has enabled visual inspection of pipe interiors, however its range is limited and assessments can only be made based on damage that can be visually identified. Other inspection techniques such as eddy current analysis, ground penetrating radar, magnetic flux leakage and pipeline inspection gauges (PIGs) have been developed for pipeline inspection. While these methods enable the gathering of good quality data, they can be very expensive to implement and are intrusive, requiring physical entry to a pipeline system, excavation or system shutdowns [1].

To overcome the limitations of these existing methods the concept of analysing unsteady pressure responses within pipeline systems has been of interest to many research groups and is commonly referred to as transient analysis. An unsteady pressure response in a pipeline system is affected by any structural or geometric variations within that system and, as pressure waves can travel many kilometres within a pipeline, analysis of unsteady pressure responses within a system can potentially provide continuous information about the condition of that pipeline. Many methods for fault detection through transient analysis have been proposed, for which summaries can be found in Colombo et al. [2]. One such method takes transient pressure measurements from strategically placed pressure sensors in a pipeline system. Then, the transient pressure response can be used to determine the condition and physical state of a pipeline through inversely calibrating a numerical model to match the response, hence theoretically replicating the pipeline. This method is known as inverse transient analysis (ITA) and was first proposed by Pudar and Liggett [3]. For ITA to be successfully carried out a good understanding of the unsteady fluid behaviour in complex systems is required.

Transient analysis was first investigated by Stephens et al. [4] for the purposes of internal wall condition assessments of pipelines. The authors showed that changes in the condition of wall lining in a $750 \mathrm{~mm}$ mild steel cement lined (MSCL) pipeline would create reflections which can be used to characterise wall deterioration. Stephens et al. [5] followed on with this research and presented an ITA method of condition assessment which divided the pipeline into $15 \mathrm{~m}$ long sections, then inversely selected one of five predetermined levels of pipe damage for each section in an attempt to replicate the transient response of the system. The results showed reasonable correlation between the damage predicted by the ITA method and damage determined through the commercially available methods; ultrasonic pipe wall inspections and visual closed circuit television surveys. Hachem and Schleiss [6] carried out laboratory investigations that aimed to detect deterioration of pipe walls by considering simulated weak sections in a pipeline. The analysis methods used combined fast Fourier transforms and wavelet analysis techniques to locate the weak pipe sections. The weak sections were represented by using different pipe materials over short $0.5 \mathrm{~m}$ lengths. The method enabled the location of a single weak section of pipe to be determined along with a fair approximation of the wavespeed. Gong et al. [7] presented a Time Domain Reflectometry (TDR) method for the detection of a deteriorated section in a single pipeline. The method calculates the characteristic impedance of a deteriorated section by considering the magnitude of the initial reflection, from which the wavespeed and wall thickness of the section can be calculated by considering the equation for wavespeed in a fluid filled pipe (Equation (8)) which can be found in [8]. The method is shown to produce accurate results for laboratory experiments and is computationally cheap, 
however it makes a number of assumptions that may limit its application to field based analysis. These assumptions include: that corrosion of the pipe wall only occurs internally and does not affect Young's modulus of the material; that corrosion is uniform in both radial and longitudinal directions; that no corroded material remains attached to the pipe wall and that the time of the induced head perturbation is less than the time it takes for the wave front to travel two lengths of the deteriorated section. Accuracy of the method is also subject to the operator's selection of reference data points.

To improve upon the versatility of these detection methods it is necessary to reduce the number of simplifying assumptions. This paper describes an ITA method which can account for variations in the wavespeed, diameter and length of a deteriorated section independently, thus reducing the number of assumptions to be made.

\section{Modelling Theory}

This investigation uses the Method of Characteristics (MOC) to solving the governing mass and linear momentum conservation equations for one dimensional unsteady pipe flow [8]:

$$
\begin{gathered}
\frac{g A}{a^{2}} \frac{\partial H}{\partial t}+\frac{\partial Q}{\partial x}=0 \\
\frac{1}{g A} \frac{\partial Q}{\partial t}+\frac{\partial H}{\partial x}+h_{f}=0
\end{gathered}
$$

where $H$ is the head in the pipe, $Q$ is the pipe discharge, $A$ is the cross-sectional area of the pipe, $a$ is the wavespeed, $g$ is acceleration due to gravity, $x$ is the distance along the pipeline, $t$ is time and $h_{\mathrm{f}}$ is the sum of steady and unsteady frictional head losses. The derivation of these two equations assumes that both the fluid and the pipe behave in a linear elastic fashion. The equations can be solved using the MOC through confining the solution to a grid in the time and space domains by applying the following relationship:

$$
\frac{d x}{d t}= \pm a
$$

where $d x$ is the grid spacing in the along the length of the pipe and $d t$ is the time step for the numerical solution.

Solving Equations (1) and (2) subject to the condition in Equation (3) gives two simultaneous equations which can be used to solve for the head $\left(H_{P}\right)$ and flow $\left(Q_{P}\right)$ at a grid point where the head $\left(H_{A}, H_{B}\right)$ and flow $\left(Q_{A}, Q_{B}\right)$ are known values at adjacent nodes in the previous time step:

$$
\begin{aligned}
& H_{P}=H_{A}-B\left(Q_{P}-Q_{A}\right)-R Q_{P}\left|Q_{A}\right| \\
& H_{P}=H_{B}+B\left(Q_{P}-Q_{A}\right)+R Q_{P}\left|Q_{B}\right|
\end{aligned}
$$

where $B$ is the characteristic impedance of the pipeline given by:

$$
B=\frac{a}{g A}
$$

and $R$ is the pipeline resistance coefficient, which can be calculated by: 


$$
R=\frac{f d x}{2 g D A^{2}}
$$

where $D$ is the nominal diameter of the pipe section and $f$ is the Darcy-Weisbach friction factor. The additional effects of unsteady friction can be accounted for using the efficient approximation of Vardy and Brown [9] for smooth turbulent pipe flow presented in Vitkovsky et al. [10].

The MOC model described is coded in Fortran using a constant time step discretisation such that numerical dissipation and dispersion errors that arise with the use of interpolation methods are avoided. The constant time step discretisation method requires the space step to be altered between sections of pipe to account for a change in the wavespeed as specified by Equation (3). Changes in pipeline properties in the longitudinal direction such as diameter and wavespeed can be accounted for by altering the variables in Equations (6) and (7).

\section{Numerical Analysis}

This section examines the effect that changes in pipeline wall condition have on transient behaviour within a pipeline. Changes in pipe wall conditions can alter three key parameters. The first is a change in the nominal diameter $(D)$, which can increase due to internal corrosion of the pipe wall and delamination of internal protective linings, or decrease where corrosion leads to tuberculation. The second parameter is the wavespeed $(a)$ which can be affected by the delamination of protective linings, internal and external corrosion and deterioration of the pipe wall material. The delamination and corrosion lead to a reduction in effective wall thickness, while pipe wall deterioration results in a decreased Young's modulus. The third parameter is a change in the friction factor $(f)$ caused by a change in relative roughness. For the purposes of this research the relative effect of changes in friction factor are considered to be small, therefore they are not accounted for.

Research presented in Tuck et al. [11,12] shows that a change in nominal diameter $(D)$ or wavespeed $(a)$ over a section of pipe can generate positive or negative reflections in the pressure response while also changing the fundamental period of oscillation for the system. To further illustrate these effects with a focus on the subject of pipe wall condition a numerical case study is used. This example considers a reservoir, pipe and valve system as depicted in Figure 1, where a $100 \mathrm{~m}$ long mild steel cement lined (MSCL) pipeline has lost the protective cement lining from a $20 \mathrm{~m}$ section located in the middle of the pipeline. The MSCL pipe considered has a nominal diameter $(D)$ of $300 \mathrm{~mm}$, steel wall thickness $(e s)$ of $5 \mathrm{~mm}$ and cement thickness $\left(e_{C}\right)$ of $10 \mathrm{~mm}$. 
Figure 1. Schematic of reservoir, pipe and valve system for (a) an intact, fault free pipeline and (b) a pipeline with a section of reduced wall thickness.

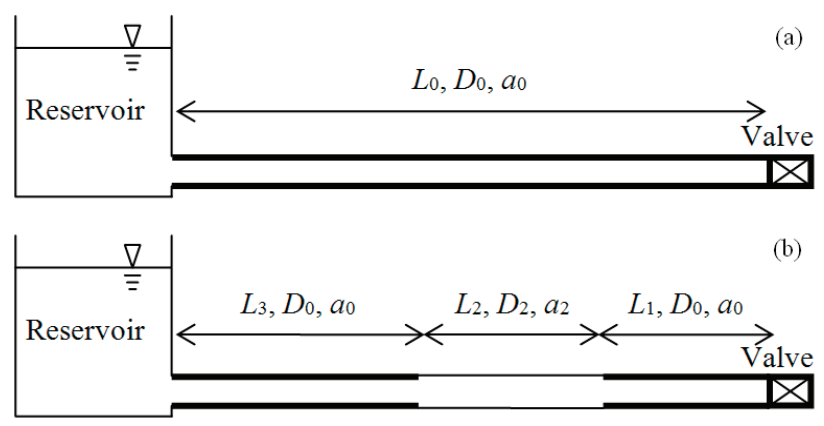

The wavespeed for a pipeline can be calculated by the wavespeed formula [8]:

$$
a=\frac{K / \rho}{1+[(K / E)(D / e)] c_{1}}
$$

where the bulk modulus of the fluid $(K)$ is taken as $2.14 \mathrm{GPa}$, the density of the fluid $(\rho)$ is $999 \mathrm{~kg} / \mathrm{m}^{3}$, $e$ is the effective wall $\mathrm{t}$

hickness, $E$ is Young's modulus of the pipe wall material and $c 1$ is a dimensionless parameter which accounts for constraint conditions on the pipeline and is taken as 1 . To account for the relative strength of the cement lining an equivalent steel thickness can be calculated by the method in Stephens et al. [5] if it is assumed to be fully bonded to the steel:

$$
e=e_{S}+e_{C}\left(E_{C} / E_{S}\right)
$$

where Young's modulus of the pipe wall steel $\left(E_{S}\right)$ is $210 \mathrm{GPa}$ and Young's modulus of the cement lining $\left(E_{C}\right)$ is $25 \mathrm{GPa}$.

Figure 2. Comparison between an intact MSCL pipeline and a pipeline which has lost lining over a length of $L_{T} *=0.2$.

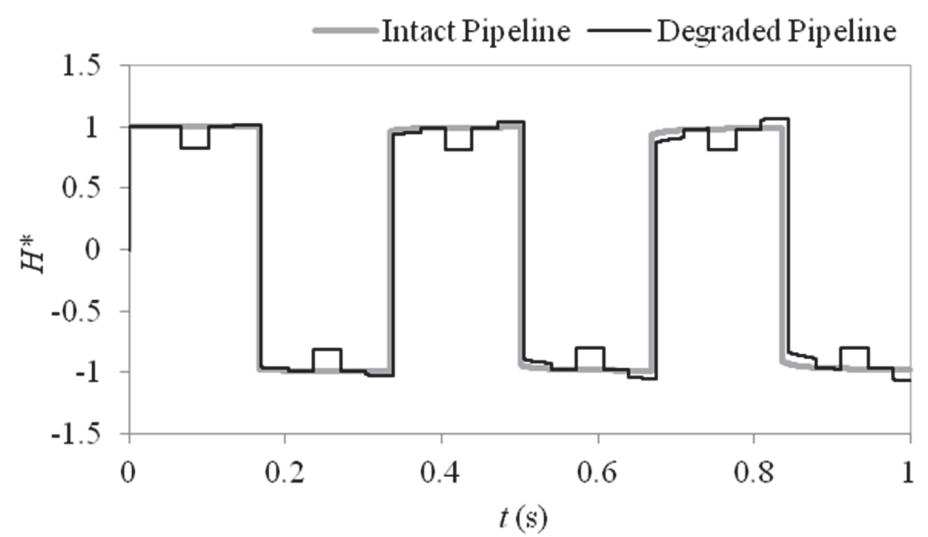


Using Equations (8) and (9) the wavespeed of the intact pipeline is calculated to be 1,197 m/s and the wavespeed over the section where the cement lining has delaminated is calculated to be $1,139 \mathrm{~m} / \mathrm{s}$. Figure 2 shows a comparison between the unsteady pressure responses from a pipeline with a section of delaminated cement lining and that of an intact pipeline as described above for this numerical case study. The unsteady pressure response is generated through instantaneous closure of the downstream online valve during fully turbulent flow conditions. The pressure head response $H(t)$ has been shown at the downstream valve and is non-dimensionalised such that $H^{*}=H / H_{J}$ where $H_{J}$ is the Joukowsky head rise. Following the initial Joukowsky head rise a reflection is observed from the downstream end of the degraded pipe section which gives a reduction in the pressure head. This reduction is caused by a decrease in characteristic impedance over the degraded section.

Following the initial drop in pressure head a reflection is observed from the upstream end of the degraded section which restores the pressure head back towards the observed values for the intact pipeline. Further secondary reflections are then observed before the pressure restoring reflection is observed from the upstream reservoir. The pressure response also shows a phase change, where the period of oscillation is increased for the system with the degraded pipeline.

\section{Experimental Analysis}

Laboratory experiments were carried out using the transient pipeline facility at the University of Canterbury to further investigate the effect that variations in pipe wall thickness have on unsteady fluid behaviour in pipelines. The experimental system consists of a $41.517 \mathrm{~m}$ long stainless steel pipeline with an external diameter of $76.2 \mathrm{~mm}$ and wall thickness of $1.5 \mathrm{~mm}$. The pipeline is bounded by a pressure tank at the upstream end to represent a constant head reservoir and a discharge valve at the downstream end which can be rapidly closed to induce unsteady behaviour. The resulting pressure response is measured at the point of generation at a sampling rate of $10 \mathrm{kHz}$ by high resolution Thermo Fisher Scientific, flush face, dynamic pressure transducers. The pressure transducers are accurate to within $\pm 1 \%$ of the magnitude of the measured signal. This error is largely linear, thus will have little effect on the comparisons between numerical and experimental responses as the magnitude of the numerical response is based upon the Joukowsky head rise. Variations in pipe wall thickness were investigated through adding a section of pipe with a wall thickness of $3.65 \mathrm{~mm}$ and outside diameter equivalent to the existing pipeline. Thicker walled pipe was used for this experiment as sections with thinner walls than the existing pipeline were not commercially available and it was not feasible to replace the whole pipeline. The length of the thick walled section $\left(L_{2}\right)$ is $10.407 \mathrm{~m}$ and it is located at a distance $\left(L_{1}\right)$ of $16.550 \mathrm{~m}$ from the downstream valve. The wavespeed of the standard pipeline $\left(a_{1,3}\right)$ is experimentally determined as $1,180 \mathrm{~m} / \mathrm{s}$ and the wave speed of the thick walled section $\left(a_{2}\right)$ is $1,315 \mathrm{~m} / \mathrm{s}$.

Figure 3 shows a comparison between the experimental pressure response and the MOC model over the first 3.5 periods of oscillation. The time step for the numerical model is taken as $0.0001 \mathrm{~s}$ to match the resolution of the experimental data which is sampled at a rate of $10 \mathrm{kHz}$. The comparison shows that the MOC model can provide a reasonable prediction of the transient behaviour and captures the complex reflection patterns induced by the thick walled pipe section. 
For this case the initial reflection observed from the thick walled section is positive as the nominal diameter is decreased and the wavespeed is increased which increases the impedance of the section, giving the opposite effect to that observed in the numerical case study. The differences observed between the numerical and experimental results can be attributed to differences in physical behaviour and in the experimental system which are not accounted for by the one dimensional model. These differences include fluid structure interaction, non-uniformities in the pipe materials due to manufacturing tolerances and additional damping from non-pipe elements such as the air bleed valves located on the experimental pipeline.

Figure 3. Comparison between numerical and experimental pressure responses for a pipeline with a thick walled section.

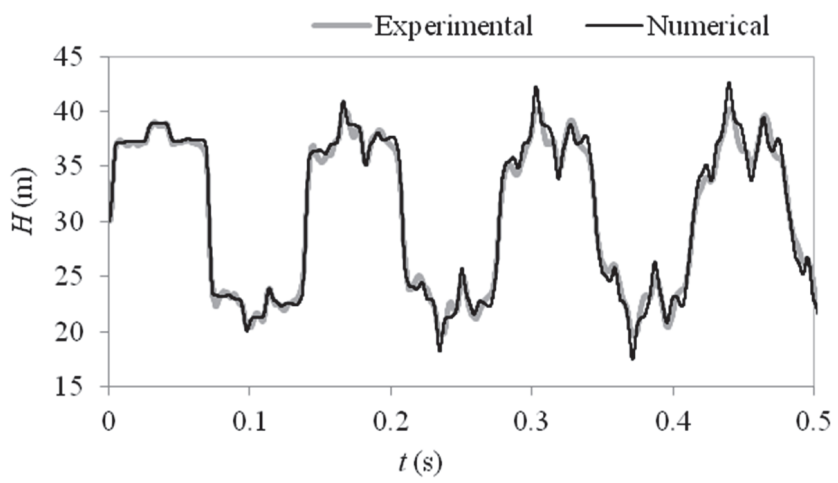

\section{Inverse Transient Analysis}

It has been shown in Figure 3 that a MOC model can produce an accurate representation of transient flow behaviour where variations in pipe wall thickness occur and the measured parameters are defined. This section considers the problem where the variables which define the "faulty" pipe section are unknown. To determine the unknown parameters the method of inverse transient analysis (ITA) can be used. This method aims to determine the values of the unknown parameters which achieve the best fit between predicted and measured pressure responses using the least squares analysis:

$$
s=\sum_{i=1}^{N}\left(H_{i}^{m}-H_{i}^{p}\right)^{2}
$$

where $s$ is the residual error, $\mathrm{N}$ is the number of data points, $H_{l}^{m}$ is the measured pressure response and $H_{t}^{p}$ is the predicted pressure response. Equation (10) can be minimised using a genetic algorithm (GA) optimisation routine, where the GA used for this research is MATLAB's inbuilt "ga" function. In this paper the inverse analysis is carried out considering only data from a single measurement location, however it has been shown that further measurement locations can improve the results of the inverse analysis [13].

For this inverse problem the variables that are said to define the faulty section are its wavespeed $\left(a_{2}\right)$, diameter $\left(D_{2}\right)$, distance from the downstream valve to the fault $\left(L_{1}\right)$ and length of the faulty 
section $\left(L_{2}\right)$. The variables are assigned the following bounds; $800 \mathrm{~m} / \mathrm{s}<a_{2}<1,440 \mathrm{~m} / \mathrm{s}$, $0 \mathrm{~m}<D_{2}<0.0762 \mathrm{~m}, 0 \mathrm{~m}<L_{1}<L_{0}$ and $0 \mathrm{~m}<L_{2}<L_{0}$ which are determined by allowing a lenient range of feasible values. To ensure that all solutions fall within the known pipeline geometry the following condition must also be met: $L_{1}+L_{2} \leq L_{0}$. Through selection of these variables and bounds the following assumptions are made; at most there is a single section of faulty pipeline, and the relative roughness does not increase significantly over the faulty section.

To improve the potential accuracy of the inverse analysis problem it is first necessary to determine an appropriate closure profile for the valve such that the head perturbation for the numerical response is similar to that of the experimental data. This step enables significantly more accurate results to be taken from the analysis and theoretically overcomes the problem discussed in Gong et al. [7], where discrepancies in the magnitude of a reflection can occur if the operation time of the transient generation device is greater than that required for a pressure wave to travel two lengths of a pipe section which causes an overlapping of the reflections. Where the profile of the generated head perturbation is appropriately matched the overlap of reflections from the front and rear of one or multiple sections can be accounted for in the simulations.

Table 1. Inverse transient analysis results.

\begin{tabular}{cccccccccc}
\hline & $\boldsymbol{a}_{\mathbf{2}}(\mathbf{m} / \mathbf{s})$ & $\mathbf{\%}$ error & $\boldsymbol{D}_{\mathbf{2}}(\mathbf{m})$ & $\mathbf{\%}$ error & $\boldsymbol{L}_{\mathbf{1}}(\mathbf{m})$ & \% error & $\boldsymbol{L}_{\mathbf{2}}(\mathbf{m})$ & \% error \\
\hline Measured Values & Case & 1315 & - & 0.0688 & - & 16.550 & - & 10.407 & - \\
$\boldsymbol{t}<\mathbf{2 L} / \boldsymbol{a}$ & 1 & 1054 & $19.8 \%$ & 0.0623 & $9.4 \%$ & 19.422 & $17.4 \%$ & 7.945 & $23.7 \%$ \\
$\boldsymbol{t}=\mathbf{4} \boldsymbol{L} / \boldsymbol{a}$ & 2 & 1336 & $1.6 \%$ & 0.0706 & $2.6 \%$ & 15.814 & $4.4 \%$ & 12.211 & $17.3 \%$ \\
$\boldsymbol{t}=\mathbf{8} \boldsymbol{L} / \boldsymbol{a}$ & 3 & 1360 & $3.4 \%$ & 0.0717 & $4.2 \%$ & 15.826 & $4.4 \%$ & 12.568 & $20.8 \%$ \\
$\boldsymbol{t}=\mathbf{1 2} \boldsymbol{L} / \boldsymbol{a}$ & 4 & 1339 & $1.8 \%$ & 0.0704 & $2.3 \%$ & 16.777 & $1.4 \%$ & 10.457 & $0.5 \%$ \\
$\boldsymbol{t}=\mathbf{1 6} \boldsymbol{L} / \boldsymbol{a}$ & 5 & 1326 & $0.8 \%$ & 0.0683 & $0.7 \%$ & 16.426 & $0.7 \%$ & 10.442 & $0.3 \%$ \\
$\boldsymbol{t}=\mathbf{1 6} \boldsymbol{L} / \boldsymbol{a}$, fixed $\boldsymbol{D}_{\mathbf{2}}=\boldsymbol{D}_{\mathbf{0}}$ & 6 & 1320 & $0.4 \%$ & 0.0732 & - & 17.543 & $6.0 \%$ & 10.215 & $1.8 \%$ \\
$\boldsymbol{t}=\mathbf{1 6} \boldsymbol{L} / \boldsymbol{a}$, fixed $\boldsymbol{a}_{\mathbf{2}}=\boldsymbol{a}_{\mathbf{0}}$ & 7 & 1180 & - & 0.0590 & $14.2 \%$ & 18.997 & $14.8 \%$ & 12.325 & $18.4 \%$ \\
\hline
\end{tabular}

Table 1 shows the results for seven ITAs, the first five of which investigate the effect that simulation time has on the accuracy of the results. In the first case the simulation time is taken as just less than the time for the pressure restoring wave to return to the downstream valve, here the accuracy of the predicted values are poor, where the error is calculated as (|Measured ValuePredicted Value $\mid$ /Measured Value) $\times 100 \%$. The accuracy is poor as no reflection from the end of the system is observed for the wavespeed to be scaled against, so the fundamental period of the system is not matched. From the second case, which is simulated over a whole transient cycle, to the fifth case, which is simulated over four transient cycles, there is an observable trend towards increasing accuracy. This trend has also been noted for leak detection application by Vitkovsky et al. [14]. Case 5 shows that a solution to the inverse problem can be determined to a high level of accuracy with relative errors between $0.3 \%$ and $0.8 \%$ for each parameter. Two factors contribute towards the improved accuracy where the inverse analysis is simulated over a longer period of time. The first is that a larger number of transient cycles will lead to a greater importance being placed upon matching the fundamental period of oscillation for the system which is affected by extended variations in pipelines [11]. The second is that the extended variations create the same change in each period of the signal and is progressively reinforced with each cycle. It should also 
be noted that confining the numerical solution domain to a grid in space and time limits the theoretical accuracy of the ITA to $d x / 2$, where $d x$ is the space step used in the numerical model as determined by Equation (3). For the fixed time step discretisation method adopted in this analysis and a specified time step of $0.0001 \mathrm{~s}$, the resolution of the MOC grid could account for an error of up to $0.36 \%$ for values of $L_{1}$ and $0.63 \%$ for values of $L_{2}$.

In an attempt to reduce the size of solution domain, cases 6 and 7 involved runs where changes in either the diameter or the wavespeed were excluded from the inverse calibration. An approximation such as this could prove useful where multiple faulty pipe sections are considered and can reduce the number of variables in the solution domain. This approximation is valid where the relative effect of one variable is much less than the others. The relative effects of diameter and wavespeed can first be considered by looking at the magnitude of the initial reflection from a fault. Scale analysis of Equation (6), which represents the pipeline impedance, indicates that for a general case the effect of a change in diameter is more important than that of a relative change in the wavespeed because the pipe area changes proportionally to the square of the diameter. For this specific set of experiments the percentage change in wavespeed is $11.44 \%$ while the change in area is similar at $11.66 \%$. However the results presented in cases 6 and 7 indicate that for an ITA carried out over four periods $(16 L / a)$ the wavespeed variable becomes significantly more important in achieving accurate detection. This can be explained by considering the phase change exhibited in the system response. The phase shift in the system response is shown to be most affected by the relative change in wavespeed by Tuck et al. [11] for the given range. Cases 6 and 7 show that the solution domain can be simplified by excluding variations in the diameter or wavespeed from the ITA, though considering both variables separately significantly improves results as shown by comparison with case 5 .

\section{Conclusions}

This paper has demonstrated how transient behaviour in a pipeline is altered by the presence of a degraded section of pipe which can be a precursor to pipeline failures. It has been shown that the degraded section can produce a reduction in wavespeed and changes in nominal diameter. These variations will alter the transient response of a pipeline, enabling transient analysis to be used to detect and classify degraded sections of a pipe. An inverse transient analysis method of fault detection has been implemented and shown to successfully determine the properties and location of a damaged pipe section. It has been demonstrated that the method can independently resolve changes in wavespeed and diameter over a wide solution space. This enables the method to be applied where prior information about a pipeline condition is minimal which is advantageous for field application of transient based condition assessment methods. The presented method has been evaluated using laboratory data which exhibit strong periodic behaviour. It is found that this periodic behaviour enables improvements in fault detection and classification accuracy. Where this periodic nature is not so strongly present, such as in large water distribution networks, improvements in accuracy could be achieved through a greater number of measurement points instead of increasing the duration of signal as considered here. Improvements in accuracy are also potentially achieved through increasing the sampling frequency and decreasing the modelled time step of the system response. For the experimental arrangement investigated it is demonstrated that the solution 
domain can be simplified through fixing the diameter variable in the ITA and varying only the wavespeed, length and location, however this reduces the accuracy of the fault detection method.

\section{Acknowledgments}

This research has been funded by the NZ Royal Society Marsden Grant UOC M1153 and the Brian Mason Trust E6022.

\section{Conflict of Interest}

The authors declare no conflict of interest.

\section{References}

1. Rizzo, P. Water and wastewater non-destructive evaluation and health monitoring: A review. Adv. Civil Eng. 2010, doi:10.1155/2010/818597.

2. Colombo, A.F.; Lee, P.J.; Karney, B.W. A selective literature review of transient-based leak detection methods. J. Hydro Environ. Res. 2009, 2, 212-227.

3. Pudar, R.S.; Liggett, J.A. Leaks in pipe networks. J. Hydraul. Eng. 1992, 118, 1031-1046.

4. Stephens, M.L.; Simpson, A.R.; Lambert, M.F. Internal Wall Condition Assessment for Water Pipelines Using Inverse Transient Analysis. In Proceedings of the 10th Annual Symposium on Water Distribution Systems Analysis, Reston, VA, USA, 17-20 August 2008.

5. Stephens, M.L.; Lambert, M.F.; Simpson, A.R. Determining the internal wall condition of a water pipeline in the field using an inverse transient. J. Hydraul. Eng. 2013, 139, 310-324.

6. Hachem, F.E.; Schleiss, A.J. Detection of local wall stiffness drop in steel-lines pressure tunnels and shafts of hydroelectric power plants using steep pressure wave excitation and wavelet decomposition. J. Hydraul. Eng. 2012, 138, 35-45.

7. Gong, J.; Lambert, M.F.; Simpson, A.R.; Zecchin, A.C.; Kim, Y.; Tijsseling, A.S. Detection of distributed deterioration in single pipes using transient reflections. J. Pipeline Syst. Eng. Pract. 2013, 4, 32-40.

8. Wylie, E.B.; Streeter, V.L. Fluid Transients in Systems; Prentice Hall: Upper Saddle River, NJ, USA, 1993.

9. Vardy, A.E.; Brown, J.M.B. Transient turbulent friction in smooth pipe flows. J. Sound Vib. 2003, 259, 1011-1036.

10. Vítkovský, J.P.; Stephens, M.L.; Bergant, A.; Lambert, M.F.; Simpson, A.R. Efficient and Accurate Calculation of Zielke and Vardy-Brown Unsteady Friction in Pipe Transients. In Proceedings of the 9th International Conference on Pressure Surges, Chester, UK, 24-26 March 2004; pp. 405-419.

11. Tuck, J.; Lee, P.; Davidson, M.; Ghidaoui, M.S. Analysis of transient signals in simple pipeline systems with an extended blockage. J. Hydraul. Res. 2013, in print.

12. Tuck, J.; Lee, P.; Kashima, A.; Davidson, M.; Ghidaoui, M.S. Transient Analysis of Extended Blockages in Pipeline Systems. In Proceedings of the 11th International Conferences on Pressure Surges, Lisbon, Portugal, 24-26 October 2012; pp. 101-112. 
13. Vítkovský, J.P.; Ligget, J.A.; Simpson, A.R.; Lambert, M.F. Optimal measurement site locations for inverse transient analysis in pipe networks. J. Water Res. Plan. Manag. 2003, 129, 480-492.

14. Vítkovský, J.P.; Simpson, A.R.; Lambert, M.F. Leak detection and calibration using transients and genetic algorithms. J. Water Res. Plan. Manag. 2000, 126, 262-265. 


\title{
On the Acoustic Filtering of the Pipe and Sensor in a Buried Plastic Water Pipe and its Effect on Leak Detection: An Experimental Investigation
}

\author{
Fabrício Almeida, Michael Brennan, Phillip Joseph, Stuart Whitfield, Simon Dray \\ and Amarildo Paschoalini
}

\begin{abstract}
Acoustic techniques have been used for many years to find and locate leaks in buried water distribution systems. Hydrophones and accelerometers are typically used as sensors. Although geophones could be used as well, they are not generally used for leak detection. A simple acoustic model of the pipe and the sensors has been proposed previously by some of the authors of this paper, and their model was used to explain some of the features observed in measurements. However, simultaneous measurements of a leak using all three sensor-types in controlled conditions for plastic pipes has not been reported to-date and hence they have not yet been compared directly. This paper fills that gap in knowledge. A set of measurements was made on a bespoke buried plastic water distribution pipe test rig to validate the previously reported analytical model. There is qualitative agreement between the experimental results and the model predictions in terms of the differing filtering properties of the pipe-sensor systems. A quality measure for the data is also presented, which is the ratio of the bandwidth over which the analysis is carried out divided by the centre frequency of this bandwidth. Based on this metric, the accelerometer was found to be the best sensor to use for the test rig described in this paper. However, for a system in which the distance between the sensors is large or the attenuation factor of the system is high, then it would be advantageous to use hydrophones, even though they are invasive sensors.
\end{abstract}

Reprinted from Sensors. Cite as: Almeida, F.; Brennan, M.; Joseph, P.; Whitfield, S.; Dray, S.; Paschoalini, A. On the Acoustic Filtering of the Pipe and Sensor in a Buried Plastic Water Pipe and its Effect on Leak Detection: An Experimental Investigation. Sensors 2014, 14, 5595-5610.

\section{Introduction}

Water distributions systems are susceptible to leakage, which results in a substantial wastage of water. The social and environmental effects due to leakage are also a matter of concern. For example, up to 4 million holes are dug in the UK each year in order to install or repair buried service pipes and cables. Recently, a survey on the costs of this installation/repair work estimated that street works cost about $£ 7$ bn in losses for the UK government income annually; $£ 5.5$ bn are due to social costs and $£ 1.5$ bn is due to damage [1].

Acoustic techniques have been used for many years in the water industry to detect leaks [2], and more recently they have been applied to locate underground pipes [3] and blockages (sediment depositions) in pipe networks [4]. Correlation techniques have been in common use for water leak detection over the last 30 years [5]. In general, these techniques work well in metal pipes, but their effectiveness in plastic pipes is limited [6]. Thus, the specific problem of detecting leaks in plastic pipes using acoustics has recently been receiving increasing attention by the research community. 
There are two fundamental issues that affect leak detection in plastic pipes: the first is that there is considerably more uncertainty in the noise propagation speed for plastic pipes (which needs to be known a priori for acoustic methods to be effective); and the second, which is more important, is that leak noise does not propagate as far in plastic pipes as it does in metal pipes [7]. Hunaidi and Chu [8] have described the frequency content present in leak signals measured on a bespoke buried plastic pipe rig located in Canada. Gao et al. [9] have also used the data collected from this rig to gain physical insight into the problems by comparing experimental results with predictions from simple models of the correlation function in plastic pipes due to leaks.

Although there is a body of work in the literature on leak detection using acoustic methods in plastic water distribution pipes, for example [5-14], apart from [11], there is no work in which there is a direct comparison between the effectiveness of correlation for leak detection using measurements of acoustic pressure, velocity or acceleration. Reference [11] describes a theoretical study on the different types of sensors and how they combine with the pipe to act as a filter of the leak noise. The aim of this paper is to validate these findings by carrying out an experimental study in a bespoke test rig in which simultaneous measurements using hydrophones (acoustic pressure), geophones (velocity) and accelerometers (acceleration) were made. Moreover, a quality measure for the data is proposed and tested experimentally as a metric of the prominence of the peak in the cross-correlation function related to the leak noise. Two sets of data are presented, one for a strong leak where there was good signal to noise ratio, and one for a weak leak where this was not the case.

The paper is organised as follows: in Section 2 an overview of the way in which a leak is located using acoustic techniques is given. In Section 3, the dynamic effects of the pipe and the sensors on the measured leak noise are described. Section 4 is devoted to the experimental work and the processing of the data, while Section 5 discusses the results. Conclusions from this work are summarised in Section 6.

\section{Overview of Leak Detection using Acoustic/Vibration Signals}

Figure 1 depicts a situation typically encountered in leak detection, in which a leak occurs at an unknown position in a buried water pipe. 
Figure 1. Schematic of buried water pipe in which sensors are positioned at access points either side of a leak.

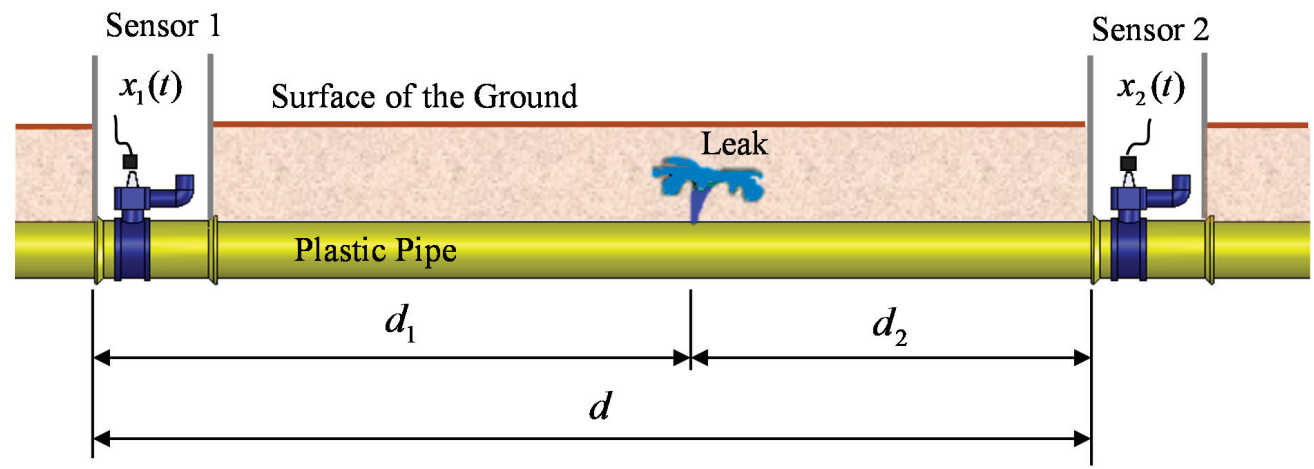

Position 1

Position 2

The leak generates broadband noise, which propagates along the pipe, both in the fluid and along the pipe-wall either side of the leak, to sensors that are located at convenient access points. These are often fire hydrants. In plastic water pipes the pipe-wall and water are strongly coupled in an acoustical sense [6,7]. This means that measurements of leak noise can, in principle, be made on the pipe or associated fittings (velocity and acceleration using geophones or accelerometers), or in the water directly (acoustic pressure using hydrophones). The difference in the arrival times of the noise at the sensors (time delay) is used to determine the position of the leak and the distance of the leak from the right-hand sensor can then be determined from [9]:

$$
d_{2}=\frac{d-c T_{0}}{2}
$$

where $c$ is the speed of propagation of the leak noise, $d$ is the total distance between the sensors, and $T_{0}=\left(d_{1}-d_{2}\right) / c$ is the time delay estimate. In many cases the wavespeed is estimated from tables, but it can be directly measured in-situ [12]. Note that it is often extremely difficult to obtain an accurate estimate of the propagation of leak noise from measurements, as the estimate is highly dependent upon the signal to noise ratio. This has a profound influence on the bandwidth over which there is useful data and this has a consequent effect on the estimate of $c$ [12].

Setting the means of the two measured signals $x_{1}(t)$ and $x_{2}(t)$ to zero, the cross-correlation function is given by [15]:

$$
R_{x_{1} x_{2}}(\tau)=\mathrm{E}\left[x_{1}(t) x_{2}(t+\tau)\right]
$$

where $\tau$ is time delay and $\mathrm{E}[\mathrm{]}$ is the expectation operator. The value of $\tau$ that corresponds to the peak in in the cross-correlation function provides an estimate of the time delay $T_{0}$. It is preferable to express the cross-correlation function in a normalized form, which has a scale of -1 to +1 . This is called the cross-correlation coefficient and is given by:

$$
\rho(\tau)=\frac{R_{x_{1} x_{2}}(\tau)}{\sqrt{R_{x_{1} x_{1}}(0) R_{x_{2} x_{2}}(0)}}
$$


where $R_{x_{1} x_{1}}(0)$ and $R_{x_{2} x_{2}}(0)$ are the autocorrelation functions at Positions 1 and 2 respectively when $\tau=0$. The cross-correlation function $R_{x_{1} x_{2}}(\tau)$, is related to the Fourier Transform of the cross-spectral density (CSD) function $S_{x_{1} x_{2}}(\omega)$ by [16]:

$$
R_{x_{1} x_{2}}(\tau)=\frac{1}{2 \pi} \int_{-\infty}^{+\infty} S_{x_{1} x_{2}}(\omega) e^{\mathrm{i} \omega \tau} d \omega
$$

where $\mathrm{i}=\sqrt{-1}$. Note that $S_{x_{1} x_{2}}(\omega)$ can be written as $\left|S_{x_{1} x_{2}}(\omega)\right| e^{\mathrm{i} \phi(\omega)}$ in which $\left|S_{x_{1} x_{2}}(\omega)\right|$ is the modulus and $\phi(\omega)$ is the phase between the two signals at frequency $\omega$. It was shown in [13] that the time delay can be determined approximately from the phase spectrum within a bandwidth of $m$ discrete frequencies. It is given by:

$$
T_{0} \approx-\frac{\sum_{j=1}^{m}\left|S_{x_{1} x_{2}}\left(\omega_{j}\right)\right| \phi\left(\omega_{j}\right) \omega_{j}}{\sum_{j=1}^{m}\left|S_{x_{1} x_{2}}\left(\omega_{j}\right)\right| \omega_{j}^{2}}
$$

Note that Equation (5) is exact only if the phase is linear (pure delay) without any significant distortion, e.g., distortions due to the dynamics of the system, and if the phase passes through the origin (i.e., $\phi=0$ at $\omega=0$ ). Otherwise it provides a least-square best-fit of the time delay estimate. Moreover, the correct choice of the frequency bandwidth over which the calculation is performed is essential for the accuracy of this estimate [12,13]. Given these conditions it is possible to determine the time delay from the gradient of a straight line fit to the phase spectrum weighted by the modulus of the cross-spectrum at each frequency. This demonstrates the importance of the modulus of the cross-spectrum, as well as the phase in the calculation of the time delay.

\section{Filtering Effect of the Pipe and Sensors}

In this section, the simple model of the pipe-sensor system proposed in [11], is briefly reviewed with specific focus on the plastic pipe system used in the experimental work reported in Section 4. This model is extremely simple, but it is believed that it captures the main dynamic effects of the pipe and the sensor, which determine the bandwidth over which leak noise can be measured in practice, and the shape of the cross-correlation function. An infinite pipe is assumed, so that there are no wave reflections at pipe discontinuities. Furthermore, basic models of the transducer response are assumed (i.e., dynamics due to internal resonances in the transducers are neglected).

As the noise propagates through the pipe, the high frequencies are attenuated because of damping in the pipe-wall and radiation of noise into the surrounding medium. Moreover the signals are further filtered by the sensors. The combined effect of the pipe and the sensors can be described by the frequency response function (FRF) between the acoustic pressure at the leak location and the sensor output (pressure, velocity or acceleration). For the sensor at Position 1 this is given by [11]:

$$
H\left(\omega, d_{1}\right)=(\mathrm{i} \omega)^{n} A_{n} e^{-\omega \beta d_{1}} e^{-\mathrm{i} \omega d_{1} / c}
$$

where $\beta$ is the attenuation factor, $n$ is related to the type of sensor being used and $A_{n}$ is a gain related to the pipe and sensor. For a hydrophone, $n=0$ so that $A_{0}=A_{\text {hyd }}$ (no units) where $A_{\text {hyd }}$, is the gain of the hydrophone measurement system. For a geophone placed on the pipe-wall, $n=1$ so 
that $A_{1}=A_{\text {geo }} a^{2} /(E h)\left(\mathrm{m}^{3} / \mathrm{N}\right)$ where $A_{\text {geo }}$ is the gain of the geophone measurement system. For an accelerometer placed on the pipe-wall, $n=2$ so that $A_{2}=A_{\text {acc }} a^{2} /(E h)$ where $A_{\text {acc }}$ is the gain of the accelerometer measurement system. At low frequencies, which is the case for leak detection in plastic pipes, the noise radiation into the surrounding medium can be neglected so that the attenuation factor is simply related to the loss in the pipe-wall and is given by [6]:

$$
\beta=\frac{1}{c_{f}} \frac{\eta B a / E h}{[1+(2 B a / E h)]^{1 / 2}}
$$

where $\eta$ is the damping in within the pipe wall, $c_{f}$ and $B$ are the free-field fluid wavespeed and the fluid bulk modulus of elasticity, respectively, and $E, a$ and $h$ are the Young's modulus of the pipe wall, the mean pipe radius, and the pipe wall thickness, respectively. The speed of leak noise propagation in the pipe (coupled motion of the fluid and in the pipe wall) is given by [6]:

$$
c=\frac{c_{f}}{(1+2 B a / E h)^{\frac{1}{2}}}
$$

Now, the cross-spectrum between the two sensor outputs, each fitted either side of a leak, is given by [9]:

$$
S_{x_{1} x_{2}}(\omega)=S_{l l}(\omega)\left|H^{*}\left(\omega, d_{1}\right) H\left(\omega, d_{2}\right)\right| e^{\mathrm{i} \omega T_{0}}
$$

where $S_{l l}(\omega)$ is the power spectral density of the acoustic pressure due to the leak at the leak location. It is clear from Examining Equations (5) and (9) it can be seen that $\left|H^{*}\left(\omega, d_{1}\right) H\left(\omega, d_{2}\right)\right|$ is an important factor in the estimation of the time delay. Using the properties in Table 1 for the high performance polyethylene (HPPE) pipe used in the experiments described in the next section, and assuming that $S_{l l}(\omega)$ is constant within the frequency range of interest [9], $\left|S_{x_{1} x_{2}}(\omega)\right| / \max \left|S_{x_{1} x_{2}}(\omega)\right|$ is plotted for a pipe with hydrophones, geophones and accelerometers in Figure 2.

Note that $\max \left|S_{x_{1} x_{2}}(\omega)\right|$ is the maximum value of $\left|S_{x_{1} x_{2}}(\omega)\right|$ within the frequency range greater than or equal to $10 \mathrm{~Hz}$. This particular frequency was chosen because in practice a high-pass filter has to be used to remove low frequency background noise, and it has been found in practice that below about $10 \mathrm{~Hz}$ the measured signals are dominated by background noise.

Table 1. Properties of the experimental test-rig.

\begin{tabular}{cc}
\hline Mean radius of the pipe & $75 \mathrm{~mm}$ \\
Pipe-wall thickness & $9.85 \mathrm{~mm}$ \\
Young's modulus & $2 \times 10^{9} \mathrm{~N} / \mathrm{m}^{2}$ \\
Loss factor of the pipe & 0.1 \\
Bulk modulus of water & $2.2 \times 10^{9} \mathrm{~N} / \mathrm{m}^{2}$ \\
Free-field wavespeed in water & $1,500 \mathrm{~m} / \mathrm{s}$ \\
Attenuation factor, $\beta$ & $1.99 \times 10^{-4} \mathrm{~N} / \mathrm{m}^{2}$ \\
Wave speed of leak noise, $c$ & $356 \mathrm{~m} / \mathrm{s}$ \\
\hline
\end{tabular}


Figure 2. $\left|S_{x_{1} x_{2}}(\omega)\right| / \max \left|S_{x_{1} x_{2}}(\omega)\right|$ for different type of sensors. Solid blue line, hydrophone; dashed green line, geophone; dotted-dashed red line, accelerometer. $\max \left|S_{x_{1} x_{2}}(\omega)\right|$ is the maximum value of $\left|S_{x_{1} x_{2}}(\omega)\right|$ within the frequency range greater than or equal to $10 \mathrm{~Hz}$.

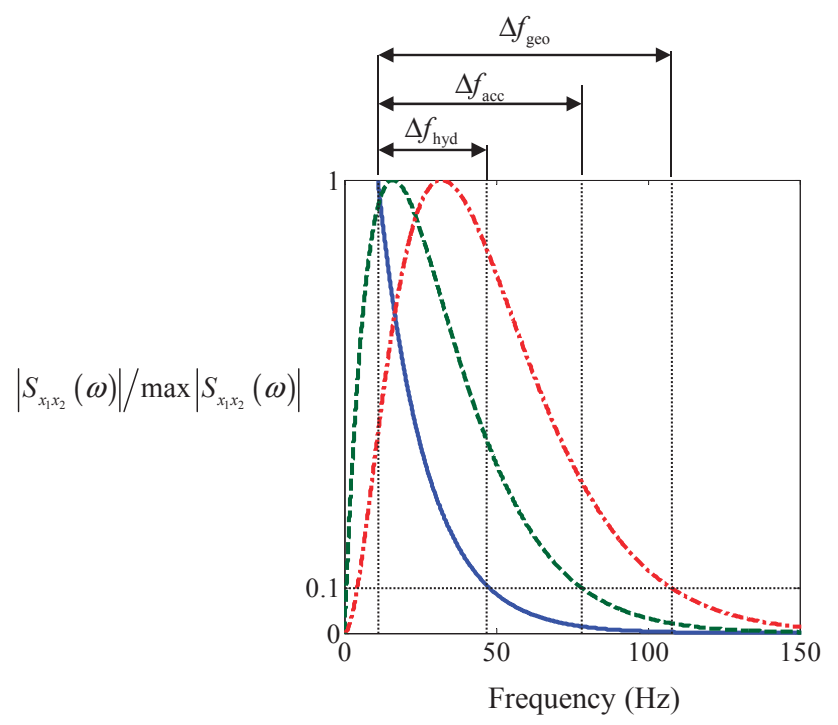

It can be seen from Figure 2 that the pipe together with the hydrophones simply acts as a low-pass filter. The peak occurs at $10 \mathrm{~Hz}$ (as it is set to zero below this frequency because of background noise, as mentioned above) and is one tenth of this value at a frequency of about $47 \mathrm{~Hz}$. For both the geophones and the accelerometers, the combined pipe-sensor system acts as a band-pass filter with peaks occurring at about $16 \mathrm{~Hz}$ and $32 \mathrm{~Hz}$, respectively. The normalized CSDs are one tenth of the peak values at frequencies of about $78 \mathrm{~Hz}$ and $108 \mathrm{~Hz}$, respectively.

Filtering the signals creates additional peaks in the cross-correlation function, which can mask the main peak due to the time delay. A measure of the height of these peaks compared to the main peak is the ratio $\Delta f / f_{c}$ [12], where $\Delta f=f_{\text {upper }}-f_{\text {lower }}$ in which $f_{\text {upper }}$ is the upper cut-off frequency and $f_{\text {lower }}$ is the lower cut-off frequency of the band-pass filter, and $f_{c}=\left(f_{\text {upper }}+f_{\text {lower }}\right) / 2$ is the central frequency of the band-pass filter. A value close to 2 (which is the maximum value that $\Delta f / f_{c}$ can have) indicates that the additional peaks are small compared to the main peak [12]. Using $10 \mathrm{~Hz}$ as the lower frequency, and (arbitrarily) choosing the upper frequency to coincide with the values of $\left|S_{x_{1} x_{2}}(\omega)\right| / \max \left|S_{x_{1} x_{2}}(\omega)\right|=0.1$ results in the values given in Table 3 below, along with the assumed values of $f_{\text {lower }}$ and $f_{\text {upper }}$. It can be seen that for the particular configuration of interest here, the best sensor to use based on this criterion should be an accelerometer. There are, of course, other criteria, and these will be discussed later in the paper. 


\section{Experiment}

\subsection{Experimental Procedure}

The experiments were conducted on a $110 \mathrm{~m}$ long bespoke pipe rig located at Blithfield reservoir in Staffordshire, UK. Some details of the pipe rig are shown in Figure 3, the properties of which are given in Table 1. Figure 3a shows a schematic of the pipe rig, in which the position of the leak and the two sensors can be seen. The end of the pipe close to Position 1 is connected to the mains water distribution pipe, which supplies water at a pressure of about 6 bar. The access points are set in concrete to provide a rigid support for the pipe connections, while the pipe sections are buried in the ground at a depth of about $0.8 \mathrm{~m}$. Three sensors (hydrophone, geophone and accelerometer) were positioned at the access points at Positions 1 and 2 which were $30 \mathrm{~m}$ and $20 \mathrm{~m}$ from the leak respectively. A photograph of one these positions is shown in Figure 3b. Figure 3c shows a schematic of the main valve, which is at each at access point, and the standpipe which was fitted to the access point where the leak was induced.

Figure 3. Details of the Blithfield pipe test-rig. (a) schematic showing the distances and the excitation/measurement positions and the leak position; (b) One of the access points and part of the instrumentation used. $\{1\}$ Hydrophone; $\{2\}$ Accelerometer; $\{3\}$ Charge amplifier; $\{4\}$ Geophone; (c) Sketch of the device used for generating the leak condition. $\{5\}$ Water distribution plastic pipe; $\{6\}$ Main valve; $\{7\}$ Standpipe; $\{8\}$ Secondary valve; $\{9\}$ Hydrant; (d) Photograph showing the leak from the standpipe.

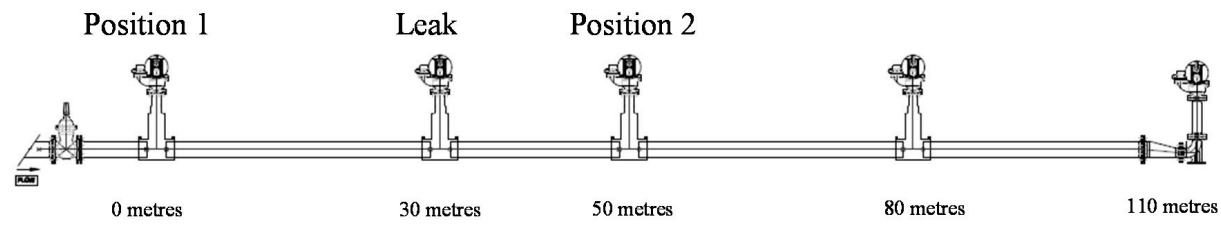

(a)

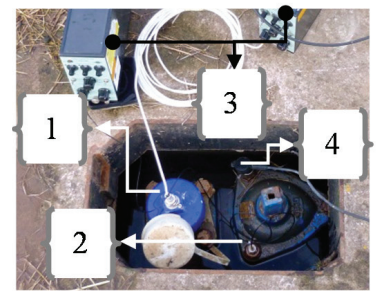

(b)

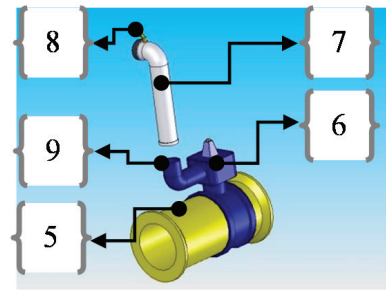

(c)

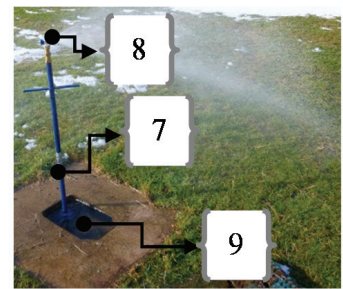

(d)

The leak was induced by opening the secondary valve and can be seen in Figure $3 \mathrm{~d}$. Although this is not a sub-surface leak it does generate a noise which is very similar to a leak below the surface, as the mechanism for leak noise is the turbulent nature of the fluid as it passes through an orifice or a leak $[17,18]$. In this work two different leak strengths were induced; a strong leak by 
opening the secondary valve fully, and a weak leak by partially closing this valve. These conditions can be seen in the photographs in Figures 4.

Figure 4. Leak induced in the pipe. (a) Strong leak; (b) Weak leak.

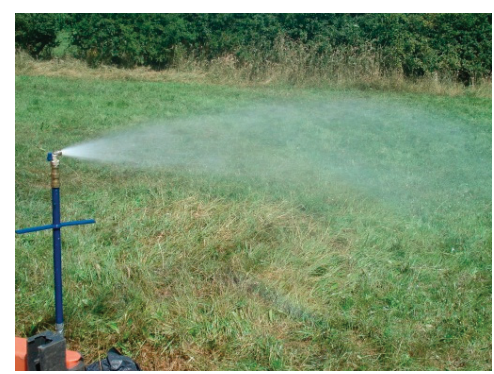

(a)

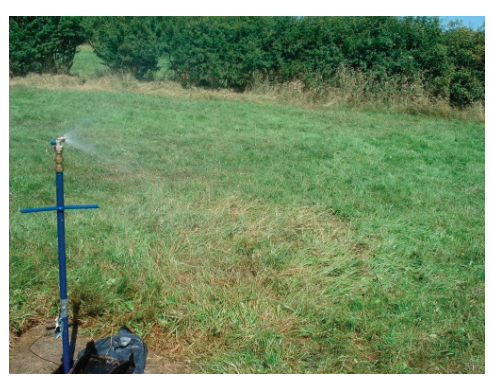

(b)

The leak noise sensed by the three transducers at Positions 1 and 2 was measured simultaneously for one minute using DATS [19]. The sampling frequency was set to $5 \mathrm{kHz}$, and a frequency resolution of $1 \mathrm{~Hz}$ was used in the subsequent spectral analysis. The details of the transducers and part of the instrumentation used in the experiment are given in Table 2. Analysis of the data collected is described in the next Section.

Table 2. Instrumentation used in the experiment.

\begin{tabular}{ccc}
\hline Device & Manufacturer & Type \\
\hline Hydrophones & Bruel and Kjaer & 8103 \\
Geophones & Ion & SM-24 \\
Accelerometers & Bruel and Kjaer & 4383 and 4384 \\
Charge Amplifiers & Bruel and Kjaer & 2635 \\
Acquisition System & Prosig & DATS \\
\hline
\end{tabular}

\subsection{Data Processing}

Prior to analysis, the data from all the sensors were passed through band-pass filters with lower and upper limits set to $10 \mathrm{~Hz}$ and $150 \mathrm{~Hz}$, respectively. As mentioned previously, a frequency of $10 \mathrm{~Hz}$ was chosen for the lower limit to remove background noise due to the environment. The upper frequency of $150 \mathrm{~Hz}$ was chosen based on an estimate of the attenuation of the leak noise signal at the frequency. The attenuation in $\mathrm{dB} / \mathrm{m}$ at frequency $\omega$ is given by $8.67 \beta \omega$ [9]. Combining this with Equation (7) results in an attenuation of about $1.6 \mathrm{~dB} / \mathrm{m}$ at $150 \mathrm{~Hz}$. Thus, at $20 \mathrm{~m}$ and $30 \mathrm{~m}$ from the leak, the attenuation in the leak noise will be about $32 \mathrm{~dB}$ and $48 \mathrm{~dB}$ at $150 \mathrm{~Hz}$, respectively. It was expected, therefore, that the signal to noise ratio at frequencies greater than $150 \mathrm{~Hz}$ would be very small. 
Figure 5. Analysis of the strong leak data from the test-rig. (a) Hydrophone; (b) Geophone; (c) Accelerometer. (i), Normalized CSD with respect to the maximum amplitude between $10 \mathrm{~Hz}$ and $150 \mathrm{~Hz}$. (ii) Coherence. (iii) Phase. (iv) Cross correlation coefficient.
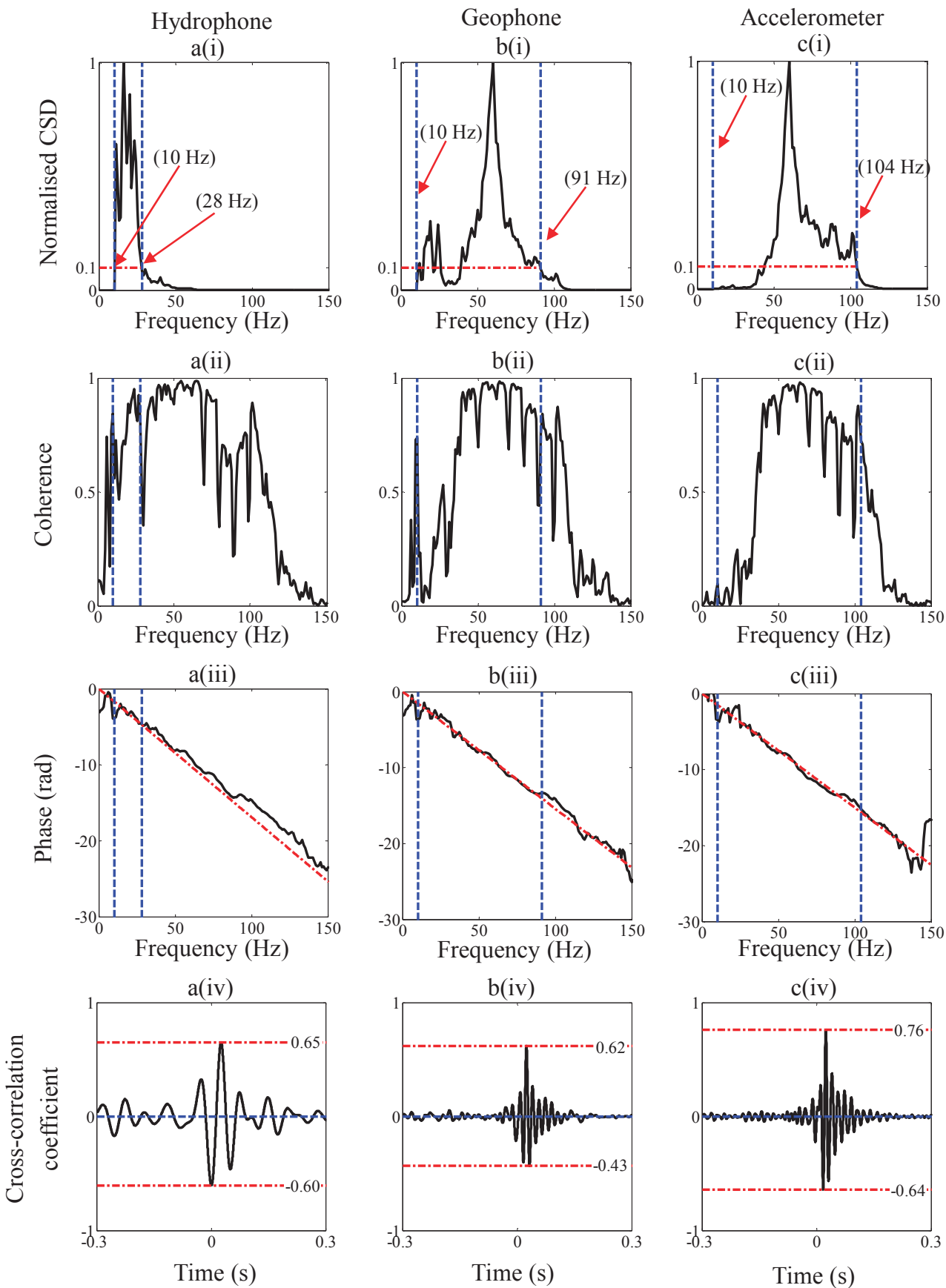
It was found that one of the signals from a geophone had a large $50 \mathrm{~Hz}$ component from the mains electrical supply that could not be removed in the field, so each geophone signal was also subsequently passed through a set of notch filters set at $50 \mathrm{~Hz}, 100 \mathrm{~Hz}$ and $150 \mathrm{~Hz}$.

Figure 5 shows the processed data from the strong leak measured using the hydrophones, geophones and accelerometers respectively. The subplots (i), (ii) and (iii) correspond to frequency domain representations, namely the normalized modulus of the CSD with respect to the maximum value between $10 \mathrm{~Hz}$ and $150 \mathrm{~Hz}$, the coherence and the phase, respectively. The subplot (iv) corresponds to the cross-correlation coefficient, from which the time delay is estimated. The key parameters extracted from the data are given in Table 3, so they can be compared directly with those predicted using the simple model.

Examining the normalized CSD in Figures $5 \mathrm{a}(\mathrm{i}), 5 \mathrm{~b}(\mathrm{i})$ and $5 \mathrm{c}(\mathrm{i})$, it can be seen that the frequency range with a lower frequency of $10 \mathrm{~Hz}$ and an upper frequency given by when the normalized CSD is equal to 0.1 , is small $(10 \mathrm{~Hz}-28 \mathrm{~Hz})$ for the hydrophone measured data compared to the geophone $(10 \mathrm{~Hz}-91 \mathrm{~Hz})$ and accelerometer $(10 \mathrm{~Hz}-104 \mathrm{~Hz})$ measured data. These frequency ranges are marked on the figures for clarity. As discussed in Section 3 this band-pass filtering feature is partly due to the combined effects of the pipe and the sensors.

By examining Figures 5a(ii), 5b(ii) and 5c(ii), it can be seen that there is reasonably good coherence in all three cases over a wide frequency range. The unwrapped phase spectra are shown in Figures 5a(iii), 5b(iii) and 5c(iii). It can be seen that although there is approximately straight line behavior over a relatively wide frequency range, there are some deviations from this behavior. These could be due to reflections at low frequencies due to discontinuities in the pipe [14], and the dynamic behavior of the system at higher frequencies [12]. As discussed in Section 2, the time delay is given by the slope of the phase gradient given by a weighted least squares fit to the phase (also shown in the figures), where the weighting factor is the modulus of the cross-spectrum between the measured signals. This is clearly affected by the type of sensor used as seen in Figures 5a(i), 5b(i) and 5c(i), which determines the effective bandwidth of the data used in the time delay calculation of each case. The cross-correlation coefficients calculated from the time series from the three types of sensors are shown in Figures $5 \mathrm{a}$ (iv), 5b(iv) and 5c(iv). The time delays corresponding to the peaks in these graphs are $25.8 \mathrm{~ms}$ for hydrophone measured data, $24.4 \mathrm{~ms}$ for geophone measured data and $24 \mathrm{~ms}$ for accelerometer measured data. Using these data together with Equation (1) and assuming a wavespeed of $356 \mathrm{~m} / \mathrm{s}$ given in Table 1 results in an estimated distance $d_{2}$ of $20.41 \mathrm{~m}, 20.66 \mathrm{~m}$ and $20.72 \mathrm{~m}$ for hydrophone, geophone and accelerometer measured data respectively. These data are also given in Table 3 for ease of reference.

Table 3. Key parameters determined from the leak data (theory and experiment (strong leak)).

\begin{tabular}{lcccc|cccc}
\hline \multirow{5}{*}{ Sensor Type } & $f_{\text {lower }}$ & $f_{\text {upper }}$ & $\frac{\Delta f}{f_{c}}$ & $d_{2}$ & $f_{\text {lower }}$ & $f_{\text {upper }}$ & $\frac{\Delta f}{f_{c}}$ & $d_{2}$ \\
& $(\mathrm{~Hz})$ & $(\mathrm{Hz})$ & $(\mathrm{m})$ & $(\mathrm{Hz})$ & 0.95 & 20.41 \\
& 10 & 47 & 1.30 & 20 & 10 & 28 & 0.95 \\
Hydrophone & 10 & 78 & 1.55 & 20 & 10 & 91 & 1.6 & 20.66 \\
Geophone & 10 & 108 & 1.66 & 20 & 10 & 104 & 1.65 & 20.72 \\
\hline
\end{tabular}


Figure 6. Analysis of the weak leak data from the test-rig. (a) Hydrophone; (b) Geophone; (c) Accelerometer. (i), Normalized CSD with respect to the maximum amplitude between $10 \mathrm{~Hz}$ and $150 \mathrm{~Hz}$. (ii) Coherence. (iii) Phase. (iv) Cross correlation coefficient.
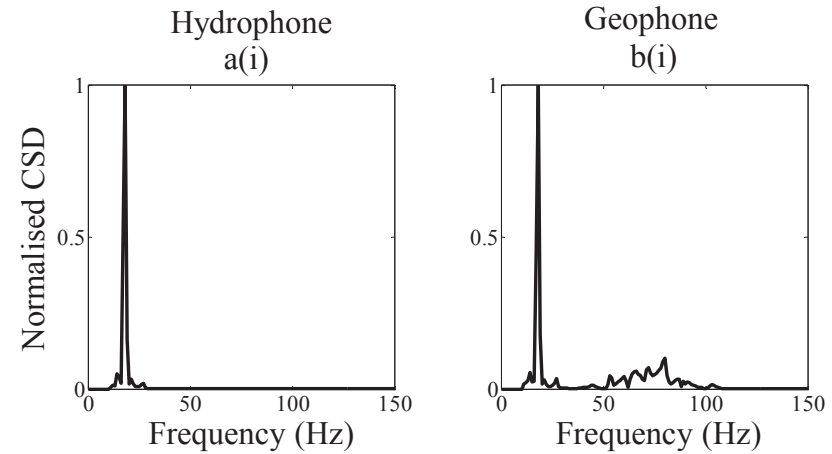

Accelerometer c(i)
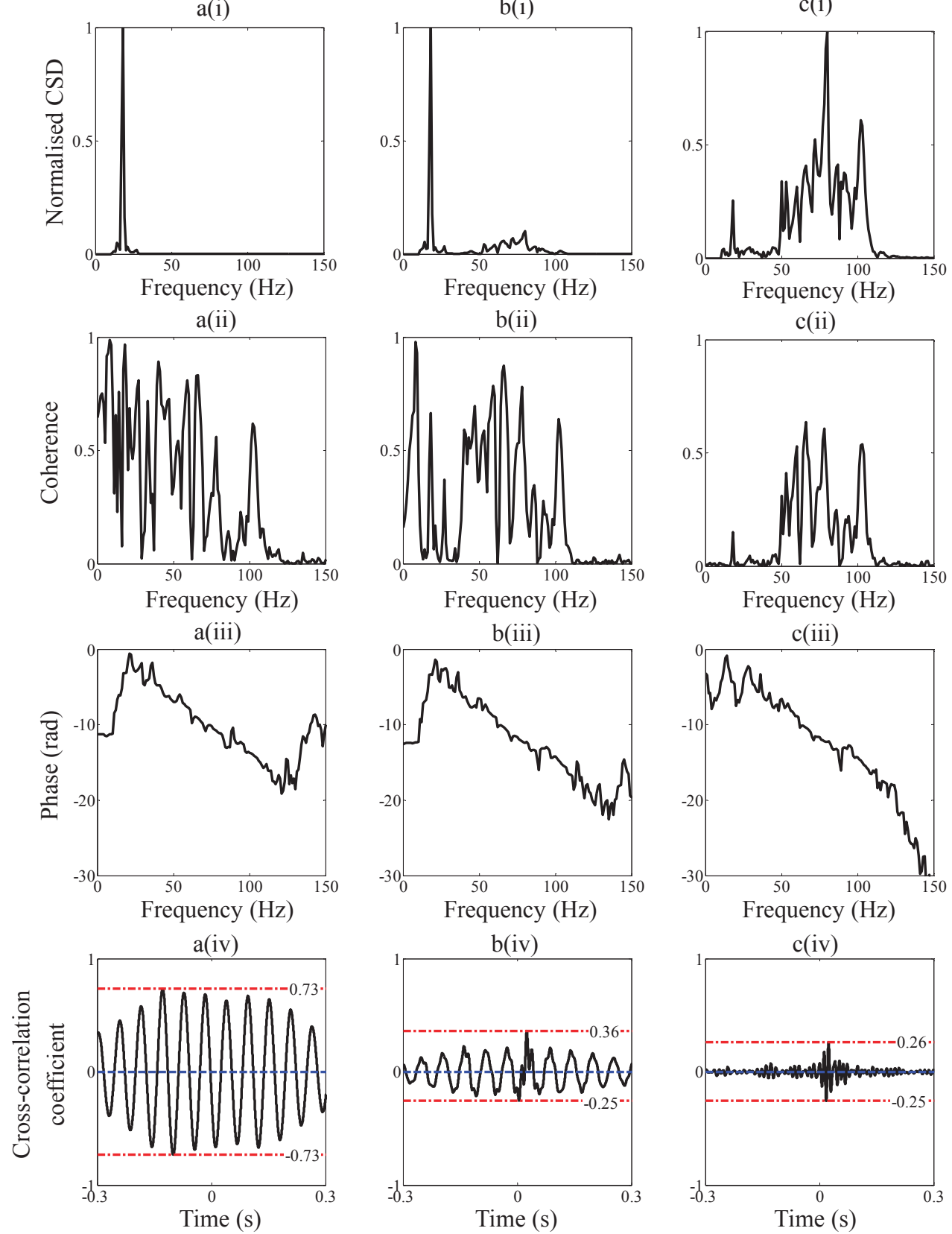
Figure 6 shows the processed data corresponding to the weak leak. Examining the normalized CSD in Figures 6a(i), 6b(i) and 6c(i), it can be seen that much energy is contained in a narrow band of frequencies between $18 \mathrm{~Hz}$ and $22 \mathrm{~Hz}$, especially in the hydrophone and geophone data. This was not due to the leak, but was thought to be related to water flow in the mains pipe which fed the test rig (so-called out-of-bracket noise). The reason why the accelerometer data in Figure $6 c(i)$ was not affected as much by this noise is because of the additional high-pass filter effect due to the sensors which is predicted by the model of the pipe-sensor system (see Figure 2).

By comparing the coherence in the weak and the strong leak cases it can be seen that the coherence was much reduced in the weak leak case resulting in a much lower signal to noise ratio, which is to be expected. In the unwrapped phase spectra for the hydrophone and the geophone a phase with positive gradient can be seen at low frequencies. This is related to the out-of-bracket noise rather than the leak noise. The cross-correlation coefficients are shown in Figures 6a(iv), $6 \mathrm{~b}$ (iv) and $6 \mathrm{c}$ (iv). It is evident that only the geophone and the accelerometer are effective at detecting the leak in this case, with peaks occurring at $24.8 \mathrm{~ms}$ and $24 \mathrm{~ms}$ respectively. The quality of the correlation is much diminished, however, compared to the strong leak case.

\section{Discussion}

The key parameters extracted from the simple model and the experimental test for the strong leak are summarized in Table 3. The time delays are extracted directly from the experimental data and these need to be processed to give the leak position. To do this the speed at which the noise propagates needs to be known or estimated. This could be estimated from measurements using the three different sensors, but different results would be obtained in each case [12], as mentioned previously in Section 2. Thus, to make a meaningful comparison between the three sensors, the time delay estimates should be considered. They cannot, however, be compared against a reference value as this is unknown (and would be unknown for any practical case). To simplify the comparison, a nominal wave speed is calculated using Equation (8) (given in Table 1), so that the distance from Position 2 can be calculated using Equation (1). Of course this cannot be taken as a true estimate of the position of the leak but it does facilitate a comparison between the sensors (and the wave speed is mainly estimated this way in practice).

It can be seen in Table 3 that the distance estimated using the three different sensor types varies by less than $2 \%$. This is to be expected, because for a plastic pipe with dimensions of those in the test rig, the acoustic behavior of the fluid is well-coupled to the vibration of the pipe [6]. Although it appears that the hydrophone data gives the most accurate estimate, this will change if the speed of noise propagation changes. It can be seen from Equation (8) that the speed of noise propagation is dependent on the Young's modulus of the pipe which varies with temperature and this was not known precisely. To illustrate how sensitive the predictions are on the speed of noise propagation, Equation (1) is combined with Equation (8), and the estimated leak position is plotted as a function of the Young's modulus of the pipe. This is shown in Figure 7, using the three time delays estimated from the measured data. It can be seen that if the Young's modulus of the pipe was about $2.8 \times 10^{9} \mathrm{~N} / \mathrm{m}^{2}$ corresponding to a wave speed of about $416 \mathrm{~m} / \mathrm{s}$ then the geophone and accelerometer data would give a better prediction of the location of the leak. Almeida [12], 
measured the wavespeed in the Blithfield pipe-rig at different periods over 3 years using accelerometers. He found that the wavespeed changes drastically from season to season, varying from $350 \mathrm{~m} / \mathrm{s}$ to $420 \mathrm{~m} / \mathrm{s}$. Thus, it is not possible to say which sensor gives the most accurate prediction of leak location (this might be possible only in the most controlled laboratory conditions, which would not reflect the real situation in the field). It is possible to state, however, that all sensors were able to detect the leak and provide estimates for the leak location in situations in which the signal to noise ratio is not too low that are consistent with the uncertainty of the wave speed estimate. Moreover, the filtering effects of the pipe-sensor system are in qualitative agreement with the predictions from the simple model.

Figure 7. Estimate of the leak position as a function of the Young's modulus of the pipe calculated using different types of sensors. solid blue line, hydrophone; dotted green line, geophone; dashed red line, accelerometer.

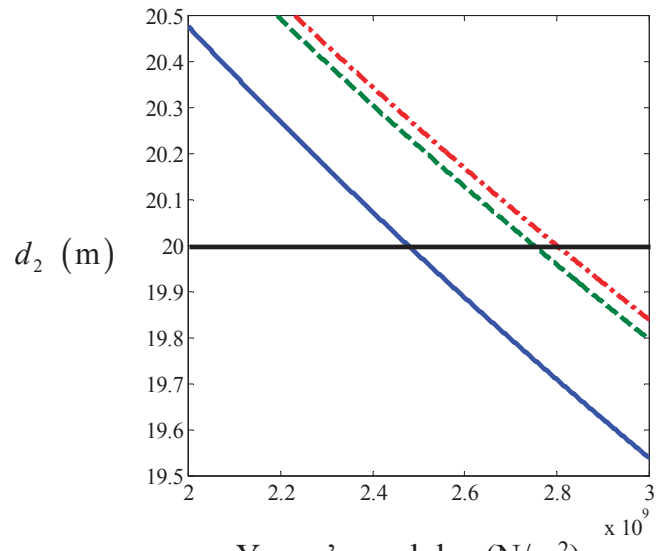

Young's modulus $\left(\mathrm{N} / \mathrm{m}^{2}\right)$

The second parameter that gives an indication of the prominence of the peak compared to minor peaks surrounding it in the cross-correlation function is the ratio $\Delta f / f_{c}$ as discussed in Section 3. This is heavily dependent on the sensor type and leak strength. For strong leak data, it can be seen that, because the geophone and accelerometer amplify the high frequency components of the leak noise in the measured signals, the bandwidth is increased, and hence $\Delta f / f_{c}$ is larger for these sensors compared to the hydrophone. The theoretical values of this quantity together with the experimental values from the strong leak are given in Table 3. It can be seen that for the geophone measured data, and in particular the accelerometer measured data, the theoretical and experimental values are very similar. There is a discrepancy with the hydrophone measured data, with the higher frequency components being attenuated at a much greater rate than that predicted as can be seen by comparing Figures 2 and $4 \mathrm{a}(\mathrm{i})$, thus limiting the effective bandwidth of this data. The reason for this is uncertain, but could be due to additional acoustic behavior of the system which was not captured by the simple analytical model. Thus, in the particular situation where the leak is considered strong, the most effective sensors for use in leak detection would be either geophones or accelerometers. For the weak leak case considered in this paper, the ratio $\Delta f / f_{c}$ for the hydrophone and geophone 
are significantly reduced when compared with the strong leak case. This can be seen by comparing the values for the weak leak given in Table 4, with those for the strong leak given in Table 3 . As mentioned in Section 3, the value of $\Delta f / f_{c}$ is measure of the shape of the cross-correlation function and hence the prominence of the peak in this function which is related to the leak. It is clear that the peak is not discernible in the hydrophone-measured data, and it is much diminished in the geophone-measured for the weak leak compared to the strong leak.

Table 4. Key parameters determined from the weak leak data (experiment only).

\begin{tabular}{|c|c|c|c|c|}
\hline \multirow[b]{2}{*}{ Sensor Type } & \multicolumn{4}{|c|}{ Experiment } \\
\hline & $\begin{array}{l}f_{\text {lower }} \\
(\mathrm{Hz})\end{array}$ & $\begin{array}{l}f_{\text {upper }} \\
(\mathrm{Hz})\end{array}$ & $\frac{\Delta f}{f_{c}}$ & $\begin{array}{l}d_{2} \\
(\mathrm{~m})\end{array}$ \\
\hline Hydrophone & 10 & 19 & 0.62 & - \\
\hline Geophone & 10 & 19 & 0.62 & 20.58 \\
\hline Accelerometer & 10 & 87 & 1.59 & 20.73 \\
\hline
\end{tabular}

In some situations, the structural sensors, accelerometers and geophones can pick up a significant of low frequency background noise and thus it is not possible for them to sense low frequency leak noise. This becomes particularly troublesome when the attenuation factor is large or the distance between the sensors and the leak is large as the high frequencies are heavily attenuated resulting in a very small frequency range where there is leak noise in the measured data. As can be seen in Equation (7) the attenuation factor is dependent upon the damping in the pipe wall which is governed by the pipe material, and the size and thickness of the pipe. Thus, a larger diameter pipe has a higher attenuation factor than a smaller diameter pipe if the pipe material remains the same. In some situations, therefore, it is desirable to use hydrophones as they are more sensitive to low frequencies compared to the structural sensors considered. It should be noted, however, that hydrophones have the disadvantage of being invasive.

\section{Conclusions}

This paper has described an experimental study to investigate the combined filtering effects of the sensors and pipe on a bespoke plastic water pipe test-rig for the purposes of leak detection. The sensors considered were hydrophones, geophones and accelerometers. Two different leaks were considered; a strong leak where the signal to ratio was high, and a much weaker leak strength where this was not the case. It was found that the experimental results for a strong leak were broadly in agreement with those predicted from a simple analytical model proposed in $[10,11]$. The main features are that the hydrophone-pipe system is more sensitive at low frequencies with the pipe acting as a low-pass filter, and the geophone and the accelerometer combined with the pipe effect results in band-pass filter behavior for these systems.

For the strong leak all three sensors were capable of detecting and locating the leak. However the weak leak could not be detected by the hydrophone sensors, as there was noise in a narrow range of low frequencies that were not related to the leak and dominated the signals from these sensors. This also affected the geophone data to some extent and the accelerometer data much 
less so, which meant that only the accelerometer could clearly detect and locate the leak in this case. The band-pass filter effect of the pipe and these sensors is the reason why this occurred.

A quality measure for the data was also presented, which is the ratio of the bandwidth over which the analysis is carried out divided by the centre frequency of this bandwidth. Based on this metric, the accelerometer was found to be the best sensor to use for the test rig described in this paper. However, if the attenuation factor in the pipe is large, either because of damping or because of pipe geometry, then it may be desirable to use hydrophones even though they are invasive sensors. It was also demonstrated that accurate location of the leak position is heavily dependent upon the precision with which the leak noise propagation speed is known, and for a plastic pipe, this is independent of the type of sensor being used.

\section{Acknowledgments}

The authors would like to acknowledge the technical and financial support provided by South Staffs Water plc and the partial financial support provided by FEPISA.

\section{Conflict of Interest}

The authors declare no conflict of interest.

\section{References}

1. McMahon, W.; Burtwell, M.H.; Evans, M. Minimising Street Works Disruption: The Real Costs of Street Works to the Utility Industry and Society. Techical Report 05/WM/12/8, UK Water Industry Research: London, UK, 2005.

2. Muggleton, J.M.; Brennan, M.J. The use of acoustics in the water industry. Water Sewerage J. 2012, 4, 35-36.

3. Muggleton, J.M.; Brennan, M.J.; Gao, Y. Determing the location of buried plastic water pipes from measurements of ground surface vibration. J. Appl. Geophys. 2011, 75, 54-61.

4. Romanova, A.; Bin Ali, M.T.; Horoshenkov, K.V. A non-intrusive acoustic method of sewer pipe survey-A novel, inexpensive and rapid primary survey technique. AWA Water J. Asset Manag. 2011, 38, 103-105.

5. Fuchs, H.V.; Riehle, R. Ten years of experience with leak detection by acoustic signals analysis. Appl. Acoust. 1991, 33, 1-19.

6. Muggleton, J.M.; Brennan, M.J.; Pinnington, R.J. Wavenumber prediction of waves in buried pipes for water leak detection. J. Sound Vib. 2002, 249, 939-954.

7. Muggleton, J.M.; Brennan, M.J.; Linford, P.W. Axisymmetric wave propagation in fluid-filled pipes: Wavenumber measurements in-vacuo and buried pipes. J. Sound Vib. 2004, 270, 171-190.

8. Hunaidi, O.; Chu, W.T. Acoustical characteristics of leak signals in plastic water distribution pipes. Appl. Acoust. 1999, 58, 235-254.

9. Gao, Y.; Brennan, M.J; Joseph, P.F.; Muggleton, J.M.; Hunaidi, O. A model of the correlation function of leak noise in buried plastic pipes. J. Sound Vib. 2004, 277, 133-148. 
10. Muggleton, J.M.; Brennan, M.J. Axisymmetric wave propagation in buried, fluid-filled pipes: Effects of the wall discontinuities. J. Sound Vib. 2005, 281, 849-867.

11. Gao, Y.; Brennan, M.J; Joseph, P.F.; Muggleton, J.M.; Hunaidi, O. On the selection of acoustic/vibration sensors for leak detection in plastic water pipes. J. Sound Vib. 2005, 283, 927-941.

12. Almeida, F.C.L. Improved Acoustic Methods for Leak Detection in Buried Plastic Water Distribution Pipes. Ph.D. Thesis, University of Southampton: Southampton, UK, 2013.

13. Brennan, M.J.; Gao, Y.; Joseph, P.F. On the relationship between time and frequency domain methods in time delay estimation for leak detection in water distribution pipes. J. Sound Vib. 2007, 304, 213-223.

14. Brennan, M.J.; Joseph, P.F.; Gao, Y. On the effects of reflections on time delay estimation for leak detection in buried plastic water pipes. J. Sound Vib. 2009, 325, 649-663.

15. Bendat, J.S.; Piersol, A.G. Random Data: Analysis and Measurement Procedures, 3rd ed.; Wiley-Interscience: New York, NY, USA, 2000.

16. Knapp, C.H.; Carter, G.C. The generalized correlation method for estimation of time delay. J. Sound Vib. 1976, 24, 320-327.

17. Papastefanou, A.S.; Joseph, P.F.; Brennan, M.J. Experimental investigation into the characteristics of in-pipe leak noise in plastic water filled pipes. Acta. Acust. United Acust. 2012, 98, 847-856.

18. Ben-Mansour, R.; Habib, M.A.; Khalifa, A.; Youcef-Toumi, K.; Chatzigeorgiou, D. Computational fluid dynamic simulation of small leaks in water pipelines for direct leak pressure transduction. Comput. Fluid. 2012, 57, 110-123.

19. Prosig Webpage. Available online: www.prosig.com/noise-vibration-measurementsystems/dats/ (accessed on 6 November 2013). 


\title{
Application of Morphological Segmentation to Leaking Defect Detection in Sewer Pipelines
}

\section{Tung-Ching Su and Ming-Der Yang}

\begin{abstract}
As one of major underground pipelines, sewerage is an important infrastructure in any modern city. The most common problem occurring in sewerage is leaking, whose position and failure level is typically identified through closed circuit television (CCTV) inspection in order to facilitate rehabilitation process. This paper proposes a novel method of computer vision, morphological segmentation based on edge detection (MSED), to assist inspectors in detecting pipeline defects in CCTV inspection images. In addition to MSED, other mathematical morphology-based image segmentation methods, including opening top-hat operation (OTHO) and closing bottom-hat operation ( $\mathrm{CBHO})$, were also applied to the defect detection in vitrified clay sewer pipelines. The CCTV inspection images of the sewer system in the 9th district, Taichung City, Taiwan were selected as the experimental materials. The segmentation results demonstrate that MSED and OTHO are useful for the detection of cracks and open joints, respectively, which are the typical leakage defects found in sewer pipelines.
\end{abstract}

Reprinted from Sensors. Cite as: Su, T.-C.; Yang, M.-D. Application of Morphological Segmentation to Leaking Defect Detection in Sewer Pipelines. Sensors 2014, 14, 8686-8704.

\section{Introduction}

Sewerage is an important infrastructure in modern cities so the pipeline authorities in Taiwan have been making considerable efforts to construct sewer systems. Nowadays, the percentage of sewer system coverage in Taiwan is reported to be $32.9 \%$ [1]. The most common problem occurring in sewer pipelines is leakage, whose position and failure level can be identified through image inspection in order to facilitate the rehabilitation process. Distributing fiber optics is an efficient way to detect and localize leakages along pipelines by monitoring pressure or temperature differences. Fiber optic systems have been demonstrated useful in monitoring leakage of buried pipelines, such as oil and gas pipelines [2-5], but sewerage consists of gravity pipelines rather than pressure pipelines so fiber optics are inapplicable for leakage detection of sewerage.

Sewer rehabilitation involving sewer inspection, assessment of structural conditions, computation of structural condition grades, and determination of rehabilitation methods and substitution materials is necessary for the buried sewer pipes to maintain the designed drainage capability [6-10]. At present, closed circuit television (CCTV) is the most popular equipment for sewer inspection because of its low cost compared with sewer scanner evaluation technology (SSET) cameras, ground piercing radar (GPR), sonar, and infrared thermograph [11-14]. However, human fatigue and subjectivity, and time-consumption for engineers may be barriers for detecting and diagnosing defects in sewer pipelines via CCTV images due to the great number of images that must be inspected. Recently, image processing and artificial intelligence techniques were applied as diagnostic systems to assist engineers in interpreting sewer pipe defects in inspection images [15-20]. 
Among the image processing techniques, mathematical morphology-based image segmentation has been extensively applied in pattern recognition research [18-25]. Erosion and dilation are two basic operators of morphological segmentation, and are usually operated in tandem for the image enhancement of objects of interest [26]. During the erosion and dilation operations, the computation of mathematical logic, including intersection and union, is simultaneously introduced into the morphology analysis. A determination of structural element (SE) is necessary before the operation of erosion and dilation. The size and shape of SE depend on the objects of interest. For example, linear SE was adopted in the operation of erosion and dilation for segmenting cracks from CCTV images [15].

Erosion and dilation with linear structural elements were performed to segment vessel-like patterns, which are very common in medical images [21]. Then, a cross-curvature evaluation was implemented to differentiate vessels from analogous background patterns. Mathematical morphology and curvature evaluation were also employed in detecting crack patterns in pipeline images [18]. Sinha and Fieguth used opening top-hat operation (OTHO), in which firstly erosion followed by dilation (called image opening) was applied to an SSET inspection image, secondly the opening operated image was subtracted from its SSET inspection image (called top-hat operation), and finally the top-hat operated image was transferred into a binary one by Otsu technique to segment pipeline defects, including cracks, holes/joints, laterals, and pipe collapse, in sewer pipelines [19]. However, environmental noise or poor image quality would deteriorate the performance of OTHO so as to hamper the pipeline defect detection. To effectively and correctly detect sewer pipeline defects, this paper presents a novel algorithm, morphological segmentation based on edge detection (MSED), to segment sewer pipeline defects from CCTV inspection images. Edge detection is considered as an important pre-processing step in image segmentation [27-29]. Based on edge detection, MSED attempts to search complete and correct image regions of sewer pipeline defects in CCTV images.

\section{Methodology}

In addition to OTHO, closing bottom-hat operation (CBHO), which is a dual operation of OTHO, was also employed to detect pipeline defects in this research. Figure 1 shows the schematic outline of this research. Edge detection and MSED algorithm are involved in the MSED method, whereas image opening, image closing, image subtraction, and Otsu's thresholding are involved in the OTHO or CBHO methods. During sewer inspection, both pipeline defects and environmental noise are imaged by the CCTV recorder so complete and correct segmentation of pipeline defects from the CCTV inspection images becomes difficult [10]. Environmental noises, such as shadows and stains resulting from inappropriate imaging condition of the CCTV robot and aged pipes, respectively, are different from image noises, such as impulsive noise, Gaussian noise, speckle noise, and periodic noise. However, sometimes stains inside pipeline shows an analogy of impulsive noise in CCTV images. Median filter, one of the smoothing filters, is well suited to remove impulsive noise [30]. Before the operation of OTHO, CBHO or MSED, a median filtering of a $5 \times 5$ window is adopted to reduce rather than remove the impact of the environmental noises on the image segmentation. The performance of the environmental noise reduction by OTHO and MSED had 
been demonstrated in the literature of Su et al. [10], who indicated that MSED outperforms OTHO. Nevertheless, the correctness and completeness of the pipeline defect segmentation by OTHO and MSED have not been estimated. After the image segmentation by OTHO, CBHO or MSED, the accuracy of the pipeline defect detection was estimated by comparing the segmentation result with the manual interpretation data.

Figure 1. Research scheme.

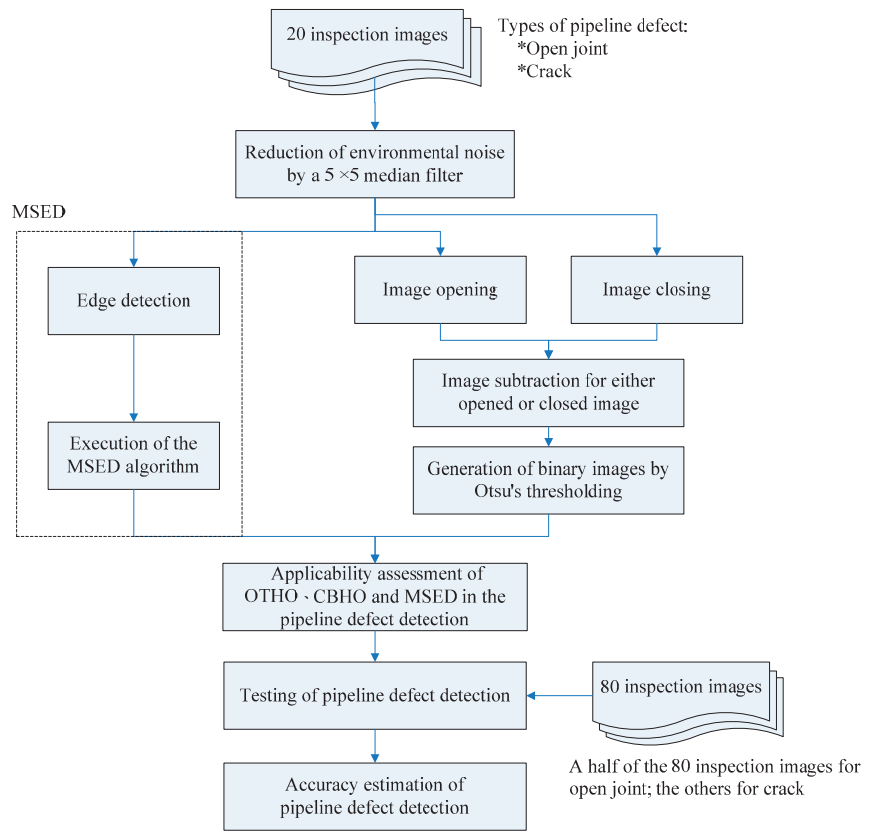

\subsection{Median Filtering}

Digital numbers of pixels possess the characteristic of signal variance in a two-dimensional space. If significant differences appear between the digital numbers, the image details, including objects and noise, can be detected by proper image processing techniques. Low-pass filters, including mean filter, Gaussian filter, and median filter, can be applied for noise removal [30-32]. Regarded as an image smoother, low-pass filtering replaces each digital number in an input image by the mean of its neighbors and itself. Mean filtering has the capability of effectively removing signals with great variance, but sometimes a median filter outperforms a mean filter in the sense of preserving useful image details [30]. In this paper, a median filter was applied to the inspected images before image segmentation. The size and shape of a kernel must be determined prior to median filtering. A square kernel consisting of $M \times M$ elements is usually applied to median filtering. Generally, $M$ must be an odd number above or equal to 3 ( $M=5$ was adopted in this paper) so that kernel has the only central element to be filtered [33]. 


\subsection{Opening Top-Hat Operation (OTHO)}

Image segmentation can be performed by the OTHO method to produce a binary image, in which the image regions consists of white pixels expressing the objects of interest, i.e., pipeline defects in this research, and black pixels denoting the background environment. Considering a structuring element as a parameter to morphological operation, the light and dark portions in an image can be reshaped or morphed in various ways [19], such dilation and erosion as two basic morphological operations [34]. Sets $\mathbf{M}$ and $\mathbf{S}$ represent a median filtered image consisting of pixels $p(x, y)$ and a structuring element, respectively:

$$
\begin{gathered}
\mathbf{M}=\{(x, y) \mid p(x, y)\} \\
\mathbf{S}=\{(x, y) \mid(x, y) \text { in structuring element }\}
\end{gathered}
$$

The dilation of $\mathbf{M}$ by $\mathbf{S}$, denoted $\mathbf{M} \oplus \mathbf{S}$, is the union of all pixels in $\mathbf{M}$ surrounded by the shape of $\mathbf{S}$ and defined as:

$$
\mathbf{M} \oplus \mathbf{S}=\{m+s \mid \text { for all } m \in \mathbf{M} \text { and } s \in \mathbf{S}\}
$$

Similarly, the erosion of $\mathbf{M}$ by $\mathbf{S}$, denoted $\mathbf{M} \Theta \mathbf{S}$, removes all pixels within a "distance" $\mathbf{S}$ from the edge of $\mathbf{M}$ and is defined as:

$$
\mathbf{M} \Theta \mathbf{S}=\{m \mid s+m \in \mathbf{M} \text { for every } s \in \mathbf{S}\}
$$

Based on Equations (3) and (4), the opening operation is defined as:

$$
\mathbf{M} \circ \mathbf{S}=(\mathbf{M} \Theta \mathbf{S}) \oplus \mathbf{S}
$$

Image regions rarely relative to the structuring element are removed by the opening operation while preserving image regions greater than structuring elements [19]. Based on Equation (5), OTHO is defined as:

$$
\mathrm{OTHO}_{\mathbf{M}} \cdot \mathbf{S}=\mathbf{M}-(\mathbf{M} \Theta \mathbf{S}) \oplus \mathbf{S}
$$

Equation (6) can be regarded as an opening operated image extracted from the median filtered one, and is also called image subtraction or top-hat operation [32]. Based on Equation (1) through Equation (6), the structural element $\mathbf{S}$, which is a matrix consisting of only 0 s and $1 \mathrm{~s}$, has a great impact on the performance of OTHO. In the matrix, the eight-neighbor pixels of $1 \mathrm{~s}$ can be assigned as of arbitrary shape and size. Diamond, disk, line, rectangular, and square are the common structuring elements; nevertheless, line structuring elements are not frequently adapted due to its ability of detecting a single border [35]. In inspection images, open joints looks like illuminated ring-like objects, but cracks usually present an irregular or linear pattern. Due to the great difference between the patterns of open joints and cracks, a disk SE with a radius of 3 pixels was adopted to assist OTHO in morphologically probing the pipeline defects. 


\subsection{Closing Bottom-Hat Operation (CBHO)}

$\mathrm{CBHO}$ is the dual operation of OTHO. Image dilation and erosion are two basic operations of image opening and closing, whereas image closing is an inverted operation of image opening and defined as:

$$
\mathbf{M} \cdot \mathbf{S}=(\mathbf{M} \oplus \mathbf{S}) \Theta \mathbf{S}
$$

Based on Equation (7), $\mathrm{CBHO}$ is defined as:

$$
\mathrm{CBHO}_{\mathbf{M}} \cdot \mathbf{s}=(\mathbf{M} \oplus \mathbf{S}) \Theta \mathbf{S}-\mathbf{M}
$$

\subsection{Morphological Segmentation Based on Edge Detection (MSED)}

Another image segmentation method, MSED, was employed to segment the pipeline defects from inspection images. Based on edge detection results, the MSED algorithm attempts to transform black pixels between two detected edges into white pixels. Edge detection technique and the MSED algorithm are introduced as follows.

\subsubsection{Edge Detection}

Edge detection is the most common approach for detecting meaningful discontinuities in gray level [36]. Edge detector, i.e., a high-pass filter, allows high-frequency components but excludes low-frequency ones to pass [30]. In this research, the Sobel first derivative edge detector was taken into consideration for the edge detection of the pipeline defects in the median filtered image $\mathbf{M}$.

\subsubsection{The MSED Algorithm}

A hybrid approach, integrating mathematical morphological edge detection, contour-based image segmentation, and trajectory estimation, was presented to track multiple objects in video frames [26]. Region growing and merging were introduced into the contour-based image segmentation algorithm, so several initial growing seeds are needed for searching the morphologies of the contoured objects. In the MSED algorithm, firstly edge detection generates a binary image (or logical image), in which $1 \mathrm{~s}$ and $0 \mathrm{~s}$ represent the border of pipeline defects and background environment, respectively. Secondly, the MSED algorithm applies region growing to the morphological segmentation of pipeline defect based on the binary image so that initial growing seeds are unneeded. A brief illustration of the MSED algorithm can be referred to the literature of $\mathrm{Su}$ et al. [10], and is encoded in Matlab as follows. In the following operation, dlim is used to survey the minimum distance between two border pixels in either row or column direction. Once the minimum distance is determined, the interval pixels (background pixels) are defined as the pipeline defect pixels:

$$
\begin{aligned}
& \text { For } d=2: d_{\text {lim }} \quad \% d_{\text {lim }} \text { is an integer, and } d=2,3,4, \ldots, d_{\text {lim }} \text { are tested. } \\
& \text { For } i=1: m \quad \% m \text { and } n \text { denote the pixel number of the image region in the row and col. } \\
& \text { For } j=1: n-d
\end{aligned}
$$


If (image $(i, j)==1 \&$ image $(i, j+d)==1) \%$ image $(i, j)$ is edge detection binary image. image $(i, j+1: j+d-1)=1$;

End

End

End

For $j=1: n$

For $i=1: m-d$

If (image $(i, j)==1 \&$ image $(i+d, j)==1)$

image $(i+1: i+d-1, j)=1$;

End

End

End

End

\subsection{Accuracy Estimation of Pipeline Defect Detection}

To verify the three segmentation methods in pipeline defect detection, the accuracy indices, including completeness (Compl), correctness (Corr), and quality, are introduced as [18]:

$$
\begin{gathered}
\text { Compl }=\left(S_{e} \cap M_{s}\right) / M_{s}, \\
\text { Corr }=\left(S_{e} \cap M_{s}\right) / S_{e}, \\
\text { Quality }=\left(\text { Compl }^{*} \text { Corr }\right) /\left(C o m p l-\text { Compl }^{*} \text { Corr }+ \text { Corr }\right),
\end{gathered}
$$

where $S_{e}$ is the segmentation by OTHO, CBHO, or MSED methods; $M_{s}$ expresses the image regions of true pipeline defects interpreted by experts a priori.

\section{Results and Discussion}

The vitrified clay sewer pipelines in Taichung City, which is the biggest city in central Taiwan, were inspected by a CCTV camera mounted on a robot. The video streams are in MPEG at 30 frames per second. A manhole to manhole distance was scheduled as an inspection unit and required about $10 \mathrm{~min}$ for CCTV shooting. Thousands frames of inspection images were recorded in each video stream. Figure 2 shows the CCTV robot and its corresponding monitoring system inside the vehicle. The CCTV robot is connected with the vehicle on the ground by a power cable. The power cable provides two utilities for the inspection task, including delivering image signal back to the vehicle and pulling the CCTV robot back while the inspection task of a pipeline length (a distance between two manholes) is finished. Moreover, the CCTV camera can be rotated along $x$ and $z$ axes if necessary (see Figure 2b). This paper used the software_VirtualDub, which can rank images in a row according to imaged time, to capture 100 frames of inspection images from the video streams as experimental materials, and assessed the applicability of the three segmentation methods for pipeline defect detection. 
Figure 2. (a) CCTV robot. (b) Illustration of the CCTV camera rotating along the $x$ and $z$ axes. (c) The monitoring system of the CCTV robot.

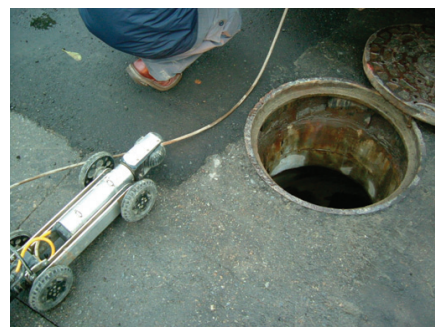

(a)

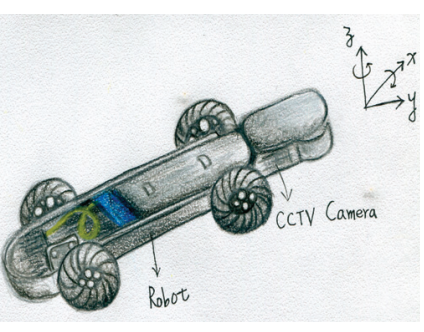

(b)

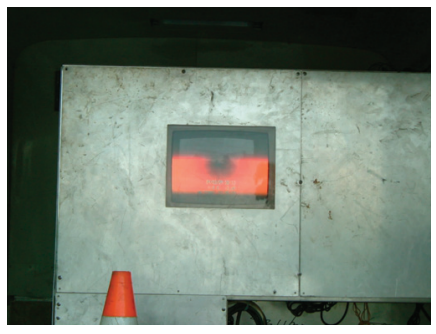

(c)

The inspection results show that cracks and open joints are two typical pipeline defects causing leakage problems in the sewer system. Due to the dark environment inside sewerage, a light beam from the CCTV robot is needed during CCTV inspection. The optical axis of CCTV robot would be usually parallel to the central axis of pipeline during sewer inspection. Consequently, open joints are apt to be illuminated by the CCTV robot because the surface of an open joint is usually perpendicular to the central axis of the pipeline. Cracks in CCTV images usually can be regarded as clearly dark patterns [14], but sometimes appear as bright patterns under several specific imaging conditions.

To demonstrate the performance of OTHO, CBHO, and MSED in the pipeline defect detection, 20 inspection images, in which each of cracks and open joints were individually recorded in 10 inspection images, were offered for training the three segmentation methods. The applicability of the proposed segmentation methods in the detection of cracks and open joints was also discussed. Then, other 40 inspection images recording either cracks or open joints were used for testing the proposed segmentation methods.

\subsection{Applicability Assessment of OTHO, CBHO and MSED in Pipeline Defect Detection}

Figures 3 and 4 show 10 inspection images of cracks and open joints, respectively, used for assessing the applicability of OTHO, CBHO, and MSED in pipeline defect detection. To reduce the computation, this research detected pipeline defects by probing the discontinuities in gray level instead of color imagery. The human interpretation of the pipeline defects in Figures 3 and 4 is shown in Figures 5 and 6, respectively. The white image regions express the interpreted pipeline defect, whereas the black ones represent the background environment. 
Figure 3. CCTV images of cracks.

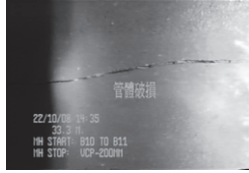

(a)

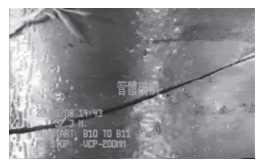

(f)

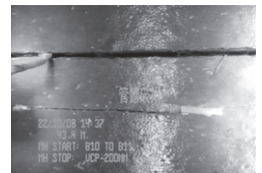

(b)

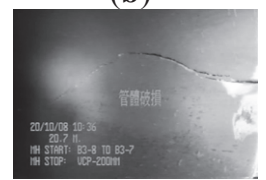

(g)

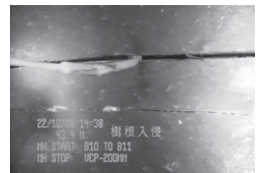

(c)

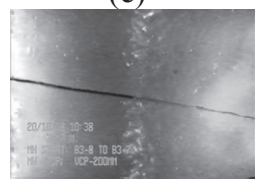

(h)

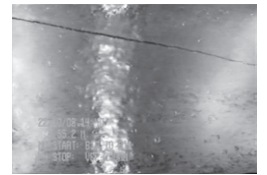

(d)

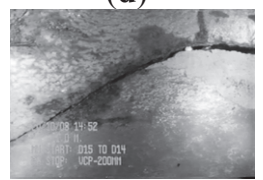

(i)

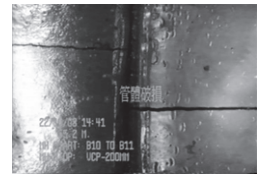

(e)

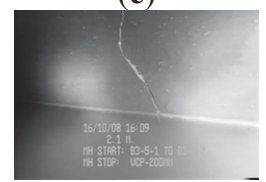

(j)

Figure 4. CCTV images of open joints.

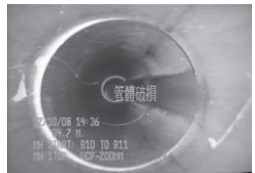

(a)

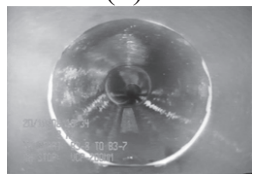

(f)

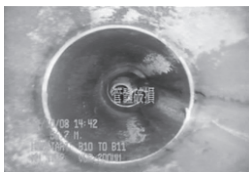

(b)

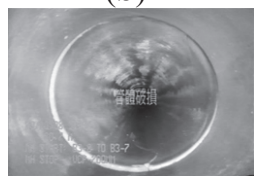

(g)

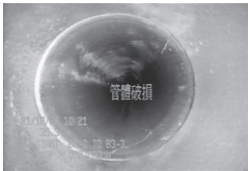

(c)

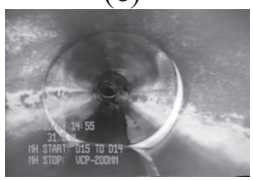

(h)

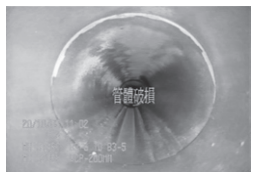

(d)

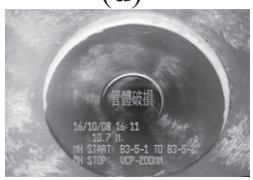

(i)

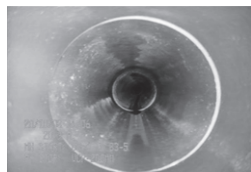

(e)

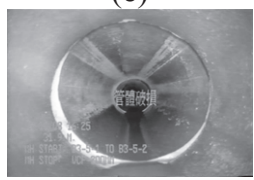

(j)

Figure 5. Manual interpretation of cracks corresponding to images in Figure 3.

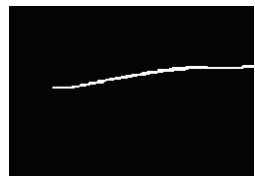

(a)

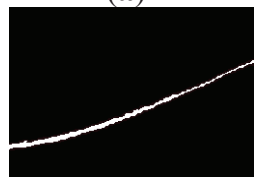

(f)

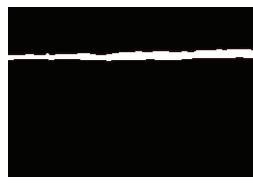

(b)

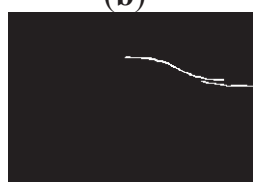

(g)

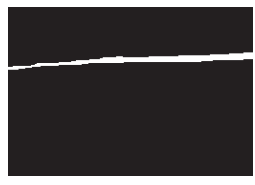

(c)

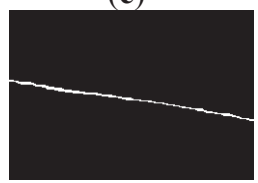

(h)

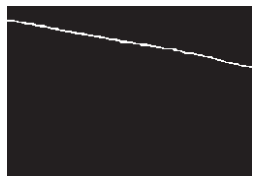

(d)

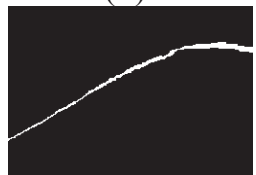

(i)

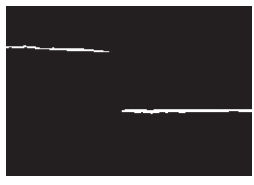

(e)

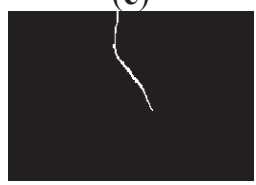

(j)

Figure 6. Manual interpretation of open joints corresponding to images in Figure 4.

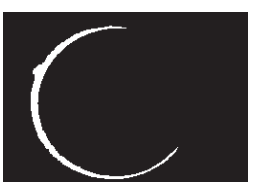

(a)

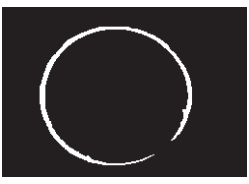

(b)

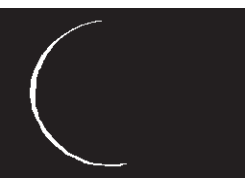

(c)

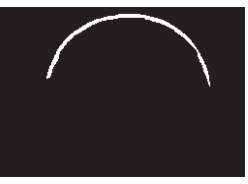

(d)

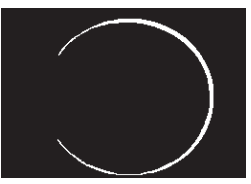

(e) 
Figure 6. Cont.

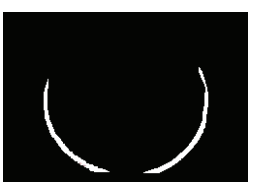

(f)

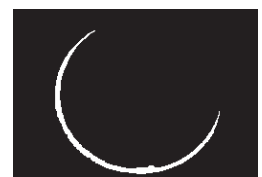

(g)

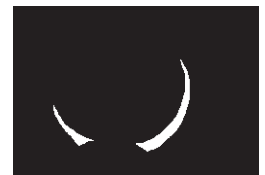

(h)

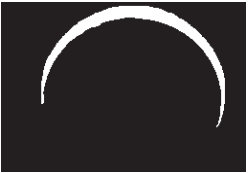

(i)

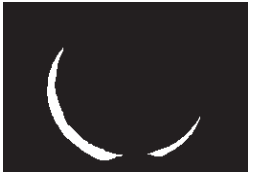

(j)

\subsubsection{Pipeline Defect Detection Using OTHO}

Figures 7 and 8 are the segmentation applying OTHO to the images in Figures 3 and 4, respectively. Based on Equation (9) through Equation (11), Table 1 lists the segmentation accuracy in Figures 7 and 8, respectively, and shows that OTHO is better in open joint detection than crack detection. In Figure 4, open joints look like ring patterns with a strong illumination, whereas most cracks present slender gap patterns without illumination in Figure 3. Figures 7 and 8 show that pipeline defects or noisy environments with stronger illumination than their neighbors were detected by OTHO as white image regions.

Figure 7. Segmentation of crack CCTV images by OTHO.

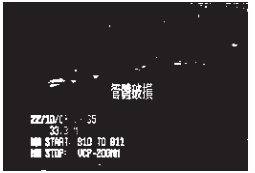

(a)

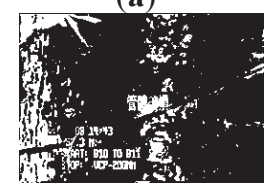

(f)

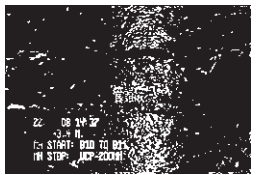

(b)

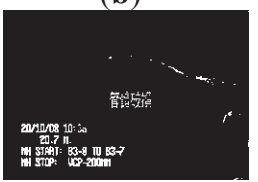

(g)

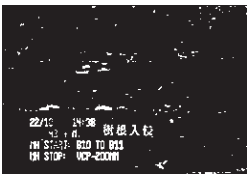

(c)

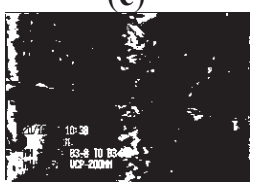

(h)

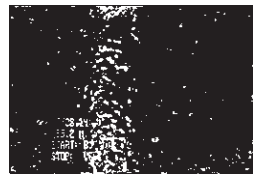

(d)

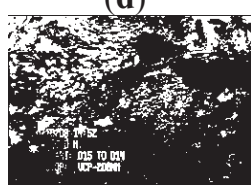

(i)

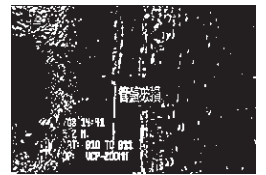

(e)

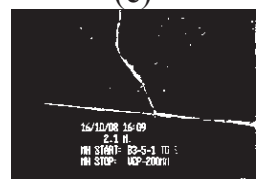

(j)

Figure 8. Segmentation of open joint CCTV images by OTHO.

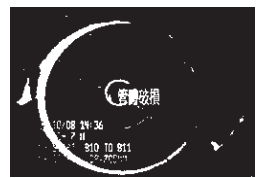

(a)

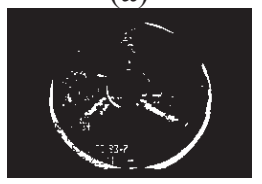

(f)

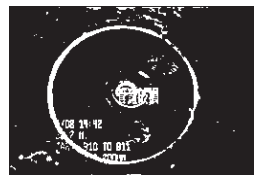

(b)

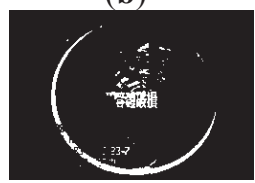

(g)

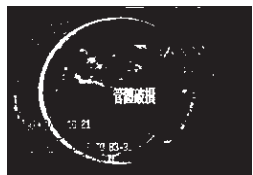

(c)

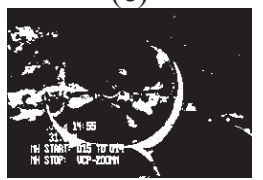

(h)

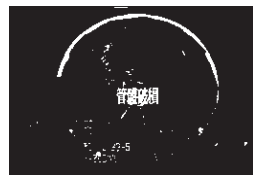

(d)

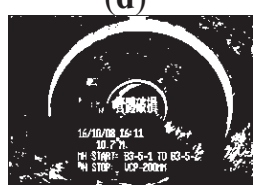

(i)

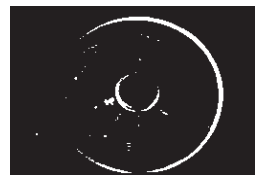

(e)

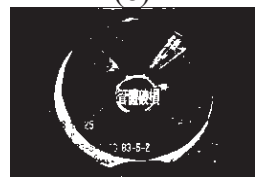

(j) 
Table 1. Segmentation accuracy of crack (C) and open joint (OJ) segmentations by OTHO.

\begin{tabular}{ccccccc}
\hline \multirow{2}{*}{ Image ID } & \multicolumn{7}{c}{ Accuracy Index (\%) } \\
\cline { 2 - 7 } & \multicolumn{2}{c}{ Compl } & \multicolumn{3}{c}{ Corr } & \multicolumn{2}{c}{ Quality } \\
\cline { 2 - 7 } (a) & 8.28 & 98.26 & 2.80 & 49.25 & 2.14 & 48.82 \\
(b) & 6.91 & 88.14 & 3.39 & 41.12 & 2.33 & 38.97 \\
(c) & 2.44 & 83.92 & 1.60 & 31.32 & 0.97 & 29.55 \\
(d) & 1.56 & 91.73 & 0.28 & 47.33 & 0.23 & 45.40 \\
(e) & 1.41 & 88.16 & 0.19 & 74.39 & 0.17 & 67.64 \\
(f) & 19.93 & 86.21 & 4.77 & 55.92 & 4.00 & 51.33 \\
(g) & 5.40 & 87.43 & 0.91 & 56.24 & 0.79 & 52.03 \\
(h) & 0.56 & 92.66 & 0.07 & 19.21 & 0.07 & 18.93 \\
(i) & 9.57 & 92.56 & 0.78 & 39.66 & 0.73 & 38.44 \\
(j) & 71.60 & 91.30 & 8.64 & 47.97 & 8.35 & 45.88 \\
Average & 6.23 & 89.90 & 1.64 & 46.05 & 1.27 & 43.46 \\
Standard Deviation & 6.08 & 4.32 & 1.65 & 15.89 & 1.31 & 14.11 \\
\hline
\end{tabular}

p.s. The extreme values of Image (j) of crack are excluded in calculation of average and standard deviation.

In Table 1, OTHO offering an averaged Compl of $89.90 \%$ coupled with a standard deviation of $4.32 \%$ for open joint detection, indicating that most image regions belonging to open joints can be well detected. However, not only the open joints but also the inspection texts or the environmental noise were detected so much the Corr is far inferior to the Compl. The inspection texts, including date, manholes of departure and arrival, pipe material, diameters, and category of pipe defect, were recoded and placed on the inspection image. If the tone of the inspection texts against their neighbor background is clear, the inspection texts have a high probability of being segmented and decrease the Corr. Thus, in the future a separate attribute database to record the inspection texts is necessary to avoid incorrect detections of pipeline defects. In addition to the inspection texts, we found that the heterogeneous illumination due to the reflection by the noisy environments would also result in the incorrect detection of pipeline defects and worsened Quality.

Table 1 also shows that applying OTHO to the open joint detection in Figure 4e obtained the best Quality of $67.64 \%$, where Compl and Corr are $88.16 \%$ and $74.39 \%$, respectively. Figure 8e shows that OTHO has the capability of detecting the near open joint (larger white ring) and the far open joint (smaller white ring) from the CCTV position. However, the near open joint referred to in Figure 6e should be the only target of concern under the assumption of only one defect existing in one image in this research. The Corr of $74.39 \%$ can be improved by ignoring the far open joint.

As for the crack detection by OTHO, the best Compl of $71.60 \%$ was obtained for the crack in Figure $3 \mathrm{j}$ due to its strong illumination. However, the transverse stripe of non-crack and the inspection texts in Figure $3 \mathrm{j}$ were also detected so that the Corr of merely $8.64 \%$ indicates that OTHO is unsuitable for crack detection. 


\subsubsection{Pipeline Defect Detection Using $\mathrm{CBHO}$}

The segmentation applying CBHO to the corresponding to images in Figures 3 and 4 are shown in Figures 9 and 10, respectively. The accuracy of the detected pipeline defects in Figures 9 and 10 is estimated in Table 2. Obviously, CBHO derives better performance in detecting cracks than open joints, contrary to OTHO. Comparing Tables 1 and 2, Quality of crack detection is significantly improved and demonstrates that $\mathrm{CBHO}$ is suitable for detecting objects which absorb light beams. Excluding Figure 9a and 9j, most segmented cracks in Figure 9 have Compl above 50\% by CBHO. In particular, the crack in Figure 9e obtained a Compl of $82.79 \%$. Also, the inspection text or environmental noise was detected by $\mathrm{CBHO}$ so that the crack in Figure 9e has Corr of $8.94 \%$ which is far inferior to Compl of $82.79 \%$. Consequently, the low Corr results in low Quality of $8.78 \%$.

Figure 9. Segmentation of crack CCTV images by CBHO.

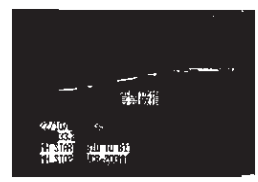

(a)

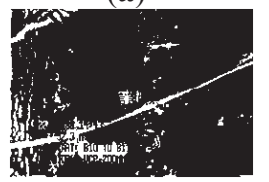

(f)

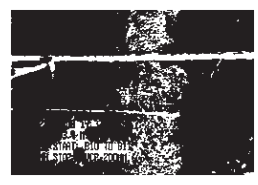

(b)

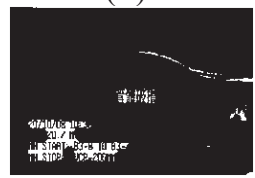

(g)

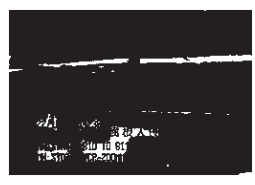

(c)

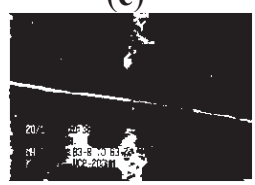

(h)

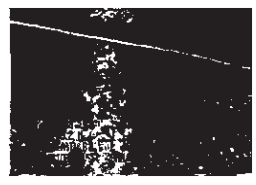

(d)

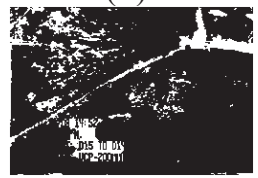

(i)

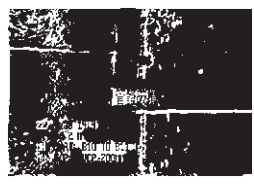

(e)

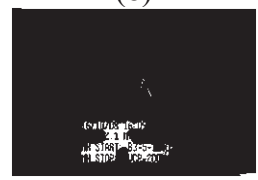

(j)

Figure 10. Segmentation of open joint CCTV images by CBHO.

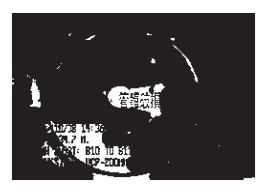

(a)

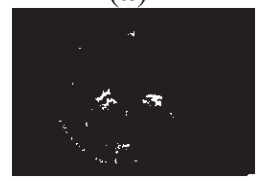

(f)

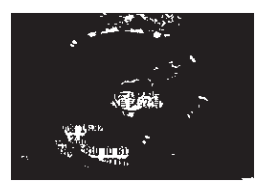

(b)

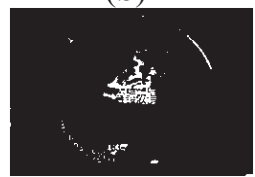

(g)

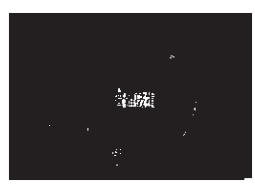

(c)

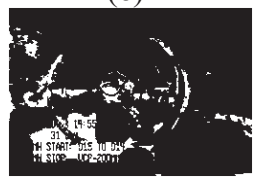

(h)

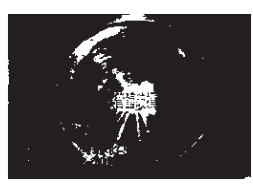

(d)

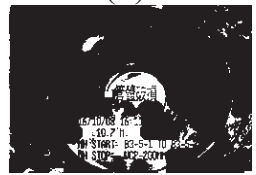

(i)

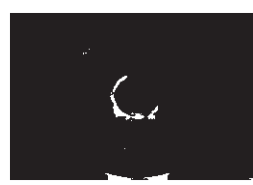

(e)

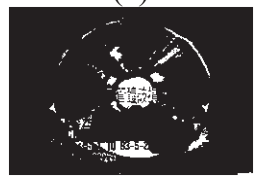

(j)

Table 2 also shows that $\mathrm{CBHO}$ is unsuitable for open joint detection due to low Quality. Even, image (e) was given the Quality of 0\% with Compl and Corr both being 0\%. Figure 10e is seen that the farther open joint in Figure $4 \mathrm{e}$ was segmented as the ring-like image region, but the farther open joint is not the detected defect shown as Figure 6e. In addition to the ring-like segmentation, the other segmented image region is located at the bottom of Figure 10e. Unfortunately, the segmented image regions in Figure 10e do not match the manual interpretation in Figure 6e at all. The above result illustrates that $\mathrm{CBHO}$ would lead to pseudosegmentation for open joints. 
Table 2. Segmentation accuracy of crack (C) and open joint (OJ) segmentations by CBHO.

\begin{tabular}{ccccccc}
\hline \multirow{2}{*}{ Image ID } & \multicolumn{7}{c}{ Accuracy Index (\%) } \\
\cline { 2 - 7 } & \multicolumn{2}{c}{ Compl } & Corr & \multicolumn{2}{c}{ Quality } \\
\cline { 2 - 7 } (a) & 27.48 & 6.89 & 9.56 & 3.14 & 7.63 & OJ \\
\hline (b) & 56.89 & 0.51 & 21.89 & 0.43 & 18.77 & 0.23 \\
(c) & 71.39 & 0.12 & 39.35 & 0.18 & 33.99 & 0.07 \\
(d) & 70.18 & 0.11 & 11.51 & 0.03 & 10.97 & 0.03 \\
(e) & 82.79 & 0.00 & 8.94 & 0.00 & 8.78 & 0.00 \\
(f) & 53.43 & 2.65 & 22.21 & 4.58 & 18.61 & 1.71 \\
(g) & 52.16 & 2.35 & 6.21 & 4.46 & 5.87 & 1.57 \\
(h) & 65.86 & 2.25 & 9.75 & 2.30 & 9.28 & 1.15 \\
(i) & 79.65 & 10.66 & 11.72 & 2.45 & 11.37 & 2.03 \\
(j) & 4.94 & 1.16 & 0.73 & 0.36 & 0.64 & 0.28 \\
Average & 62.20 & 2.67 & 15.68 & 1.79 & 13.92 & 0.93 \\
Standard Deviation & 16.96 & 3.48 & 10.50 & 1.83 & 8.77 & 0.90 \\
\hline
\end{tabular}

p.s. The extreme values of Image (j) of crack are excluded in calculation of average and standard deviation.

\subsubsection{Pipeline Defect Detection Using MSED}

Figures 11 and 12 show the segmentation applying MSED to Figures 3 and 4, respectively. Table 3 presents the segmentation accuracy for Figures 11 and 12, and shows that MSED offers better performance for open joint detection than crack detection. Moreover, a comparison between Tables 2 and 3 shows MSED is superior to CBHO in crack detection due to the less segmentation of environmental noise. However, the inspection texts were also detected by MSED as white rectangles (see Figures 11 and 12), and this greatly deteriorated the segmentation. In Table 3, the best Quality of $26.51 \%$ with Compl of $80.29 \%$ and Corr of $28.36 \%$ was obtained for the crack detection in Figure 3c. Especially, the Corr of $28.36 \%$ can be greatly improved if the inspection texts were removed from the image. Similarly, in Table 3 lower Corr values, such as $7.60 \%, 5.11 \%$ or $5.12 \%$, resulted from the detection of the inspection texts so as to deteriorate the Quality of $6.91 \%, 5.01 \%$ or $5.03 \%$ (see Figure $11 \mathrm{a}$, g or j). Although a crack usually appears as a slender gap pattern without any illumination, comparison of Table 3 to Table 2 demonstrates that MSED has better capability of crack detection than $\mathrm{CBHO}$, unless a crack is located in shadows or is smoothed by the surroundings. Noticeably, the pattern of a crack in Figure $3 \mathrm{~b}$ is extremely visible. However, a Compl of merely $49.51 \%$ was obtained by MSED for the crack detection that is caused by the parameter, dlim, given by a length of 7 pixels, which is short for the MSED algorithm in complete crack detection (see Figure 11b). 
Figure 11. Segmentation of crack CCTV images by MSED.

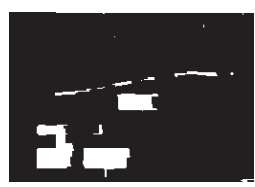

(a)

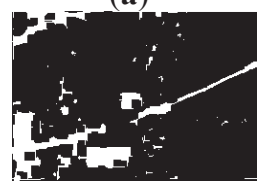

(f)

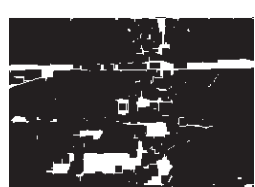

(b)

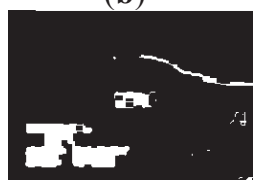

(g)

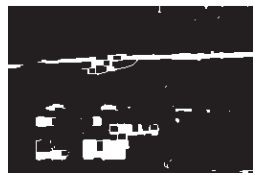

(c)

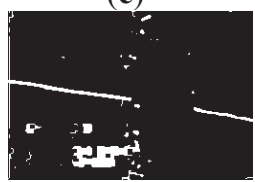

(h)

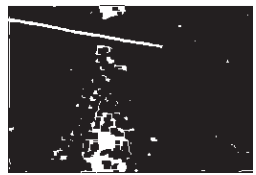

(d)

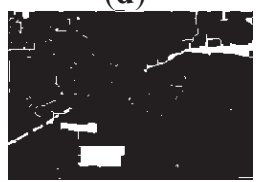

(i)

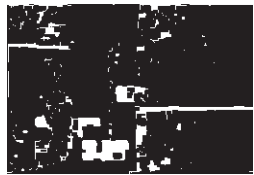

(e)

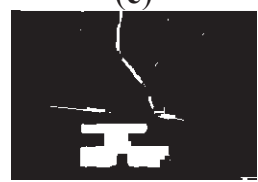

(j)

Figure 12. Segmentation of open joint CCTV images by MSED.

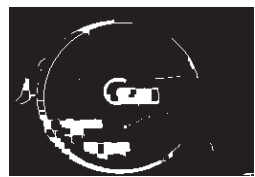

(a)

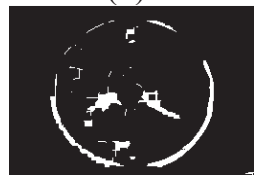

(f)

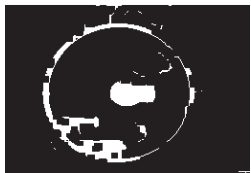

(b)

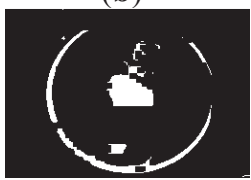

(g)

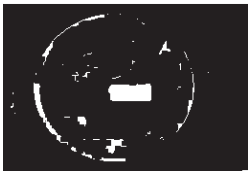

(c)

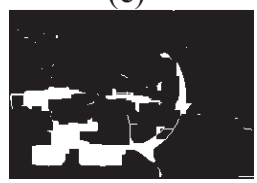

(h)

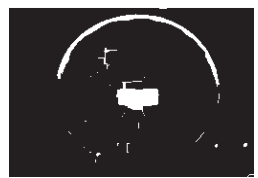

(d)

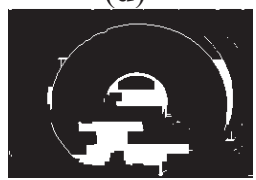

(i)

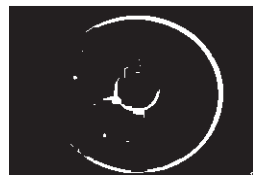

(e)

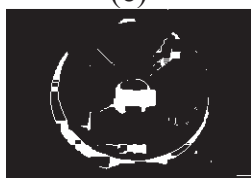

(j)

Table 3. Segmentation accuracy of crack (C) and open joint (OJ) segmentations by MSED.

\begin{tabular}{|c|c|c|c|c|c|c|}
\hline \multirow{3}{*}{ Image ID } & \multicolumn{6}{|c|}{ Accuracy Index (\%) } \\
\hline & \multicolumn{2}{|c|}{ Compl } & \multicolumn{2}{|c|}{ Corr } & \multicolumn{2}{|c|}{ Quality } \\
\hline & $\mathbf{C}$ & OJ & C & OJ & C & OJ \\
\hline (a) & 43.21 & 50.17 & 7.60 & 24.70 & 6.91 & 19.84 \\
\hline (b) & 49.51 & 64.02 & 25.47 & 30.96 & 20.22 & 26.37 \\
\hline (c) & 80.29 & 58.22 & 28.36 & 22.44 & 26.51 & 19.33 \\
\hline (d) & 67.45 & 97.70 & 12.57 & 37.31 & 11.85 & 36.98 \\
\hline (e) & 76.53 & 90.89 & 12.23 & 57.62 & 11.79 & 54.47 \\
\hline (f) & 62.14 & 87.38 & 22.59 & 38.87 & 19.86 & 36.80 \\
\hline (g) & 72.30 & 88.87 & 5.11 & 37.47 & 5.01 & 35.79 \\
\hline (h) & 83.30 & 71.50 & 17.87 & 15.58 & 17.25 & 14.67 \\
\hline (i) & 58.20 & 20.03 & 18.17 & 10.63 & 16.07 & 7.46 \\
\hline (j) & 73.66 & 62.77 & 5.12 & 33.74 & 5.03 & 28.11 \\
\hline Average & 66.66 & 69.16 & 15.51 & 30.93 & 14.05 & 27.98 \\
\hline Standard Deviation & 13.24 & 23.51 & 8.33 & 13.44 & 7.19 & 13.60 \\
\hline
\end{tabular}


In Table 3, the four segmented images of open joints, including images (d), (e), (f), and (g), gave better Quality. Especially for image (e), Quality of 54.47\% with Compl of $90.89 \%$ and Corr of $57.62 \%$ was the optimum derived by MSED. As in Figures 8e and 10e, in Figure 12e the farther open joint was also detected by MSED as a smaller white ring that decreased segmentation correctness. In other words, the Corr of $57.62 \%$ is an underestimation for MSED in the performance of correctly detecting pipeline defects. In Table 3, the best and worst Compls were obtained for the segmentation of Figure 12d and 12i, respectively. The open joints in Figure 4d and $4 \mathrm{i}$ have similar patterns. The greatest difference between two patterns is the illuminated areas of the open joints due to the large displacement of the open joint in Figure 4i compared with Figure 4d. Unfortunately, the upper edge of the open joint in Figure $4 i$ failed to be detected by edge detection causing the MSED algorithm to be ineffective in the open joint detection (see Figure 12i). The failed edge detection was caused the image gradients below the threshold which was automatically determined by Otsu's technique.

\subsubsection{Summary of Pipeline Defect Detection Using OTHO, CBHO and MSED}

On a $2.5 \mathrm{GHz}$ PC with four CPUs, less than $0.1,0.5$, and 1 second was taken to process the MATLAB code of CBHO, OTHO, and MSED, respectively. With superior computation efficiency, in-time pipeline defect detection can be expected. Based on the illustration of applying OTHO, $\mathrm{CBHO}$, and MSED to the detection of crack and open joint, a summary of the pipeline defect detection using OTHO, CBHO, and MSED is obtained and discussed as follows:

(1) Tables 1 and 3 show that Quality of open joint detection is higher than that of crack detection. Cracks were found to be more difficult to detect due to their varied appearance. The Quality obtained by MSED (see Table 3) is significantly better than that by OTHO for the crack detection (see Table 1), whereas OTHO is superior to MSED in open joint detection. The comparison in Tables 2 through 3 indicates that MSED seems better than $\mathrm{CBHO}$ in both crack and open joint detection. Hence, this paper suggests that OTHO and MSED can be considered suitable for open joint and crack detections, respectively.

(2) The difficulty to derive a high Corr results from the serious interference from the noisy environment which is unavoidable during sewer inspection. OTHO, offering Compl between $83.92 \%$ and $98.26 \%$ for the open joint detection (see Table 1), is proven useful for open joint detection. Compl values up to $83.30 \%$ and above $60 \%$ for seven of the 10 inspection images derived by MSED for the crack detection (see Table 3) demonstrate the effectiveness of MSED in crack detection.

(3) Figures 11 and 12 show that most inspection texts were detected by MSED as image regions consisting of white rectangles. However, the sizes of the white rectangles are mostly larger than those of the detected inspection texts by OTHO. The larger the detected inspection texts are, the more inferior the Corr is. In addition, the number of the image regions detected by MSED is significantly less than that by OTHO. In Figures 7 and 8, many small image regions due to the noisy environment, such as heterogeneous illumination, were detected by OTHO and this severely deteriorated Corr. 
(4) Figures 7 through 12 show that in crack detection the three segmentation methods suffer from the deterioration caused by environmental noise. The segmentation of environmental noise results from the corroded pipe wall which reflects heterogeneous beams back to the CCTV camera. In an image with heterogeneous beam reflection, OTHO and CBHO would segment brighter and darker portions, respectively. MSED would detect the edges between the bright and dark portions in image segmentation. The segmented environmental noise frequently has the morphological feature of fractals which is extremely different from the line-like features of cracks or the ring-like features of open joints. In future work, criteria based on morphological features should be established to remove the environmental noise, and the effect of the noise removal will be discussed.

\subsection{Verification of OTHO and MSED in Pipeline Defect Detection}

To verify the applicability of OTHO and MSED in open joint and crack detection, this research employed additional 40 inspection images for each of crack and open joint. Based on the total 80 inspection images, Figure 13 shows Compl, Corr, and Quality for the applicability verification. Figure 13 displays the accuracies of MSED and OTHO tests in the crack and open joint detections, respectively. The long bar represents a great difference between the maximum and minimum of Compl. Averaged Compl values of $61.50 \%$ and $86.72 \%$ were obtained for the crack and open joint detections, respectively. The standard deviations of Compl in the crack and open joint detections are $19.98 \%$ and $13.59 \%$, respectively. In conclusion, OTHO has a robust capability of detecting open joints and MSED can effectively detect cracks.

Figure 13. Verification of pipeline defect detection. (a) Crack detection using MSED. (b) Open joint detection using OTHO.

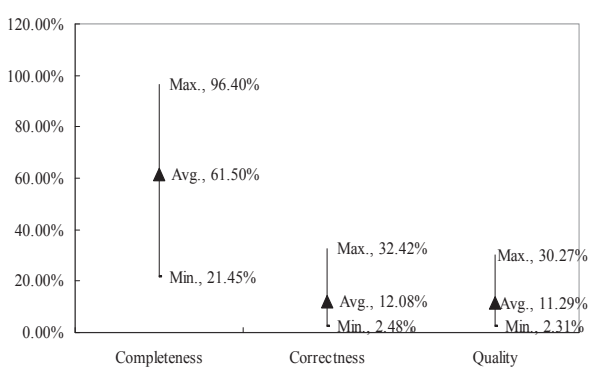

(a)

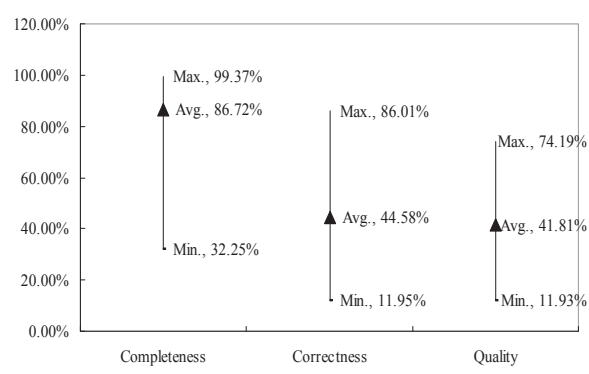

(b)

Figure 13 also shows Corr of the open joint detection ranging significantly wider than that of the crack detection. The standard deviations of Corr for the open joint and crack detections are $18.49 \%$ and $5.89 \%$, respectively, which is similar to that of Quality. Compared with Figure 13a and $13 \mathrm{~b}$, Corr is greatly inferior to Compl due to the influence of the noisy environment. Moreover, it is more difficult to correctly detect cracks than open joints so that Quality of the crack detection is relatively lower than that of the open joint detection. Table 4 is a statistical analysis of the different levels of the accuracy indices for the pipeline defect detection. For the crack detection by MSED, 
almost all 40 inspected images obtaining both Corr and Quality less than 20\% indicates that the noisy environment has a great impact on MSED performance of pipeline defect detection. For the open joint detection using OTHO, most of 40 inspected images obtained Compl values above $80 \%$ and Corr ones between 40\% and 80\%. Moreover, Corr has greater impact on Quality than Compl.

Table 4. Inspected image number in different levels of accuracy indices for pipeline defect detection.

\begin{tabular}{|cc|ccccc|}
\hline Index & Percentage (\%) & $\mathbf{2 0}$ & $\mathbf{4 0}>\sim \geq \mathbf{2 0}$ & $\mathbf{6 0}>\sim$ 40 & $\mathbf{8 0}>\sim$ 60 & $\geqq \mathbf{8 0}$ \\
\hline \multirow{4}{*}{ Crack detection using MSED } & Compl & 0 & 5 & 12 & 16 & 7 \\
& Corr & 38 & 2 & 0 & 0 & 0 \\
& Quality & 39 & 1 & 0 & 0 & 0 \\
\hline \multirow{2}{*}{ Open joint detection using } & Compl & 0 & 1 & 1 & 5 & 33 \\
OTHO & Corr & 6 & 8 & 17 & 8 & 1 \\
& Quality & 6 & 15 & 11 & 8 & 0 \\
\hline
\end{tabular}

\section{Conclusions}

This paper proposes a novel image segmentation method, morphological segmentation based on edge detection (MSED), to detect the pipeline defects, including cracks and open joints, which are the typical pipeline defects causing leaking problems in vitrified clay sewage pipelines. The developed image segmentation methods, opening top-hat operation (OTHO) and closing bottom-hat operation ( $\mathrm{CBHO})$, were also applied to the pipeline defect detection for comparison. Various indices, including completeness, correctness, and quality, were introduced into the accuracy assessment of the pipeline defect detection. Cracks were found to be more difficult to detect due to their varied appearance. The test results demonstrate that MSED outperforms both $\mathrm{CBHO}$ and OTHO in crack detection, and OTHO outperforms both CBHO and MSED in open joint detection. In conclusion, this paper suggests employing MSED for cracks and OTHO for open joints in detection processing.

The sewer pipes in the 9th district sewer system of Taichung City were made of vitrified clay, which is a typical rigid pipe material. The pipeline defect detection methods proposed in this research can also be applied to other rigid pipes, such as concrete pipes. However, the inspection texts recorded onto the acquired CCTV images deteriorate the correctness and quality. The barrier of the noisy environment segmentation for MSED or OTHO remains. We strongly suggest that a separate attribute database for inspection texts is necessary in future CCTV shooting processes. Removal of the inspection texts from CCTV images should significantly improve the detection correctness. Moreover, some morphology-based decision criteria or fitness function [37] could be established in the future for removal of the noisy environment to improve the correctness and quality of detection. According to the comment by Kirstein et al. [29], the irregular environment inside sewer pipelines results in a long time still being needed for developing an automated sewer inspection system. In further study, ground light detection and ranging (LiDAR) can be introduced and coupled with a synchronous camera for pipeline defect measurement based on point clouds 
with precise coordinates. Nevertheless, this paper demonstrates that the different types of pipeline defects need different image segmentation methods for detection. Thus, identifying an appropriate image segmentation method for the detection of objective pipeline defects also needs advanced study.

\section{Author Contributions}

Tung-Ching Su conceived the idea for the project and was principal to all phases of the research, including the inspection images acquiring, the MSED algorithm encoding, result analysis, comparison of MSED with the other image segmentation methods, literature review, and manuscript preparation. Ming-Der Yang contributed to the research activity supervising, the interpretation of the modeling results, and manuscript wording and adaptation.

\section{Conflicts of Interest}

The authors declare no conflict of interest.

\section{References}

1. Construction and Planning Agency, Ministry of the Interior Website. Available online: http://www.cpami.gov.tw/chinese/index.php?option=com_content\&view=article\&id=9995\&It emid=53 (accessed on 16 April 2013).

2. Nikles, M.; Vogel, B.; Briffod, F.; Grosswig, S.; Sauser, F.; Luebbecke, S.; Bals, A.; Pfeiffer, T. Leakage Detection Using Fiber Optics Distributed Temperature Monitoring. Proc. SPIE 2004, doi:10.1117/12.540270.

3. Inaudi, D.; Glisic, B. Distributed Fiber-Optic Sensing for Long-Range Monitoring of Pipelines. In Proceedings of the 3rd International Conference on Structural Health Monitoring of Intelligent Infrastructure, Vancouver, British Columbia, Canada, 13-16 November 2007.

4. Nikles, M. Long-Distance Fiber Optic Sensing Solutions for Pipeline Leakage, Intrusion and Ground Movement Detection. Proc. SPIE 2009, doi:10.1117/12.818021.

5. Inaudi, D.; Glisic, B. Long-range pipeline monitoring by distributed fiber optic sensing. J. Press. Vessel Tech. 2010, doi: 10.1115/1.3062942.

6. Yang, M.D.; Su, T.C. Automation model of sewerage rehabilitation planning. Water Sci. Tech. 2006, 54, 225-232.

7. Yang, M.D.; Su, T.C. An optimization model of sewerage rehabilitation. J. Chin. Inst. Eng. 2007, 30, 651-659.

8. Yang, M.D.; Su, T.C.; Pan, N.F.; Liu, P. Feature extraction of sewer pipe defects using wavelet transform and co-occurrence matrix. Int. J. Wavelets Multiresolut. Inf. Process. 2011, 9, 211-225.

9. Yang, M.D.; Su, T.C.; Pan, N.F.; Yang, Y.F. Systematic image quality assessment for sewer inspection. Expert Syst. Appl. 2011, 38, 1766-1776.

10. Su, T.C.; Yang, M.D.; Wu, T.C.; Lin, J.Y. Morphological segmentation based on edge detection for sewer pipe defects on CCTV images. Expert Syst. Appl. 2011, 38, 13094-13114. 
11. Makar, J.M. Diagnostic techniques for sewer systems. J. Infrastruct. Syst. 1999, 5, 69-78.

12. Fenner, R.A. Approaches to sewer maintenance: a review. Urban Water 2000, 2, 343-356.

13. Gokhale, S.; Graham, J.A. A new development in locating leaks in sanitary sewers. Tunn. Undergr. Sp. Tech. 2004, 19, 85-96.

14. Yang, M.D.; Su, T.C. Automated diagnosis of sewer pipe defects based on machine learning approaches. Expert Syst. Appl. 2008, 35, 1327-1337.

15. Wirahadikusumah, R.; Abraham, D.M.; Iseley, T.; Prasanth, R.K. Assessment technologies for sewer system rehabilitation. Automat. Constr. 1998, 7, 259-270.

16. Mckim, R.A.; Sinha, S.K. Condition assessment of underground sewer pipes using a modified digital image processing paradigm. Tunn. Undergr. Sp. Tech. 1999, 14, 29-37.

17. Moselhi, O.; Shehab-Eldeen, T. Classification of defects in sewer pipes using neural network. J. Infrastruct. Syst. 2000, 6, 97-104.

18. Iyer, S.; Sinha, S.K. A robust approach for automatic detection and segmentation of cracks in underground pipeline images. Image Vision Comput. 2005, 23, 921-933.

19. Sinha, S.K.; Fieguth, P.W. Segmentation of buried concrete pipe images. Automat. Constr. 2006, 15, 47-57.

20. Yang, M.D.; Su, T.C. Segmenting ideal morphologies of sewer pipe defects on CCTV images for automated diagnosis. Expert Syst. Appl. 2009, 36, 3562-3573.

21. Zana, F.; Klein, J.C. Segmentation of vessel-like patterns using mathematical morphology and curvature evaluation. IEEE Trans. Image Process. 2001, 10, 1010-1019.

22. Shehab, T.; Moselhi, O. Automated detection and classification of infiltration in sewer pipes. J. Infrastruct. Syst. 2005, 11, 165-171.

23. Lee, J.H.; Yoo, H.S.; Kim, Y.S.; Lee, J.B.; Cho, M.Y. The development of a machine vision-assisted, teleoperated pavement crack sealer. Automat. Constr. 2006, 15, 616-626.

24. Li, Y.; Ai, J.; Sun, C. Online fabric defect inspection using smart visual sensors. Sensors 2013, 13, 4659-4673.

25. Lin, Q.; Miao, W.; Zhang, W.; Fu, Q.; Wu, N.A. 1000 frames/s programmable vision chip with variable resolution and row-pixel-mixed parallel image processors. Sensors 2009, 9, 5933-5951.

26. Hsiao, Y.T.; Chuang, C.L.; Lu, Y.L.; Jiang, J.A. Robust multiple objects tracking using image segmentation and trajectory estimation scheme in video frames. Image Vision Comput. 2006, 24, 1123-1136.

27. Chen, T.; Wu, Q.H.; Rahmani-Torkaman, R.; Hughes, J. A pseudo top-hat mathematical morphological approach to edge detection in dark regions. Pattern Recognit. 2002, 35, 199-210.

28. Jin, L.; Xian, H.; Bie, J.; Sun, Y.; Hou, H.; Niu, Q. License plate recognition algorithm for passenger cars in Chinese residential areas. Sensors 2012, 12, 8355-8370.

29. Kirstein, S.; Müller, K.; Walecki-Mingers, M.; Deserno, T.M. Robust adaptive flow line detection in sewer pipes. Autom. Constr. 2012, 21, 24-31.

30. Shih, F.Y. Image Processing and Pattern Recognition; John Wiley \& Sons: Hoboken, NJ, USA, 2010; pp. 52-55. 
31. Lin, C.L. An approach to improve the quality of infrared images of vein-patterns. Sensors 2011, 11, 11447-11463.

32. Aguzzi, J.; Costa, C.; Robert, K.; Matabos, M.; Antonucci, F.; Juniper, S.K.; Menesatti, P. Automated image analysis for the detection of benthic crustaceans and bacterial mat coverage using the VENUS undersea cabled network. Sensors 2011, 11, 10534-10556.

33. Yang, R.; Gabbouj, M.; Neuvo, Y. Fast algorithms for analyzing and designing weighted median filters. Signal Process. 1995, 41, 135-152.

34. Dong, P. Implementation of mathematical morphological operations for spatial data processing. Comput. Geosci. 1997, 23, 103-107.

35. Li, T.G.; Wang, S.P.; Zhao, N. Gray-scale edge detection for gastric tumor pathologic cell images by morphological analysis. Comput. Biol. Med. 2009, 39, 947-952.

36. Gonzalez, R.C.; Woods, R.E. Digital Image Processing, 2nd ed.; Prentice Hall: New York, NY, USA, 2002.

37. Yang, M.D.; Yang, Y.F.; Su, T.C.; Huang, K.S. An efficient fitness function in genetic algorithm classifier for landuse recognition on satellite images. The Scientific World J. 2014, doi:10.1155/2014/264512. 


\title{
Implementation of Ultrasonic Sensing for High Resolution Measurement of Binary Gas Mixture Fractions
}

\author{
Richard Bates, Michele Battistin, Stephane Berry, Alexander Bitadze, Pierre Bonneau, \\ Nicolas Bousson, George Boyd, Gennaro Bozza, Olivier Crespo-Lopez, Enrico Da Riva, \\ Cyril Degeorge, Cecile Deterre, Beniamino DiGirolamo, Martin Doubek, Gilles Favre, \\ Jan Godlewski, Gregory Hallewell, Ahmed Hasib, Sergey Katunin, Nicolas Langevin, \\ Didier Lombard, Michel Mathieu, Stephen McMahon, Koichi Nagai, Benjamin Pearson, \\ David Robinson, Cecilia Rossi, Alexandre Rozanov, Michael Strauss, Michal Vitek, \\ Vaclav Vacek and Lukasz Zwalinski
}

\begin{abstract}
We describe an ultrasonic instrument for continuous real-time analysis of the fractional mixture of a binary gas system. The instrument is particularly well suited to measurement of leaks of a high molecular weight gas into a system that is nominally composed of a single gas. Sensitivity $<5 \times 10^{-5}$ is demonstrated to leaks of octaflouropropane $\left(\mathrm{C}_{3} \mathrm{~F}_{8}\right)$ coolant into nitrogen during a long duration (18 month) continuous study. The sensitivity of the described measurement system is shown to depend on the difference in molecular masses of the two gases in the mixture. The impact of temperature and pressure variances on the accuracy of the measurement is analysed. Practical considerations for the implementation and deployment of long term, in situ ultrasonic leak detection systems are also described. Although development of the described systems was motivated by the requirements of an evaporative fluorocarbon cooling system, the instrument is applicable to the detection of leaks of many other gases and to processes requiring continuous knowledge of particular binary gas mixture fractions.
\end{abstract}

Reprinted from Sensors. Cite as: Bates, R.; Battistin, M.; Berry, S.; Bitadze, A.; Bonneau, P.; Bousson, N.; Boyd, G.; Bozza, G.; Crespo-Lopez, O.; Da Riva, E.; Degeorge, C.; Deterre, C.; DiGirolamo, B.; Doubek, M.; Favre, G.; Godlewski, J.; Hallewell, G.; Hasib, A.; Katunin, S.; Langevin, N.; Lombard, D.; Mathieu, M.; McMahon, S.; Nagai, K.; Pearson, B.; Robinson, D.; Rossi, C.; Rozanov, A.; Strauss, M.; Vitek, M.; Vacek, V.; Zwalinski, L. Implementation of Ultrasonic Sensing for High Resolution Measurement of Binary Gas Mixture Fractions. Sensors 2014, 14, 11260-11276.

\section{Introduction}

Fractional measurement of binary gas mixtures with ultrasonic pulses has been in use for some decades in High Energy Physics (HEP) experiments; see for example [1]. These experiments use binary gas mixtures that must be accurately monitored and controlled in order to optimize the experimental data. Monitoring in these experiments is achieved by gas mixture analyzers operating on the principle that the speed of sound in binary gas mixtures of differing molecular masses is sensitive to the ratio of the molar fraction of the mixture (Figure 1). 
Recently, we have expanded the application of ultrasonic gas analysis to the silicon tracker of the ATLAS experiment (A Toroidal LHC ApparatuS) at the CERN Large Hadron Collider (LHC) for in situ leak detection [2].

The silicon detectors of the ATLAS Inner Detector are subdivided into the ATLAS Pixel Detector, using pixelated silicon sensors [3] and the SCT Detector (Semi-Conductor Tracker), constructed of silicon micro-strip sensors. They share a common evaporative cooling system using that uses the coolant octafluoropropane $\left(\mathrm{C}_{3} \mathrm{~F}_{8}\right)$, a saturated $\left(\mathrm{C}_{n} \mathrm{~F}_{(2 n+2)}\right)$ fluorocarbon with convenient thermodynamics. $\mathrm{C}_{3} \mathrm{~F}_{8}$ also features radiation resistance, non-toxicity, non-flammability and non-conductivity [4]. The coolant is delivered via capillaries (which cannot be allowed to clog) in 204 independently controlled cooling loops and provides local cooling to an average of approximately $-7{ }^{\circ} \mathrm{C}$ for the silicon sensors and their associated electronics, with a total design capacity of $60 \mathrm{~kW}$ of heat removal [5]. In its present form, the cooling uses a conventional compression $\rightarrow$ condensation $\rightarrow$ detent cycle but the oil-free compressor plant is currently being replaced with a passively pumped thermosiphon [6]. The thermosiphon system uses the $92 \mathrm{~m}$ height difference between the surface and the ATLAS underground experimental caverns, in combination with the thermodynamic cycle, to drive the coolant circulation. In this design, the necessary liquid delivery pressure will be provided by the hydrostatic column of $92 \mathrm{~m}$ of $\mathrm{C}_{3} \mathrm{~F}_{8}$ liquid, while the $\mathrm{C}_{3} \mathrm{~F}_{8}$ vapor will climb to the surface condenser, the lowest pressure element in the system. The headspace above the coolant in the liquid reserve vessel is also at sub-atmospheric pressure and will need to be monitored for the ingress of air. A passive ultrasonic leak detection system is ideal in this application to respect the purity of the gas circulating in the system.

Figure 1. The speed of sound in a gas mixture can be very sensitive to the ratio of the two components, depending on the difference in their molecular masses.

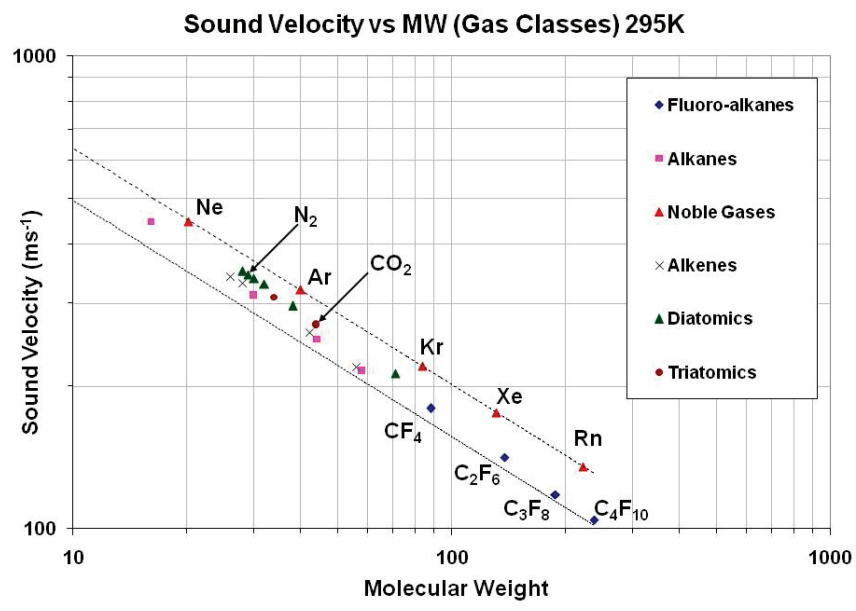

The aforementioned silicon detector systems had been suspected of leaking small amounts of coolant into each of their segregated, nitrogen-flushed environments from the time of their installation in 2006-2007. The possible leaks are of concern because the high ionizing radiation fluence of the ATLAS inner detector environment could dissociate $\mathrm{C}_{3} \mathrm{~F}_{8}$ coolant molecules, 
possibly leading to the creation of hydrofluoric acid (HF) if it should happen that a detector gas volume were to be accidentally exposed to humidity during detector operation. This could lead to corrosion damage to the (millions of) exposed Al wire bonds (wire bonds are very thin, typ. 1 mil, for short connections between circuit components, most usually between an integrated circuit and an interconnect, such as a printed circuit board or standardized package.) used in the detectors, rendering the associated detector modules with limited or no functionality, creating (a) blind spot(s) in the Inner Detector of the ATLAS experiment. Because the detector volumes of the Pixel and SCT sub-detectors are separately flushed with $\mathrm{N}_{2}$ to prevent condensation, the possibility exists to extract and analyze each volume exhaust for coolant leaks.

In this paper we describe how the application of existing detector technology for ultrasonic fractional binary gas analysis in new hardware and control systems can provide sensitive, in situ, passive leak detection. We also describe design options that give the ultrasonic sensing system additional functionality.

\section{Theory, Design and Implementation of Ultrasonic Leak Detection in Binary Gas Systems}

The principle of operation and implementation of the ultrasonic leak detection system is described in the following three subsections.

\subsection{Principle of Operation}

The principle for leak detection using ultrasonic acoustic waves is based on the general equation for sound velocity in a gas:

$$
c=\sqrt{\frac{\gamma R T}{M}}
$$

where $\gamma$ is the adiabatic index of the gas, $R$ is the molar gas constant $\left(8.3145 \mathrm{~J} \cdot \mathrm{mol}^{-1} \cdot \mathrm{K}^{-1}\right), T$ is the absolute temperature in degrees Kelvin and $M$ is the molar mass in $\mathrm{kg} \cdot \mathrm{mol}^{-1}$. The value of $\gamma$ is given by the ratio of the molar specific heat at constant pressure $(C p)$ to that at constant volume $(\mathrm{Cv})$ :

$$
\gamma=\frac{C p}{C v}
$$

When dealing with a gas mixture, one calculates the sum of the specific heat components in proportion to the molar fraction, $w_{\mathrm{i}}$, of each:

$$
\gamma_{m}=\frac{C_{p m}}{C_{v m}}=\frac{\sum_{i} w_{i} C p_{i}}{\sum_{i} w_{i} C v_{i}}
$$

By definition, the molar mass of the mix is given by:

$$
M=\sum_{i} w_{i} M_{i}
$$

So Equation (1) becomes: 


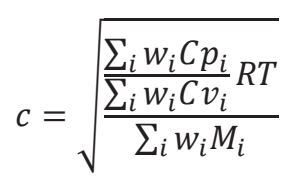

For each mixture component, the molar specific heat at constant pressure and that at constant volume are related by:

$$
C p_{i}-C v_{i}=R
$$

The precision of mixture determination, $\partial(\mathrm{mix})$, at any concentration of the two components is given by:

$$
\partial(\operatorname{mix})=\frac{\partial c}{m}
$$

where $m$ is the local slope of the sound velocity-concentration curve and $\partial c$ is the uncertainty in the sound velocity measurement.

In a system composed of $\mathrm{N}_{2}$ and $\mathrm{C}_{3} \mathrm{~F}_{8}$, for example, the mixture ratio can be expressed as $w_{N 2}=\left(1-w_{C 3 F 8}\right)$, reducing Equation (5) to a function of $w_{C 3 F 8}, C p_{C 3 F 8}$ and $C_{p_{N 2}}$. The two molar specific heats can be computed using NIST REFPROP [7] or with PC-SAFT [8] over a range of temperature and pressure exceeding the conditions in the analyzer tube(s) and then stored in a database (look-up table). Therefore, we only need measure the speed of sound in the mixture along with the temperature profile along the path of the gas (for improved accuracy) and the gas pressure, in order to calculate $w_{C 3 F 8} / w_{N_{2}}$.

\subsection{Implementation}

In our system designs, ultrasonic bursts are propagated in a sealed tube designed to provide a smooth flowing gas region between two transducers (Figure 2). The transducers were designed by Polaroid $^{\circledR}$ and are now sold by Senscomp (Figure 3) as series 600 [9]. The transducer is comprised of a thin, Au plated Mylar ${ }^{\circledR}$ foil stretched over a spirally grooved conductive disk. The foil is held at ground potential and the disk is biased between $100 \mathrm{Vdc}$ and $360 \mathrm{Vdc}$. A $50 \mathrm{kHz}$ pulse train modulates the transducer bias voltage, exciting the diaphragm to transmit an acoustic wave in response to the fluctuating electric field. As a sensor, the transducer collects an alternating charge across the foil-disk capacitor that is proportional to the power of the signal impinging on the foil diaphragm. A $50 \mathrm{Vdc}$ bias is adequate in receive mode to maximize the signal to noise ratio of the transducer in the gases we have studied. An appealing feature of this sensor is that its construction maintains pressure equilibrium across the diaphragm, making the pressure in the gas system irrelevant to transducer operation. Its suitability for use with corrosive gases has not been examined but these sensors have displayed long lifetimes in the applications reported here (none are known to have failed).

Most of the electronics needed to drive the transducers, create gated pulses and measure the time of flight of pulses between the transmit and receive transducers can be implemented in a micro-controller. The Microchip ${ }^{\circledR}$ dsPIC 16 bit microcontroller has been chosen for the present ATLAS design. It provides a $40 \mathrm{MHz}$ timer for measurement of the sound transit time through the 
gas or gas mixture that is started when the first ultrasonic pulse is sent. The microcontroller also controls the gain of the analog amplifier and generates an analog comparison voltage for the discriminator, whose output signal triggers the $40 \mathrm{MHz}$ timer to halt. The discriminator follows the analog amplifier to boost the received transducer signal and shape it. It is also possible to design the system to take alternating measurements in opposite directions, relative to the gas stream, so that the analog amplifier, cabling and discriminator delays are eliminated from the time of flight measurements. This technique also allows passive gas velocity measurement in the same instrument that is used for leak detection [10].

Figure 2. An illustration of the ultrasonic signal transit time measurement and the electronic readout block diagram is shown for a design that allows the transducer roles to be swapped for more precise calibration and for simultaneous gas velocity measurement.

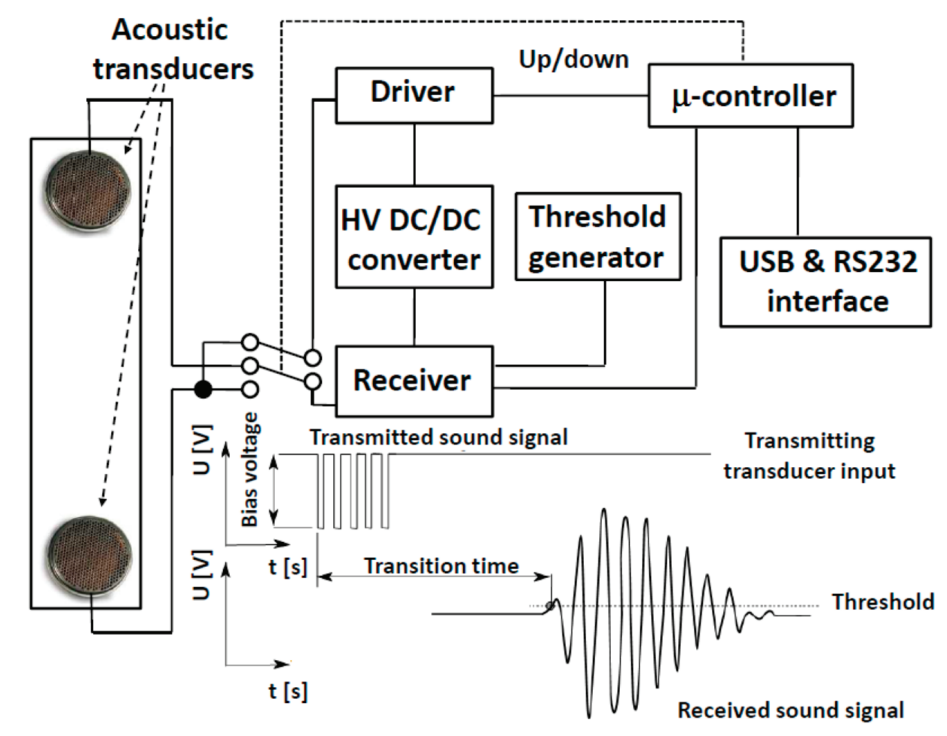

Figure 3. Senscomp model 600 capacitive ultrasonic transducer originally designed by Polaroid ${ }^{\circledR}$ for autofocus cameras.

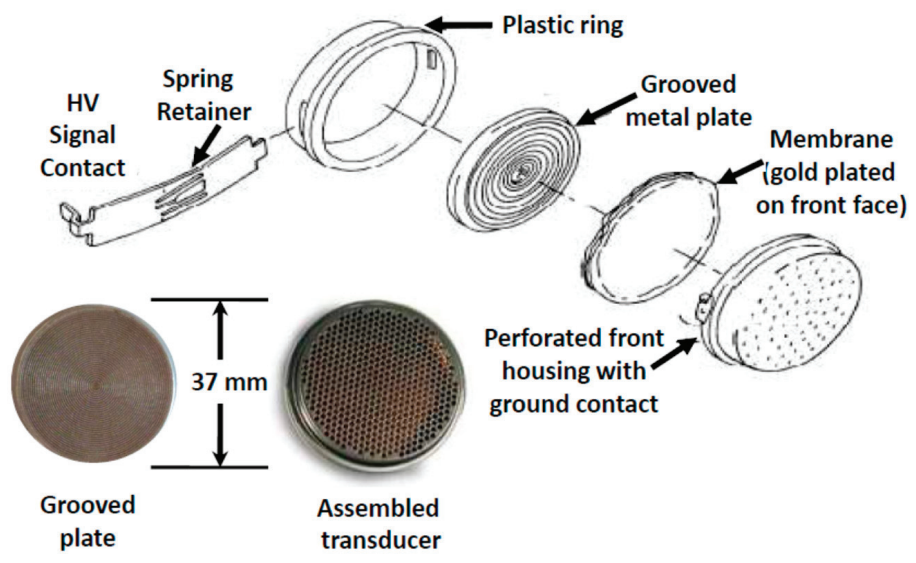


It is possible to use the same microcontroller to store the sound velocity-composition lookup tables and locally calculate the mixture ratio. However, ATLAS is structured around a control and monitoring system that makes it more practical to perform lookup and other functions in a supervisory computer that can serve several analyzer tubes. Communications protocols are determined by the specific application. In ATLAS, we will convert to MODBUS over TCP/IP but have also used and will continue to use RS-232C and USB for local digital communication and 4-20 mA current loops for hard-wired control functions.

The gas mixture and flow rate are continuously calculated using Supervisory Control and Data Acquisition (SCADA) software implemented in Siemens WinCC ${ }^{\circledR}$ (formerly known as PVSS II [11]), running on the supervisory computer. Sound velocity-concentration lookup tables are used to accelerate execution speed and are stored on the same computer. These tables may be created from prior measurements in calibration mixtures, theoretical thermodynamic calculations, published tables or a combination of these sources.

After completion of the full ultrasonic analysis network in 2014, a central computer will be used to supervise the four leak check systems described in this paper, as well as another ultrasound system for passively measuring the gas flow velocity in the thermosiphon vapor returning to the surface condenser, which operates at sub-atmospheric pressure [12].

The acoustic wave has a very low angle of dispersion at $50 \mathrm{kHz}$ because it is driven from a diaphragm that is much larger (typically by a factor of 10) than the acoustic wavelength of the probe signal. The important features of a tube for ultrasonic gas analysis include mounts for the transducers that keep the transducer diaphragms aligned parallel to each other in the gas stream. Misalignment can lead to increased errors due to reflections from the tube walls and/or attenuation of the signal. Since the wavelength of the ultrasonic signals is very short, the choice of length for the measurement tube is driven by other considerations, including mounting space and the precision of the timer(s) used to measure the time of flight of the probe signal. Provision should also be made to seal the transducer wiring ports, including the wiring for any temperature and pressure sensors that might be included inside the tube for improved accuracy. Care should be taken to avoid creating acoustic resonators or vortices in the analyzer tube design that could interfere with the measurement. The tube should be installed in a system line that is free of liquid, aerosols, risk of condensation and other contaminants.

A tube design allowing simultaneous binary gas analysis and velocity measurement (flowmetry), has been modeled using computational fluid dynamics [13]. One such tube design for axial flowmetry is shown in Figure 4. Note the cones that reduce chaotic fluid flow and vortices as gas flows around the transducers. Although stainless steel is chosen for the analyzer tube construction in our applications, other materials may be more suitable in others. The design should anticipate pipe stems, valves and manifolds to ease calibration, servicing and replacement of the sensing tube and/or elements within it.

As mentioned in the introduction, it is possible to monitor the $\mathrm{N}_{2}$ environmental volumes of the ATLAS inner detector for $\mathrm{C}_{3} \mathrm{~F}_{8}$ leaks by remotely sampling their atmospheres. Dry $\mathrm{N}_{2}$ is constantly flowing to purge the detector volumes of any humidity, and gas can be aspirated from different locations through seven $8 \mathrm{~mm}$ diameter exhaust tubes. These tubes traverse a path of $150 \mathrm{~m}$ length 
from the ATLAS Inner Detector to three ultrasonic analyzers installed in an underground service cavern, a safe distance from the high radiation environment of the experimental cavern. One of these tubes is dedicated to sampling the environmental volume of the ATLAS Pixel Detector and two others sample the ATLAS SCT Detector. Another tube is routed to a soon-to-be-installed new silicon pixel detector, known as the "Insertable B Layer" (IBL), equipped with its own separately purged $\mathrm{N}_{2}$ environmental volume. Since the IBL will be evaporatively cooled using a new $\mathrm{CO}_{2}$ based system, we are extending the analysis capability of the ultrasonic instrument to include $\mathrm{CO}_{2}$ leaks into $\mathrm{N}_{2}$ [14].

Figure 4. The schematics show the implementation of a stainless steel analyzer tube, with the transducer highlighted in gold in bottom detail. The central tube inner diameter is pinched to be the same as the ultrasonic transducers. This version allows volume flow measurement to $\sim 230 \mathrm{~L} \cdot \mathrm{min}^{-1}$, in addition to the measuring the gas mixture ratio.

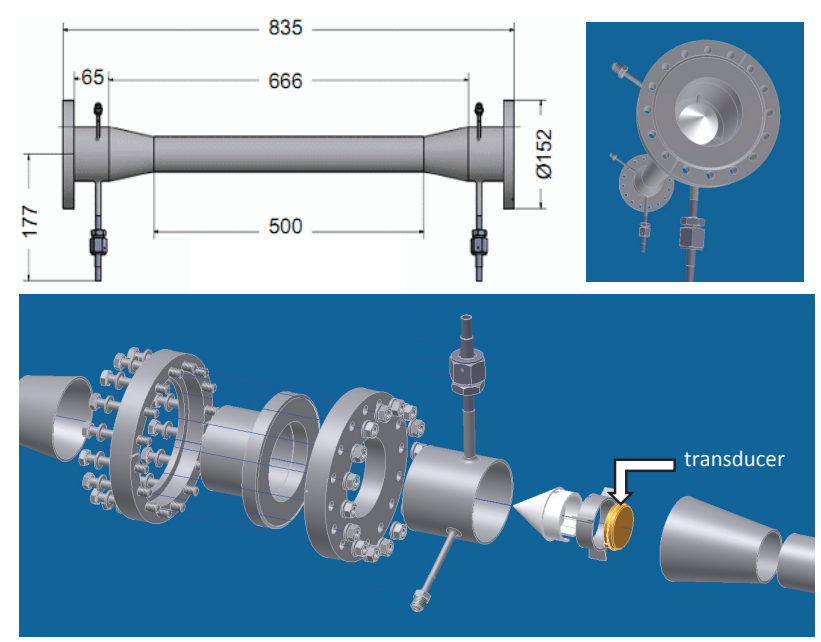


Figure 5. Schematic of the ATLAS inner detector leak detection system. Two ultrasonic analyzer tubes target detection of $\mathrm{C}_{3} \mathrm{~F}_{8}$ leaks into the $\mathrm{N}_{2}$ environments surrounding the Pixel and SCT detectors. A third tube targets $\mathrm{CO}_{2}$ leaks into the IBL gas volume. A portable gas chromatograph (GC) can be connected as a calibration check or if more than one contaminating gas is suspected [15].

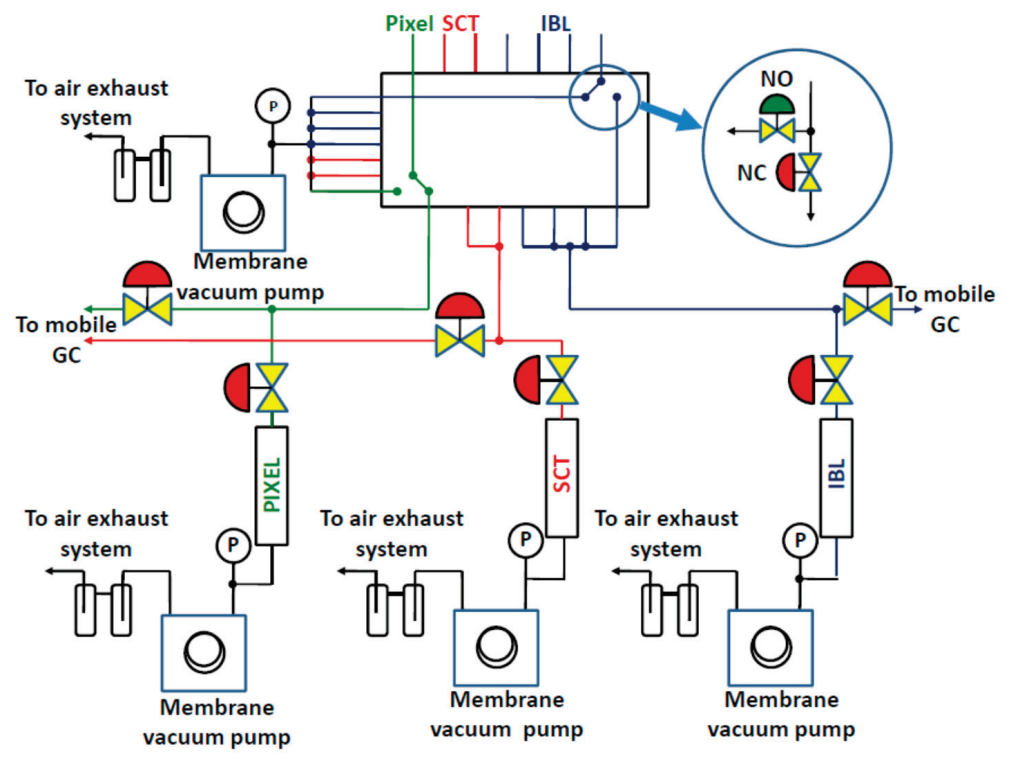

Figure 5 illustrates the gas sampling system in which gas is pumped through three ultrasonic gas analysis tubes using low volume flow $\left(\sim 100 \mathrm{~cm}^{3} \cdot \mathrm{min}^{-1}\right)$ diaphragm pumps. A pneumatic valve selection panel located in the service cavern allows the operator to remotely choose (or program) which detector volumes to analyze.

In the thermosiphon condenser application, a vertical tube system is required that can be isolated from the condenser for maintenance and calibration. Fortunately this is simplified by being a part of the head-space purging system of the coolant reserve tank, which can be isolated from the rest of the tank (Figure 6). The condenser is located outdoors, far from rack space for computers and other equipment, so for this system, the electronics have been implemented within a DIN rail compliant weatherproof equipment box. Communication will be by MODBUS over TCP/IP to the supervisory computer, which is the same one used for leak detection, located in an equipment rack with the three analyzer tubes mentioned previously. 
Figure 6. The schematic and logic flow of the leak detection system of the condenser headspace of the ATLAS inner detector thermosiphon cooling system is shown on the left. A valve system is included which allows for filling the analyzer tube with a calibration gas and purging the tube with a vacuum pump. The completed analyzer and valve tree is shown on the right, prior to installation.
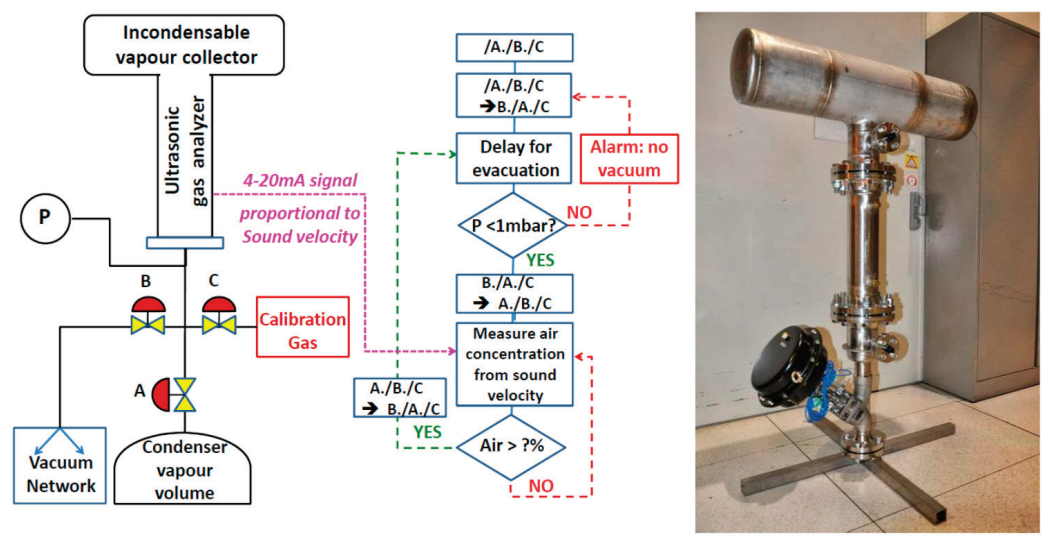

\subsection{Calibration}

Precision measurement of the mixture is achieved by accurately determining the distance between the transducers, i.e., calibrating the analyzer tube length. The sound transit time can be calibrated by filling the analyzer tube with a pure gas. The best candidate gases are those with well-known sound velocity dependence on temperature and pressure, including xenon, whose sound velocity $\left(175.5 \mathrm{~ms}^{-1}\right.$ at $\left.20^{\circ} \mathrm{C}\right)$ is the closest to that of the fluorocarbon gases analyzed in ATLAS and for which the thermo-physical behavior is that of an ideal gas. Sufficient precision is often possible using nitrogen or argon, which are considerably cheaper and more widely available. The average uncertainty in transducer inter-distance measured in this way is $\pm 0.1 \mathrm{~mm}$. Of obvious importance to calibration is control of the temperature and pressure or at least, their accurate measurement (Equations (1)-(6)). For even tighter calibration, the design can include a thermal jacket around the analyzer tube. In the instruments we have deployed, the combined effects of uncertainties in the transducer spacing and the measured temperature $\left(< \pm 0.2{ }^{\circ} \mathrm{C}\right)$ and pressure $(< \pm 4$ mbar) in the tube result in an uncertainty in sound velocity measurement of $< \pm 0.05 \mathrm{~ms}^{-1}$. In combination with the stored sound velocity-concentration look up tables, this results in an uncertainty of the gas mixture determination, calculated with Equation (7), depending on the molecular weight difference of the two components, as discussed in the following sections.

\section{Results and Discussion}

\subsection{Examples of Velocity-Concentration Look-UP Tables}

Figures 7 and 8 illustrate look up tables applicable to leak detection of $\mathrm{C}_{3} \mathrm{~F}_{8}$ into $\mathrm{N}_{2}$. Figure 7 compares measured sound velocities in molar concentrations of up to $10 \% \mathrm{C}_{3} \mathrm{~F}_{8}$ in $\mathrm{N}_{2}$ with direct 
sound velocity predictions made using the PC-SAFT equations of state [8]. The data of Figure 7 are abstracted from measurements and predictions made over the full concentration range from pure $\mathrm{C}_{3} \mathrm{~F}_{8}$ to pure $\mathrm{N}_{2}$. The PC-SAFT approach was used, as NIST-REFPROP is not presently configured for direct sound velocity calculations for mixtures of saturated fluorocarbons with $\mathrm{N}_{2}$. In an alternative approach illustrated in Figure 8, sound velocity may be determined using the formalism of Equations (1)-(6) using $\mathrm{C}_{\mathrm{p}}$ and $\mathrm{C}_{\mathrm{v}}$ data for the two component gases separately calculated using NIST-REFPROP over a range of temperature and pressure encompassing the process gas conditions.

Figure 7. Comparison of sound velocity measurements and PC-SAFT predictions in $\mathrm{C}_{3} \mathrm{~F}_{8} / \mathrm{N}_{2}$ mixtures at 1 bar abs and $25{ }^{\circ} \mathrm{C}$ up to $10 \%$ molar concentration.

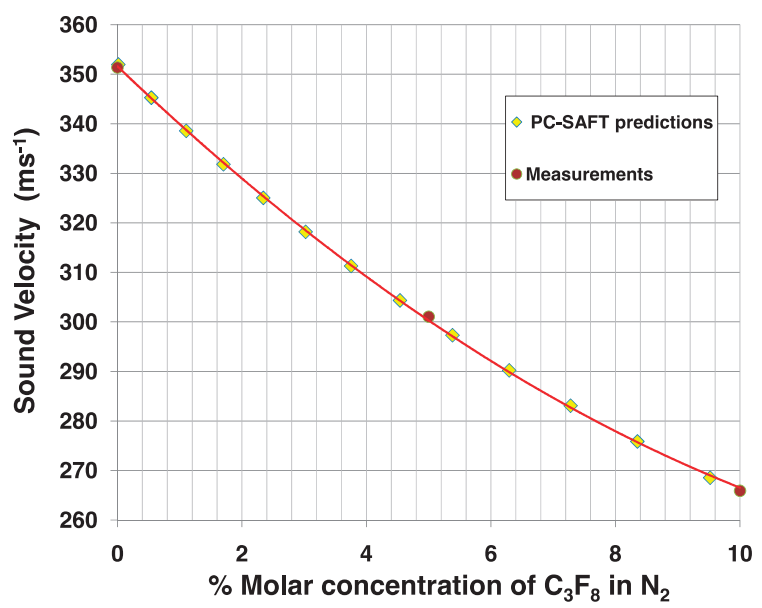

The large difference in molecular weight between $\mathrm{C}_{3} \mathrm{~F}_{8}$ and $\mathrm{N}_{2}$ (respectively 188 and 28 units) affords very high sensitivity to variations in the $\mathrm{C}_{3} \mathrm{~F}_{8}$ leak concentration. For example in the ( $0 \%$ $1 \%$ ) molar concentration (MC) range of most interest in leak detection of a heavy vapor into a light carrier, the slope of the sound velocity-concentration curve is $-12.2 \mathrm{~ms}^{-1} .(\% \mathrm{MC})^{-1}$. This slope, taken in combination with the sound velocity measurement error of $\pm 0.05 \mathrm{~ms}^{-1}$ using Equation (7), results in a mixture resolution of $\pm 4 \times 10^{-5}$. 
Figure 8. Sound velocity calculated according to the formalism of Equations (1)-(6) for $\mathrm{C}_{3} \mathrm{~F}_{8} / \mathrm{N}_{2}$ molar concentrations in the range $\left(0 \%-1 \% \mathrm{C}_{3} \mathrm{~F}_{8}\right)$ at 1 barabs and several temperatures.

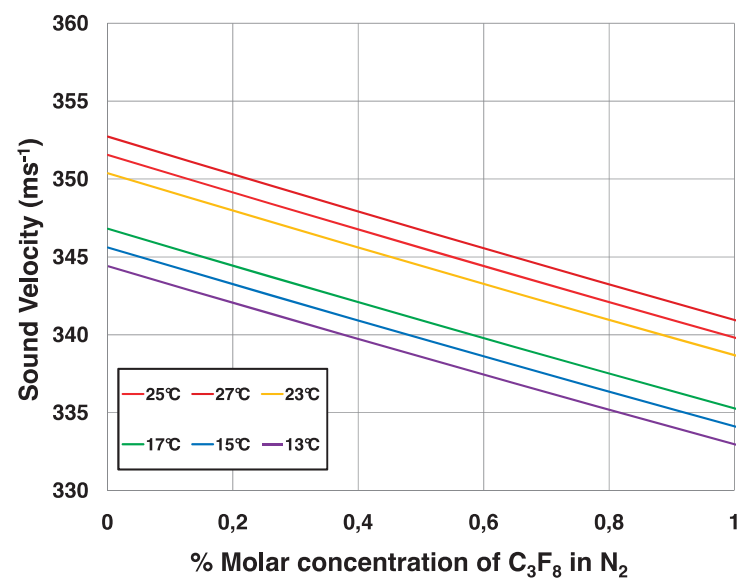

Figure 9 illustrates the variation of sound velocity with the concentration of $\mathrm{CO}_{2}$ leaking into a $\mathrm{N}_{2}$ atmosphere, as in the case of the environmental volume of the new ATLAS IBL. Here the smaller difference in molecular weight between $\mathrm{CO}_{2}$ and $\mathrm{N}_{2}$ (respectively 44 and 28 units) results in a shallower slope to the sound velocity-concentration curve. For example in the $(0 \%-0.1 \%)$ molar range of most interest in leak detection the slope of the sound velocity-concentration curve is $-1.12 \mathrm{~ms}^{-1}(\% \mathrm{MC})^{-1}$. This slope, taken in combination with the sound velocity measurement error of $\pm 0.05 \mathrm{~m} / \mathrm{s}$ using Equation (7), results in a mixture resolution of $\pm 4.4 \times 10^{-4}$.

Figure 9. Sound velocity calculated according to the formalism of Equations (1)-(6) for $\mathrm{CO}_{2} / \mathrm{N}_{2}$ mixtures in the molar concentration range $\left(0 \%-0.1 \% \mathrm{CO}_{2}\right)$ at 1 barabs and a tube operating temperature of $21.8^{\circ} \mathrm{C}$.

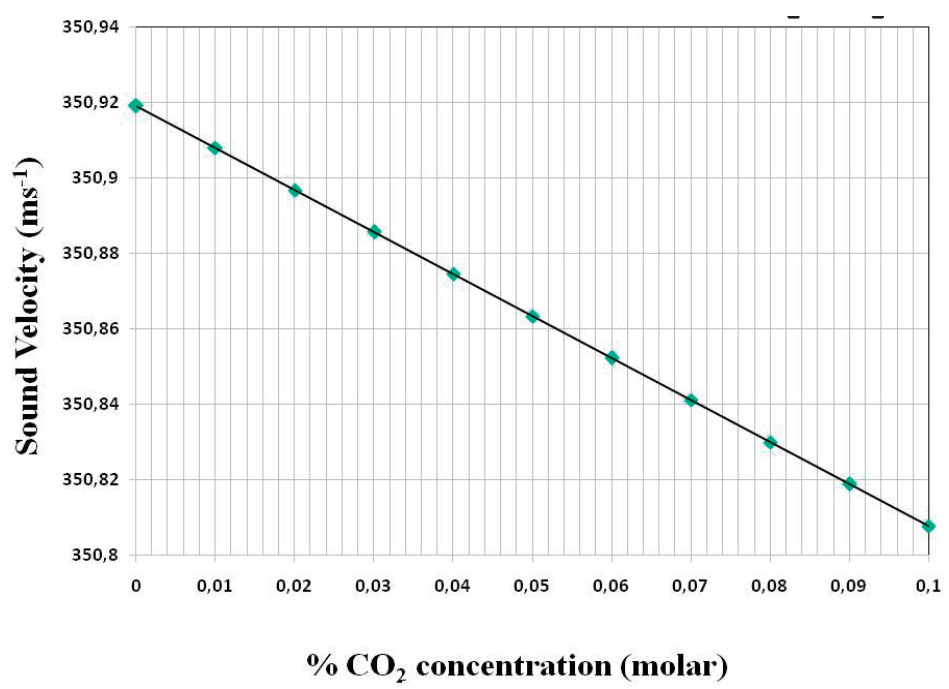


Figure 10 illustrates the variation of sound velocity with the concentration of air leaking into the $\mathrm{C}_{3} \mathrm{~F}_{8}$ atmosphere in the headspace of the thermosiphon condenser. Although the difference in molecular weight between $\mathrm{C}_{3} \mathrm{~F}_{8}$ and air (respectively 188 and 29 units) is still very large, this instrument operates at the opposite end of the spectrum to the example shown in Figures 7 and 8.

Figure 10. The sound velocity is calculated according to the formalism of Equations (1)-(6) for $\mathrm{C}_{3} \mathrm{~F}_{8}$ /air mixtures in the molar concentration range ( $0 \%-30 \%$ air) at $1 \mathrm{bar}_{\mathrm{abs}}$ at three tube operating temperatures [16].

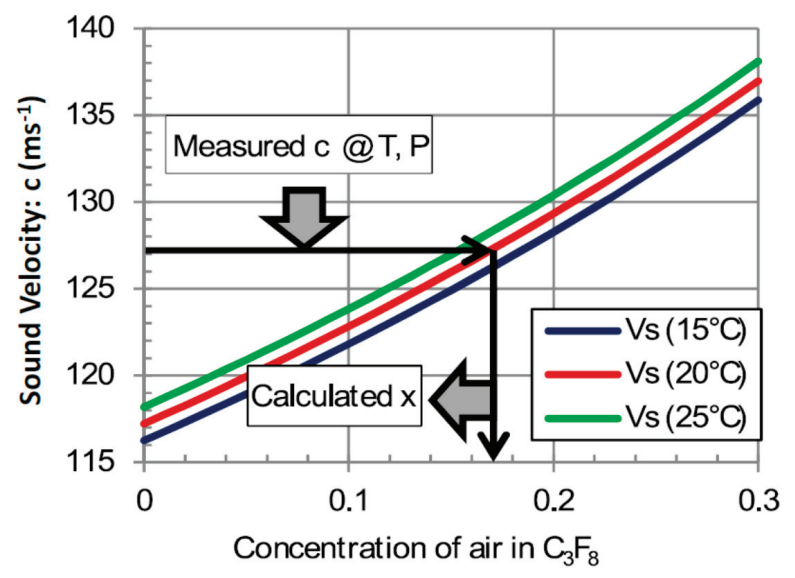

In this case, a light contaminant leaks into a heavy carrier. The slope for the corresponding end of the velocity-concentration curve is shallower, e.g., in the $(0 \%-10 \%)$ molar range of most interest in this application the slope of the sound velocity-concentration curve is $0.53 \mathrm{~ms}^{-1}(\% \mathrm{MC})^{-1}$. This slope, taken in combination with the sound velocity measurement error of $\pm 0.05 \mathrm{~ms}^{-1}$ and using Equation (7), results in a mixture resolution of $\pm 9.4 \times 10^{-4}$.

\subsection{Results from Operation of the Devices}

The $\mathrm{N}_{2}$ atmosphere surrounding the ATLAS pixel detector was continuously monitored from March 2011 until the programmed 18-month shutdown of the CERN LHC in February 2013. Gas was aspirated from the pixel detector volume at around $100 \mathrm{~mL} / \mathrm{min}$ with the analyzer tube operating at a pressure of $(985 \pm 2)$ mbarabs and a typical operating temperature of $15.2{ }^{\circ} \mathrm{C}$. The $\mathrm{C}_{3} \mathrm{~F}_{8}$ concentration as a function of time is shown in Figure 11. The electronics had several downtimes of a few weeks, which caused the discontinuity in the distribution. The short periods of drops to zero $\mathrm{C}_{3} \mathrm{~F}_{8}$ concentration correspond to periodic baseline checks with pure $\mathrm{N}_{2}$. Longer period fluctuations are due to variations in the nitrogen purge rate through the detector environmental volume. The peak in apparent $\mathrm{C}_{3} \mathrm{~F}_{8}$ concentration seen in November 2012 was due to the simultaneous aspiration of air into the tube through an improperly tightened gas fitting, the apparent $\mathrm{C}_{3} \mathrm{~F}_{8}$ concentration being based on the increased sound transit time and correspondingly reduced sound velocity in the tube. A reduction in sound velocity of $0.86 \mathrm{~ms}^{-1}$ from that of pure nitrogen is typically observed when the full Pixel Detector cooling system of 88 independent circuits is fully 
operating. From the $\sim 12.27 \mathrm{~ms}^{-1}$. $(\% \mathrm{MC})^{-1}$ average gradient of the sound velocity-concentration curve for $\mathrm{C}_{3} \mathrm{~F}_{8}$ concentrations in the range $0 \%-0.5 \%$ (Figures 7 and 8 ) this sound velocity difference indicates, via Equation (7), a $\mathrm{C}_{3} \mathrm{~F}_{8}$ ingress of $0.07 \%$ (Figure 11).

Both the pressure and the temperature of the gas in the tube are continually measured electronically, and a full interpolation is made between points of the created (c; P; T; $\mathrm{C}_{3} \mathrm{~F}_{8}$ concentration) database.

Gas was aspirated from two different zones of the SCT detector $\mathrm{N}_{2}$ envelope into an analysis tube that was operated for three weeks in February 2013 with the latest version of the electronics, until the LHC shutdown. Figure 12 illustrates the concentrations of $\mathrm{C}_{3} \mathrm{~F}_{8}$ during that period. Through the use of the automated sampling system of Figure 5, gas was alternately sampled for a period of one hour from each zone. The variance between the two zones (upstream and downstream into the SCT environmental volume with respect to the direction of dry nitrogen gas injection) was seen to diminish after 15 February when the purge rate was increased.

ATLAS commenced a long (2013-2014) maintenance and upgrade shutdown, ceasing operations in mid-February 2013. On the 20th of that month the cooling circuits of the ATLAS silicon detectors were progressively turned off, starting with those of the SCT and followed by those of the Pixel detectors. The cooling shutdown and elimination of $\mathrm{C}_{3} \mathrm{~F}_{8}$ from the cooling circuits was complete by 00:00 on February 21. Before the start of this process, an № baseline was taken for the instrument analyzing the pixel detector environmental gas.

Figure 11. This plot of the long duration ( $>18$ month) $\log$ of $\mathrm{C}_{3} \mathrm{~F}_{8}$ leak contamination in the $\mathrm{N}_{2}$ environmental gas surrounding the ATLAS Pixel Detector confirms suspected leaks and establishes the leak rate into the detector volume [12].

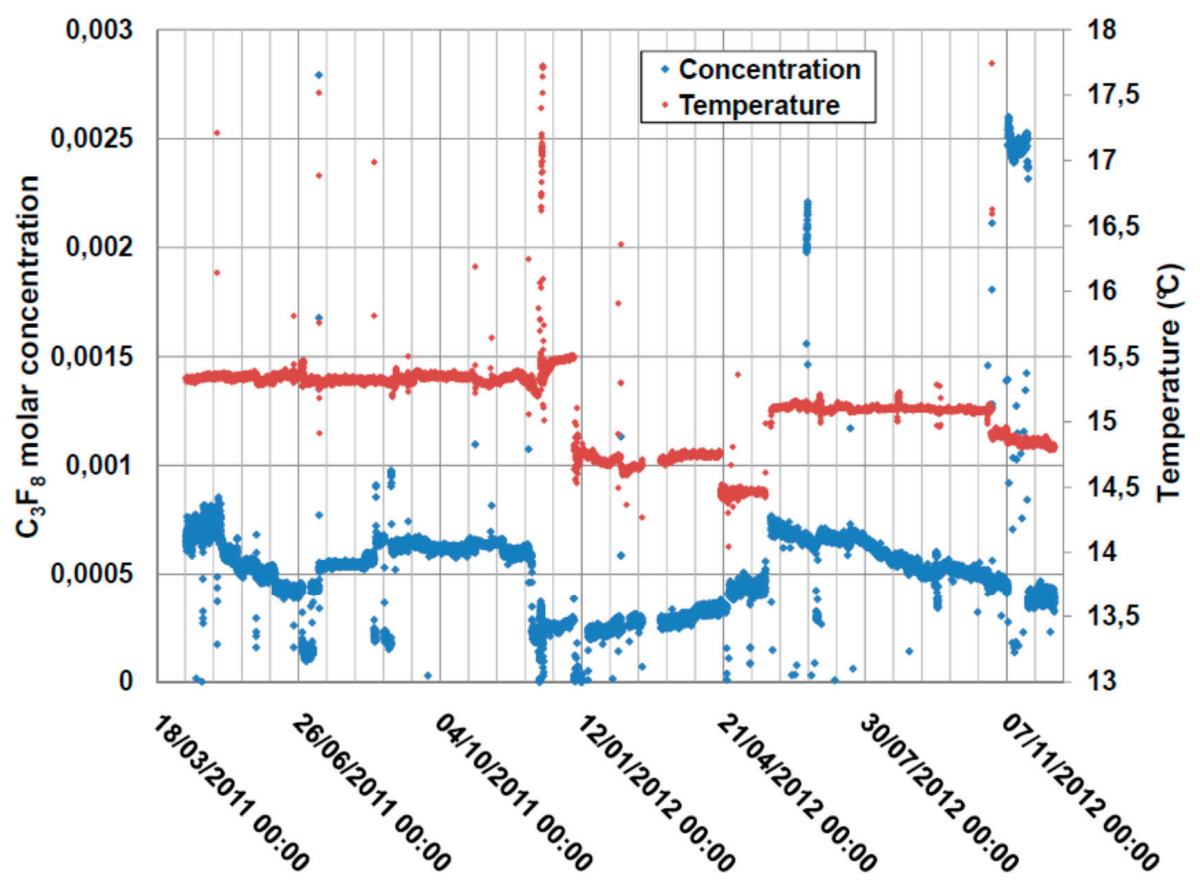


Figure 12. The variance in concentration of $\mathrm{C}_{3} \mathrm{~F}_{8}$ in the SCT gas enclosure as a function of time (between 1st and 21st February) is shown for the two zones analyzed, designated SCTA and SCTC [12].

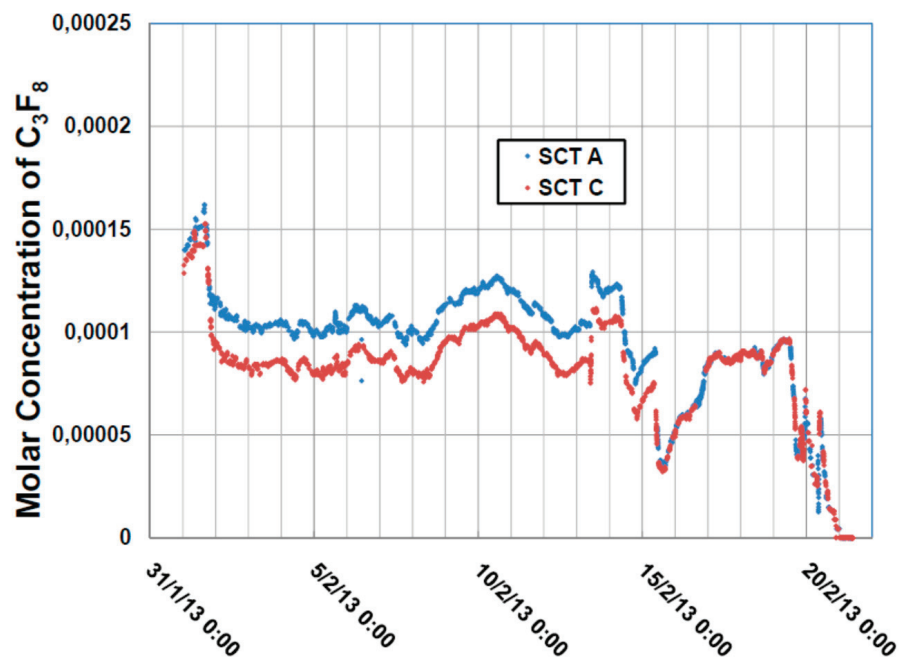

Figure 13. The variance in concentration of $\mathrm{C}_{3} \mathrm{~F}_{8}$ is shown for the Pixel (right) and SCT (left) gas enclosures during the shutdown of the cooling systems on 20th February 2013, the latter of which was regularly alternated between the SCTA and SCTC zones during the shutdown [12].

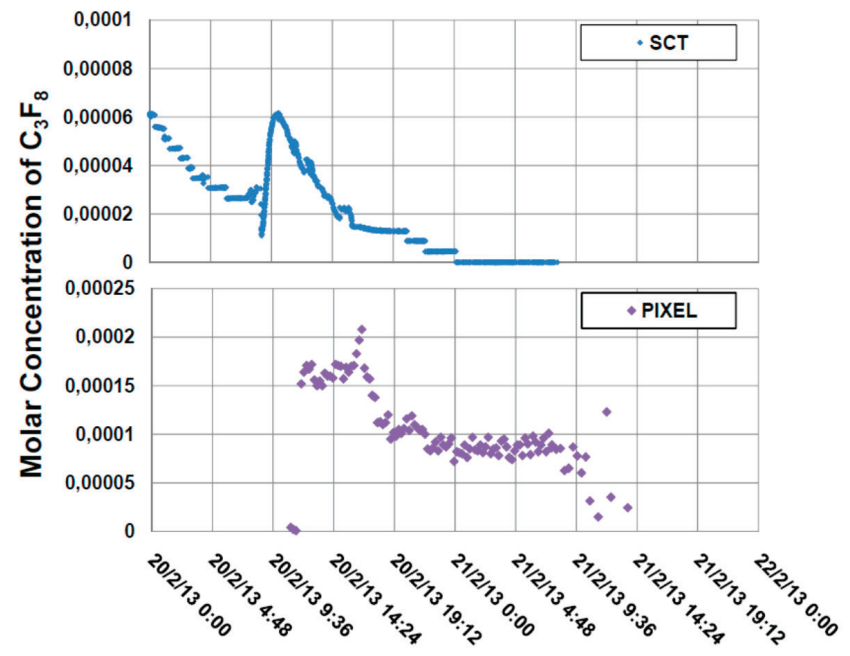

The residual $\mathrm{C}_{3} \mathrm{~F}_{8}$ contamination in both zones of the SCT $\mathrm{N}_{2}$ envelope was seen to drop to zero within around $15 \mathrm{~h}$ of the commencement of the SCT cooling shutdown. The Pixel Detector cooling shutdown protocol was longer due to known leaks in some of the cooling circuits. Residual $\mathrm{C}_{3} \mathrm{~F}_{8}$ thus continued to be aspirated from the pixel detector nitrogen envelope over a longer period. 
The largest drops in the $\mathrm{C}_{3} \mathrm{~F}_{8}$ concentration were seen following the shutdown of the 3rd pixel barrel layer, which began at 17:00 on 20 February 2013 (Figure 13).

\section{Conclusions and Outlook}

We have described an ultrasonic instrument for continuous real-time binary gas composition measurement, through the combination of sound velocity, temperature and pressure measurements. The instrument is particularly well suited to measure leaks of a high molecular weight gas into a light carrier. A sensitivity of $<5 \times 10^{-5}$ has been demonstrated to leaks of octoflouropropane $\left(\mathrm{C}_{3} \mathrm{~F}_{8}\right)$ coolant into nitrogen during a long duration (18 month) continuous study. Sensitivity studies suggest that mixture resolutions of $<9.5 \times 10^{-4}$ and $<4.5 \times 10^{-4}$ respectively will be possible in $\mathrm{C}_{3} \mathrm{~F}$ / air and $\mathrm{CO}_{2} / \mathrm{N}_{2}$ mixtures of interest to the ATLAS inner detector cooling project. Although this development was motivated by the requirements of an evaporative cooling system, the instrument is applicable to the detection of leaks of many other refrigerants, and to processes requiring continuous knowledge of binary gas composition.

The Pixel and SCT volumes continue to be monitored for leaks as part of a large system that monitors the health of the ATLAS detectors. The accuracy of passive, ultrasonic leak detection has already proven its usefulness in understanding the coolant leaks in the ATLAS silicon detectors.

Operation of the condenser headspace monitoring system we have described will begin during commissioning of the new thermosiphon cooling system for the ATLAS silicon detectors during 2014. Installation has begun for the plumbing necessary to instrument the new IBL Detector volume for monitoring. Since $\mathrm{CO}_{2}$ is known to be highly absorbent of ultrasonic and even high audio frequencies, a new R\&D program has begun to explore the severity of attenuation at high $\mathrm{CO}_{2}$ concentrations. Preliminary studies suggest that $50 \mathrm{kHz}$ signals can be detected in our present instrument after passage through $50 \mathrm{~cm}$ of gas at molar $\mathrm{CO}_{2}$ concentrations up to $15 \%$. We therefore also plan to investigate the use of audio frequency transducers in a new system being built at the University of Oklahoma. The results obtained will allow optimization of the tube geometry and choice of transducers in the ATLAS IBL application.

Additional uses for speed of sound measurements in parallel with the work described here include gas flowmetry and analysis of $\mathrm{C}_{2} \mathrm{~F}_{6} / \mathrm{C}_{3} \mathrm{~F}_{8}$ coolant blends for optimized thermodynamics [2].

The technology described in this work could also find application in other sectors where passive, long term, in situ leak detection (or fractional gas mixture analysis) is required. These include Metal Organic Chemical Vapor Deposition (MOCVD) semiconductor manufacturing, anesthesiology and hydrocarbon fuel combustion management.

\section{Acknowledgments}

The authors wish to thank CERN, ATLAS [17] and their home institutes for support of this project. University of Indiana participation was supported through U.S. Department of Energy contract DE-AC02 98CH 10886. University of Oklahoma participation was supported through DOE contract DEFG02-04ER41305. Alexander Bitadze acknowledges support for his Ph.D. research from the ATLAS inner detector group and the particle physics group of the University of Glasgow. 
Richard Bates. acknowledges support from the U.K. Science and Technology Facilities Council. Martin Doubek, Michal Vitek and Vaclav Vacek acknowledge individual support and support for this project through the following grants in the Czech Republic: MSM grants No. 6840770035 \& LA08015 and SGS/FIS grant No. 10-802460 of the Czech Technical University, Prague.

\section{Conflicts of Interest}

The authors declare no conflict of interest.

\section{References}

1. Hallewell, G.; Crawford, G.; McShurley, D.; Oxoby, G.; Reif, R. A Sonar-based instrument for the ratiometric determination of binary gas mixtures. Nucl. Instr. Meth. A 1988, 264, 219.

2. Bates, R.; Battistin, M.; Berry, S.; Berthoud, J.; Bitadze, A.; Bonneau, P.; Botelho-Direito, J.; Bousson, N.; Boyd, G.; Bozza, G.; et al. A combined ultrasonic flow meter and binary vapour mixture analyzer for the ATLAS silicon tracker. JINST 2013, doi:10.1088/17480221/8/02/P02006.

3. The ATLAS Collaboration. The Pixel Detector Technical Design Report; CERN-LHCC-9813; LHCC, CERN, TDR: Geneva, Switzerland, 1998.

4. The ATLAS Collaboration. Inner Detector Technical Design Report; CERN-LHCC-97016\&017; LHCC, CERN, TDR: Geneva, Switzerland, 1997.

5. Attree, D.; Anderson, B.; Anderssen, E.; Akhnazaro, V.; Apsimon, R.J.; Barclay, P.; Batchelor, L.E.; Bates, R.L.; Battistin, M.; Bendotti, J.; et al. The evaporative cooling system for the atlas inner detector. JINST 2008, doi:10.1088/1748-0221/3/07/P07003.

6. Botehlo-Direito, J.; Perez Rodriguez, E.; Egorov, K.; Zwalinski, L.; Bitadze, A. Technical_Note_60kW_Thermosiphon; CERN EDMS: Geneva, Switherland, 2010; Available online: https://edms.cern.ch/document/1083852/1 (accessed on 19 June 2014).

7. Lemmon, E.; Huber, M.; McLinden, M. REFPROP Standard Reference Database 23, version 9.0; standard; National Institute of Standards and Technology: Gaithersburg and Boulder, MD, USA, 2010.

8. Gross, J.; Sadowski, G. Perturbed-Chaim SAFT: An equation of state based on a perturbation theory for chain molecules. Ind. Eng. Chem. Res. 2001, 40, 1244-1260. Available online: http://trc.nist.gov/TDE/TDE_Help/eos-PC-SAFT.htm (accessed on 19 June 2014).

9. Senscomp. Available online: http://www.senscomp.com/products/ (accessed on 19 June 2014).

10. Bitadze, A.; Bates, R.; Battistin, M.; Berry, S.; Bonneau, P.; Botelho-Direito, J.; DiGirolamo, B.; Godlewski, J.; Perez-Rodriguez, E.; Zwalinski, L.; et al. A combined on-line acoustic flow meter and fluorocarbon coolant mixture analyzer for the ATLAS silicon tracker. In Proceedings of the 13th International Conference on Accelerator and Large Experimental Physics Control Systems, Grenoble, France, 10-14 October 2011. Available online: http://accelconf.web. cern.ch/AccelConf/ICALEPCS2011/papers/proceedings.pdf (accessed on 24 June 2014). 
11. ETM Professional Control. ETM professional control GmbH-A Siemens Company-WinCC Open Architecture-SCADA System. 2014. Available online: http://www.etm.at/ (accessed on 19 June 2014).

12. Bates, R.; Battistin, M.; Berry, S.; Berthoud, J.; Bitadze, A.; Bonneau, P.; Botelho-Direito, J.; Bousson, N.; Boyd, G.; Bozza, G.; et al. A custom on-line ultrasonic gas mixture analyzer with simultaneous flowmetry, developed for the upgraded evaporative cooling system of the ATLAS silicon tracker. To be published in proceedings of the 2013 Conference on Advances in Nuclear Measurement Methods and their Applications, Marseille, France, 23-27 June 2013.

13. Bozza, G.; Da Riva, E. Simulation Results for the Combined Ultrasonic Flow Meter and Binary Vapour Mixture Analyser for the ATLAS Silicon Tracker; 1180058; EN/CV, CERN: Geneva, Switzerland, 2012. Available online: https://edms.cern.ch/document/1180058/3 (accessed on 24 June 2014).

14. Dopke, J. Overview of the ATLAS insertable B-Layer (IBL) project. In Proceedings of the EPS-HEP 2013 European Physical Society Conference on High Energy Physics, Stockholm, Sweden, 18-24 July 2013. Available online: http://eps-hep2013.eu/proceedings.html (accessed on 24 June 2014).

15. Bates, R.; Battistin, M.; Berry, S.; Berthoud, J.; Bitadze, A.; Bonneau, P.; Botelho-Direito, J.; Bousson, N.; Boyd, G.; Bozza, G.; et al. Development of a custom on-line ultrasonic vapour analyzer/fowmeter for the ATLAS inner detector, with application to aseous tracking and cherenkov detectors. In Proceedings of the Topical Workshop on Electronics for Particle Physics, Oxford, UK, 17-21 September 2012; JINST 2013, doi:10.1088/1748-0221/8/ 01/C01002.

16. Bates, R.; Battistin, M.; Berry, S.; Bitadze, A.; Bonneau, P.; Bousson, N.; Boyd, G.; Botelho-Direito, J.; DiGirolamo, B.; Doubek, M.; et al. Simultaneous on-line ultrasonic flowmetry and binary gas mixture analysis for the ATLAS silicon tracker cooling control system. In Proceedings of the 14th International Conference on Accelerator \& Large Experimental Physics Control Systems, San Francisco, USA, 6-11 October, 2013. Available online: http://accelconf.web.cern.ch/AccelConf/ICALEPCS2013 (accessed on 24 June 2014).

17. ATLAS. The ATLAS Collaboration. Available online: http://atlas.web.cern.ch/Atlas/ Collaboration/ (accessed on 19 June 2014). 


\title{
A Vibration-Based Strategy for Health Monitoring of Offshore Pipelines' Girth-Welds
}

\section{Pejman Razi and Farid Taheri}

\begin{abstract}
This study presents numerical simulations and experimental verification of a vibration-based damage detection technique. Health monitoring of a submerged pipe's girth-weld against an advancing notch is attempted. Piezoelectric transducers are bonded on the pipe for sensing or actuation purposes. Vibration of the pipe is excited by two means: (i) an impulsive force; (ii) using one of the piezoelectric transducers as an actuator to propagate chirp waves into the pipe. The methodology adopts the empirical mode decomposition (EMD), which processes vibration data to establish energy-based damage indices. The results obtained from both the numerical and experimental studies confirm the integrity of the approach in identifying the existence, and progression of the advancing notch. The study also discusses and compares the performance of the two vibration excitation means in damage detection.
\end{abstract}

Reprinted from Sensors. Cite as: Razi, P.; Taheri, F. A Vibration-Based Strategy for Health Monitoring of Offshore Pipelines' Girth-Welds. Sensors 2014, 14, 17174-17191.

\section{Introduction}

Offshore pipelines are susceptible to initiation of various types of defects, including corrosion, dents, and cracking/leakage, especially in their mating interfaces (e.g., girth-welds, and bolted joints). Therefore, periodic visual inspections have to be carried out by skilled divers or remote operating vehicles (ROVs) [1]. Such inspections are usually followed by more advanced examinations (e.g., automated ultrasonic technique (AUT) or eddy current method), once any suspected areas are detected.

Health monitoring of a large network of offshore pipelines, even at a preliminary stage (i.e., visual inspection), is usually a cumbersome and costly practice. Vibration-based approaches have therefore been developed and been proven to be relatively successful in detecting damage [1-4]. Therefore, they could potentially reduce the requirement of summoning skilled divers for performing the initial examinations, thus facilitating quicker and more cost-efficient inspections.

To the best knowledge of the authors, there have been very few studies conducted on damage detection of submerged structures; some of the noteworthy ones are briefly mentioned here. Na and Kundu [1] applied the guided wave technique for detection of mechanical defects (e.g., a dent, and removed material) in a scaled submerged pipe. The health monitoring of the pipe was achieved by a transmitter and a receiver, each located on either ends of the pipe. The relatively long range of inspection was noted as the advantage of the method. However, adjustment of the transmitter angle (in a trial and error fashion) was necessitated to achieve a reliable identification of damage in the submerged pipe.

Rizzo et al. [5] developed a method for health monitoring of a submerged plate hosting a notch and corrosion. A non-contact pulsed laser unit introduced stress waves into the plate. Two immersion non-contact sensors, made of piezoelectric materials, captured the reflected waves. The plate was scanned along its length by a pulse/sensing unit at discrete locations. The continuous wavelet 
transform (CWT) was used to extract a damage sensitive parameter from the time modulated frequency domain of the sensors' signals. The method could identify the location of damage by producing relatively large damage indices as the sensing probes passed across the defect regions. They marked the inclination angle along with the relative location of their adopted transducers-damage, and the proximity of the sensors as the influential parameters in the damage detection of the submerged plate.

A research team also conducted a series of extensive numerical and experimental studies to identify free-spanning (a segment of a pipe that is suspended and does not have the seabed or soil support) and corrosion along onshore and offshore pipelines [6-11]. In their study, damage detection was facilitated mainly via two approaches: (i) observation of pipeline's eigenvalues, and (ii) processing of the acceleration time-history data gathered along the pipe as the pipe underwent random or forced vibrations. The team could successfully identify the location of damage. However, there were some inconsistencies in quantifying damage size. Moreover, the free-spanning of pipelines was more reliably detected rather than the corrosion. Furthermore, in some cases, they failed to detect small corrosion; however, free-spanning of pipelines greater than $5 \%$ of the pipe's length was confidently identified.

Chen et al. [12] developed a strategy for health monitoring of a submerged plate against corrosion. They used piezoelectric transducers to generate and receive lamb waves. The received signals were processed within a probability-based diagnostic imaging approach to identify the damage. The numerical and experimental verification of the method yielded successful results in identifying the damage location.

In the present work, numerical simulations and experimental verification of a vibration-based damage detection methodology (developed in our research group [13]) for health monitoring of a submerged pipe's girth-weld against a propagating notch are presented. The performances of two vibration excitation approaches (i.e., impact and chirp excitation methods) in damage detection are compared. Additionally, the study examines the sensitivity of the method's diagnostic capability to the presence of fluid by which the pipe is pressurized. The paper begins with providing a brief introduction on the vibration-based damage detection strategy adopted in this work. This is followed by the details of the developed numerical model and the experimental setup used in the study. Finally, a discussion on the outcome of the damage detection is presented.

\section{EMD Energy Damage Index (EMD_EDI)}

Huang et al. [14] introduced a robust signal processing technique in 1998, which was referred to as the empirical mode decomposition (EMD). In brief, the EMD decomposes a time-domain signal into its oscillatory components referred to as the intrinsic mode functions (IMFs). The decomposition is accomplished through the so-called "sifting process", which is an empirical and data-driven algorithm [14]. The reader is referred to [15] for a detailed explanation on how the IMFs of a typical signal, that are recorded during a vibration event is extracted. Hilbert transform (HT) is then applied to the IMFs to unveil their time-modulated frequency components. The joint application of the EMD and the HT is called the Hilbert-Huang transform (HHT). The HHT outperforms the wavelet transform (WT), in the sense that it can accommodate both linear and non-linear systems. In addition, it is a data-driven signal processing algorithm, thus its performance would not be affected by pre-defined functions typically used in the fast Fourier transform (FFT) or WT. 
Thereafter, the EMD has been used in data interpretation in various disciplines [14]. For instance, it has been shown that the first two IMFs extracted from vibration data of a system would be sensitive to presence of damage $[13,16]$. On the same basis, Cherghai and Taheri [13] introduced the energy of the IMFs as an efficient and robust damage indicator, defined as follows:

$$
E=\int_{0}^{t_{0}}(I M F 1)^{2} d t
$$

where $t_{0}$ is the signal duration. Subsequently, damage indices can be established as follows:

$$
E M D_{-} E D I=\left|\frac{E_{\text {healthy }}-E_{\text {Damaged }}}{E_{\text {healthy }}}\right| \times 100
$$

In the above equation, $E_{\text {Healthy, }}$ and $E_{\text {Damaged }}$ are the energy terms calculated from vibration signals of individual sensors gathered at the initial state (considered as the healthy state) and the subsequent (or potentially, the damaged) state of a given structure, respectively. Sensors producing relatively higher damage indices infer presence and the location of damage. Progression of damage can also be quantified by noting the increasing value of damage indices monitored as a function of time. Several experimental case studies accompanied by numerical simulations have been conducted to verify the integrity and effectiveness of the proposed methodology [2-4,15,17]. This method, which was developed in our research group, has been therefore adopted in the current study.

\section{Finite Element Modeling}

\subsection{Modeling of a Submerged Pipe Equipped with Piezoelectric Transducers}

An aluminum pipe was considered in this case study. The material properties and dimensions of the pipe are listed in Table 1. The pipe was modeled with a total of 33,440 solid elements using ABAQUS's element C3D8R (the continuum three-dimensional eight-node reduced integration element with three translational degrees of freedom per node). Using the same element, a mid-span girth-weld was modeled for the pipe (see Figure 1). A bulge with $10 \mathrm{~mm}$ width and $2 \mathrm{~mm}$ height was used to model the girth-weld over the pipe circumference, taking into account the local stiffening introduced by the weld material. To account for a clamped-clamped boundary condition, the translational degrees of freedom of the elements at both ends of the pipe were restrained.

Table 1. Dimensions and mechanical properties of the aluminum pipe.

\begin{tabular}{cc}
\hline \multicolumn{2}{c}{ Mechanical Properties } \\
\hline Elastic modulus $(\mathrm{GPa})$ & 68.9 \\
Density $\left(\mathrm{kg} / \mathrm{m}^{3}\right)$ & 2700 \\
Poisson's ratio & 0.33 \\
\hline Dimensions $(\mathrm{m})$ & \\
\hline Length & 1 \\
Outer diameter & 0.06 \\
Wall thickness & 0.0052 \\
\hline
\end{tabular}


Figure 1. Finite element model of the submerged pipe incorporating piezoelectric sensors.

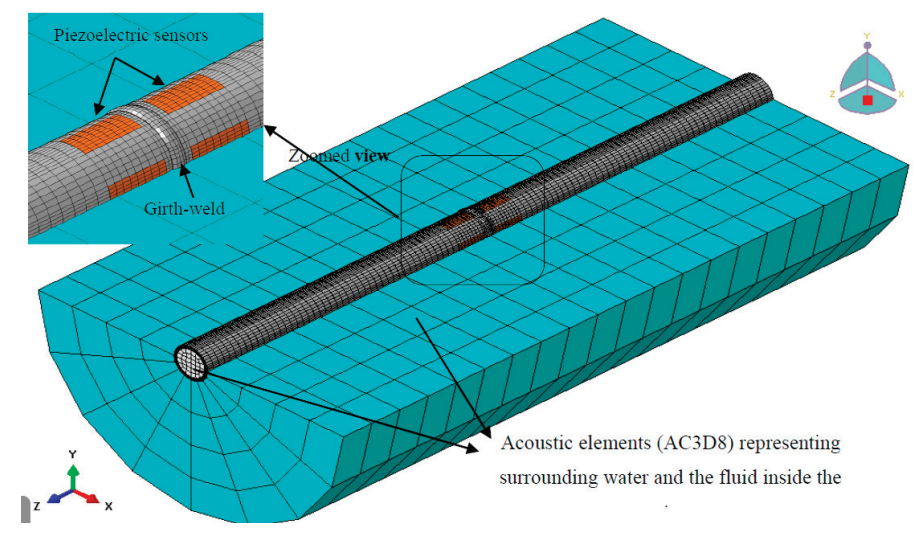

To monitor pipe's vibration, eight piezoelectric sensors were bonded to the pipe, $5 \mathrm{~mm}$ away from either sides of the girth-weld in the configuration depicted in Figure 1. The sensors' dimensions were measured to be $(45 \times 20 \times 0.15) \mathrm{mm}$. A total of 64 three-dimensional eight-node piezoelectric elements (C3D8E) were used to model each piezoelectric sensor. The "TIE" constraints were used to connect the sensors and pipe's nodes at their interfaces. The sensors were made of piezoceramics (model: PZT-5H) with the following electro-mechanical properties:

$$
\begin{gathered}
\mathrm{S}_{\mathrm{E}}\left[\frac{\mathrm{m}^{2}}{\mathrm{~N}}\right]=\left(10^{-12}\right)\left[\begin{array}{cccccc}
16.5 & -4.78 & -8.45 & 0 & 0 & 0 \\
-4.78 & 16.5 & -8.45 & 0 & 0 & 0 \\
-8.45 & -8.45 & 20.7 & 0 & 0 & 0 \\
0 & 0 & 0 & 43.5 & 0 & 0 \\
0 & 0 & 0 & 0 & 43.5 & 0 \\
0 & 0 & 0 & 0 & 0 & 42.6
\end{array}\right] \\
\mathrm{d}\left[\frac{\mathrm{C}}{\mathrm{N}}\right]=\left(10^{-12}\right)\left[\begin{array}{cccccc}
0 & 0 & 0 & 0 & 741 & 0 \\
0 & 0 & 0 & 741 & 0 & 0 \\
-274 & -274 & 593 & 0 & 0 & 0
\end{array}\right] \\
\varepsilon^{\mathrm{S}}[\mathrm{F} / \mathrm{m}]=\left(10^{-9}\right)\left[\begin{array}{ccc}
27.7 & 0 & 0 \\
0 & 27.7 & 0 \\
0 & 0 & 30.1
\end{array}\right]
\end{gathered}
$$

where $S_{E}$ is the compliance matrix, $d$ is the piezoelectric coupling matrix, and $\varepsilon^{S}$ is the permittivity of the piezoceramic. The density of the piezoceramic was taken as $7500 \mathrm{~kg} / \mathrm{m}^{3}$. Individual piezoelectric transducers could serve as a sensor and an actuator. They could turn into an actuator once a surface charge is defined for one of their surfaces.

ABAQUS's acoustic element, AC3D8 (an eight-node three-dimensional acoustic element) was used to model the surrounding water and the internal fluid in the computational domain; 1280 and 640 elements constructed the medium and the internal fluid, respectively. Density and bulk modulus values of water were taken as $997 \mathrm{~kg} / \mathrm{m}^{3}$, and $2.13 \mathrm{GPa}$, respectively, and $1.2 \mathrm{~kg} / \mathrm{m}^{3}$ and 101,000 Pa for air. The "TIE" constraints were also used to form the interfaces of the pipe and external and internal fluids. The constraints correlated the pipe's surface displacement degrees of freedom to the neighboring fluid elements' pressure degrees of freedom [18]. 
The question was raised whether one could model the pipe within an effective water depth (EWD), as opposed modeling a large volume of water, hence reducing the computational cost without sacrificing the accuracy. To establish the appropriate EWD, one can establish a boundary such that the reflected waves from that boundary would not affect the pipe's vibration. In other words, the reflection from the boundary would become negligible once the boundary is located adequately far from a vibrating structure [18].

Figure 2. A submerged pipe with: (a) radiation and (b) rigid wall boundaries.

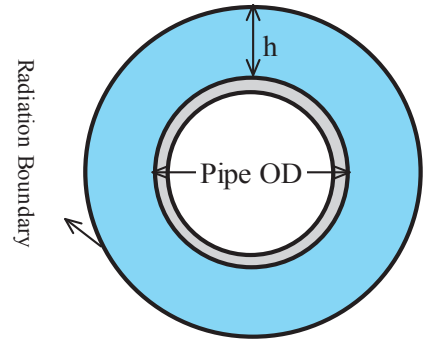

(a)

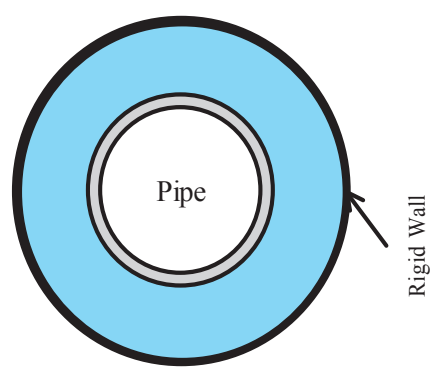

(b)

Two different boundary conditions were considered as the exterior boundaries of water in order to establish the EWD; they were (i) radiation and (ii) rigid-wall (see Figure 2). The radiation boundary would facilitate transmission of the acoustic waves across the boundaries with little reflection of energy back into the acoustic domain. On the other hand, the acoustic waves would reflect back into the acoustic domain after they hit the rigid-wall boundary [18]. Subsequently, the variation of the first-three natural frequencies of the submerged pipe against the step-wise levels of submergence, $h / O D$, was observed. $h$ is the height of water above the pipe, and $O D$ is the pipe's outer diameter.

The results shown in Figure 3 indicate that one could assume an EWD equal to four times the outer diameter of the pipe, beyond which, the boundary-type would not affect the natural frequencies of the pipe; therefore, $E W D=h / O D=4$ is considered in the analysis, hereafter.

Figure 3. Evolution of the pipe's eigenvalues: (a) first eigenvalue, (b) second eigenvalue, (c) third eigenvalue.

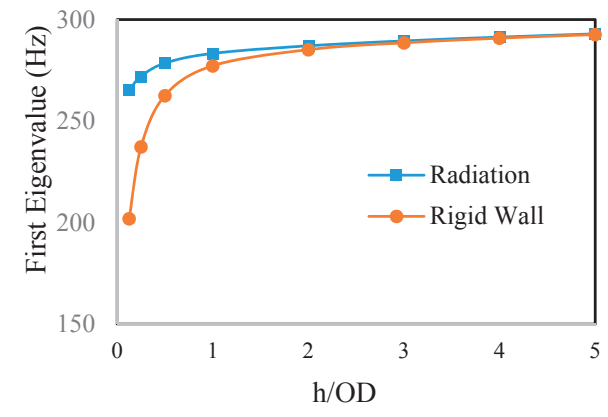

(a)

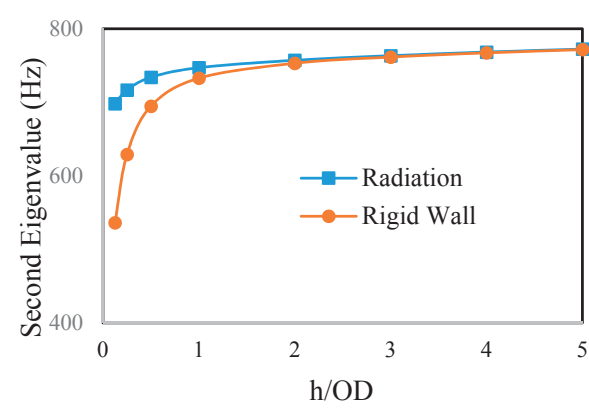

(b) 
Figure 3. Cont.

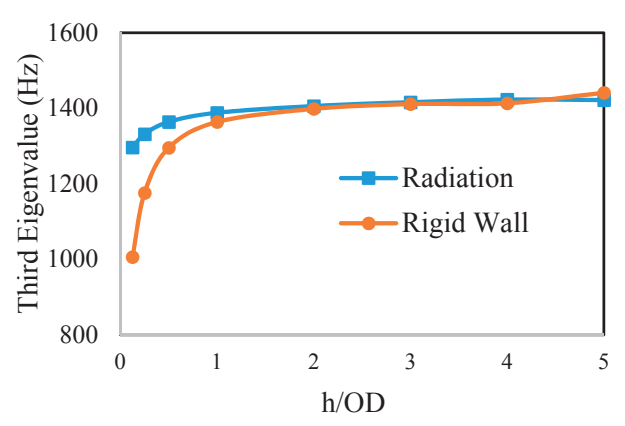

(c)

In addition, a mesh convergence study was conducted to ensure the accurate representation of the submerged pipe model. The mesh density was increased to a level such that the variation in the first-three eigenvalues remained below $1 \%$.

\subsection{Transient Analysis}

Health monitoring of the submerged pipe was accomplished by conducting a series of transient dynamic analysis solved by the implicit solution algorithm of the ABAQUS. Two excitation approaches were adopted in the damage detection trials. First, an impulse force, similar to the force that was produced by a pneumatic hammer that was used in the experiment, was prescribed to the pipe by defining a force with magnitude of $1000 \mathrm{~N}$; this force was assumed to be acting over a very short period of time $(0.0003 \mathrm{~s})$. The other alternative excitation method was achieved by using one of the piezoelectric transducers as an actuator. In this way, a chirp signal was propagated into the pipe by the actuator. The adopted chirp, defined as a surface charge in the piezoelectric actuator, contained a frequency range of $10-5000 \mathrm{~Hz}$, varied linearly over $0.05 \mathrm{~s}$. The schematic representations of the actuation units are elucidated in Figure 4.

Figure 4. Schematic representations of (a) the impact and (b) the chirp excitations.

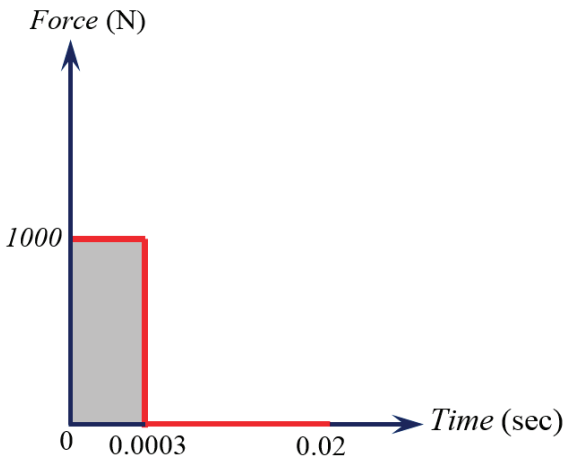

(a)

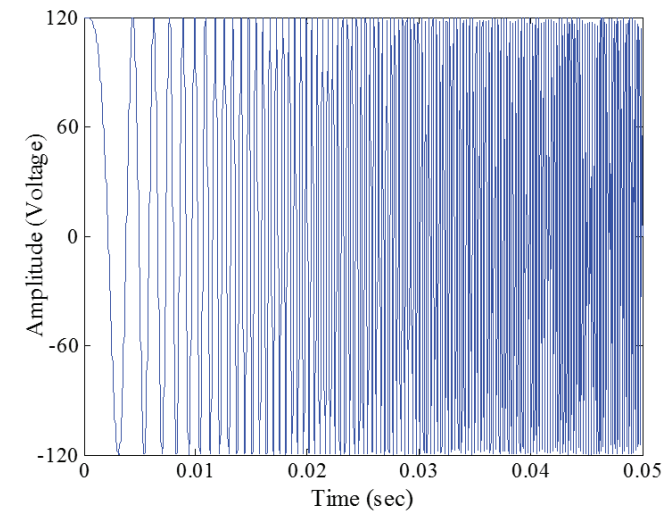

(b) 
The transient analysis was performed with an increment of $0.00002 \mathrm{~s}$. In the other words, the pipe's vibration was sampled at $50 \mathrm{kHz}(1 / 0.00002 \mathrm{~s})$, so that it could accommodate the highest excitation frequency (i.e., $5 \mathrm{kHz}$ ) through the chirp. The pipe's vibration was registered via the piezoelectric sensors' output voltage.

The analysis run-time was set to $0.02 \mathrm{~s}$ and $0.05 \mathrm{~s}$ for the tests conducted by the impact and the chirp excitation methods, respectively. It should be mentioned that a chirp signal with the same frequency content, but varying over $0.5 \mathrm{~s}$ (similar to the experimental study), was initially considered. However, a time-sensitivity analysis was performed and revealed the insensitivity of the damage detection's outcome to the selected time modulation of the chirp signal. Therefore, the aforementioned chirp was applied over $0.05 \mathrm{~s}$ in order to reduce the computational cost without sacrificing the accuracy.

\section{Experimental Framework}

An aluminum pipe with the same material properties and dimensions listed in Table 1 was considered for the experimental segment of the work. The tests were conducted in a $2.45 \times 1.12 \times 0.78$ $\mathrm{m}^{3}$ laboratory tank. The pipe was clamped at both ends as shown in Figure 5. A pressure gauge, mounted on the pipe's cap, was used to monitor the internal fluid pressure.

Eight flexible piezoelectric actuator/sensors (model: pa16n, Mide Technology Corporation, Medford, MA, USA) were bonded to the pipe in either sides of the girth-weld as shown in Figure 5b. The transducers were enclosed by a special waterproof coating; however, the electrical connections were sealed manually by applying a generous amount of silicon, and then each individual connection was further secured by heat shrink sleeves.

Figure 5. Schematic of the experimental setup for conducting the damage detection trials.
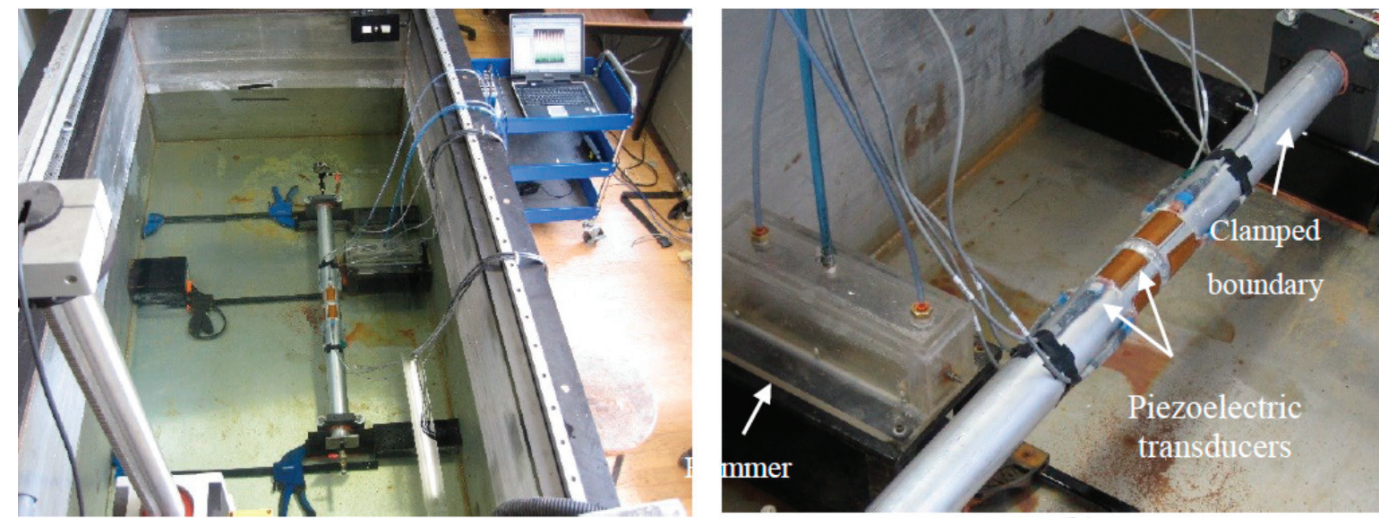
Figure 6. Schematic design of the pneumatic hammer.

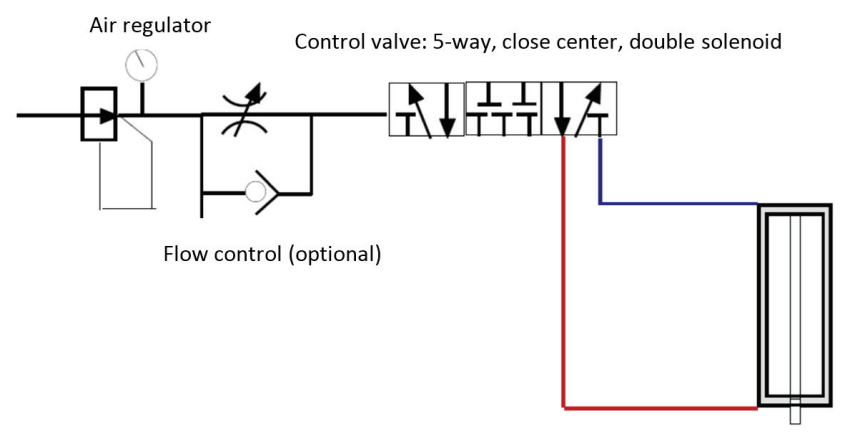

As stated earlier, two excitation methods were considered for conducting the damage detection trials:

(1) Impact

A waterproofed pneumatic hammer was designed and fabricated in-house to generate the required impulsive forces. Figure 6 shows the symbolic design of the hammer. An air regulator assured the flow of air with a constant pressure, thus ensuring the consistency of the impulse load. A flow control tuned the intensity of the impulse load. Back and forth strikes of the piston was controlled by a solenoid valve through an electrical switch. The cylinder and piston assembly was waterproofed by incorporating a Plexiglas box. An O-ring sealed the box/piston gap. A close-up of the fabricated hammer is shown in Figure 5b.

(2) Chirp waves

The second actuation means was accomplished by using one of the piezoelectric transducers as an actuator, thus propagating chirp waves in the pipe. A signal generator (model 33210A, available from Agilent Technologies, Santa Clara, CA, USA) was used to generate the chirp signals. The signals were then amplified via a power amplifier (model: 790 series available from PCB Piezotronics, Inc., Depew, New York, NY, USA), before being injected to the piezoelectric actuator. The chirp signals contained frequency range of $10-5000 \mathrm{~Hz}$, varied linearly over $0.5 \mathrm{~s}$. The piezoelectric-based excitation method provides the following main advantages over the impulsive excitation, as typically generated by an impact hammer:

i. It effectively manages the consistency of excitation, thus minimizing the discrepancy in repeated trials' measurements; the force generated by an impulse hammer is vulnerable to severe inconsistencies due to several factors, including small deviations in the impact location and the magnitude of generated force.

ii. It allows exploitation of a wide range of frequencies, and selection of various types of excitation signals (i.e., chirp, burst, and random).

The vibration signals gathered through the sensors were digitized by a data acquisition system (NI-9215 in a compact chassis manufactured by National Instrument Inc., Austin, TX, USA). The sampling rate was set to $50 \mathrm{kHz}$. 


\section{Model Verification}

An experimental study was conducted in our laboratory tank to verify the integrity of the developed numerical model before beginning the damage detection process. For that, the experimentally measured eigenvalues of a submerged pipe were compared against those obtained from the simulation. The aluminum pipe was hanged in the tank by soft elastic ropes emulating a free-free boundary condition. One of the piezoelectric transducers was used to propagate the chirp signal along the pipe. The pipe's vibration was recorded by one of the piezoelectric sensors, whose output was a voltage-signal. The experiment was simulated by the numerical model described earlier. The eigenvalues of the submerged pipe were determined by applying the FFT to the pipe's forced vibration signals obtained from the experimental study and the model. The first-three eigenvalues of the submerged pipe, obtained via the two approaches, were compared as tabulated in Table 2. The reasonable agreement between the experimental and numerical results confirms the integrity of the developed model.

Table 2. Eigenvalues of the submerged pipe.

\begin{tabular}{cccc}
\hline Eigenvalues (Hz) & Experiment & Numerical Model & Difference (\%) \\
\hline $\mathrm{f}_{1}$ & 200.0 & 198.5 & 0.7 \\
$\mathrm{f}_{2}$ & 542.8 & 533.0 & 1.8 \\
$\mathrm{f}_{3}$ & 1030.6 & 1002.5 & 2.7 \\
\hline
\end{tabular}

\section{Damage Scenarios}

Health monitoring of the pipe's girth-weld was attempted by utilizing the outlined damage detection algorithm. A notch of $1 \mathrm{~mm}$ depth (i.e., 19\% of the wall thickness) was introduced adjacent to the girth-weld and was then propagated to a depth of $4 \mathrm{~mm}$ (i.e., $77 \%$ of the wall thickness) in $1 \mathrm{~mm}$ increments. In the experiments, the notches were created by a jeweler saw. Two different conditions were considered for the pipe's internal loading conditions: (i) the pipe pressurized with air $(\mathrm{P}=1 \mathrm{MPa})$ and (ii) with water $(\mathrm{P}=5 \mathrm{MPa})$. Figure 7 depicts the notch locations and the arrangements of sensors/actuators in the four damage scenarios considered in this study. The experimental framework also incorporated the above-mentioned excitation methods for comparative purposes.

Figure 7. Schematic view of damage scenarios. The pipe was pressurized by air ((a) and (c)) and water ((b) and (d)).

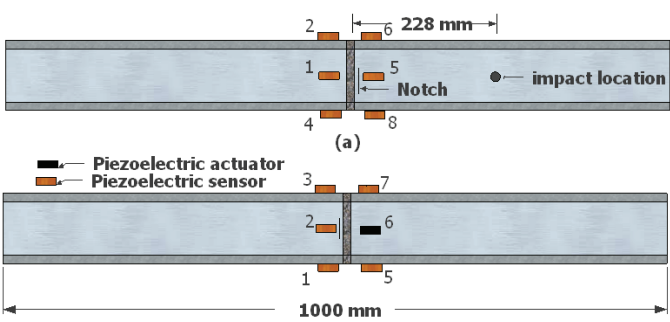

(c)

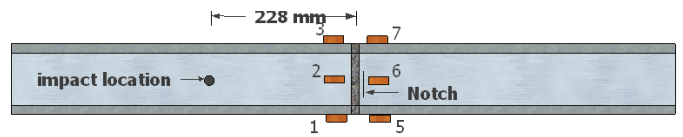

(b)

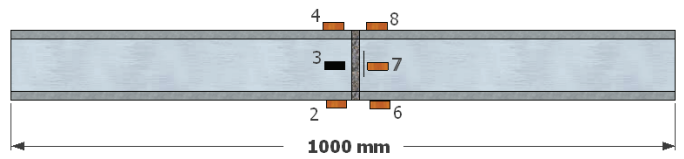

(d) 
The numerical simulations included execution of a transient analysis to capture the dynamic response of the pipe at its initial state (i.e., the healthy state), as well as for each incremental damaged state. The vibration signals at each run were recorded through the sensors. An in-house MATLAB-code was developed to include the adopted damage detection algorithm (EMD_EDI). The code performed the following tasks on the vibration data:

(i) Filtered the signals (the code applied low-pass filters of [0-1000] Hz and [0-5000] Hz to the signals obtained due to the application of the impulse load and chirp excitation, respectively.)

(ii) Extracted the IMFs of vibration signals through EMD (see Figure 8)

(iii) Calculated the first IMF's energy (using Equation (1))

(iv) Established the damage indices (using Equation (2))

Figure 8. A typical sensor's vibration response to the chirp (original signal) and its first five IMFs obtained through EMD.

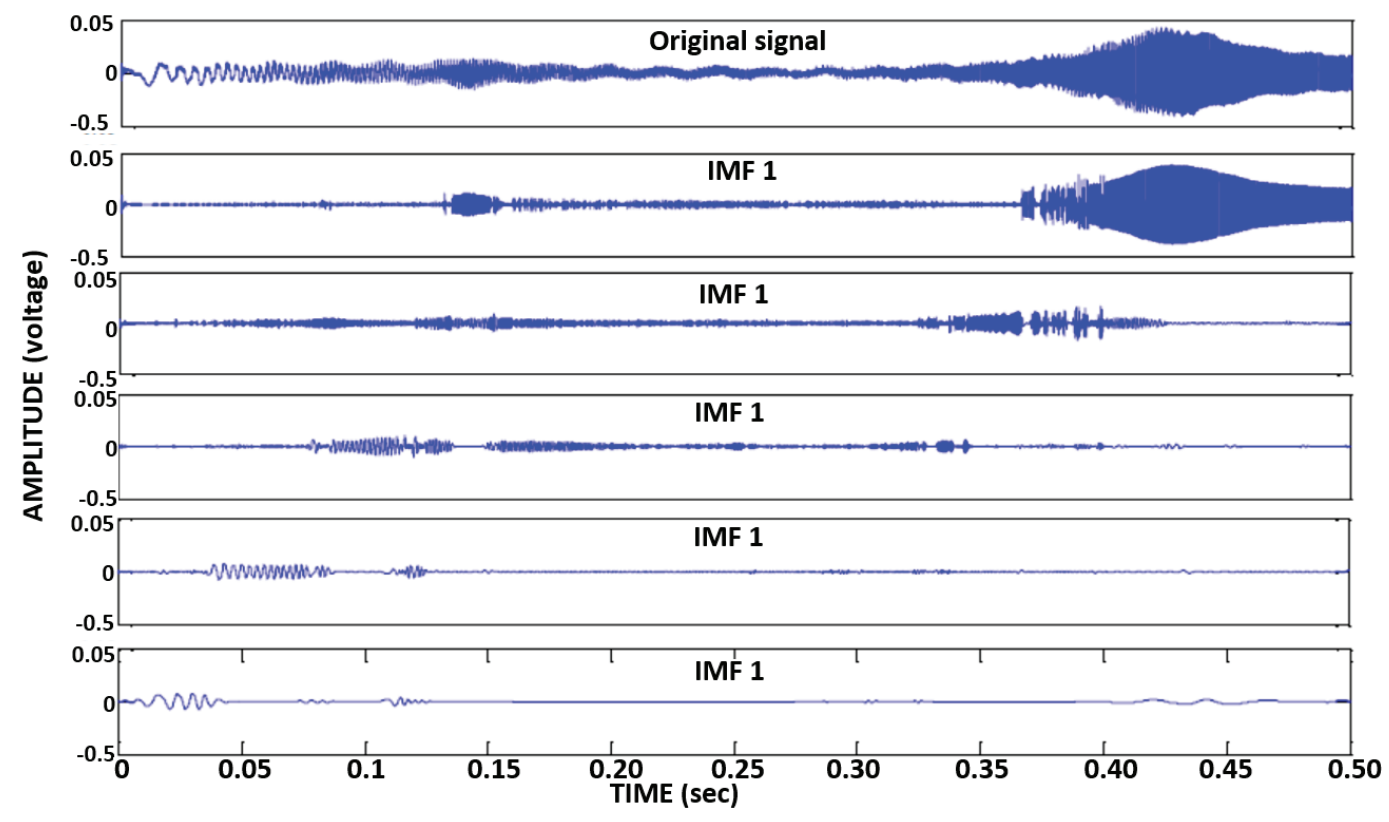

\section{Results and Discussions}

In this section, the results of the damage detection trials conducted by the two excitation technique are presented and the outcomes are discusses.

\subsection{Damage Detection Results-Impact Test}

The charts shown in Figure 9 illustrate the results of the damage detection conducted by the impact test. The results obtained from both the numerical and experimental studies indicate that at least half of the sensors could effectively detect the existence of the damage (i.e., notch), and trace 
its advancement. Half of the sensors yielded higher damage indices as the notch depth increased. The only exception was in the case shown in Figure 9d. In that case, while the existence of the notch was identified with notably large indices, its advancement, however, was underestimated at its final increment. The rest of the sensors could also discern the existence of the notch; however, they occasionally failed to predict its advancement.

It can be inferred from the results that the relative location of the sensor and actuators with respect to the notch is responsible for the variability seen in the results. Those sensors receiving their vibration waves passing through the notch outperformed the rest of the sensors in terms of damage detection accuracy. This statement can be further explained by referring to Figure $9 b$, which presents the results of the damage detection for the scenario considered in Figure $7 b$. The figure shows that signals of sensors 5 to 8 were able to trace the notch advancement, while those of sensors 1 and 3 failed to do so. In Figure 9a, EMD_EDIs of sensors 1-4 signals, which conform to the stated condition, produced noticeably higher damage indices, as well as providing a clearer indication of damage advancement.

Based on the analysis of the results, one can postulate that the diagnostic capabilities of half of the sensors were affected by the reflection of the waves from the notch. Conducting a systematic parametric study on pipes with larger diameters could shed more light on the observed behavior and validity of the noted postulation.

Figure 9. Damage indices obtained from the impact test: numerical ((a) and (b)) and experimental study $((\mathbf{c})$ and $(\mathbf{d}))$. Note that a/t is the ratio of notch's depth to pipe's wall thickness.

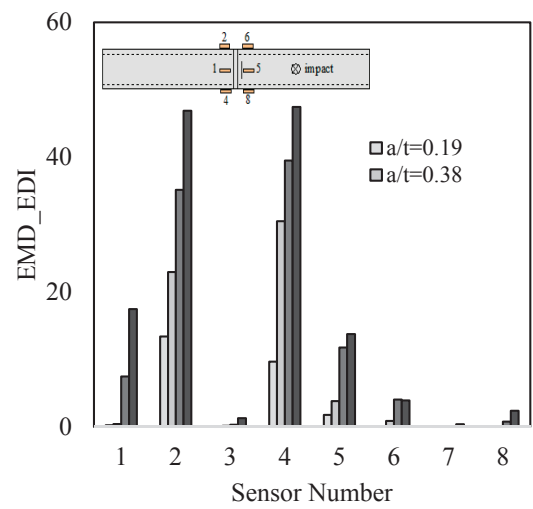

(a)

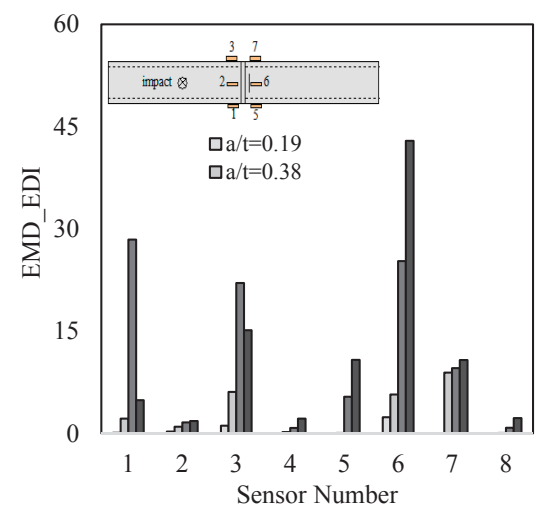

(b) 
Figure 9. Cont.

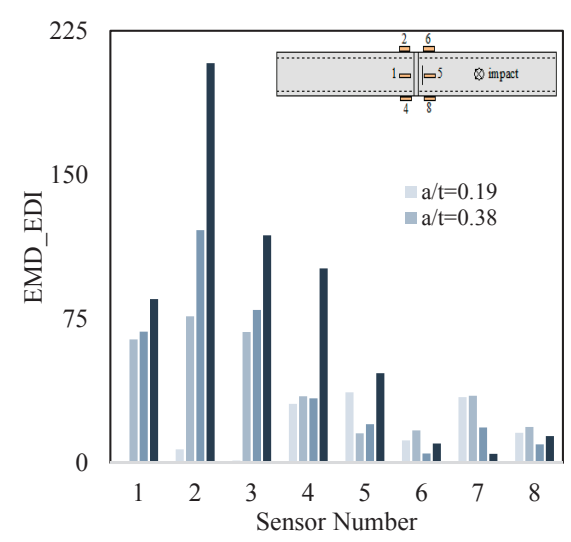

(c)

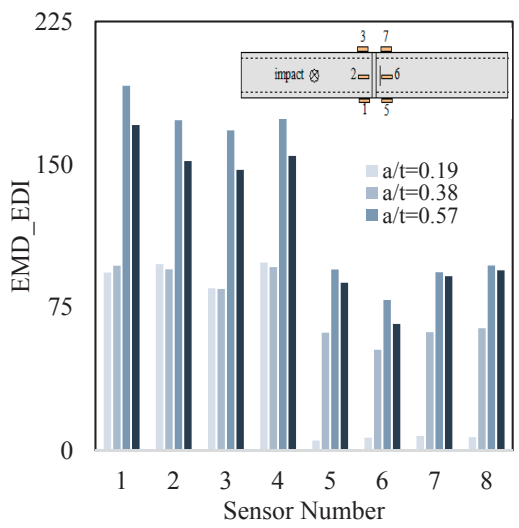

(d)

\subsection{Damage Detection Results-Chirp Test}

The results of the damage detection tried by the chirp method qualitatively confirm the findings of our previous trial, as noted above (i.e., when the pipe was excited by an impact). Comparatively, the advancement of the notch could be traced more accurately when the chirp method is used to excite the pipes.

Figure 10. Damage indices obtained from the chirp test: numerical ((a) and (b)) and experimental study $((\mathbf{c})$ and $(\mathbf{d}))$. Note that a/t is the ratio of notch's depth to pipe's wall thickness.

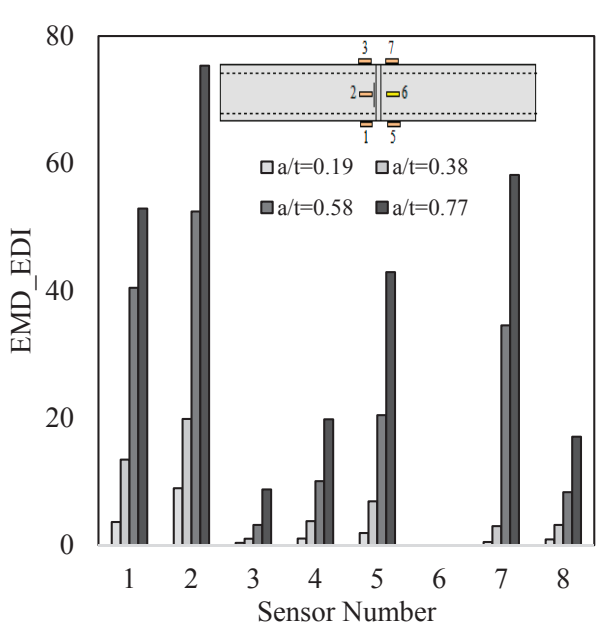

(a)

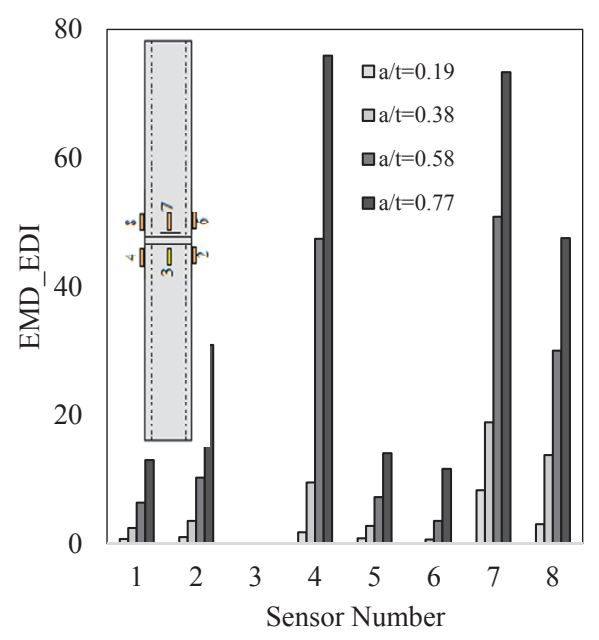

(b) 
Figure 10. Cont.

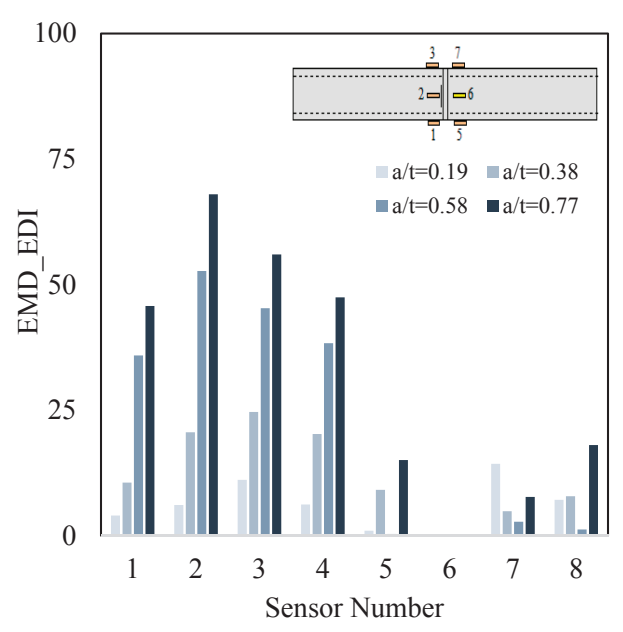

(c)

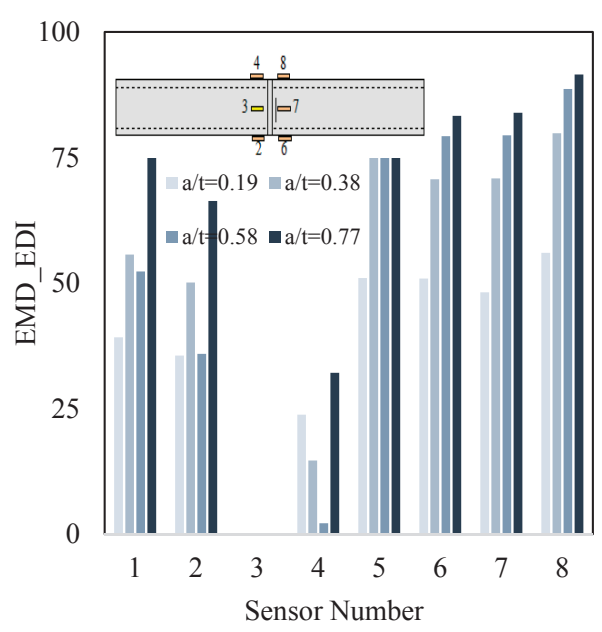

(d)

\subsection{Comparison of the Application of Impact and Chirp}

The diagnostic capability of the method in damage localization was also investigated under the both excitations approaches. The numerical results illustrated in Figure 9a,b, and Figure 10a and b suggest that the closest sensor to damage produced the highest damage index; hence, it can be concluded that an accurate damage localization could also be produced by the presented methodology. In consideration of the results, the only exception applies to the first damage scenario (see Figure 9a). In that situation, the closest sensor to the damage (i.e., sensor 5) did not produce the largest EMD_EDI. This is because, it was located in-between the source of excitation and the notch, thus a low diagnostic capability was already anticipated based on the observations explained in Section 7.1.

In comparison to the numerical results, the EDIs obtained by processing of the experimental data produced somewhat controversial results in terms of damage localization. The results presented in Figure 10c show that signals collected through sensor 2 (the closest sensor to the damage) yielded the largest EMD_EDI, hence identifying the exact location of the notch on pipe's circumference. For the remaining damage scenarios, the location of damage could not be discerned successfully. It is believed that the inevitable uncertainties involved in the experiments (e.g., the variable pipe/sensor's bond-strength, and the minute inequalities in sensors/girth-weld distances, which occurred unintentionally during the process of bonding the sensors to the pipe) could have affected the accuracy of damage localization.

Taking the above observations into account, it could be suggested that one requires a minimum of two piezoelectric sensors for accurate health monitoring of such damage conditions (such that one sensor is bonded on each side of the girth-weld, opposite to one another with respect to the axial direction). A more effective health monitoring, however, could be achieved by conducting two separate trials, using either of the excitation techniques. In the case of vibration excitation by an impulse force, the two tests would consist of impacting the pipe at both sides of the girth-weld, 
and recording the associated vibration of each impact. In the case of chirping, the sensors can alternate their tasks in the form of a sensor and actuator, respectively. In this way, upon completion of the two tests, the existence and severity of damage could be more effectively discerned by noting the largest EMD_EDI obtained from the two trials. The conclusion holds also true for the numerical model, so long as the chirp excitation method is used as the means of excitation; otherwise, at least four sensors (two placed on each side of the girth-weld) are required for a successful damage detection, since it was observed that some of the sensors could not sense the existence of the damage (see Figure 9a,b).

In all, both the numerical and experimental studies provided satisfactory results with respect to identification of an advancing notch, regardless of the fluid-type the pipe carried (i.e., compressible (air) or incompressible (water)). It can therefore be concluded that the variable damping introduced by the presence of fluid inside the pipe would not weaken the method's diagnostic capability.

Moreover, the chirp excitation method generally outperformed the commonly-used impulsive force excitation technique, in terms of both damage localization and prediction of its advancement. In addition, the results obtained through the sensors in the experimental studies managed to detect the onset and advancement of damage with larger EMD_EDIs compared to the EMD_EDIs obtained through the numerical simulations. As for the noted discrepancies between the results of the experimental and numerical investigations utilizing the impact hammer, the reason could be due to the difference in the actual time-domain history of the applied impulse forces. It should be noted that since there was no direct means to measure the magnitude of the force during the tests, therefore, an approximate loading amplitude (based on our previously conducted trials and experience with a modal hammer) was selected for replicating the impulse force in the numerical study. Furthermore, due to unavoidable circumstance, the pneumatic hammer produced double-impact at each loading application. As a result, the time resolution of the repeated force could not be retrieved accurately within each test. Therefore, it was idealized as a single impulse force in the numerical simulations.

\section{Remarks on the Environmental/Operational Conditions}

In the experimental investigation, the repeatability of the measurements was established by comparing the energy terms resulting from the applied excitation from one trial to the next for total of ten trials. The consistency of the impact force was determined to be $92 \%$ and $98 \%$ for the pneumatic hammer and piezoelectric actuator, respectively. The deviations in the measurements are believed to be due the inevitable noise associated with the instruments and laboratory noise, as well as due to minor inconsistencies produced during the hammer impacts.

It was also of interest to examine the simultaneous effects of the noise and disturbance associated to the pipe's constraints (which could occur in real applications) on the repeatability of the measurements. For that purpose, the clamping torque of the bolts securing the collar on one of the pipe's ends tested in air (see Figure 5) was reduced from (40 to 20) $\mathrm{N} \cdot \mathrm{m}$ in increments of $5 \mathrm{~N} \cdot \mathrm{m}$. The precise measurement of the clamping forces on the bolted assembly was facilitated using a digital torque meter. A chirp-type wave was propagated via piezoelectric sensor 5 at each individual torque level, and the vibration response was recorded by the first four sensors (sensor 1-4). 
The energy of each sensor's vibration signal at the specified clamping forces was calculated according to Equation (1), and was subsequently normalized with respect to the energy corresponding to the maximum torque level (i.e., $40 \mathrm{~N} \cdot \mathrm{m}$ ). Figure 11 reports the average of ten measurements at the specified torque levels. As can be seen, the maximum variation in the calculated energies is only 6\%. One can therefore make an implicit conclusion that EMD_EDIs with values above 6\% would indicate the existence of a damage within a given trial. EMD_EDIs below this threshold could not be attributed to the presence of damage with confidence, since they might be produced as a result of a disturbance in the boundary conditions.

Figure 11. The normalized energy as a function of the clamp torque level.

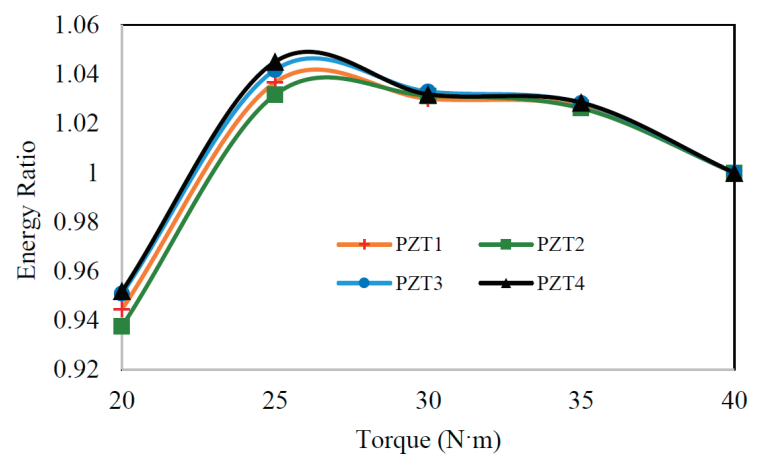

Moreover, owing to the damping of the propagated waves, it is anticipated that the observed variations would be smaller for cases where the supports are located farther from the inspection zone.

Operational variability such as abrupt changes in the internal pressure of the pipe could also affect the integrity of a vibration-based damage detection trial. The additional stiffening generated by the internal pressure can variably modify the overall stiffness of the pipe, thereby its vibration response. As a result, erroneous energies, causing false alarms, could be developed. To investigate the criticality of this issue, the sensitivity of the proposed energy index to such operational variability was examined. For that, different levels of pressures (i.e., 0-5 MPa) were assigned to the internal surface of the healthy pipe. At each pressure level then, the pipe was excited by an impulse load and a chirp wave according to the configuration elucidated in Figure $7 \mathrm{~b}$ and $\mathrm{d}$, respectfully, and pipe's vibration was recorded by the sensors. The energy of the vibration signal obtained through sensor 5 was calculated according to Equation (1). The energy terms were then normalized with respect to the energy of the sensor 5's vibration signal when the pipe experienced the highest pressure (i.e., $5 \mathrm{MPa})$. 
Figure 12. The normalized energy of the vibration signal as a function of internal pressure.

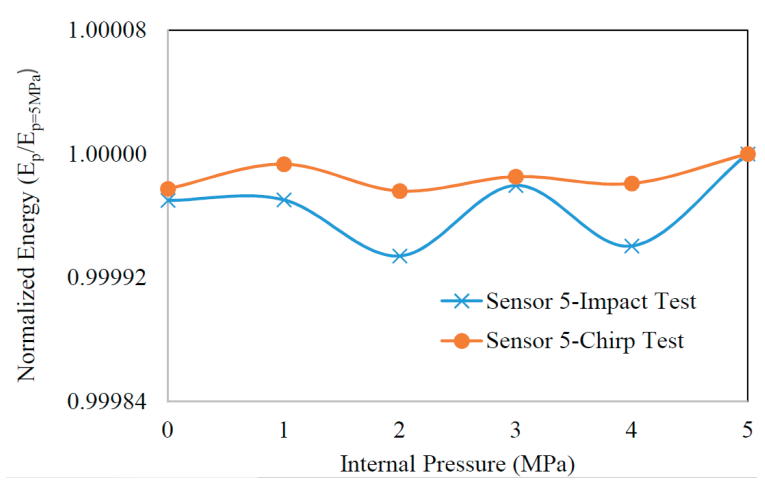

Figure 12 shows the fluctuations of the energy as a function of the applied internal pressure. It can be seen that the rate of variations in the energy was limited to below $0.1 \%$. Therefore, the study indicated that the pipe vibration remained almost unaffected under the imposed operational variability. As such, the proposed vibration-based damage detection algorithm can be reliably applied to the pipe in the presence of such variations.

\section{Conclusions}

Numerical simulations and experimental verifications of a vibration-based damage detection strategy for health monitoring of submerged pipelines' girth-welds were presented. Piezoelectric transducers were used in the capacity of actuators and sensors to excite and record pipe's vibration, respectively. The damage detection methodology incorporated the empirical mode decomposition (EMD) to process the recorded vibration signals and establish the energy-based damage indices (EMD_EDIs). The integrity and efficiency of the technique were evaluated by detection of an advancing notch that was formed in the girth-weld of two mating aluminum pipes. The results of the numerical study were compared against those obtained from the experimental investigations. Reasonable agreement was obtained between the damage detection indices produced by the numerical and experimental case studies. Examination of the EMD_EDIs produced through sensors' data revealed encouraging evidence with respect to detection of the presence and advancement of damage, regardless of the type of fluid used to pressurize the pipe.

The investigation also examined the performance of two vibration excitation approaches, namely: the impact method and chirp excitation method. The results demonstrated the effectiveness of the chirp excitation method over the impact method for damage detection of the submerged pipes. It was also revealed that method could sustain its integrity and reliability in the presence of some common environmental and operational variability (e.g., noise, disturbance in boundary conditions, and abrupt changes in the internal pressure). Finally, it was concluded that health monitoring of the submerged pipe's girth-weld could be efficiently accomplished with a minimum of two transducers, one acting as a sensor and the other as an actuator, bonded on each side of the girth-weld. 


\section{Acknowledgments}

The financial support of the Petroleum Research Atlantic Canada (PRAC) and the Natural Sciences and Engineering Council of Canada (NSERC) in support of this work is gratefully acknowledged.

\section{Author Contributions}

Pejman Razi, essentially conducted all the experiments, and most of the analysis. Farid Taheri provided Pejman Razi with the original idea and what needs to be accomplished. Pejman Razi wrote the draft manuscript, and Farid Taheri modified it.

\section{Conflicts of Interest}

The authors declare no conflict of interest.

\section{References}

1. Na, W.-B.; Kundu, T. Underwater pipeline inspection using guided waves. J. Pressure Vessel Technol. 2002, 124, 196-200.

2. Rezaei, D.; Taheri, F. Experimental validation of a novel structural damage detection method based on empirical mode decomposition. Smart Mater. Struct. 2009, doi:10.1088/0964-1726/ 18/4/045004.

3. Rezaei, D.; Taheri, F. Health monitoring of pipeline girth weld using empirical mode decomposition. Smart Mater. Struct. 2010, doi:10.1088/0964-1726/19/5/055016.

4. Razi, P.; Esmaeel, R.A.; Taheri, F. Improvement of a vibration-based damage detection approach for health monitoring of bolted flange joints in pipelines. Struct. Health Monit. 2013, 12, 207-224.

5. Rizzo, P.; Han, J.-G.; Ni, X.-L. Structural health monitoring of immersed structures by means of guided ultrasonic waves. J. Intell. Mater. Syst. Struct. 2010, 21, 1397-1407.

6. Wang, Y.; Khoo, S.; Li, A.-J.; Hao, H. FEM calibrated ARMAX model updating method for time domain damage identification. Adv. Struct. Eng. 2013, 16, 51-60.

7. Wang, Y.; Hao, H. Damage identification scheme based on compressive sensing. J. Comput. Civ. Eng. 2013, doi:10.1061/(ASCE)CP.1943-5487.0000324.

8. Bao, C.; Hao, H.; Li, Z. Vibration-based structural health monitoring of offshore pipelines, numerical and experimental study. Struct. Control Health Monit. 2013, 20, 769-788.

9. Peng, X.-L.; Hao, H. A numerical study of damage detection of underwater pipeline using vibration-based method. Int. J. Str. Stab. Dyn. 2012, doi:10.1142/S0219455412500216.

10. Peng, X.-L.; Hao, H.; Li, Z.-X. Application of wavelet packet transform in subsea pipeline bedding condition assessment. Eng. Struct. 2012, 39, 50-65.

11. Zhu, X.; Hao, H.; Peng, X. Dynamic assessment of underwater pipeline systems using statistical model updating. Int. J. Str. Stab. Dyn. 2008, 8, 271-297. 
12. Chen, J.; Su, Z.; Cheng, L. Identification of corrosion damage in submerged structures using fundamental anti-symmetric Lamb waves. Smart Mater. Struct. 2010, doi:10.1088/0964-1726/ 19/1/015004.

13. Cheraghi, N.; Taheri, F. A damage index for structural health monitoring based on the empirical mode decomposition. J. Mech. Mater. Struct. 2007, 2, 43-62.

14. Huang, N.E.; Shen, Z.; Long, S.R.; Wu, M.C.; Shih, H.H.; Zheng, Q.; Yen, N.-C.; Tung, C.C.; Liu, H.H. The empirical mode decomposition and the Hilbert spectrum for nonlinear and non-stationary time series analysis. Proc. R. Soc. Lond. A Math. Phys. Eng. Sci. 1998, 454, 903-995.

15. Razi, P.; Esmaeel, R.A.; Taheri, F. Application of a robust vibration-based non-destructive method for detection of fatigue cracks in structures. Smart Mater. Struct. 2011, doi:10.1088/ 0964-1726/20/11/115017.

16. Loutridis, S.J. Damage detection in gear systems using empirical mode decomposition. Eng. Struct. 2004, 26, 1833-1841.

17. Rezaei, D.; Taheri, F. Damage identification in beams using empirical mode decomposition. Struct. Health Monit. 2011, 10, 261-274.

18. Corporation, D.S.A. ABAQUS 2011 Version 6.10 User Manual. Available online: http://www.abaqus.com (accessed on 1 December 2011). 


\title{
Development of a Piezoelectric Vacuum Sensing Component for a Wide Pressure Range
}

\author{
Bing-Yu Wang, Fan-Chun Hsieh, Che-Yu Lin, Shao-En Chen, Fong-Zhi Chen and \\ Chia-Che Wu
}

\begin{abstract}
In this study, we develop a clamped-clamped beam-type piezoelectric vacuum pressure sensing element. The clamped-clamped piezoelectric beam is composed of a PZT layer and a copper substrate. A pair of electrodes is set near each end. An input voltage is applied to a pair of electrodes to vibrate the piezoelectric beam, and the output voltage is measured at the other pair. Because the viscous forces on the piezoelectric beam vary at different air pressures, the vibration of the beam depends on the vacuum pressure. The developed pressure sensor can sense a wide range of pressure, from $6.5 \times 10^{-6}$ to 760 Torr. The experimental results showed that the output voltage is inversely proportional to the gas damping ratio, and thus, the vacuum pressure was estimated from the output voltage.
\end{abstract}

Reprinted from Sensors. Cite as: Wang, B.-Y.; Hsieh, F.-C.; Lin, C.-Y.; Chen, S.-E.; Chen, F.-Z.; Wu, C.-C. Development of a Piezoelectric Vacuum Sensing Component for a Wide Pressure Range. Sensors 2014, 14, 22099-22112.

\section{Introduction}

Recently, vacuum technology has occupied a key position in diverse fields of advanced science and technology such as surface science, thin film technology, space science, high-energy particle accelerators, microelectronics, and materials science. In addition, vacuum technology has an increasingly wide range of industrial production applications such as product packaging, vacuum casting, vacuum drying, chemical vapor deposition, evaporation, sputtering and dry etching. The vacuum pressure ranges from atmospheric pressure (760 Torr) to ultra-high vacuum pressure ( $10^{-13}$ Torr). For example, evaporation [1] must be conducted in vacuum pressures from $10^{-7}$ to $10^{-5}$ Torr to increase the evaporation rate and to maintain the purity and density of the film. Sputtering [2] must be conducted in vacuum pressure less than $10^{-2}$ Torr to have deposited thin films which are uniformly distributed on the substrate. The low pressure environment leads to reduction in the frequency of collision of ions with gas molecules, thus increasing the mean free path of the particles. In reactive ion etching [3], the etching operation is carried out at vacuum pressures below $10^{-2}$ Torr to increase directional etching. The low pressure environment leads to reduce the probability of collision of ions and neutral particles. In the electron cyclotron resonance plasma process [4], the operating pressure is controlled at below $10^{-4}$ Torr to achieve high density and uniformity. High density plasma [5] is operated in vacuum pressures from $10^{-6}$ to $10^{-2}$ Torr, which improves the etching rate and enhances the etching direction.

Vacuum covers a wide range of pressures. The mean free path of residual gas molecules is an important parameter that defines the vacuum state, which indicates the average distance travelled by molecules between collisions with each other. In initial stages of evacuation, i.e., at low 
vacuum, the motion of gas is similar to gas flow. In high vacuum, i.e., as the vacuum pressure is lowered below $10^{-3}$ Torr, the mean free path of residual gas molecules increases, and the motion of gas gradually becomes a molecular motion. For convenience, when discussing vacuum technology, we use different gas motions to distinguish between different vacuum states.

To achieve the vacuum condition, a mechanical pump is initially used to exhaust the gas at atmospheric pressure. This initial state of gas flow is called viscous flow [6] or continuous flow. In this state, the features of gas flow are the mutual collisions between each gas molecule, movement of each gas molecule limited by surrounding molecules, friction between the gas molecules, direction of gas flow, and gas molecules moving in the same direction. As the vacuum system continues the pumping action, the gas pressure continues to decline and the gas flow state becomes transition flow [7]. This gas flow state is very complicated which part of the gas flow maintains the viscous flow state but part of them converts into the molecular flow state. When the gas pressure of the vacuum system is reduced to a certain level, the gas flow state reaches the molecular flow range [8]. In this state, gas molecules have free random motion. The collisions are elastic and consistent with the conservation of kinetic energy and momentum conservation law. The probability of a gas molecule colliding with the chamber wall is greater than the probability of it colliding with another gas molecule. Regardless of how low the pressure is, the flow state maintains molecular flow once the flow conditions in the vacuum system reach the molecular flow range.

According to the operating principle, the vacuum gauges can be distinguished as absolute vacuum gauges and relative vacuum gauges. The operating principle of an absolute vacuum gauge involves the direct measurement of the forces on the unit area. The measurement principle of a relative vacuum gauge involves the use of the relationship between gas pressure and certain physical quantities. For example, the pressure can be obtained by thermal conduction [9-14]. Moreover, the pressure also can be measured by gas-molecule ionization technology indirectly [15-17].

Usually, we use different types of vacuum gauges to measure the vacuum pressure within the respective pressure ranges from $10^{-7}$ to 760 Torr. The vacuum gauges used in low vacuum (1-760 Torr) are based on an elastic element [18-22]. The vacuum gauges used in medium vacuum $\left(10^{-3}-1\right.$ Torr $)$ are based on a thermal conduction element [10-14]. The vacuum gauges used in high vacuum $\left(10^{-7}-10^{-3}\right.$ Torr) are based on a gas ionization element [15-17].

The abovementioned gauges include the diaphragm gauge [18-22], thermal conductivity vacuum gauge, ion vacuum gauge, and viscosity vacuum gauge [23]. The operating principle of the diaphragm gauge involves the measurement of the capacitance change caused by deformation of a film surface by pressure. The pressure value can be estimated based on the capacitance change. The operating principle of the thermal conductivity vacuum gauge involves the use of heat transfer from the objects heated by gas collision. Heat conduction is proportional to the frequency of gas molecule collisions with the objects. Therefore, the vacuum pressure is proportional to the heat conduction, enabling the estimation of pressure. The operating principle of the ion vacuum gauge involves the measurement of the number of molecules in the vacuum system to determine the pressure. The operating principle of the viscosity vacuum gauge involves the use of the viscosity characteristics of residual gas in the vacuum system to determine the pressure. The spinning rotor 
viscosity vacuum gauge $[24,25]$ can be used to measure vacuum pressure ranges between $10^{-1}$ and $10^{-7}$ Torr. Gas viscosity caused by gas resistance is proportional to the degree of vacuum.

Based on existing literature, there are just few vacuum gauges that can measure the entire pressure range from $10^{-7}$ to $10^{-2}$ Torr. Current spinning rotor viscosity vacuum gauges can measure vacuum pressure ranges of $10^{-7}-10^{-1}$ Torr. Due to the presence of several components such as the ball and control coil, spinning rotor viscosity vacuum gauges increases the volume, weight, and complexity of the system, thus limiting their applications. Mortet et al. [26] used a commercially available piezoelectric bimorph cantilever as pressure sensor which detected the change in the resonance frequencies due to the drag force of the surrounding gas. Sumali et al. [25] used a bulk piezoelectric transducer shook the whole chip on which an atomic force microscope probe mounted. A laser Doppler vibrometer (LDV) with a microscope measured the velocities at a point on the chip, and 42 points along the edges and tip of the cantilever. They stressed that the air damping is proportional to pressure in the rarefied regime. Wang et al. [27] developed a micro-cantilever beam deflected using electrostatic force. They measured the capacitance between two electrodes which were mounted around the proof mass of the trapezoidal micro-cantilever beam and a sensing electrode was placed on top of the proof mass with the deflection electrode mounted beneath to determine the free decay rate of the sensing beam with respect to deflection force and vacuum pressure. However, those devices still need additional actuators to drive the element and outside sensing devices to convert deflection of beams to electrical signals. Outside sensing devices make the measurement system complex.

In this study, we designed a self-actuated and self-sensing piezoelectric pressure sensor. The piezoelectric sensors are in the form of clamped-clamped beams. The sensor was designed using two piezoelectric elements: for self-actuating and self-sensing. Applying voltage to the PZT self-actuating element causes deformation of the clamped-clamped beams. At the other end of the beam, the PZT self-sensing element produces a voltage caused by the bending of the beam. Under different vacuum pressure values, the gas viscosity and the damping ratio of devices are different. This causes different swing amplitudes and resonant frequencies of the device and results to different output voltages and resonant frequencies from the sensing element. Thus, the vacuum pressure can be calibrated. The advantages of developed vacuum sensor are self-actuating and self-sensing without additional actuators and outside sensing elements. The wide range vacuum pressure from $6.5 \times 10^{-6}$ to 760 Torr can be directly derived from piezoelectric output. Fabrication of developed vacuum sensors was easy because of simple structure.

This study has three specific goals. First, a piezoelectric pressure sensing element was developed that can be used to measure a wide range of vacuum pressure from $6.5 \times 10^{-6}$ to 760 Torr. Second, the size of sensing element was $20 \mathrm{~mm}$ length, $5 \mathrm{~mm}$ width and $200 \mu \mathrm{m}$ thick. Compared to commercial pressure sensors, the piezoelectric pressure sensors have the following advantages such as small size, low weight and simple instrumentation. Finally, the sensing elements were used to measure the pressure in nitrogen and argon to study the relationship between vacuum pressures and damping ratios of different gases. 


\section{Principle of Operation and Component Size}

\subsection{Principle of Operation}

This study proposes the clamped-clamped beam-type piezoelectric vacuum pressure sensing element, a self-actuating and self-sensing microresonator, to detect the damping ratio of the gas, thus enabling the calculation of the pressure of vacuum system. The schematic diagram of the vacuum pressure sensing element is shown in Figure 1. The sensing element comprises a PZT layer, a substrate, and two pairs of electrodes. The electrodes are placed near both ends for piezoelectric actuation and sensing. When the sinusoidal voltage signal is applied to a pair of electrodes, due to the inverse piezoelectric effect, the clamped-clamped beam vibrates and resonates. Simultaneously, the other pair of electrodes captures the vibration energy and converts it to electric energy using the positive piezoelectric effect. Finally, we measured the output voltages which varied under different gases viscosity and vacuum pressures.

Figure 1. Schematic diagram of the piezoelectric vacuum pressure sensing component.

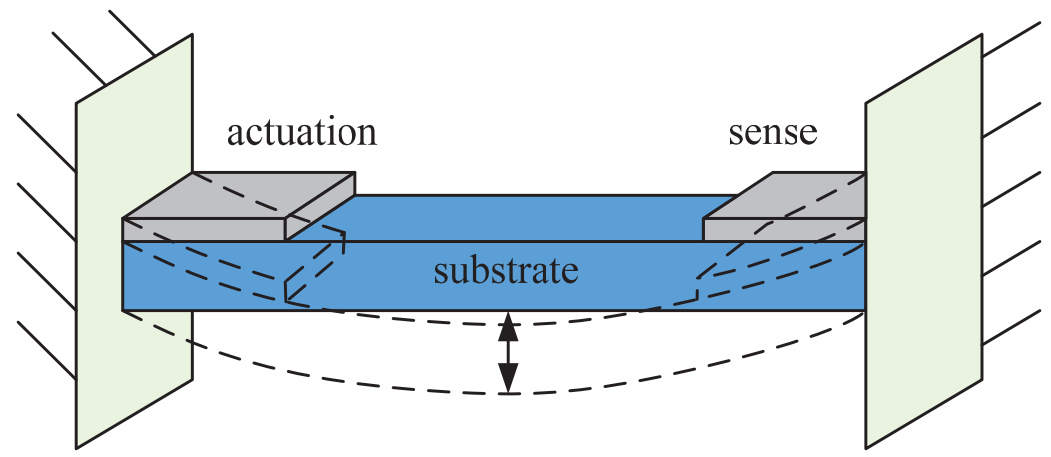

\subsection{Choice of Component Materials}

The piezoelectric sensor consists of a piezoelectric layer and a substrate. We choose PZT-5A as the piezoelectric layer and copper as the substrate. PZT-5A has a high piezoelectric constant and electromechanical coupling constant, and the energy consumption is small for conversion between mechanical energy and electrical energy. Copper has a low Young's modulus and high electrical conductivity and can reduce the operating frequency. On the other hand, it can facilitate current conduction.

\subsection{Component Size Design}

The Euler-Bernoulli beam theory is the basis of assumptions to establish the mathematical model and is used to determine the size of the vacuum pressure sensing element. The force conservative equation is given as follows: 


$$
\begin{gathered}
Y I \frac{\partial^{4} w(x, t)}{\partial x^{4}}+c_{S} I \frac{\partial^{5} w(x, t)}{\partial x^{4} \partial t}+c_{a} \frac{\partial w(x, t)}{\partial t}+m \frac{\partial^{2} w(x, t)}{\partial t^{2}}+\alpha v_{\text {in }}(t)\left[\frac{d \delta(x)}{d x}-\frac{d \delta\left(x-x_{1}\right)}{d x}\right] \\
+\alpha v_{\text {out }}(t)\left[\frac{d \delta\left(x-x_{2}\right)}{d x}-\frac{d \delta(x-L)}{d x}\right]=0
\end{gathered}
$$

where $Y I$ is the bending stiffness of the composite beam, indicating the resistance of the bending moment; $w(x, t)$ is the cantilever deflection function, which is the neutral axis of the lateral displacement of each section (y-direction is positive); $c_{s} I$ is the equivalent damping term due to the viscosity of the combination cross section, where $c_{s}$ is the strain damping coefficient and $I$ is the second moment of inertia for the combination of the cross section between the piezoelectric layer and substrate; $c_{a}$ is the air damping coefficient; $m$ is the mass per unit length; and $\alpha$ is the piezoelectric coupling term. $v_{\text {in }}(t)$ and $v_{\text {out }}(t)$ are the input and output voltages, respectively. This cantilever piezoelectric sensing element contains two pairs of electrodes. The position of the input electrode ranges from $x=0$ to $x=x_{1}$, and that of the output electrode ranges from $x=x_{2}$ to $x=L$. Only the electric field is generated in the electrode coverage.

We assume that the operation of the piezoelectric sensor considers only the influence of resonance frequency (first mode). Therefore, the ratio of the output voltage to the input voltage from the mathematical model can be written as follows [28]:

$$
\left|\frac{v_{o u t}}{v_{\text {in }}}\right|=\frac{\left|\omega \tau_{c} \lambda_{1} x_{1}\right|}{\sqrt{\left[-2 \xi \tau_{c} \omega^{3}\right]^{2}+\left[2 \xi \omega^{3}+\tau_{c} \lambda_{1} x_{2} \omega\right]^{2}}}
$$

where $\omega$ is the first resonance frequency of the cantilever, $\tau_{\mathrm{c}}$ is the time constant of the output circuit, $\lambda_{1}$ is the integrating factor, and $\xi$ is the damping term in the modal coordinate functions. This damping term can be shown as Equation (3), and it combines the effect of air damping and structural damping:

$$
\xi=\frac{c_{s} I \omega}{2 Y I}+\frac{c_{a}}{2 m \omega}
$$

To reduce the sensor size in practical applications, the cantilever length and breadth are set as 20 and $5 \mathrm{~mm}$, respectively, and the piezoelectric sheet thickness is set as $200 \mu \mathrm{m}$.

\subsection{Electrode Design of Sensor}

The two pairs of electrodes are distributed on the upper and lower surfaces of the piezoelectric material layer, which are placed on both ends of the cantilever. One pair of electrodes is placed beside the fixed boundary in the cantilever as an actuator to drive the cantilever and generate resonance. The other pair of electrodes is placed on the other side of the fixed boundary as a sensor to acquire the vibration energy and convert voltage signals, as shown in Figure 2. The electrode size is determined by the force conservative equation, thus leading to two designs. First, when the input electrode is close to the fixed end and has a length of $4.4 \mathrm{~mm}$, the actuator can generate maximum power. Second, when the output electrode is close to the fixed end and has a shorter length, a larger open circuit output voltage is obtained. In our previous study, output voltages as high as twice the input voltages have been reported [29]. To obtain maximum power, we determine the length of the input and output electrodes to be $4.4 \mathrm{~mm}$. The width and thickness of the 
electrodes were $5 \mathrm{~mm}$ and $10 \mu \mathrm{m}$, respectively. The upper electrode is fabricated using screen-printed silver.

Figure 2. Top electrodes, PZT and bottom electrodes for piezoelectric vacuum sensors.

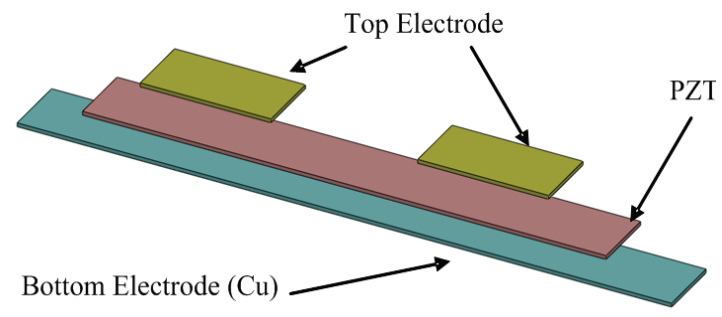

\subsection{Design and Production of Fixtures}

To allow the sensor to maintain the same boundary conditions during each measurement, we designed a fixture that can keep the cantilever beam fixed at both ends. The fixture contains two parts: upper cover and base. The upper cover has two holes for electrical wires. The clamped-clamped piezoelectric beam is placed in a trench in the base. Four M2 screws were used to fix the upper cover and the base which is shown in Figure 3. The fixture is made by transparent acrylic material which has advantages such as low density, high mechanical strength, good tensile and impact resistance, high transparency, low cost, and ease of machining.

Figure 3. Schematic diagram of fixtures.

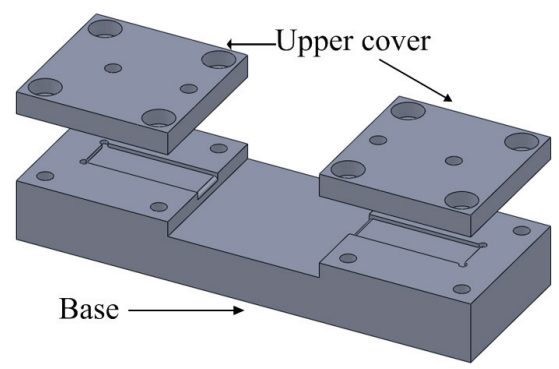

\section{Experimental Setup and Procedure}

\section{Laboratory Equipment and Experimental Setup}

The vacuum system consists of a stainless steel vacuum chamber, a mechanical pump, a turbo molecular pump, a gas flow controller and reference vacuum gauges. A mechanical pump (DOU 16B Balzers, Albuquerque, NM, USA) was first used to exhaust the gas at atmospheric pressure to achieve vacuum condition. When pressure was down to $10^{-2}$ Torr, a turbomolecular pump (Turbo VAC 450, Leybold, Cologne, Germany) was then used to obtain high vacuum $\left(10^{-2}\right.$ to $6.5 \times 10^{-6}$ Torr). However, it might take more than $24 \mathrm{~h}$ to achieve $1 \times 10^{-6}$ Torr using our pumping system. To reach certain pressure accurately, a gas flow controller was used to flow certain amount of 
nitrogen or argon into chamber. Two reference vacuum gauges, Pirani gauge and cold cathode gauge, were used. Pirani gauge is able to measure the pressure between 760 to $10^{-3}$ Torr and cold cathode gauge is capable to measure the pressure between $10^{-2}$ to $10^{-9}$ Torr.

The experimental setup is shown in Figure 4. The clamped-clamped piezoelectric pressure sensor was fixed by the fixture and was placed in the vacuum chamber. Electrical feedthroughs were used to transfer electrical signals through the vacuum system wall. A sine wave was generated by a function generator (33220A, Agilent, Santa Rosa, CA 95403-1738, USA) and amplified by a power amplifier (PZD700, TREK, Lockport, NY, USA) to excite the piezoelectric beam at first resonance frequency. At the mean time, a spectrum analyzer (Agilent 35670A) was used to measure the frequency response of input and output voltages.

Figure 4. Experimental setup.

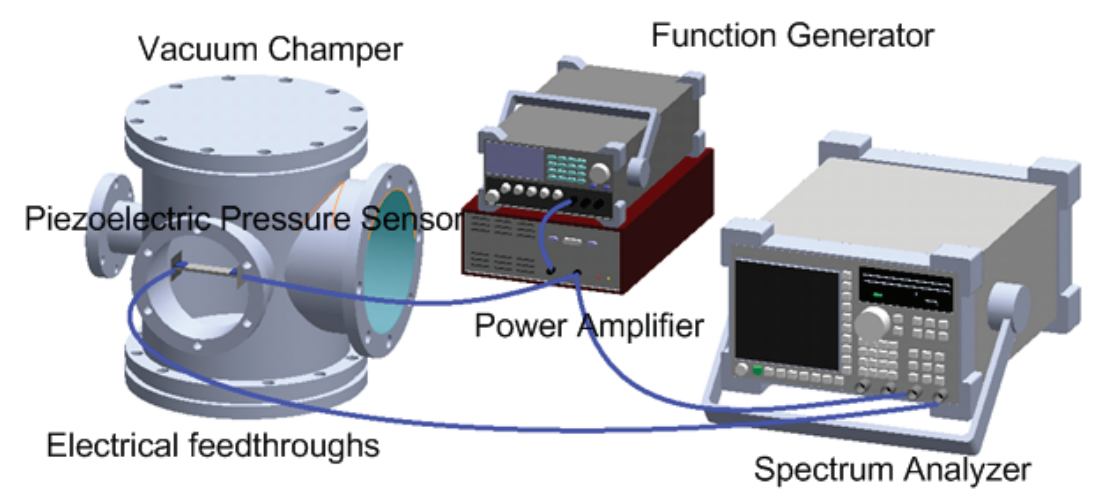

\section{Results and Discussion}

\subsection{Frequency Response under Different Pressures}

Frequency response functions were obtained by the following steps. The spectrum analyzer generated a swept-sine signal to drive a pair of electrodes through an amplifier. In the meantime, the other pair of electrodes generated electric output. Both the swept-sine signal and the output voltage were fed back to the spectrum analyzer to calculate the frequency response function. To maximum sensor output, the piezoelectric beams were excited at the first resonance frequencies under different pressures condition in the following experiments. The data from measured frequency response functions were processed to extract damping ratios using half power method. Figure 5 shows the frequency response functions of a clamped-clamped piezoelectric beam under different pressures when the residue gas in the vacuum chamber is nitrogen. The clamped-clamped piezoelectric beams have maximum output and input ratio when the beams were excited at resonance frequencies. The resonance frequencies of the piezoelectric beam under the vacuum pressures at $5 \times 10^{-6}, 7.5 \times 10^{-4}, 1$ and 75 Torr were $3100,3067,3038$ and $3030 \mathrm{~Hz}$, respectively. When the pressures in the vacuum chamber decreased, the resonance frequencies and the resonance amplitudes of beam decreased because the damping coefficient of gas increased. The difference of first resonance frequencies of the vacuum sensor between 75 torr and $5 \times 10^{-6}$ torr was just $2.25 \%$ 
$(70 \mathrm{~Hz})$ because of tiny damping variation. The resonance amplitude, output and input voltage ratio, were $0.0063,0.0059,0.0052$ and 0.0032 , respectively, when the pressures were $5 \times 10^{-6}$, $7.5 \times 10^{-4}, 1$ and 75 Torr.

Figure 5. Frequency response of sensing components.

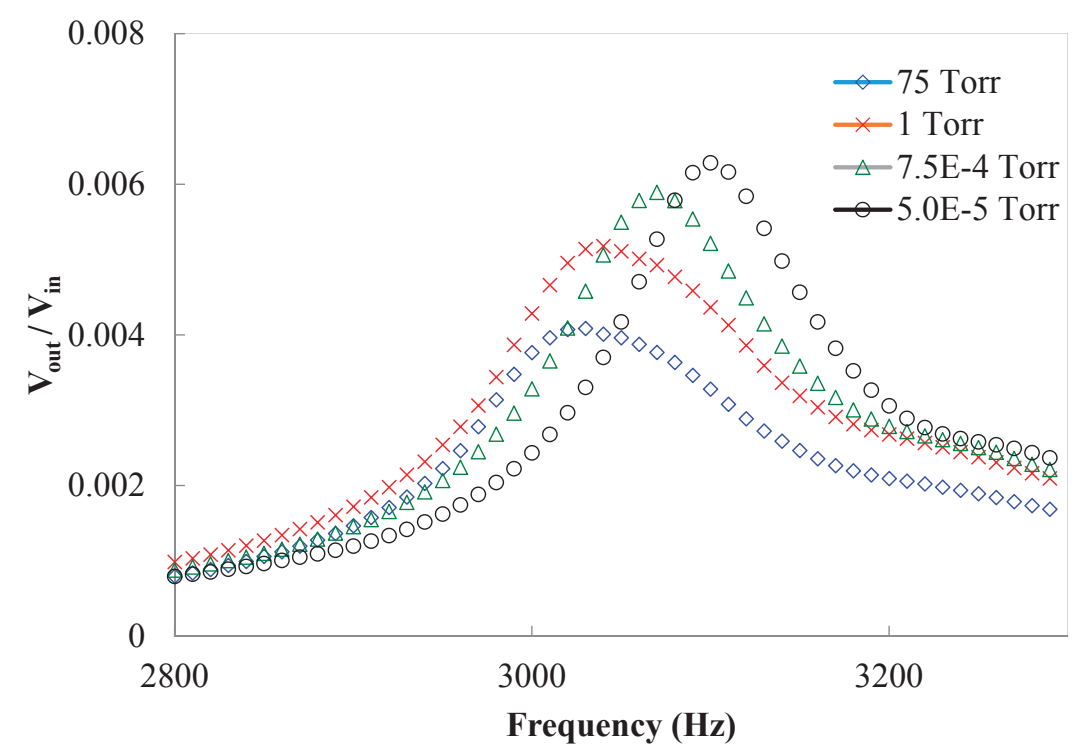

\subsection{Relationship between Pressure and Output Voltage}

Figure 6 showed the resonance amplitudes (Vout/Vin) versus the vacuum pressures in the chambers when the residue gas is nitrogen. Each operating frequency corresponding to the maximum amplitude values is applied in different vacuum pressure. In the experimental results, each pressure value corresponds to a piezoelectric output ratio. The vacuum pressure from $6.5 \times 10^{-6}$ to 760 Torr can be directly derived from piezoelectric output ratio. Note that each experiments corresponding to different pressures were repeated 10 times. Finally, the average values of output voltage were reported. The data have been plotted on a log-log plot to show the extreme range of both the measured maximum amplitude and imposed air pressure. All the experimental results appear to have the same general trend, showing decreasing damping values with decreasing pressure. Clearly these experimental results support both the rarefied and the viscous theories. The pressure in the vacuum region is divided into three ranges for further analysis. Vacuum pressure below $10^{-3}$ Torr belonging to molecular flow is known as high vacuum; vacuum pressure in the range $10^{-3}-1$ Torr belonging to transition flow is known as medium vacuum; vacuum pressure greater than 1 Torr belonging to viscous flow is known as low vacuum. We use the linear regression method to deal with the results of each segment to obtain the best linear data. After processing, each line segment will be discussed. Pandey et al. [29] reported a paper to discuss effect of pressure on fluid damping in MEMS torsional resonators with flow ranging from continuum to 
molecular regime. Their results also indicated that the quality factors of devices varied in different flow regions.

Figure 6. The resonance amplitudes (Vout/Vin) versus the vacuum pressures in the chambers when the residue gas is nitrogen.

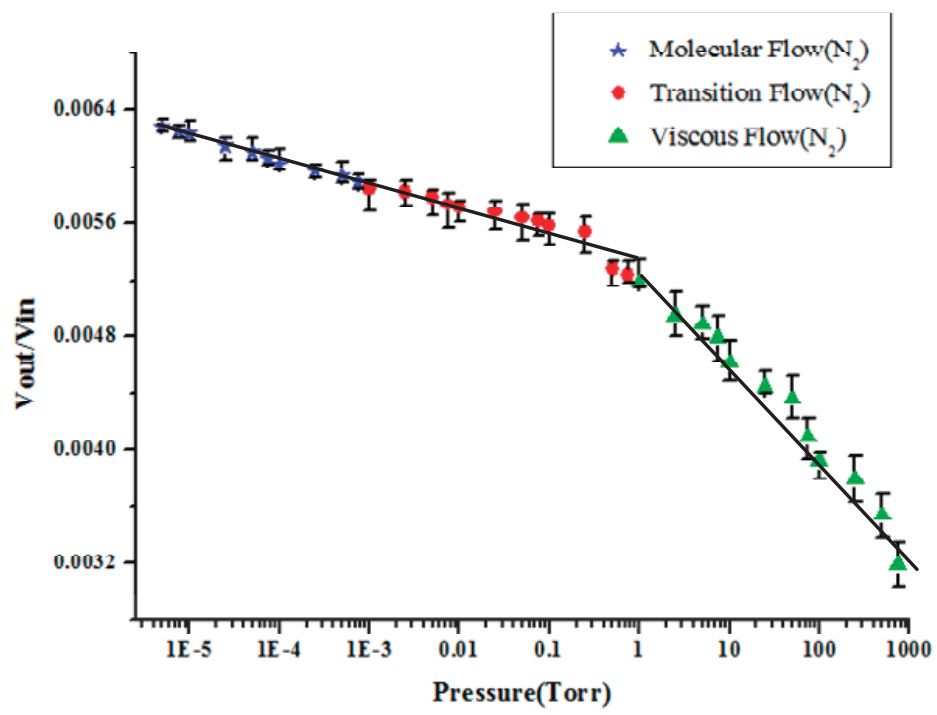

The slopes of the voltage $v s$. pressure in the viscous flow region is $\mathrm{y}=-0.0002 \mathrm{x}+0.0053$ and $\mathrm{R}^{2}=0.993$. The slopes of the voltage $v s$. pressure in the transition flow region is $\mathrm{y}=-0.0003 \mathrm{x}+$ 0.0052 and $\mathrm{R}^{2}=0.8913$. The slopes of the voltage $v s$. pressure in the molecular flow region is $\mathrm{y}=-0.0007 \mathrm{x}+0.0054$ and $\mathrm{R}^{2}=0.971$. The slope of the voltage $v s$. pressure curve in the viscous flow region is greater than that in the transition flow region, while the slope in the transition flow region is greater than that in the molecular flow region. From this result, we infer that the gas viscosity force of viscous flow is greater than the other two flows. Also, the force due to change in gas viscosity for viscous flow is more obvious than that for transition flow and molecular flow. Therefore, the change in the piezoelectric output ratio is the most obvious in viscous flow. However, fitting in three regions may not be the best solution. It also looked that the data in the molecular flow and transition flow region could be fitted with one straight line instead of two. The slopes of the voltage $v s$. pressure from $5 \times 10^{-6}$ to 1 torr is $\mathrm{y}=-0.00022 \mathrm{x}+0.0053$ and $\mathrm{R}^{2}=0.924$. The slopes of the voltage $v s$. pressure from 1 to 750 torr is $\mathrm{y}=-0.0007 \mathrm{x}+0.0054$ and $\mathrm{R}^{2}=0.971$.

\subsection{Relationship between Pressure and Damping Ratio}

The data from measured frequency response functions in the previous experiments were processed to extract damping ratios using half power method. After processing, we obtain the damping ratio corresponding to the respective pressure. Figure 7 showed that the damping ratios 
versus the vacuum pressures in the chamber when the residue gas is nitrogen. The slopes of the damping ratios $v s$. pressure in the viscous flow region is $\mathrm{y}=0.0009 \mathrm{x}+0.0206$ and $\mathrm{R}^{2}=0.957$. The slopes of the damping ratios $v s$. pressure in the transition flow region is $\mathrm{y}=0.0018 \mathrm{x}+0.0226$ and $\mathrm{R}^{2}=0.8574$. The slopes of the damping ratios $v s$. pressure in the molecular flow region is $\mathrm{y}=0.0042 \mathrm{x}+0.0225$ and $\mathrm{R}^{2}=0.9592$. However, fitting in three regions may not be the best solution. It also looked that the data in the molecular flow and transition flow region could be fitted with one straight line instead of two. The slopes of the voltage $v s$. pressure from $5 \times 10^{-6}$ to 1 torr is $\mathrm{y}=0.0013 \mathrm{x}+0.0216$ and $\mathrm{R}^{2}=0.916$.

Figure 7. The damping ratios versus the vacuum pressures in the chamber when the residue gas is nitrogen.

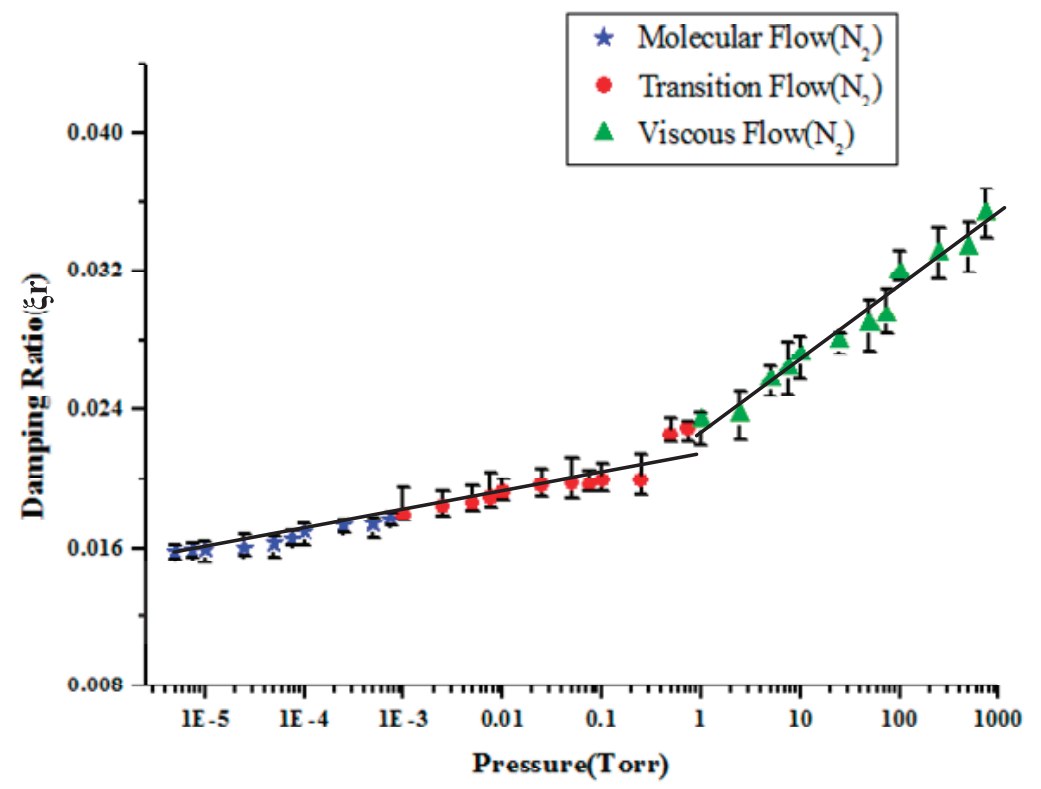

The measured damping ratio is the sum of structural damping and gas damping. Experimental results showed that the greater the quantity of residual gas in the vacuum chamber, the larger is the damping effect for the sensing element caused by the residual gas. Greater pressure indicates a larger number of gas molecules in the vacuum chamber. Therefore, there is a greater probability of collisions between gas molecules and piezoelectric beam; this situation increases the gas damping effect for the sensing element when the pressure increases. When the residual gas in the vacuum chamber was rarer, small damping effects for the sensing element were obtained experimentally.

\subsection{Different Residue Gases-Nitrogen and Argon}

Figure 8 shows the resonance amplitudes (Vout/Vin) versus the vacuum pressures in the chambers when the residue gases are nitrogen and argon. However, the resonance amplitudes of the sensing element in the argon were just performed in the vacuum pressure from $6.5 \times 10^{-6}$ to 1 Torr 
due to the limitation of the gas flow controller. We find that the output result has similar trends for both nitrogen and argon in the vacuum pressure from $6.5 \times 10^{-6}$ to 1 Torr. The output value changes marginally. Under the same pressure, the output value of nitrogen is larger than that of argon. We can conclude that the vibration of the cantilever beam affected by the viscous force caused by argon is larger than that caused by nitrogen. The mass of argon (39.948 amu) is larger than that of nitrogen $(28 \mathrm{amu})$. The resistance force of the molecular collision of argon is larger than that of nitrogen.

Figure 8. The resonance amplitudes (Vout/Vin) versus the vacuum pressures in the chambers when the residue gases are nitrogen and argon.

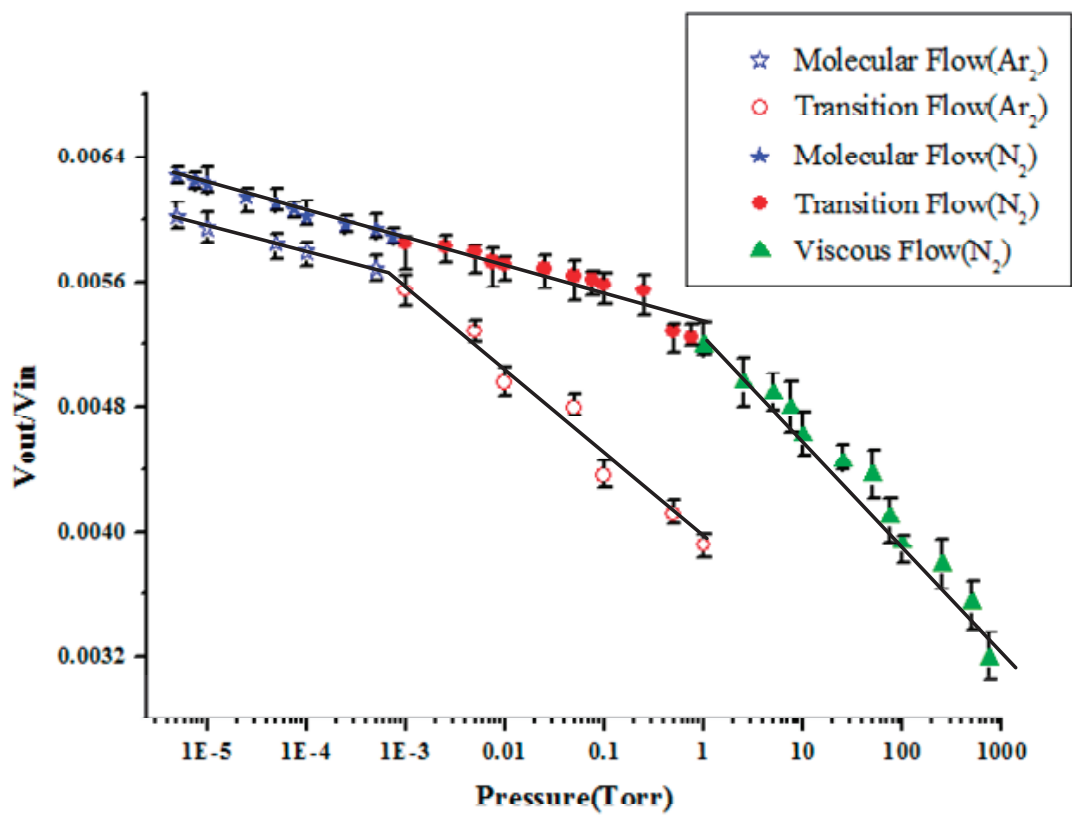

Each experimental corresponding to different pressures under Nitrogen and Argon was repeated 10 times and the average values of output voltage were reported. Experimental results were consistent if that boundary condition remained the same. Pressure measurement was taken after observing the steady state condition to prevent measurement errors. However, the duration of steady state condition varies for the flow in the continuous region to the flow in the molecular region. One of the samples was leaved in the vacuum chamber for 3 months and there were no significant difference even the sample was driven for a long time. However, there still need further research to study the consistency and stability of the system. There also still need further research to study the different gases to verify the consistency and stability of the system. 


\section{Conclusions}

In this study, we developed a clamped-clamped beam-type piezoelectric vacuum pressure sensor. The sensor was designed using two piezoelectric elements: for actuating and sensing. Applying voltage to the PZT actuating element causes deformation of the cantilever. At the other end of the beam, the PZT sensing element produces a voltage caused by the bending of the beam. The piezoelectric pressure sensing element was developed that can be used to measure a wide range of vacuum pressure from $5 \times 10^{-6}$ to 760 Torr. From low to high vacuum, the output and input voltage ratio (Vout/Vin) gradually increased with decrease in pressure. The relationship between vacuum pressure and damping ratio was obtained for pressure from $5 \times 10^{-6}$ to 760 Torr. In high vacuum, the damping ratio is less than that in low vacuum. Finally, the sensing elements were used to measure the pressure in nitrogen and argon to study the relationship between vacuum pressures and damping ratios of different gases. Comparison of the output voltage ratios in argon and nitrogen showed that the damping ratios follow the same trend as the vacuum pressure. The damping ratio of argon is greater than that of nitrogen because the mass of argon is larger.

\section{Acknowledgments}

The authors would like to thank the Ministry of Science and Technology of Taiwan for financial support of this research under grant numbers 102-2221-E-005-018 and 102-2221-E-492-015.

\section{Author Contributions}

Bing-Yu Wang, Fan-Chun Hsieh, Fong-Zhi Chen and Chia-Che Wu developed the original direction and methodology behind the work; Bing-Yu Wang, Che-Yu Lin, Shao-En Chen and Chia-Che $\mathrm{Wu}$ conceived and designed the experiments; Fan-Chun Hsieh, Che-Yu Lin and Shao-En Chen performed the experiments; Bing-Yu Wang and Chia-Che Wu analyzed the data and prepared the manuscript. All authors reviewed the manuscript before submission and approved its publication.

\section{Conflicts of Interest}

The authors declare no conflict of interest.

\section{References}

1. Kim, J.; Novick, B.J.; Desimone, J.M.; Carbonel, R.G. Ultrathin Film Deposition by Liquid $\mathrm{CO}_{2}$ Free Meniscus Coatings Uniformity and Morphology. J. Langmuir. 2006, 22, 642-657.

2. De Araújo, F.O.; de Almeida, E.O.; Alves, C., Jr.; da Costa, J.A.P.; Dumelow. T. Deposition of $\mathrm{TiO}_{2}$ on silicon by sputtering in hollow cathode J. Surface Coat. Technol. 2006, 201, 2990-2993.

3. Buder, U.; von Klitzing, J.-P.; Obermeier, E. Reactive ion etching for bulk structuring of polyimide. Sens. Actuators A Phys. 2006, 132, 393-399. 
4. Bu, I.Y.Y.; Flewitt, A.J.; Miline, W.I. Nanocrystalline Silicon Thin Films Fabricated at $80{ }^{\circ} \mathrm{C}$ by Using Electron Cyclotron Resonance Chemical Vapor Deposition. J. Plasma Sci. Technol. 2010, 12, 608-613.

5. Watanabe, Y. Formation and behaviour ofnano/micro-particles in lowpressure plasmas. J. Phys. D: Appl. Phys. 2006, 39, R329-R361.

6. Vankova, N.; Tcholakova, S.; Denkov, N.D.; Ivanov, I.B.; Vulchev, V.D.; Danner, T. Emulsification in turbulent flow 1. Mean and maximum drop diameters in inertial and viscous regimes. J. Colloid Interface Sci. 2007, 312, 363-380.

7. Lodefier, K.; Dick, E. Modelling of Unsteady Transition in Low-Pressure Turbine Blade Flows with Two Dynamic Intermittency Equations. J. Flow Turbul. Combust. 2005, 76, 103-132.

8. Khakoo, M.A.; Blumer, J.; Keane, K.; Campbell, C.; Silva, H.; Lopes, M.C.A.; Winstead, C.; McKoy, V.; da Costa, R.F.; Ferreira, L.G.; et al. Low-energy electron scattering from methanol and ethanol. J. Phys. Rev. A 2008, 77, 042705.

9. Zhang, F.T.; Tang, Z.; Yu, J.; Jin, R.C. A micro-Pirani vacuum gauge based on micro-hotplate technology. Sens. Actuators A Phys. 2006, 126, 300-305.

10. Brun, T.; Mercier, D.; Koumela, A.; Marcoux, C.; Duraffourg, L. Silicon nanowire based Pirani sensor for vacuum measurements. J. Appl. Phys. Lett. 2012, 101, 183506.

11. Jay Mitchell, G.; Lahiji, R.; Najafi, K. An Improved Performance Poly-Si Pirani Vacuum Gauge Using Heat-Distributing Structural Supports. J. Microelectromechanical Syst. 2008, 17, 93-102.

12. Robinson, A.M.; Haswell, P.; Lawson, R.P.W. A thermal conductivity microstructural pressure sensor fabricated in standard complementary metal-oxide semiconductor. Rev. Sci. Instrum. 1992, 63, 2026-2029.

13. Singh, K.J.; Elmazria, O.; Sarry, F.; Nicolay, P.; Ghoumid, K.; Belgacem, B.; Mercier, D.; Bounouar, J. Enhanced Sensitivity of SAW-Based Pirani Vacuum Pressure Sensor. IEEE Sens. J. 2011, 11, 1458-1464.

14. Weng, P.K.; Shie, J.-S. Micro-Pirani vacuum gauge. Rev. Sci. Instrum. 1994, 65, 492-499.

15. Yang, Y.C.; Qian, L.; Tang, J.; Liu, L.; Fan, S.S. A low-vacuum ionization gauge with HfC-modified carbon nanotube field emitters. Appl. Phys. Lett. 2008, 92, 153105.

16. Choi, I.-M.; Woo, S.-Y. Application of carbon nanotube field emission effect to an ionization gauge. Appl. Phys. Lett. 2005, 87, 173104.

17. Choi, I.-M.; Woo, S.-Y.; Song, H.-W. Improved metrological characteristics of a carbon-nanotube-basedionization gauge. J. Appl. Phys. Lett. 2007, 90, 023107.

18. Hogg, J.L. Friction and Force Due to Transpiration as Dependent on Pressure in Gases. Proc. Am. Acad. Arts Sci. 1906, 42, 115-146.

19. Kobayashi, Y.; Kurokawa, A. Quartz friction gauge for monitoring the concentration and viscosity of Natural Hy mixtures. Vac. Sci. Technol. A 2007, 25, 1246-1250.

20. Tenholte, D.; Kurth, S.; Geßner, T.; Dotzel, W.D. A MEMS friction vacuum gauge suitable for high temperature environment. Sens. Actuators A Phys. 2008, 142, 166-172.

21. Park, C.-S.; Lee, D.-W. A micromachined pressure sensor based on an array of microswitches. Rev. Sci. Instrum. 2010, 81, 055103. 
22. Zheng, X.J.; Cao, X.C.; Sun, J.; Yuan, B; Li, Q.H.; Zhu, Z.; Zhang, Y. A vacuum pressure sensor based on $\mathrm{ZnO}$ nanobelt film. Nanotechnology 2011, 22, 435501.

23. Gronych, T.; Ullman, R.; Peksa, L.; Repa, P. Measurements of the relative momentum accommodation coefficient for different gases with a viscosity vacuum gauge. Vacuum 2004, 73, 275-279.

24. Fremerey, J.K. The Spinning Rotor Gauges. Vac. Sct. Tachnol. A 1985, 3, 1715-1720.

25. Sumali, H.; Meissner, K.; Cudney, H.H. A piezoelectric array for sensing vibration modal corrdinates. Sens. Actuators A Phys. 2001, 93, 123-131.

26. Mortet, V.; Petersen, R.; Haenen, K.; D’Olieslaeger, M. Wide range pressure sensor based on a piezoelectric bimorph microcantilever. J. Appl. Phys. Lett. 2006, 88, 133511.

27. Wang, Y.-T.; Hu, T.-C.; Tong, C.-J.; Lin, M.-T. Novel full range vacuum pressure sensing technique using free decay of trapezoid micro-cantilever beam deflected by electrostatic force. Microsyst. Technol. 2012, 18, 1903-1908.

28. Wu, C.-C.; Chen, C.-S. An electromechanical model for a clamped-clamped beam type piezoelectric transformer. Microsyst. Technol. 2012, 18, 1771-1778.

29. Pandey, A.K.; Pratap, R.; Chau, F.S. Effect of pressure on fluid damping in MEMS torsional resonators with flow ranging from continuum to molecular regime. Exp. Mech. 2008, 48, 91-106. 


\section{MDPI AG}

Klybeckstrasse 64

4057 Basel, Switzerland

Tel. +41616837734

Fax +41613028918

http://www.mdpi.com/

Sensors Editorial Office

E-mail: sensors@mdpi.com

http://www.mdpi.com/journal/sensors 



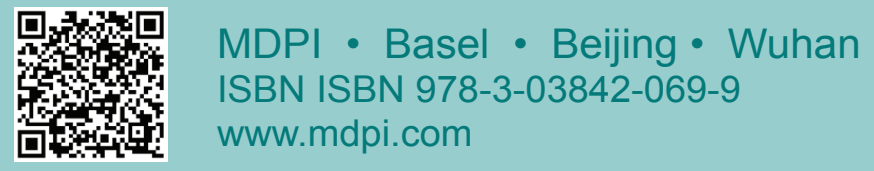

